\title{
Bioactive Compounds from Marine Sources
}

\author{
Dem Fachbereich Maschinenbau und Verfahrenstechnik \\ der Technischen Universität Kaiserslautern \\ zur Erlangung des akademischen Grades
}

\author{
Doktor-Ingenieur (Dr.-Ing.) \\ vorgelegte \\ Dissertation
}

\author{
von \\ Herrn \\ M. Sc. Ahmed Elsayed Ahmed Farag Zayed \\ aus Tanta, Ägypten
}

Kaiserslautern, 2018

Vorsitzender: Prof. Dr. Eberhard Kerscher

1. Referent: Prof. Dr. Roland Ulber

2. Referent: Prof. Dr. Nicole Frankenberg-Dinkel

mündliche Prüfung (Disputation) am: 26.06.2018

[D386] 
Printed with the support of the German Academic Exchange Service 


\section{$\underline{\text { Declaration }}$}

I hereby confirm that the dissertation was performed by myself and all used aids are specified in the work. In addition, either the dissertation or parts of it have not been submitted yet as examination papers for a state or other scientific institutes. Dissertation results have not been submitted also yet to another department or university.

\section{Date}

Signature

\section{Erklärung}

Hiermit bestätige ich, dass die Dissertation von mir selbst durchgeführt wurde und alle verwendeten Hilfsmittel in der Arbeit angegeben sind. Darüber hinaus sind die Dissertation oder Teile davon noch nicht als Prüfungsunterlagen für einen Staat oder andere wissenschaftliche Institute eingereicht worden. Dissertationsergebnisse wurden auch noch nicht an eine andere Abteilung oder Universität übermittelt.

\section{Datum}

Unterschrift 


\section{Acknowledgment}

It is my pleasure to thank all people whose thoughts, ideas, suggestions, reassurance, and support shaped this piece of research. First, I would like to thank ALMIGHTY ALLAH (MY GOD) for continuously blessing me with persistence, health, and faith to complete my $\mathrm{Ph}$. D. Degree.

All gratitude must be extended to my supervisor Prof. Dr. rer. nat. Roland Ulber for giving me the opportunity to perform my thesis work independently at the Institute of Bioprocess Engineering, TU Kaiserslautern. It was always possible to ask questions and to get encouragement and precious advice to go on with my work. Especially in hard times his support was of great value.

Special thanks and appreciation must be for Prof. Dr. Kai Muffler, who was my previous group leader. Useful discussions and information exchange were very helpful even after he left our institute.

I would like to express my appreciation and gratitude to Prof. Dr. Nicole Frankenberg-Dinkel and co-workers for co-operation in molecular biology work.

In addition, I would like to extend my appreciation to Prof. Dr. Steffen Rupp, Dr. Thomas Hahn Mrs. Doris Finkelmeier, and Dr. Anke Burger-Kentischer from Fraunhofer Institute for Interfacial Engineering and Biotechnology (IGB) for performing anti-viral, anti-fungal, antibacterial and cytotoxic studies.

Prof. Dr. Roland Krämer from Heidelberg University is also grateful for his donation of perylene diimide derivative compound who synthesized it personally in his lab.

I would also like to acknowledge the cooperation and creativity of Dr. med. Michael Püttmann and Mrs. Gabi Bruckmann for their help in anticoagulant measurements, Dr. Broder Rühmann and Dr. Jochen Schmid for compositional analysis and Prof. Dr. Lothar Elling for performing the MP-CE analysis.

Thanks are due to my colleagues at the institute for interesting discussions making them more than colleagues but friends. They have challenged me to "grow into" an interesting and challenging research culture.

The Project was funded by the Egyptian Ministry of Higher Education and DAAD (Funding program number 57076387), so both are greatfully acknowledgment and the fruitful co.operation between Egypt and Germany.

Finally, I extend my deepest gratitude to all the members of my family for their continuing prayers, encouragement, understanding, and support. They have guided and encouraged me with great energy and endless faith in my abilities. They never doubted I would really finish my dissertation one day. My children, Mariam and Mooaz, suffered my anxieties, bad moods and absences (both physical and mental). I am truly grateful for my wife Aya Abdella for her constant assistance, encouragement, and understanding and highly appreciated backup.

\footnotetext{
Ahmed Zayed

Kaiserslautern, 29.06.2018
} 


\section{List of Abbreviations}

- ${ }^{1} \mathrm{H}-\mathrm{NMR}$

- 2,4-D

- AHT

- APPT

- $\quad$ ASP-12 NTA

- BAP

- BSA

- $\mathrm{CHNS}$

- CMV

- CPPU

- DEAE-C

- DMF

- DMSO

- DPPH

- E

- EAE

- FAO

- FDA

- FPLC

- $\quad$ FT-IR

- FucTs

- GlucNAc

- GMP

- GPC/SEC

- GRAS

- GT

- $h$

- HEPES

- HMWF

- HPLC

- HSV

- i.d.
Proton-Nuclear Magnetic Resonance spectroscopy

2,4-Dichlorophenoxyacetic acid

Anhdrotetracyclin

Activated Partial Thromboplastin Time

Artificial Sea water Provasoli-12-Nitrilotriacetic acid

6-Benzylaminopurine

Bovine Serum Albumin

Carbon-Hydrogen-Nitrogen-Sulphur

Cyto Megalo Virus

1-(2-chloro-4-pyridyl)-3-phenylurea

Diethylaminoethyl-cellulose

Dimethyl Formamide

Dimethyl Sulfoxide

2,2-Diphenyl-1-picrylhydrazyl radical

Einstein

Enzyme-aided Extraction

Food and Agriculture Organization

Food and Drug Administration

Fast Protein Liquid Chromatography

Fourier Transform Infrared spectroscopy

Fucosyltransferase

$N$-Acetyl Glucosamine

Good Manufacturing Practice

Gel Permeation Chromatography/ Size Exclusion Chromatography

Generally Recognized As Safe

Glycosyltransferase

hour

4-(2-hydroxyethyl)-1-piperazineethanesulfonic acid

High Molecular Weight Fucoidan

High Performance/Pressure Liquid Chromatography

Herpes Simplex Virus

Internal diameter 
- IAA

- $\mathrm{IC}_{50}$

- IEX

- ISI

- $\mathrm{K}$

- $\mathrm{kDa}$

- L:D

- LMWF

- $\mathrm{m}$

- $\mathrm{MAB}$

- MAE

- $\quad$ MES

- $\min$

- $\mathrm{Mn}$

- $\quad \mathrm{Mp}$

- Mw

- MWCO

- OD

- PABA

- PAP

- PAPA

- PAPS

- PBS

- PCR

- PDD

- PDI

- PES

- PESA

- PMP

- psi

- PT

- PTC

- RFU
Indole-3-acetic acid

Half maximal Inhibitory Concentration

Ion Exchange Chromatography

Institute for Scientific Information

6-Furfurylaminopurine (Kinetin)

Kilo Dalton

Light: Dark light regime

Low Molecular Weight Fucoidan

Meter

Maleic Acid Buffer

Microwave aided or assisted extraction

2-(N-morpholino)ethanesulfonic acid

Minute

Number-Average Molecular Weight

Peak Molecular Weight

Average-Weight Molecular Weight

Molecular Weight Cut-Off

Optical Density or Absorbance

para-amino benzoic acid

3`-phosphoadenosine 5'-phosphate

para-amino phthalic acid

3'-phosphoadenosine 5'-phospho-sulphate

Phosphate-buffered Saline

Polymerase Chain Reaction

Perylene Diimide Derivative

Polydispersity Index

Provasoli Enriched Sea water

Provasoli Enriched Sea water Alternative (without vitamins)

1-phenyl-3-methyl-5-pyrazolone

Pounds per Square Inch

Prothrombin Time

Plant Tissue Culture

Relative Fluorescence Unit 


$\begin{array}{ll}- & \text { RI } \\ \text { - } & \text { rpm } \\ \text { - } & \text { S } \\ \text { - } & \text { S } \\ \text { - } & \text { SAR } \\ \text { - } & \text { SDS } \\ \text { - } & \text { SDS-PAGE } \\ \text { - } & \text { Sepabeads }{ }^{\circledR} \text { EC-EA } \\ \text { - } & \text { STC } \\ \text { - } & \text { STs } \\ \text { - } & \text { TA } \\ \text { - } & \text { TB } \\ \text { - } & \text { TPF } \\ \text { - } & \text { Tris } \\ \text { - } & \text { TT } \\ \text { - } & \text { TTC } \\ \text { - } & \text { UAE } \\ \text { - } & \text { UV/Vis } \\ \text { - } & \text { WHO }\end{array}$

- $\quad$ RI

Refractive Index

Rate per Minute

Siemens

second

Structure Activity Relationship

Sodium dodecyl Sulphate

Sodium Dodecyl Sulphate-PolyAcrylamide Gel Electrophoresis

Sepabeads ${ }^{\circledR}$ Enzyme carrier-Ethyl amino derivatized beads

Seaweed Tissue Culture

Carbohydrate Sulphotransferase

Thionin Acetate

Toluidine Blue

1,3,5-Triphenylformazan

tris(hydroxymethyl) aminomethane

Thrombin Time

2,3,5-Triphenyltetrazolium chloride

Ultrasound aided or assisted extraction

Ultraviolet-visible spectroscopy

World Health Organization

\section{Latin Symbols}

$-\lambda$

$-\quad \sum$

$-\delta$
- Wave length

- Sum

- Chemical shift 


\begin{abstract}
Fucoidan is a class of biopolymers mainly found in brown seaweeds. Due to its diverse medical importance, homogenous supply as well as a GMP-compliant product is of a special interest. Therefore, in addition to optimization of its extraction and purification from classical resources, other techniques were tried (e.g., marine tissue culture and heterologous expression of enzymes involved in its biosynthesis). Results showed that $17.5 \%(\mathrm{w} / \mathrm{w})$ crude fucoidan after pre-treatment and extraction was obtained from the brown macroalgae $F$. vesiculosus. Purification by affinity chromatography improved purity relative to the commercial purified product. Furthermore, biological investigations revealed improved anti-coagulant and anti-viral activities compared with crude fucoidan. Furthermore, callus-like and protoplast cultures as well as bioreactor cultivation were developed from $F$. vesiculosus representing a new horizon to produce fucoidan biotechnologically. Moreover, heterologous expression of several enzymes involved in its biosynthesis by E. coli (e.g., FucTs and STs) demonstrated the possibility to obtain active enzymes that could be utilized in enzymatic in vitro synthesis of fucoidan. All these competitive techniques could provide the global demands from fucoidan.
\end{abstract}

\title{
Zusammenfassung
}

Fuciodane sind Biopolymere mit sulfatiertem Homo- oder Heteropolysaccharidrückgrat. Sie kommen hauptsächlich in Braunalgen (Phaeophyta) vor. Durch ihre medizinische Bedeutung ist eine einheitliche Versorgung und GMP-konforme Produktion von besonderem Interesse. Aus diesem Grund wurden in dieser Arbeit verschiedene Techniken zur Fucoidan-Produktion angewendet (z.B. Optimierung von Extraktion und Aufreinigung aus klassischen Quellen, marine Gewebekultur und heterologe Expression von Enzymen, die in die Biosynthese von Fucoidan involviert sind). Die Ergebnisse zeigen, dass nach Vorbehandlung und Extraktion 17,5\% w/w Fucoidan aus der Braunalge $F$. vesiculosus gewonnen werden konnte. Durch Aufreinigung mittels Affinitätschromatographie konnte eine höhere Reinheit im Vergleich zu einem kommerziellen Produkt erreicht werden. Biologische Analysen ergaben verbesserte antikoagulante und antivirale Eigenschaften. Kallus- und Protoplastkulturen von F. vesiculosus, sowie Kulturen im Bioreaktor repräsentieren neue Wege, Fucoidan biotechnologisch herzustellen. Außerdem demonstrierte die heterologe Expression von mehreren Enzymen der Fucoidan-Biosynthese in E. coli (z.B. FucTs und STs) die Möglichkeit, aktive Enzyme zu erhalten, die in enzymatischer de-novo-Synthese von Fucoidan eingesetzt werden können. All diese Entwicklungen könnten dabei helfen, die globale Nachfrage an Fucoidan zu befriedigen. 


\section{Table of contents}

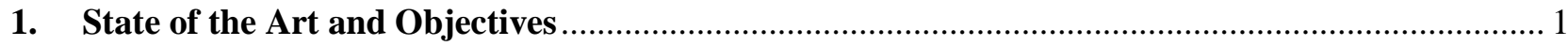

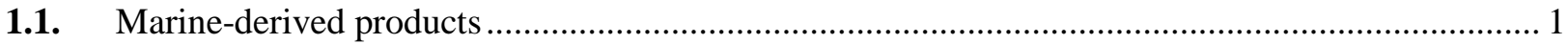

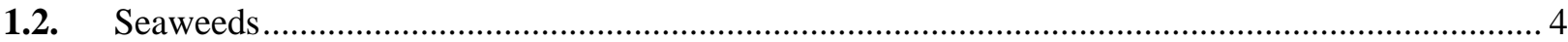

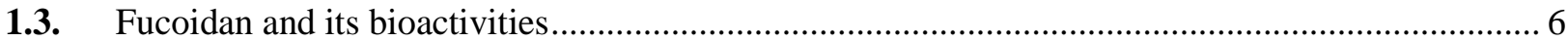

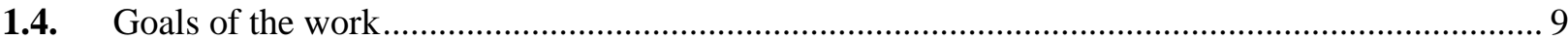

2. Optimization of Fucoidan Extraction and Purification from Brown Macroalgae ........................ 11

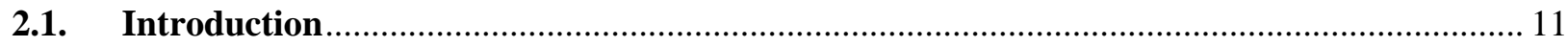

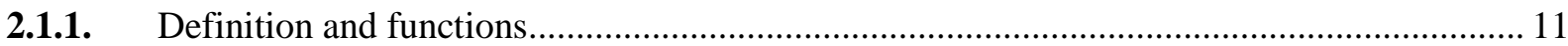

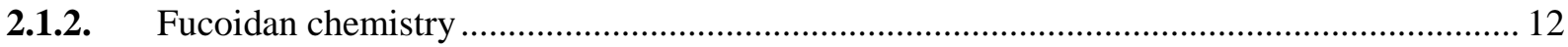

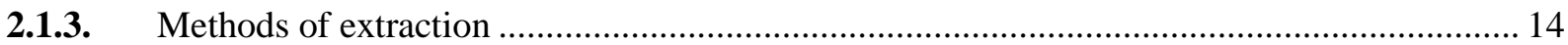

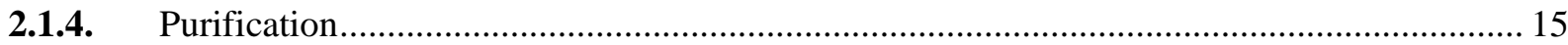

2.1.5. Fucoidan from $F$. vesiculosus or bladder wrack ............................................................ 17

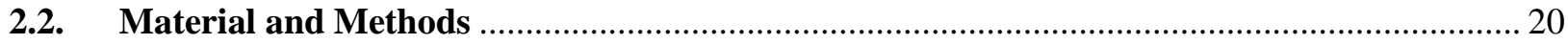

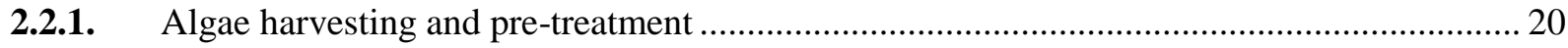

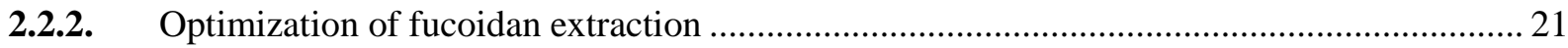

2.2.3. Quantitative assay for crude fucoidan fractions' contents ................................................. 23

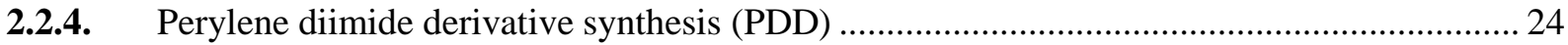

2.2.5. Immobilization of thiazine dyes and perylene diimide derivative ...................................... 24

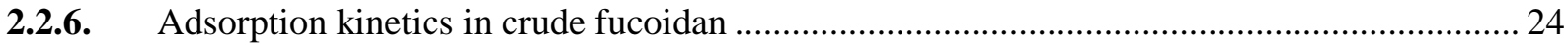

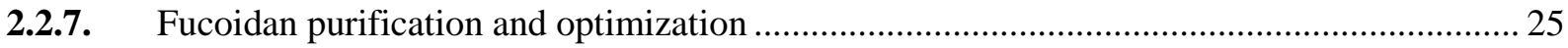

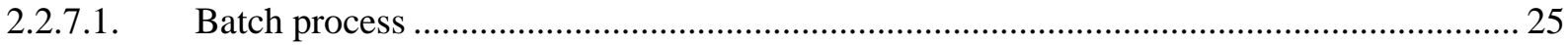

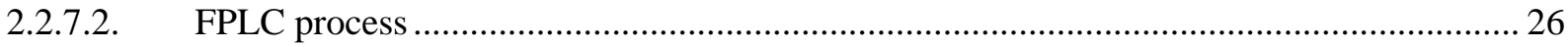

2.2.8. Purified fucoidan fractions characterization ..................................................................... 27

2.2.9. Process scaling-up and application of optimized conditions ….......................................... 31

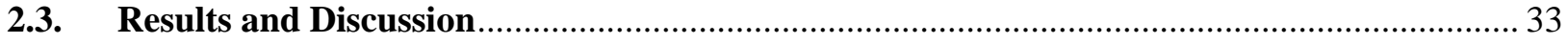

2.3.1. Pre-treatment, extraction and preparation of crude extract ................................................ 33

2.3.2. Characterization of crude fucoidan fractions ...................................................................... 34

2.3.3. Application and optimization of fucoidan purification ...................................................... 36

2.3.4. Application of PDD for fucoidan purification ................................................................. 44

2.3.4.1. Adsorption kinetic in crude fucoidan ........................................................................... 45

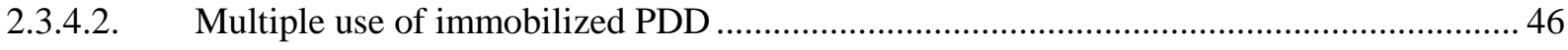

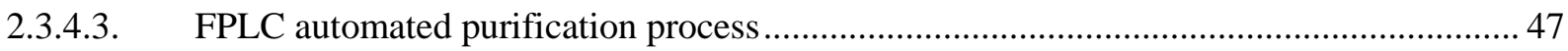

2.3.5. Physico-chemical characterization of purified fucoidan fractions ....................................... 48

2.3.5.1. Elemental Analysis (CHNS Analysis) ........................................................................... 48 


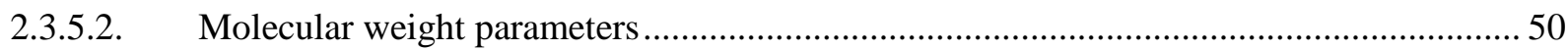

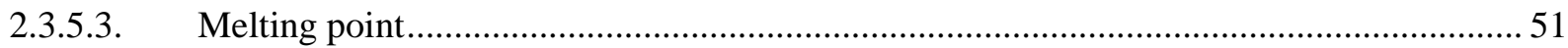

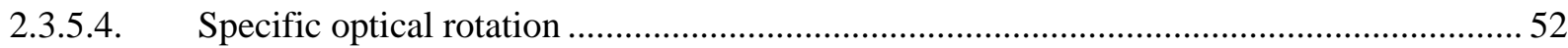

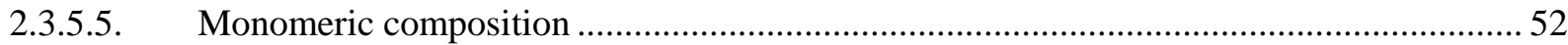

2.3.6. Spectroscopical identification of purified fucoidan fractions .............................................. 53

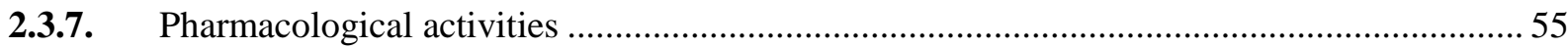

2.3.8. Process scaling-up and application of optimized conditions ….......................................... 62

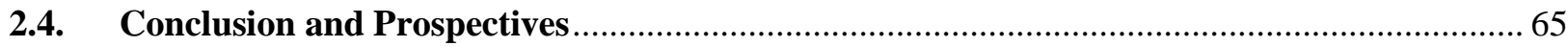

3. Development of Axenic Protoplast and Callus-like Cultures from F vesiculosus ........................ 66

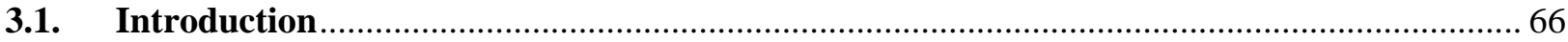

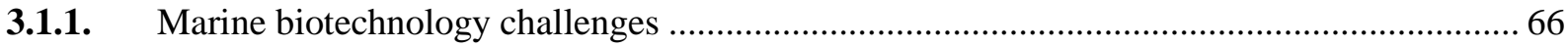

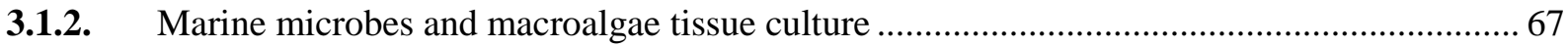

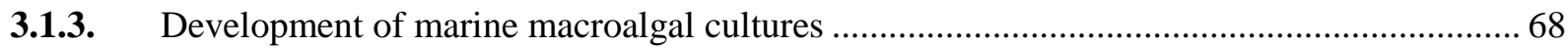

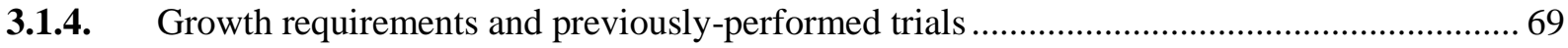

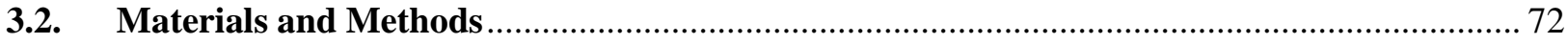

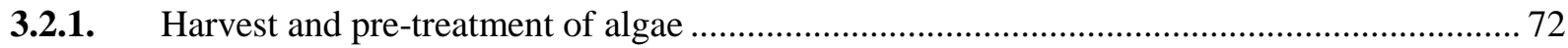

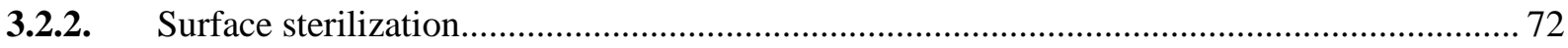

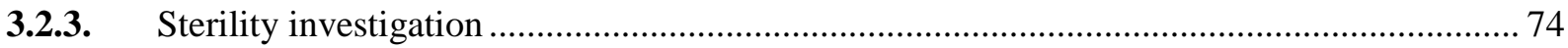

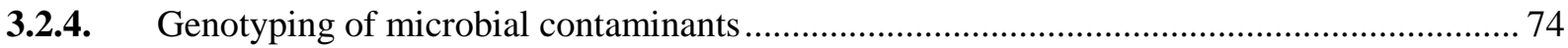

3.2.5. Vitality investigation (2,3,5-Triphenyltetrazolium chloride (TTC) assay) .......................... 75

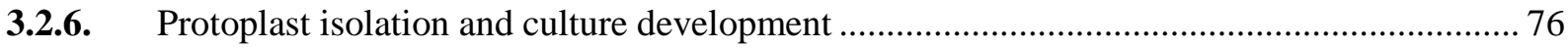

3.2.7. Development of protoplast and callus-like cultures ....................................................... 76

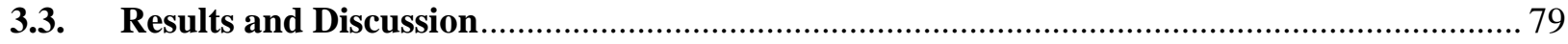

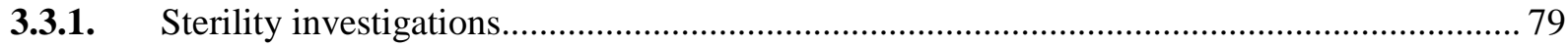

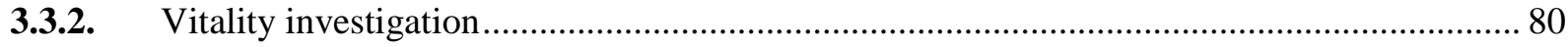

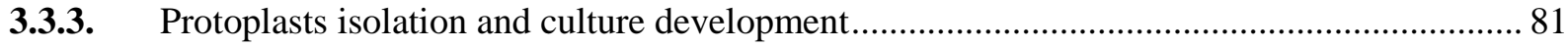

3.3.4. Callus-like development from $F$. vesiculosus explant ..................................................... 82

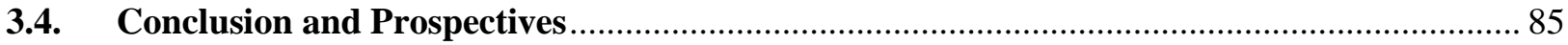

4. Heterologous Expression of Enzymes Involved in Fucoidan Biosynthesis .................................. 88

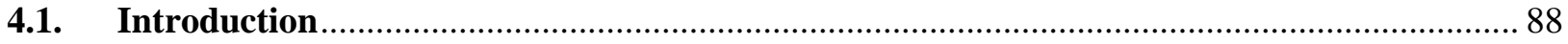

4.2. Heterologous expression of algal fucosyltransferases (FucTs) ......................................... 93

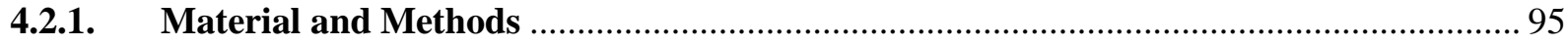

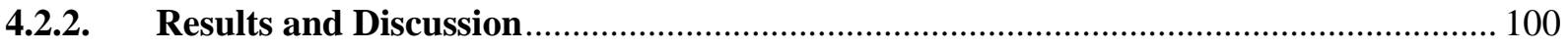

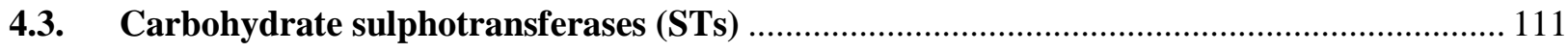

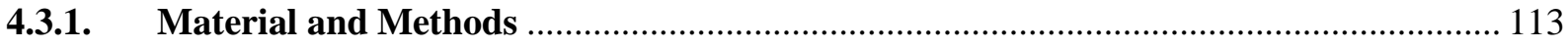




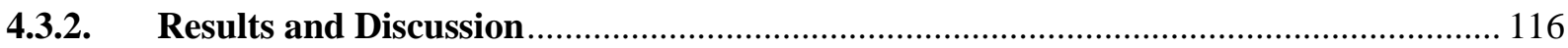

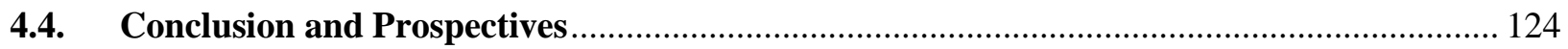

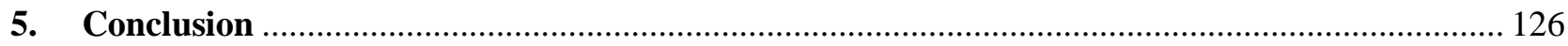

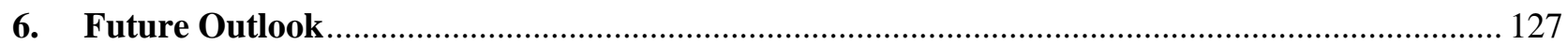

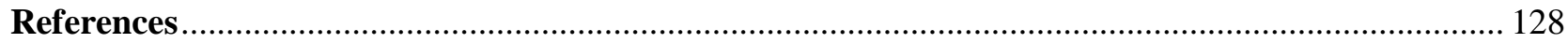

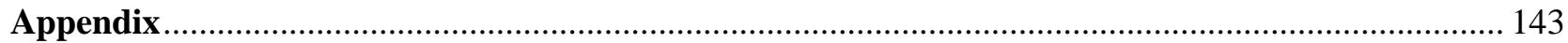

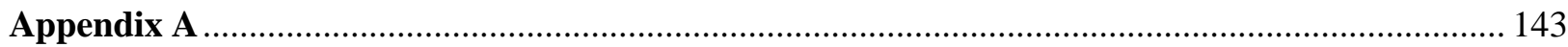

Chemical structures of some marine-derived bioactive compounds................................................. 143

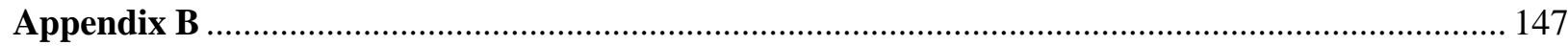

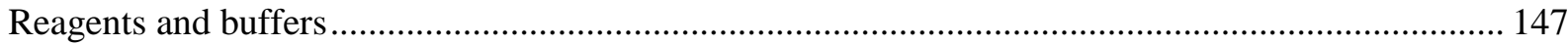

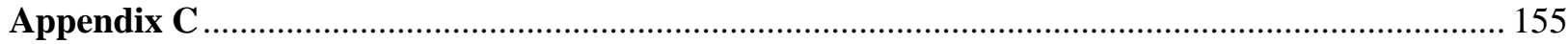

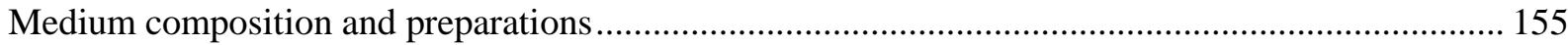

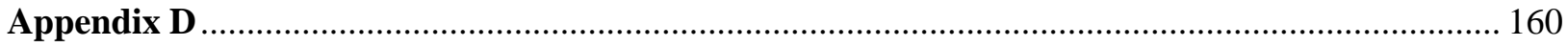

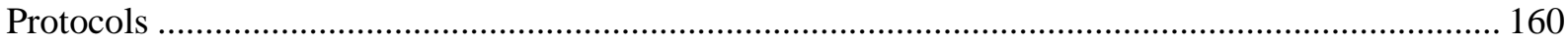

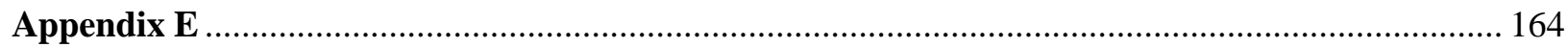

Calibration curves, instruments' charts and analysis reports ......................................................... 164

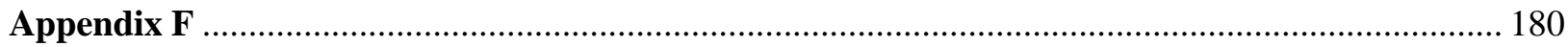

Lists of Figures, Tables, Schemes and devices ............................................................................ 180 



\section{State of the Art and Objectives}

Since the early life of the humankind on the Earth, nature represents the most powerful source for his major needs from food, energy and therapeutics. Oceans cover more than 70\% of the Earth's surface, and therefore, they continue to offer exceptional scaffolds improving the quality of the human life. For example, marine microbes, which represent $98 \%$ of ocean biomass [1], perform vital functions in global food chain cycle, $\mathrm{CO}_{2}$ fixation as well as assimilation of nutrients to maintain oxygen, nitrogen and phosphorous in their normal environmental levels [2].

In addition, the interest in marine-related fields has grown in the last period (e.g., taxonomy, ecology, chemistry,...etc.) to explore such source for bio- and chemical diversity and other valuables, such as the marine-derived bioactive compounds. The obtained results were promising and have encouraged the performance of further studies to discover new medicaments from natural sources and provide the global market with its required demands.

\subsection{Marine-derived products}

Marine organisms represent around 50\% of the worldwide biodiversity [3] in addition to their chemical and genetic diversities, and therefore represent a potential source of broad spectrums of commercially-valuable and diverse products, such as polysaccharides, enzymes, peptides, lipids, steroids and terpenoids [4,5]. From more than 300,000 described organisms, 12,000 novel compounds have been discovered attracting a great interest in the last decades [6]. Most of these compounds are produced as secondary metabolites as a defense mechanism to protect themselves against invaders. Marine-derived products have a wide range of applications in pharmaceuticals as anti-tumor and anti-viral among others, nutraceuticals as dietary supplements and food additives, agrochemicals with insecticidal, herbicidal, and fungicidal activities and cosmetics as photoprotective and anti-aging compounds. These compounds are believed to be superior to that derived from terrestrial nature in terms of their chemical novelty and their ability to induce potential activities [7]. They are mainly obtained from different marine taxonomic groups including antarctic fungi, bacteria, epiphytic bacteria and fungi, macroalgae or seaweeds, microalgae and mediterranean sponges [8].

In addition, advances in marine pharmacology have helped in the discovery of a lot of marinederived bioactive compounds. The real marine drug development has started with the discovery of Cephalosporin C in the 1940s from the mediterranean fungus Acremonium chrysogenum. It was the 
first compound in the antibiotic class of cephalosporin. Moreover, isolation of spongouridine and spongothymidine in the 1950s from the Caribbean sponge Tethya crypta led to the synthesis and approval of the more active nucleoside analogues cytarabine and vidarabine as potent anti-cancer and anti-viral drugs, respectively $[9,10]$. Some examples of these marine-derived products were summarized in Table 1.

Table 1: Selected categories and examples of marine-derived products

\begin{tabular}{|c|c|c|c|c|}
\hline Product category & Compound name & Biological source & Importance & Ref. \\
\hline \multirow[t]{15}{*}{ - Pharmaceuticals } & Mycosporine-Gly & Palythoa tubereulosa & \multirow[t]{2}{*}{ Anti-oxidant } & [11] \\
\hline & C-Phycocyanin & Spirulina platensis & & {$[12,13]$} \\
\hline & Fucoidan & $\begin{array}{l}\text { Brown macroalgae (e.g., } \\
\text { Fucus vesiculosus) }\end{array}$ & Anti-coagulant & {$[14]$} \\
\hline & $\begin{array}{l}\text { Eunicellane } \\
\text { diterpenoid }\end{array}$ & $\begin{array}{c}\text {-Klyxum molle } \\
\text {-Cladiella krempfi }\end{array}$ & Anti-inflammatory & [15] \\
\hline & Carotenoid & Padina boergesenii & Hepatoprotective & {$[16,17]$} \\
\hline & Axisonirile-3 & Acanthella klethra & Anti-malarial & {$[18]$} \\
\hline & $\alpha$-Kainic acid & $\begin{array}{l}\text {-Digenia sp. } \\
\text {-Sargassum sp. }\end{array}$ & Anti-parasitic & [19] \\
\hline & Vidarabine (Ara-A) & Tethya crypta & \multirow[t]{2}{*}{ Anti-viral } & \multirow[t]{2}{*}[10]{} \\
\hline & Avarol & Disidea avara & & \\
\hline & Cytarabine (Ara-C) & Cryptotheca crypta & \multirow[t]{3}{*}{ Anti-tumor } & {$[20]$} \\
\hline & Fucoxanthin & $\begin{array}{c}\text { Microalgae (e.g., } \\
\text { bacillariophytes) and brown } \\
\text { macroalgae (Laminaria } \\
\text { japonica) }\end{array}$ & & {$[21]$} \\
\hline & Borophycin & $\begin{array}{c}\text { Cyanobacteria (e.g., Nostoc } \\
\text { linckia and N. spongiaeforme } \\
\text { var. tenue) }\end{array}$ & & {$[22]$} \\
\hline & Chalcomycin & Streptomyces sp. HK-2006-1 & \multirow[t]{3}{*}{ Anti-microbial } & {$[23]$} \\
\hline & $\begin{array}{l}\text { Sesterterpene } \\
\text { sulphate }\end{array}$ & Dysidea sp. & & {$[24,25]$} \\
\hline & $\begin{array}{ll}\text { - } & \text { Cristatumins A } \\
\text { - } & \text { Cristatumins D }\end{array}$ & $\begin{array}{c}\text { Eurotium } \\
\text { cristatum } \text { EN-220 }\end{array}$ & & {$[26]$} \\
\hline
\end{tabular}


Cont., Table 1: Selected categories and examples of marine-derived products

\begin{tabular}{|c|c|c|c|c|}
\hline Product category & Compound name & Biological source & Importance & Ref. \\
\hline \multirow[t]{2}{*}{ - Cosmetics } & Scytonemin & Cyanobacteria sp. & $\begin{array}{l}\text { Topical photo- } \\
\text { protective }\end{array}$ & \multirow[t]{2}{*}{ [21] } \\
\hline & Astaxanthin & $\begin{array}{c}\text { Haematococcus } \\
\text { pluvialis }\end{array}$ & Anti-aging & \\
\hline \multirow{5}{*}{$\begin{array}{l}\text { - Nutraceuticals, } \\
\text { food additives and } \\
\text { dietary } \\
\text { supplements }\end{array}$} & Arachidonic acid & Porphyridium sp. & \multirow{2}{*}{$\begin{array}{l}\text { Polyunsaturated fatty } \\
\text { acids (PUFAs) }\end{array}$} & \multirow[t]{2}{*}{ [8] } \\
\hline & $\gamma$-Linolenic acid & Arthrospira sp. & & \\
\hline & Alginate & $\begin{array}{l}\text { Brown algae (e.g., } \\
\text { Laminaria sp.) }\end{array}$ & \multirow[t]{3}{*}{$\begin{array}{l}\text { Gelling, emulsifying } \\
\text { agents and stabilizers }\end{array}$} & {$[27,28]$} \\
\hline & Agar & $\begin{array}{l}\text { Red algae (e.g., } \\
\text { Gelidium amansii) }\end{array}$ & & [29] \\
\hline & Carrageenan & $\begin{array}{c}\text { Red algae (e.g., } \\
\text { Kappaphycus } \\
\text { alvarezii) }\end{array}$ & & {$[30,31]$} \\
\hline $\begin{array}{c}\bullet \text { Others } \\
-\quad \text { Enzyme inhibitors }\end{array}$ & Speradine A & Aspergillus tamari & $\begin{array}{c}\text { Inhibitory activity } \\
\text { against } \mathrm{Ca}^{2+} \text {-ATPase } \\
\text { and histone } \\
\text { deacetylase }\end{array}$ & {$[32]$} \\
\hline \multirow[t]{3}{*}{ - $\quad$ Source of enzymes } & Polyketide synthase & $\begin{array}{c}\text { Pseudoceratina } \\
\text { clavata }\end{array}$ & Polyketide synthesis & {$[33]$} \\
\hline & Alginate lyase & Microbulbifer sp. & Alginate degradation & {$[34]$} \\
\hline & Fucoidanase & $\begin{array}{l}\text { Dendryphiella } \\
\text { arenaria }\end{array}$ & $\begin{array}{l}\text { Degradation to low } \\
\text { molecular weight } \\
\text { fucoidan (LMWF) }\end{array}$ & [35] \\
\hline
\end{tabular}

As Table 1 showed, a lot of marine-derived products have been commercialized such as agar-agar, alginate, carrageenan...etc. However, few drugs succeeded to be approved by FDA and marketed such as ziconotide (Prialt ${ }^{\circledR}$ ) as a potent analgesic, trabectedin (Yondelis ${ }^{\circledR}$ ), and cytarabine or ara-C $\left(\right.$ Cytosar- $\left.\mathrm{U}^{\circledR}\right)$ as anti-tumour agents, vidarabine or ara-A (Vira- $\mathrm{A}^{\circledR}$ ) and iota-carrageenan $\left(\right.$ Carragelose $\left.^{\circledR}\right)$ as an anti-viral, and omega-3-acid ethylesters (Lovaza ${ }^{\circledR}$ ) which is a combination of ethyl esters of eicosapentaenoic acid (EPA) and docosahexaenoic acid (DHA) for treating hypertriglyceridemia [17]. Other compounds are still investigated in different pre-clinical and clinical phases and marketed shortly [36].

License of investigational pharmaceutical products for human use should obey the GMP guidelines in most of the world countries. The application of these principles assure production of a high-quality 
product, consistency between the investigational product and the future commercial product and thus reliability and the relevance of clinical trials to the product's efficacy and safety [37]. This low number of approved drugs reflected the presence of different challenges and problems to produce GMP-compliant bioproducts which include a sustainable supply without harmful ecological consequences, identification of a practical formulation, structural complexity and low therapeutic index of marine-derived compounds [36].

\subsection{Seaweeds}

Seaweeds or macroalgae have a long history of multiple applications as a human food (e.g., Laminaria japonica, Undaria pinnatifida, Pyropia sp. (formerly Porphyra) and Sargassum fusiforme) especially in the South-east countries, biofuels, source of various valuable products, in water purification from organic and inorganic wastes or a commercial source of hydrocolloids including agar, carrageenan and alginate. Interestingly, hydrocolloids' global annual production has recently reached 100,000 tons with a market value above US \$ 1.1 billion [38]. Moreover, according to FAO statistics (FAO, 2014, 2016), of the top seven most cultivated seaweed taxa, three were used mainly for hydrocolloid content: Eucheuma sp. and Kappaphycus alvarezii for carrageenans, and Gracilaria sp. for agar [39]. These benefits have urged the commercial market to find out competitive techniques to increase the global yield from seaweeds, such as sea farming or aquaculture and biotechnology. In 2014, the annual production of cultivated seaweeds reached 27.3 million tons, representing $27 \%$ of the total marine aquaculture production [40]. Fig. 1 shows the global production of seaweeds in tons aquaculture, according to the FAO, 2016 report [39]. In addition, a 2015 report from the market analyst Smithers Rapra expected that the global market for marine biotechnology or blue biotechnology for industrial applications has the potential to reach US $\$ 4.8$ billion by 2020 , rising to US $\$ 6.4$ billion by 2025 [41]. 


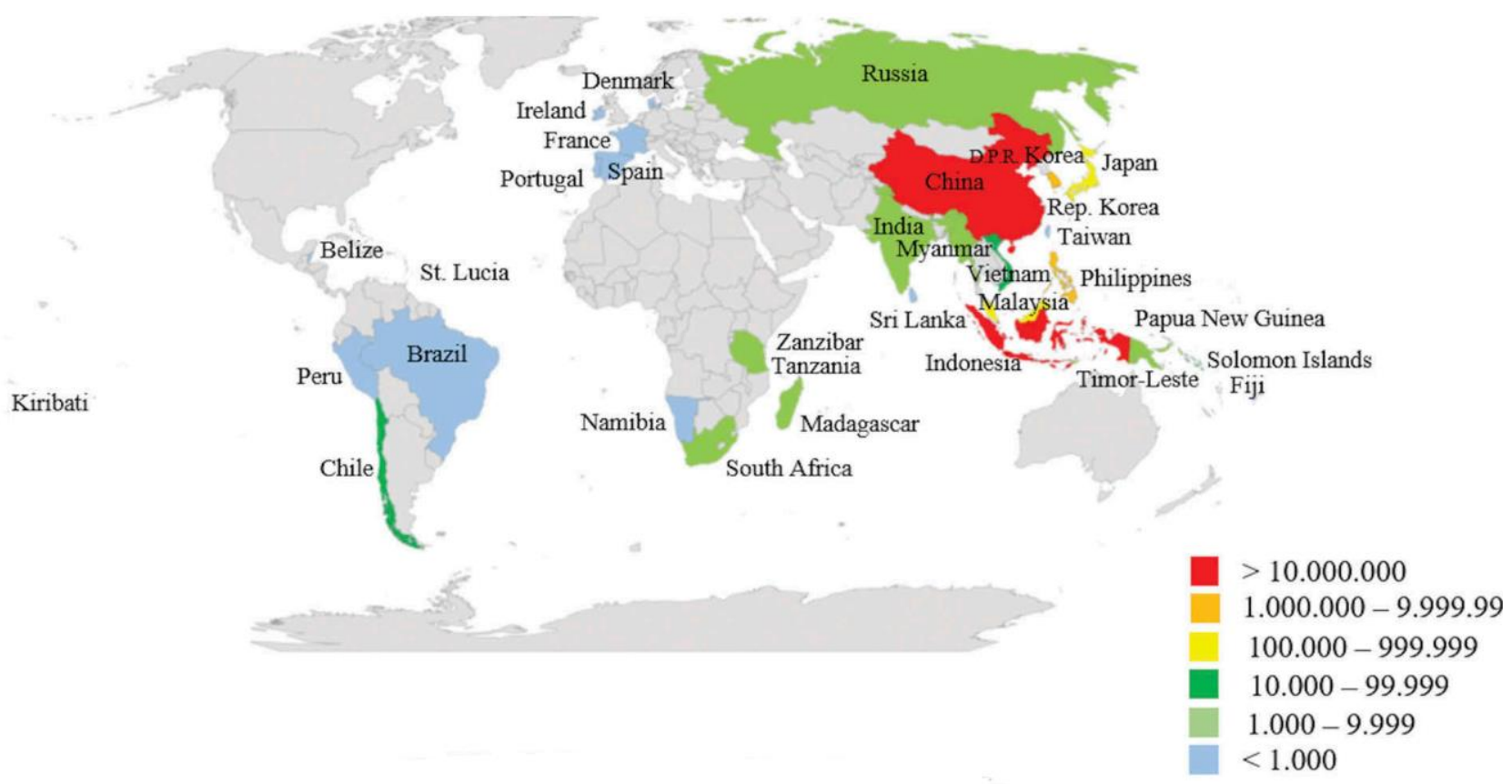

Fig. 1: Seaweed production in the year 2014 by Aquaculture [39]

Colour scale in wet metric tons (FAO, 2016).

Sulphated and non-sulphated polysaccharides from marine macroalgae have a wide-ranging of interesting medical applications, such as anti-coagulant and/or anti-thrombotic, immunomodulatory ability, anti-tumor, hypoglycemic, antibiotics, anti-inflammatory and anti-oxidant making them promising pharmaceutical products [42] including Phaeophytes or brown algae (e.g., fucoidan, laminarin and alginate), Rhodophytes or red algae (e.g., porphyrin and carrageenan) and Chlorophytes or green algae (e.g., rhamnans and ulvan). Unfortunately, structural and content heterogeneity of non-sulphated polysaccharides within the same genus resulted in poor investigation regarding chemical structure, biological activities and their structural-activity relationship (SAR) in comparison with sulphated analogues. Sulphated polysaccharides showed diverse molecular weights, monosaccharide compositions, sulphate contents and positions, which interact with various biological systems at different levels leading to diverse and interesting pharmacological activities $[42,43,44,45]$.

Particularly, brown macroalgae or Phaeophyceae consists of $c a .285$ genera and 1181 species [46] distributed not only on Fucales and Laminariales, which are the main commercial resources of the algal sulphated polysaccharides, but also contains Chordariales, Dictyotales, Dictyosiphonales, Ectocarpales, and Scytosiphonales. Fucales are one of the largest and most diversified orders within 
the class Phaeophyceae. They are composed of 8 families (41 genera and 485 species); namely the Ascoseiraceae, Cystoseiraceae, Durvillaeaceae, Fucaceae, Hormosiraceae, Himanthaliaceae, Sargassaceae, and Seirococcaceae [47]. As Fig. 2 shows, the major species of brown macroalgae are often dominant components of tropical to temperate marine forests and intertidal communities.

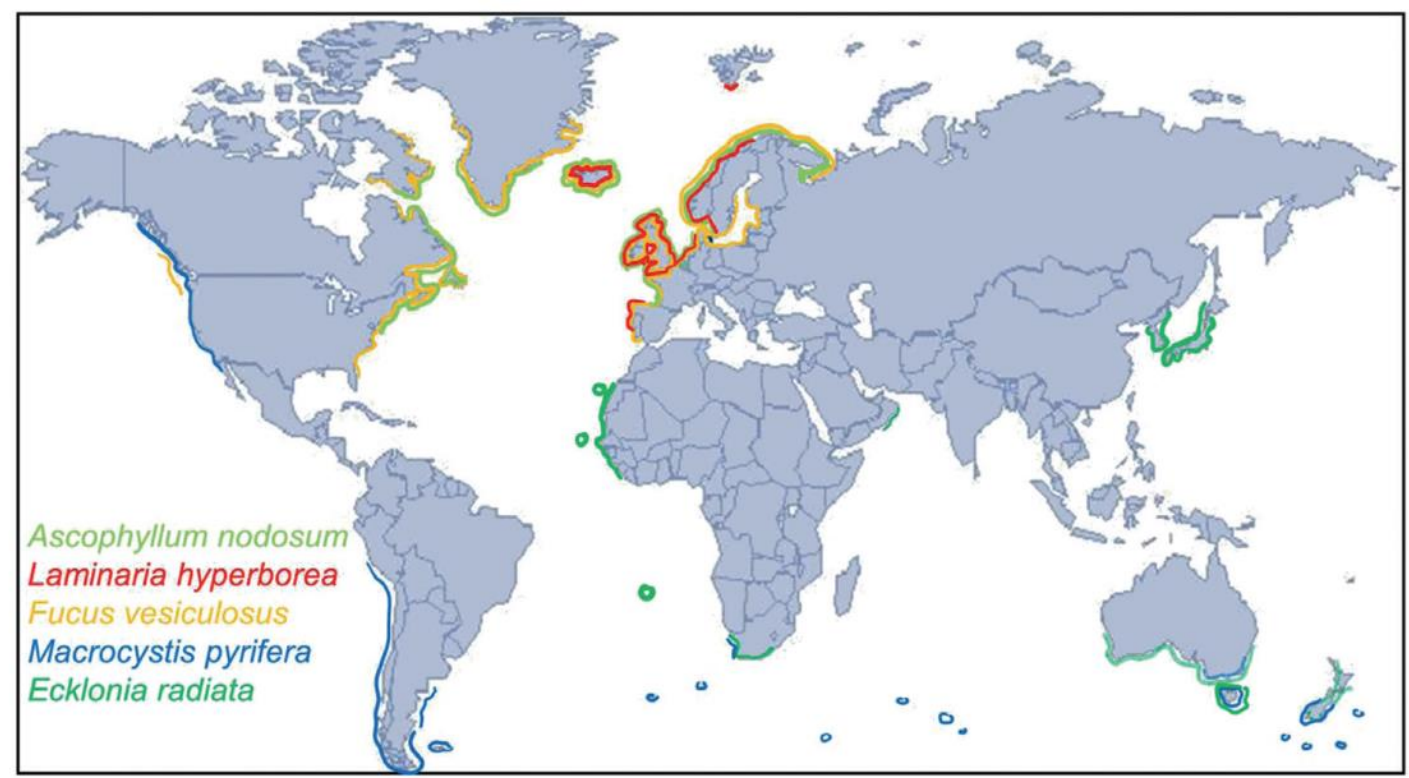

Fig. 2: Global distribution of the major brown macroalgae species [48]

\subsection{Fucoidan and its bioactivities}

Fucoidan is a marine polysaccharide isolated mainly from brown macroalagae with interesting and promising pharmacological activities [14]. Several articles discussed and proved potential, versatile and promising pharmacological activities of fucoidan, such as anti-coagulant and anti-thrombotic activity owing to structural similarity with heparin [49,50], anti-inflammatory [51], anti-oxidant [52], anti-viral against number of viruses like HSV [53], CMV [54] and HIV [55] ...etc., resulting in great interest in fucoidan in the last few decades as a candidate for drug discovery from nature with less side effects. Several features are involved and affect these activities, such as monosaccharide composition, molecular weight, sulphate esters content and sulphation pattern [56]. 
In more details, the following sections describe some of these activities.

\subsubsection{Anti-coagulant activity}

Physiologically, blood coagulation system or hemostasis functions through two cascades; namely extrinsic and intrinsic pathways, which are stimulated differently either by tissue injury or abnormal pathological conditions. However, they converged in a common pathway at the step of conversion of prothrombin to thrombin which catalyze the formation of the non-soluble fibrin blood clot. Extrinsic cascade is initiated as a response to a tissue or vascular injury that stimulates secretion of tissue factor or tissue thromboplastin from traumatized tissue. However, contact with exposed vessel wall or negatively charged surfaces, such as lipoprotein particles, release of phospholipids from activated platelets or even bacteria resulted in activation of contact or intrinsic coagulation pathway. Coagulation cascades are monitored through a number of clinical laboratory tests, such as APTT for the intrinsic pathway, PT for extrinsic pathway, and TT for common pathway.

Despite heparin has a long history to treat patients with thrombosis since 1940, it has suffered from a number of side effects including bleeding, thrombocytopenia in addition to possible pathogenic contamination from animal during production phases [57]. Several publications discussed the potential anti-coagulant activity of fucoidan $[49,50,58]$. They demonstrated that fucoidan acts in a heparin-like manner and interfered mainly with intrinsic pathway of coagulation system. It showed prolonged APTT and TT without a significant effect on PT. Moreover, not only negative charge distribution of fucoidan structure contributed to inhibition of thrombin, but also its enough long polysaccharide chain, high molecular weight, and structure comfortability were required for thrombin deactivation and discontinue of the fibrinogen conversion to fibrin [43].

\subsubsection{Anti-viral activity}

Fucoidan has a potential anti-viral activity against a number of enveloped viruses like HSV [53], CMV [54] and HIV [55] comparable to chemical drugs analogues such as ribavirin and acyclovir. Moreover, it has antiviral activity irrespective of whether these are DNA or RNA viruses [59]. Furthermore, Elizondo-Gonzalez, et al. proved the anti-viral activity of Cladosiphon okamuranus fucoidan against the enveloped virus Newcastle Disease Virus (NDV) in poultry field [60]. They found that fucoidan interferes with virus replication to inhibit viral-induced syncytia formation and cell-to-cell contact, possibly by blocking the fusion $(\mathrm{F})$ protein. It mediates fusion of the virus and 
cell membrane, an essential step for entry of the viral genome in the cell cytoplasm and initiation of a new infectious cycle [61]. Recently, fucoidan showed inhibitory activity against Hepatitis B Virus (HBV) replication by activation of the extracellular signal-regulated kinase (ERK) pathway, which leads to production of type I interferon (INF) [62]. Chemically, sulphate ester groups at C-4 of $\alpha$-(13)-linked fucopyranosyl units appeared to be involved in the anti-herpetic activity of fucoidan (HSV1 and HSV-2). This structure activity relationship was investigated in the brown seaweed Cystoseira indica fucoidan [63].

\subsubsection{Cytotoxic and anti-tumor activity}

Interestingly, a lot of researches discussed the potential anti-cancers activity of fucoidan [64]. Several fucoidans from Sargassum fulvellum, S. kjellmanianum, L. angustata, L. angustata var. longissima, L. japonica, Ecklonia cava, and Eisenia bicyclis have been found to have remarkable growth inhibitory activities against various types of tumors. Fucoidan induces cell apoptosis of several cancerous cell lines, such as melanoma, HT-29 colon cancer, MCF-7 human breast cancer, HS-Sultan human lymphoma through activation of different caspases-dependent pathways [65]. Other theories suggested activation of macrophages which resulted in production of cytokines such as IL-1, IL-2 and IFN- $\gamma$ with subsequent stimulation of T-cell [65].

\subsubsection{Anti-oxidant and radicle scavenging activity}

Antioxidants are medically beneficial compounds that fight against harmful reactive oxygen and free radicle species. Anti-oxidants are also important in food industry, where they prevent food deterioration. Despite potential fucoidan anti-oxidant activity against different free radicles (e.g., DPPH, superoxide, hydroxyl and lipid peroxides) [66] was discussed in many publications in vitro, most of these assays were investigated in crude fucoidan extracts [67,68,69], or/and its purified fractions [70]. It was observed that crude fucoidan had higher activity than its purified fractions and these findings indicated that co-extracted contaminants such as polyphenols interfere with this activity.

Nevertheless, its biogenic resources function potential ecosystem services and due to oppressive harvest from wild marine forests in the last decades, their populations in many regions around the world have declined and/or spatially shifted [48]. As a result, wild harvest was prohibited by many governmental legislations. In addition, fucoidan production and chemical structure are greatly 
affected by many other factors, such as seasonal and geographical factors as well as extraction methods which affect its pharmacological activities as a result. Therefore, production of a GMPcompliant product that could be investigated clinically is impossible without establishment of innovative and competitive techniques which guarantee a structurally-homogenous and eco-friendly supply.

\subsection{Goals of the work}

As seen in Fig. 3, The present dissertation deals with various aspects of fucoidan production, including optimization of fucoidan classical extraction and purification from marine crude algal extract to obtain a high-quality and native product, development of new biotechnological techniques without harmful effects on the global ecosystem through either callus and protoplast culture from brown algae or heterologous expression of enzymes involved in fucoidan biosynthesis (e.g., FucTs and STs). These routes of production should guarantee an improved yield of fucoidan with minimal structural differences in a commercial scale.

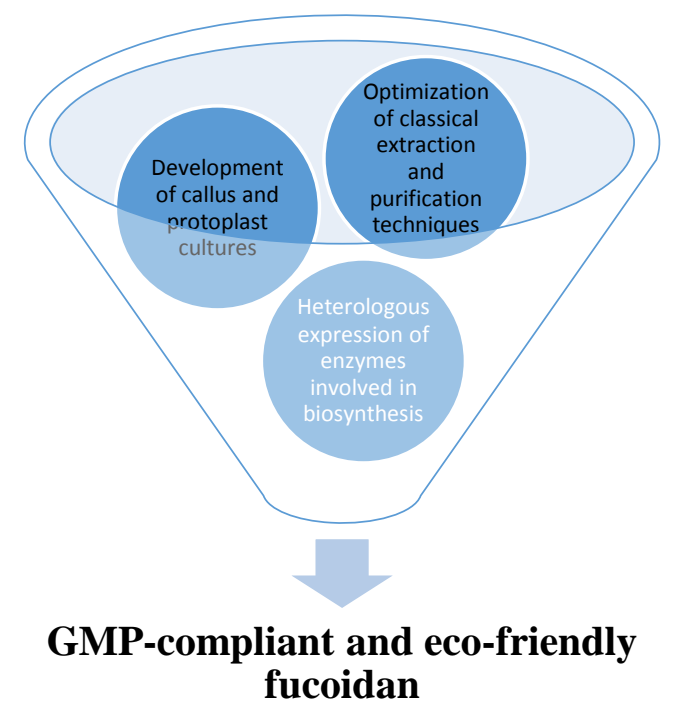

Fig. 3: Overview of the different strategies dealt in the present work

The techniques aimed to produce fucoidan that is compliant with GMP guidelines and from ecofriendly resources.

In details, the following aspects will be discussed within this thesis.

- Production of a GMP high-quality and native fucoidan in a simple, time-saving and cheap protocol is a challenging. To date, a standard and optimized protocol for extraction and 
purification techniques of fucoidan have not been developed yet. Recently, immobilized thiazine dyes succeeded in capture of fucoidan from a raw synthetic extract in a simple and fast procedure. However, this procedure has not been applied to real crude extracts and its effect on physico-chemical and pharmacological properties of fucoidan was not investigated. In Chapter 2, downstream processes applying different extraction and purification parameters (e.g., temperature, $\mathrm{pH}$, time) of fucoidan from $F$. vesiculosus were studied to isolate different fucoidan fractions applying the recently developed thiazine dyes-derivatized beads. These fractions were characterized physico-chemically (e.g., melting point, monomeric composition, and molecular weight) and pharmacologically (e.g., anti-coagulant, and anti-viral activities) afterwards. These studies aimed to reveal and understand some of fucoidan's SAR. In addition and for more effective purification, immobilization of the sensitive Heparin $\operatorname{Red}^{\circledR}$ (Redprobes UG) chemically-related PDD would be performed and applied to capture of fucoidan from a crude algal extract.

- In Chapter 3, growing of various cell cultures derived from F. vesiculosus was discussed. Development of closed-system bioreactors for seaweeds cultivation is a particularly challenging opportunity for marine bioprocess engineers [71]; where, cultivation in a closedsystem bioreactors ensures the growth of seaweeds at optimum conditions and production of consistent and homogenous value-added fucoidan as well. This type of production may minimize the structural heterogeneity, which are greatly affected with harvesting time and place, producing species and method of extraction. Since removal of marine microbes from macroalgae represents a rate-limiting step in the development of axenic tissue culture systems, a combination of various as well as delicate reagents should be optimally applied to establish a surface sterilization protocol and at the same time maintain explant viability. These axenic explants would be used in establishment of various types of growing cell phototrophic cultures.

- A novel technique for fucoidan production should be put into consideration; it is enzymatic synthesis. Heterologous expression of the enzymes involved in its biosynthesis in a bacterial cell line could open new chances to produce a high-quality and homogenous engineered fucoidan in vitro instead of classical extraction from its biogenic resources. The research in Chapter 4 aimed to clone some genes from the brown macroalge E. siliculosus to heterologous express their corresponding enzymes that are involved in fucoidan biosynthesis in E. coli BL21 (DE3) (e.g., FucTs and STs) 



\section{Optimization of Fucoidan Extraction and Purification from Brown Macroalgae}

\subsection{Introduction}

\subsubsection{Definition and functions}

Fucoidan is defined as a class of fucose-rich, water-soluble and sulphated homo- or heteropolysaccharides or fucose-containing sulphated polysaccharides (FCSPs) as it is commonly abbreviated. It exits mainly in the fibrillar cell walls and intercellular spaces especially of brown seaweeds (Phaeophyta) [65], urchins and marine invertebrates as well. It presumably functions in brown algae as a cross linker between cellulose and hemicellulose, and therefore is always accompanied with the polyphenolic phlorotannin to give the cell wall its integrity. Moreover, it has other roles in cell polarity and development, cell-to-cell communication and brown macroalgae defense mechanism, as shown in Fig. 4 [46,72]. Furthermore, fucoidan content was observed to be greatly affected by seasonal variations and tides level in the same organism, suggesting a possible protection role against organism desiccation $[58,73,74]$.

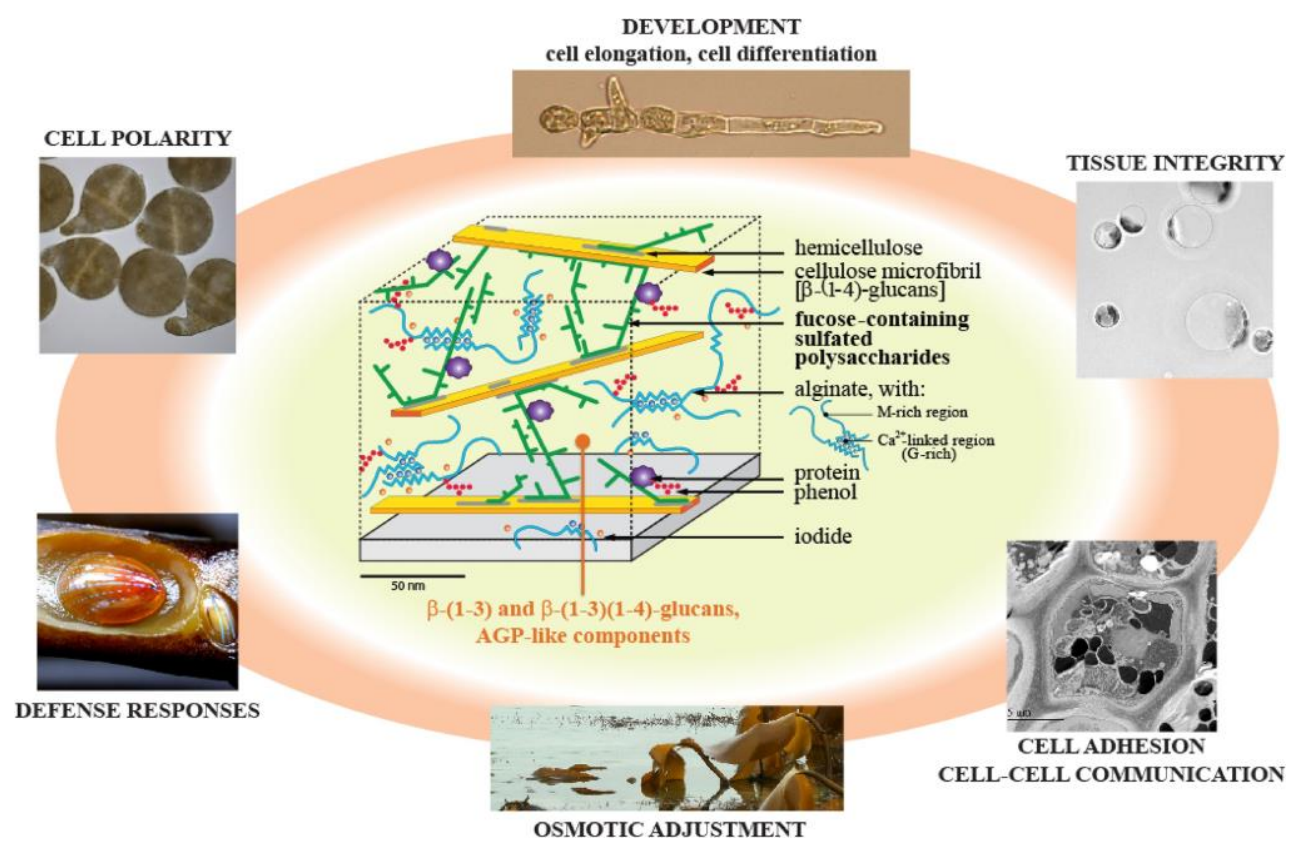

Fig. 4: Cell wall model in a brown macroalgae showing various fucoidan physiological functions

Fucoidan is involved in cell wall integrity, cell development, cell polarity, organism defense mechanism, osmotic adjustment and cell-to-cell communication [46]. 
In 1913, the Swedish scholar Kylin isolated fucoidan as a polymer of fucose for the first time (or fucoidin as he named it at this time) during his researches in the field of sea algae and fucosan content in different Fucus and Laminaria sp. extracts [75,76]. According to ISI web of knowledge (Clarivate Analytics), researches performed on fucoidan were scarce till 1980s. However, interest in fucoidan as a drug candidate has dramatically increased in the last few decades due to its chemical diversity, cheap and available resources and promising pharmacological activities, as shown in Fig. 5. This interest has resulted in 1833 published articles till July 2017 dealing with various aspects of fucoidan.

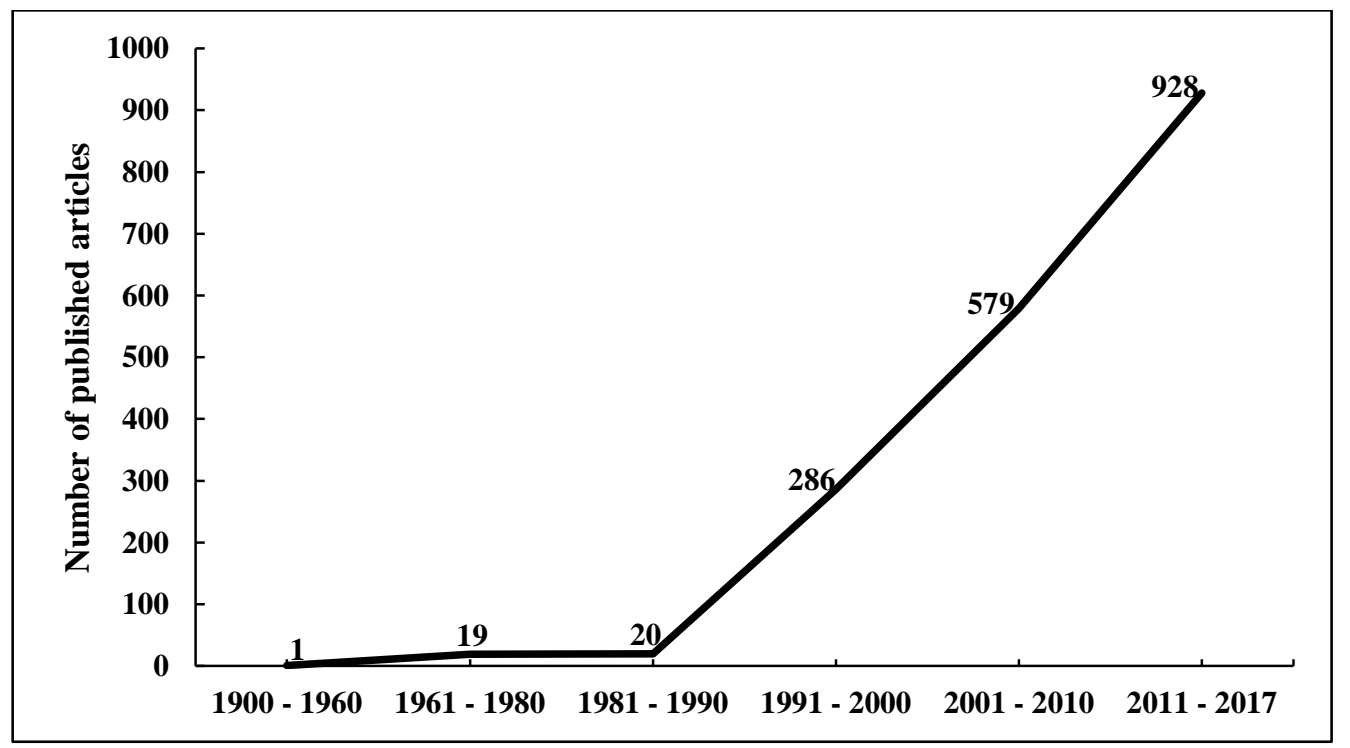

Fig. 5: Published articles on fucoidan since 1900 till July 2017, according to ISI web of knowledge (Clarivate Analytics)

It shows that a great interest has drawn in fucoidan since 1990s.

\subsubsection{Fucoidan chemistry}

Structural complexity and homogeneity of isolated fucoidan vary with its biological origin, for example; fucoidan of seaweeds showed commonly branching and more sulphated backbone with the presence of sugar monomers other than $\beta$-L-fucose. However, marine invertebrates' fucoidan such as sea cucumber is more simple, homogenous and composed of a linear-chain of repeating units $[46,77,78]$. These differences resulted in multiple biological activities of seaweeds' fucoidan making them a more preferable biogenic resource than marine invertebrates [79].

Literally, Cumashi et al. proposed that seaweeds' fucoidan is composed mainly from either $\alpha-(1-3)$ or alternating $\alpha-(1-3)$ with $\alpha-(1-4)$-linked sulphated linear or branched L-fucopyranoside backbone, based on their study of the chemical structures isolated from different brown seaweeds species. Other 
sugar units could be also present like mannose, xylose, galactose or even glucose in addition to uronic acids, but their positions and binding mode are still unclear. In addition, its fundamental subunit L-fucose is further mono- or di- sulphated or acetylated imparting a negative charge and anionic character on fucoidan molecular structure [14]. As shown in Fig. 6, fucoidan's chemical structures are between the different species of brown seaweeds and heterogenous regarding monomeric composition, glycosidic linkages and sulphation pattern. Moreover, its chemical structure might be affected by the applied extraction methods with the same organism $[65,76]$.

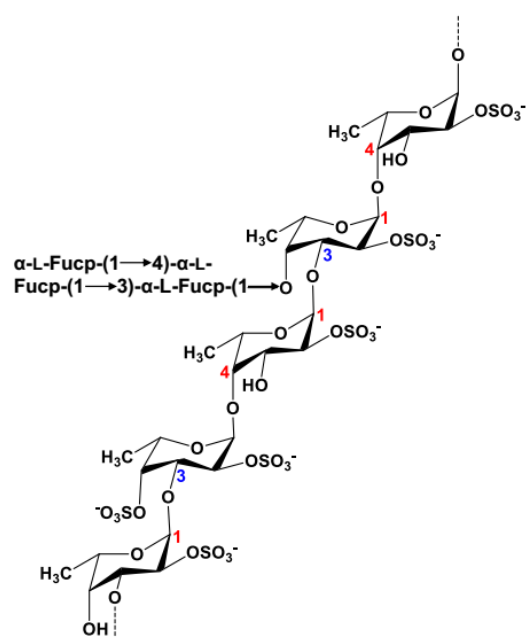

Fucus serratus $\mathbf{L}$.

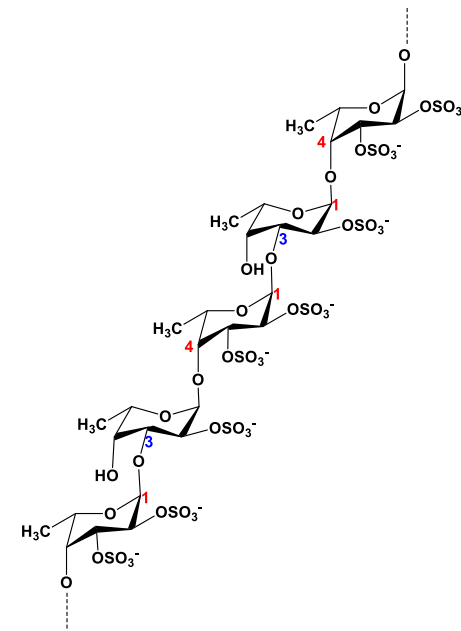

Fucus vesiculosus and Ascophyllum nodosum

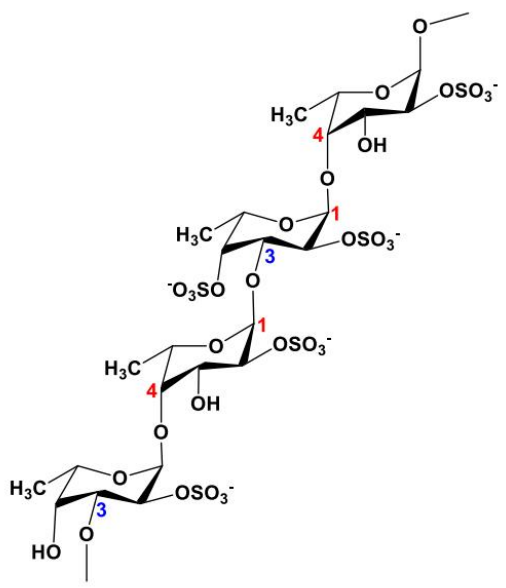

Fucus evanescens $\mathbf{C}$. Ag

Fig. 6a: Different chemical structures of fucoidan from some Fucales seaweeds [65]

They showed an alternating $\alpha-(1-4)$ and $\alpha-(1-3)$ linked L-fucopyranoside backbone. C-2 is usually substituted with sulphate ester groups in addition to alternating C-3 or C-4 in L-fucopyranose residue, according to the glycosidic linkages. In addition, branched chain polymers could be also found as in F. serratus. Other minor sugar units (e.g., mannose, galactose ...etc.,) share in fucoidan structure in certain unknown positions [14]. 


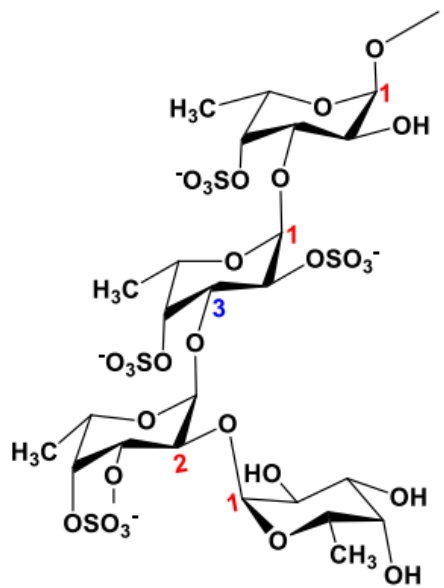

Laminaria saccharina (Laminariales)

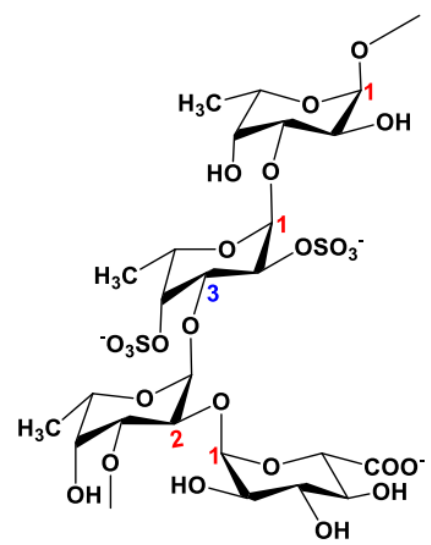

Cladosiphon okamuranus (Chordariales)

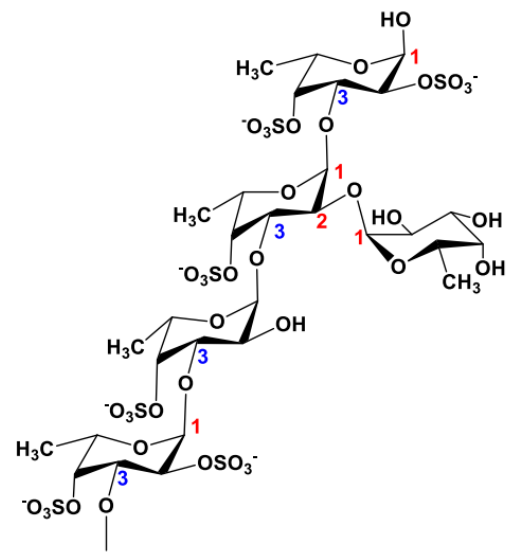

Chorda filum (Laminariales)

Fig. 6b: Chemical structures of fucoidan from some Laminariales and Chordariales seaweeds [65]

Both orders show repeated $\alpha$-(1-3) linked branched L-fucopyranoside backbone at C-2. Sulphate ester groups substitute mainly C-4 and sometimes C-2.

Due to its complex chemical structures, several spectroscopical methods (e.g., FTIR, NMR and MS) have been used to elucidate its structural features including position of sulphate groups, glycosidic bonds and molecular weight. Furthermore, application of regio- and stereo-selective enzymatic degrading fucoidanases isolated from marine bacteria provided new insight into the chemical structure of fucoidan $[43,74]$.

\subsubsection{Methods of extraction}

Since fucoidan is a water soluble polymer and insoluble in ethanol, aqueous extraction then its precipitation with ethanol are always applied. However, the process is not as simple as expected, due to the presence of fucoidan with many other intercalating components of the cell wall matrix (e.g., cellulose, hemicellulose, alginates, laminarin ... etc.). A standard extraction protocol for fucoidan has not yet been established, and therefore, different procedures at different $\mathrm{pHs}$ and temperatures, were applied to let protons or hydroxide ions to interfere with hydrogen bonds and then destruct the cell wall's matrix liberating fucoidan from the other polymers to the extraction medium $[44,65,78,79]$. These trials aimed to optimize extraction conditions and included extraction with hot or cold [80], acidic water ( $\mathrm{pH} 2.0$ - 2.3) [81], and alkaline water [82]. Using of $\mathrm{CaCl}_{2}$ was observed to be also advantageous during extraction for precipitation of alginate as Ca-alginate [46]. 
Recently, other novel extraction methods were applied utilizing hydrolytic enzymes (e.g., laminarinase, alginate lyase) under moderate conditions (EAE) [79,83], ultrasound (UAE) [84,85], and microwave (MAE) [86,87] for improving the rate, yield and selectivity. These techniques have also succeeded to eliminate the usage of chemicals and harsh extraction conditions and maintain the native nature of fucoidan.

For a more effective extraction, a pre-treatment step for the algal biomass after collection from beaches and coasts was performed. It included the removal of algal pigments (e.g., chlorophyll and fucoxanthin), lipids and other extraneous compounds which might co-extracted with fucoidan. This process included the treatment with acetone, methanol or methanol:chloroform:water $(4: 2: 1)$ $[79,88,89]$.

\subsubsection{Purification}

Even after pre-treatment, extraction of fucoidan from either brown macroalgae is, in most cases, accompanied with co-extracted contaminants (e.g., alginate, proteins, polyphenols,....etc.) and needs a purification step later on [79]. The presence of such impurities influences the biological activities of FCSPs, and therefore, it may delay full understanding of the biological activity of FCSPs [89] and physico-chemical properties as well. According to the ISI web of knowledge (accessed on 12.09.2017), only 95 published articles discussed the process of fucoidan purification from crude brown algae extracts, despite the word fucoidan was mentioned in 1865 articles representing $5 \%$ of the published articles. This survey indicated the difficulty and complexity of fucoidan purification by the available techniques and the need for other simpler novel techniques. Common examples of purification are described in the following sections.

\subsubsection{Exchange-based purification (Ion exchange chromatography, IEX)}

Anionic properties of fucoidan are involved in all techniques applied in fucoidan purification. Anionic resins (e.g., Diethylaminoethyl-cellulose (DEAE-C) or Diethylaminoethyl-sepharose) are usually applied, where negatively charged fucoidan can be exchanged with negative ions bound on the positively charged quaternary amino group of the diethyaminoethyl $[88,90]$. For fucoidan elution, a gradient concentration of $\mathrm{NaCl}$ is applied resulting in different fractions of fucoidan with different molecular weight, sulphate content and variables biological activities.

Disadvantages of this technique included contamination of eluted fucoidan with high percent of $\mathrm{NaCl}$ and other small anionic compounds that could be ionized at used $\mathrm{pH}$ such as amino acids, 
alginate and polyphenols. These contaminants required a further chromatographic step with GPC technique to remove them. Both chromatographic steps led to increase the costs of purification process. Furthermore, resins to be re-used, it should be regenerated making the process more tedious and time-consuming [91].

\subsubsection{Biologicals-based affinity chromatography}

Biologicals were also successfully applied as a tool for fucoidan purification, such as anti-thrombin III and heparin co-factor II, depending on the binding affinity of fucoidan in performing its anticoagulant function. These compounds were immobilized on concanavalin A-Sepharose and were applied to purify fucoidan [92]. More recently, fucose-specific lectins were applied in a single step to capture fucoidan similarly to fucose-containing proteins [43,93]. However, these techniques suffered also from several disadvantages, such as high expenses and inability of lectins to identify masked fucose units due to sulphate ester groups [94].

\subsubsection{Metachromasia-based affinity chromatography}

Thiazine cationic dyes (e.g., methylene blue and toluidine blue) are well-known in cell and tissue staining based on a metachromatic change from its blue to purple colour [95]. Moreover, analytical methods for detection and quantification of anionic polysaccharides were well-established through the formation of a charge transfer complex $[96,97,98]$. Hahn, et al. described the interaction between toluidine blue and the sulphated polysaccharide fucoidan, that it is strong enough and not only driven by ionic interaction, but also by disperse interactions between the stacked dye molecules [94,98]. In addition, TB was immobilized successfully on an amino derivatized Sepabeads ${ }^{\circledR}$ EC-EA by an innovative immobilization protocol through a glutaraldehyde bridge, as shown in Fig. 7. Furthermore, the adsorption kinetics and the binding capacity of the resin were analyzed. A Sips model was used to approximate the adsorption isotherm, resulting in a maximum loading capacity of $127.7 \mathrm{mg}$ fucoidan per $\mathrm{g}$ adsorbent [94]. TB-immobilized adsorbent could capture fucoidan in a cheaper, in comparison with IEX-GPC and immobilized biologicals, from a synthetic raw extract, in which similar substances to that present in the crude algal extract, such as lactose. $\mathrm{H}_{2} \mathrm{O}$, gluconic acid, gallic acid, and of BSA, were incorporated [94]. Yet, this procedure has not been investigated in crude fucoidan extracts. 

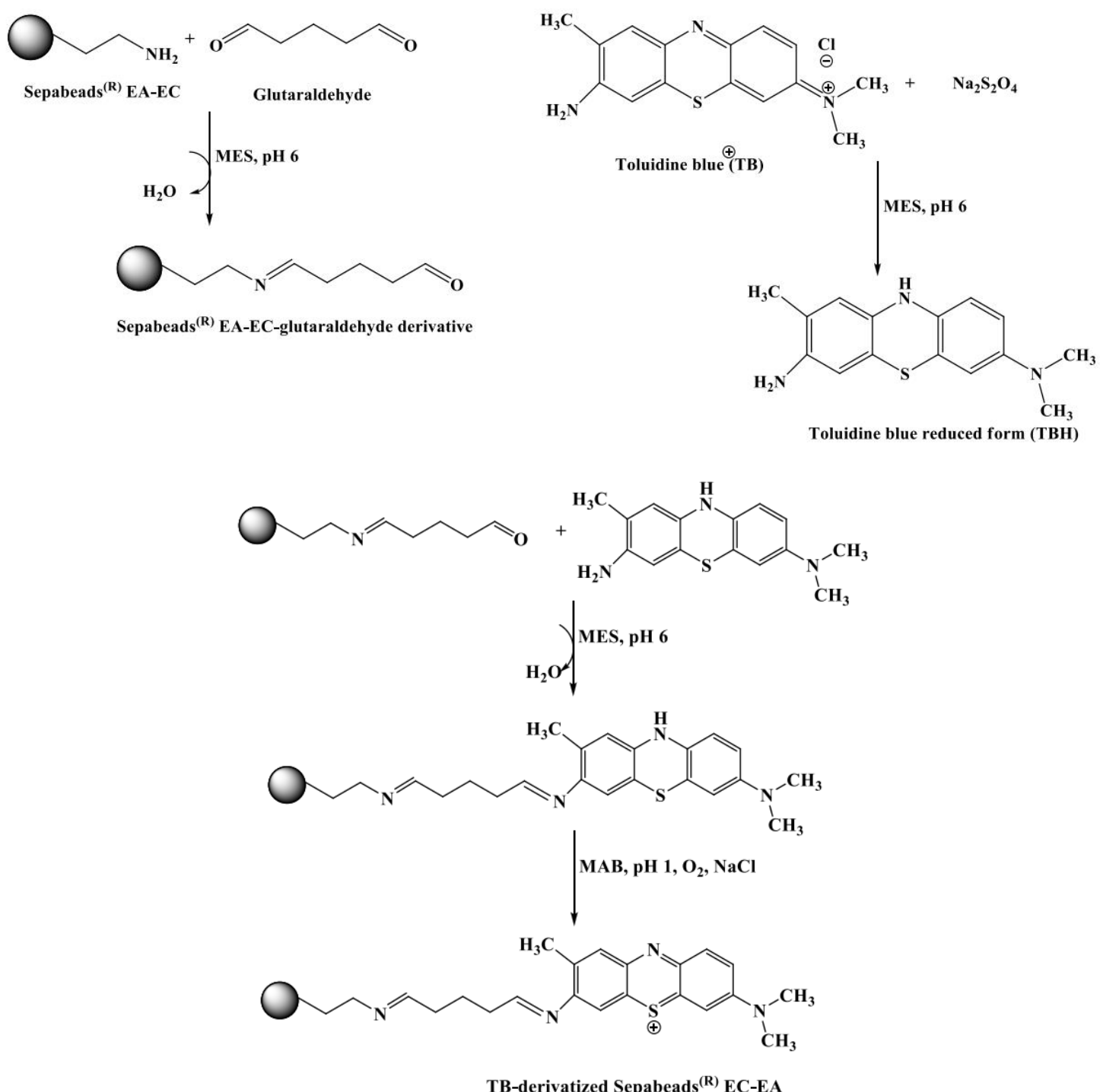

Fig. 7: Steps of TB immobilization protocol on amino derivatized Sepabeads ${ }^{\circledR}$ EC-EA [94]

The protocol involved derivatization of the beads with glutaraldehye, then a nucleophilic attack on carbonyl group of Sepabeads ${ }^{\circledR}$ EC-EA-glutaraldehye derivative was performed by TB in the presence of $\mathrm{Na}_{2} \mathrm{~S}_{2} \mathrm{O}_{4}$. Finally, a step pf oxidation at highly acidic $\mathrm{pH}$ produced the final form of the TBderivatized beads.

2.1.5. Fucoidan from $F$. vesiculosus or bladder wrack

As an example of fucalean fucoidan is the fucoidan isolated from $F$. vesiculosus or as commonly named bladder wrack. It is classified as GRAS substance and produced by many companies, such as Marinova (Maritech ${ }^{\circledR}$ ) [99] and Sigma-Aldrich ${ }^{\circledR}$. In comparison with the other fucoidan isolated from different brown algal species, it is characterized by its relatively simpler chemical structure. It 
is composed of $44.1 \%$ fucose, $26.3 \%$ sulfate and $31.1 \%$ ash, in addition to a little aminoglucose [43]. As shown previously in Fig. 6A, it is composed from repeating alternating $\alpha-(1-3)$ - with $\alpha-(1-4)$ linked L-fucopyranose units [14]. Therefore, fucoidan from F. vesiculosus was chosen as a model product for this work.

F. vesiculosus inhabits the littoral zone, where the tide changes the depth of the water, and the sublittoral zone, where the organism is constantly submerged around the north Atlantic, in more temperate waters with lower salinity for four to five years old, as shown in Fig. 8 [100]. In Nordic countries, two types of cultivation are available; onshore cultivation, where cultivation is established in tanks which is more expensive and offshore cultivation, where the cultivation is placed in the ocean with optimization of growth conditions. It is also possible to create the right conditions on the seabeds at intertidal, sheltered place with a wild population to settle and grow [101].

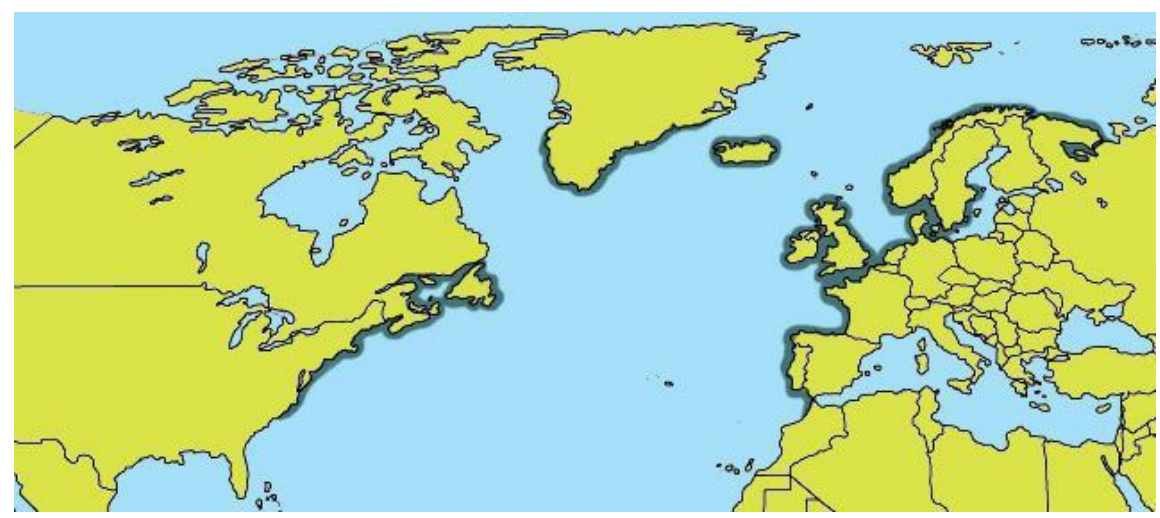

Fig. 8: Habitat of $F$. vesiculosus or bladder wrack across the north Atlantic in more temperate zone [100]

As a marine plant, the organism shows a number of morphological adaptations, such as the presence of a root-like structure holdfast which anchor the thallus to hard substrata such as pebbles, rocks, and dense seabeds. Moreover, fronds survive against sea waves by a flexible stipe and kept near to the water surface by means of vesicles or air bubbles found in lamina blades to continue the photosynthetic process. Fronds have also conceptacles at their tips which contain either one of the reproductive structures; antheridia or oogonia, as demonstrated in Fig. 9. Furthermore, taxonomy of F. vesiculosus could be described as followed in Table 2 [102,103]. 


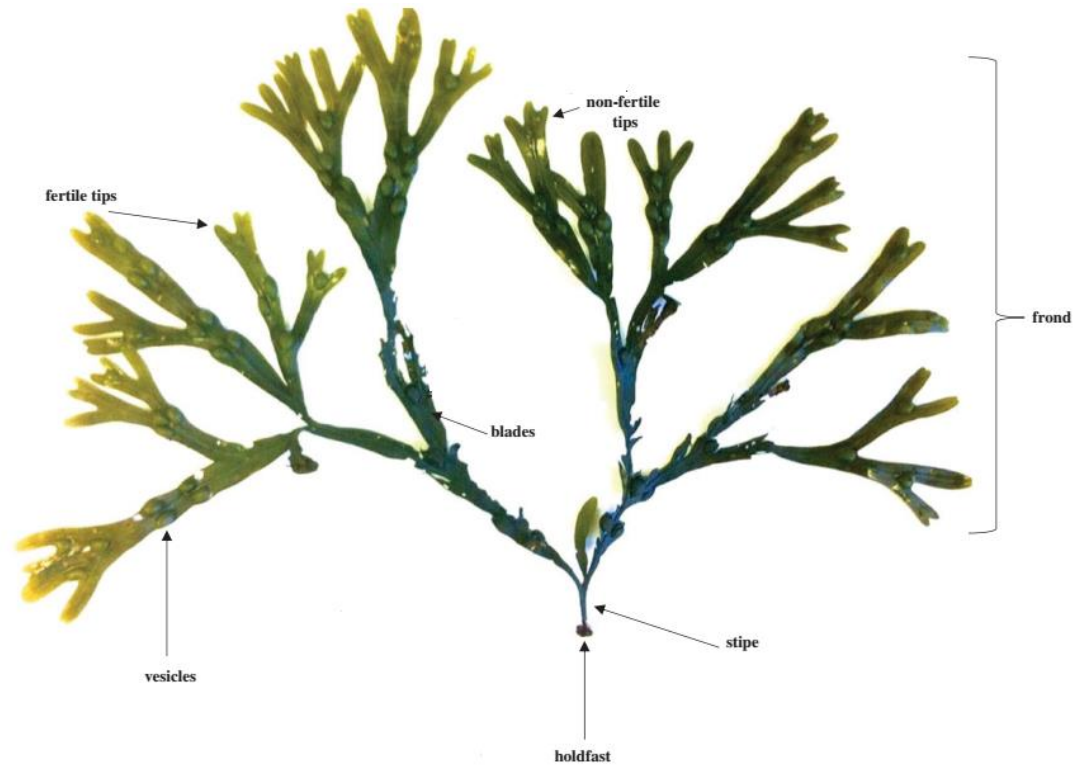

Fig. 9: Morphology and anatomical parts of $\boldsymbol{F}$. vesiculosus thallus (modified after [103])

Table 2: Taxonomy of $\boldsymbol{F}$. vesiculosus [102,103]

\begin{tabular}{|l|l|}
\hline Empire & Eukaryota \\
\hline Kingdom & Chromista \\
\hline Phylum & Ochrophyta \\
\hline Class & Phaeophyceae \\
\hline Subclass & Fucophycidae \\
\hline Order & Fucales \\
\hline Family & Fucaceae \\
\hline Genus & Fucus \\
\hline Species & vesiculosus \\
\hline
\end{tabular}




\subsection{Material and Methods}

2.2.1. Algae harvesting and pre-treatment

Fresh algal biomass of $F$. vesiculosus was harvested from the North Sea at the region of south beaches of Wilhelmshaven (Germany, 5331.236N, $8^{\circ} 13.849 \mathrm{E}$ ), as seen in Fig. 10. The algal biomass was washed with tap, and then deionized water, air-dried for few days, then in the drying oven at $50{ }^{\circ} \mathrm{C}$ until giving a constant dry weight, and milled afterwards. The milled algal powder was stored in a plastic container at room temperature.
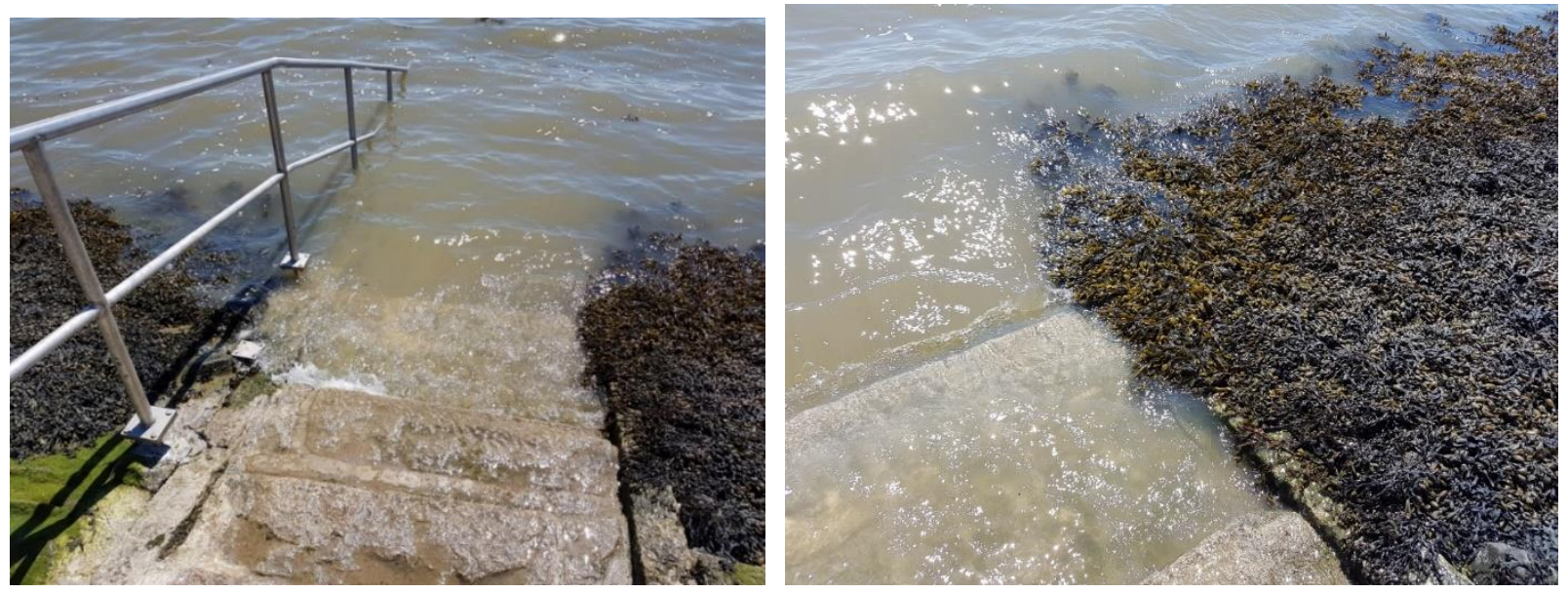

Fig. 10: Growth of the brown macroalgae $F$. vesiculosus at the south beaches of Wilhelmshaven (North Sea, $5^{\circ} 31.236 \mathrm{~N}, 8^{\circ} 13.849 \mathrm{E}$, Germany)

Before the extraction step and in a ration of 1:10 between the algal biomass and used solvent, except acetone step was 1:20, $10 \mathrm{~g}$ of a dried algal biomass were handled by several pre-treatment steps successively in a shaker incubator (Infors HT Ecotron) at a constant shaking rate (100 rpm) for overnight each at $25^{\circ} \mathrm{C}$ with of acetone, hexane : isopropanol (3:2), 80\% (v/v) ethanol, ethanol : water : formaldehyde (80:15:5) at $\mathrm{pH} 2.0$, and finally washed again with $80 \%(\mathrm{v} / \mathrm{v})$ ethanol. After each step, the suspension was centrifuged (4000 rpm, $10 \mathrm{~min}$ ) by a bench centrifuge, and the supernatant was decanted. The pretreated algal powder was then dried again at $60{ }^{\circ} \mathrm{C}$ and stored at room temperature in a well-closed plastic container. Scheme 1 demonstrates an overview of the major steps of pre-treatment. 


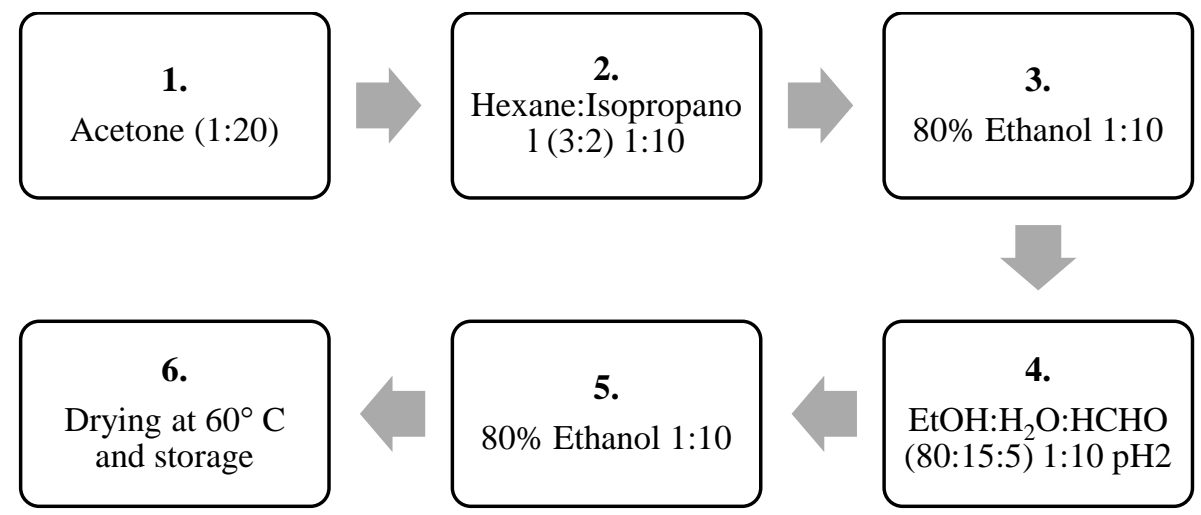

Scheme 1: Overview of pre-treatment steps of the dried algae biomass before fucoidan extraction

Steps were performed in a ration of 1:10 between the algal biomass and solvent, except acetone treatment in a shaker incubator a dusted at a constant rate $(100 \mathrm{rpm})$ for overnight each at $25^{\circ} \mathrm{C}$.

\subsubsection{Optimization of fucoidan extraction}

In an extraction set composed of a silicone oil bath over a magnetic heated stirrer and supplied with a thermometer and a temperature control device, as seen in Fig. 11, extraction was performed from the pre-treated $F$. vesiculosus biomass.

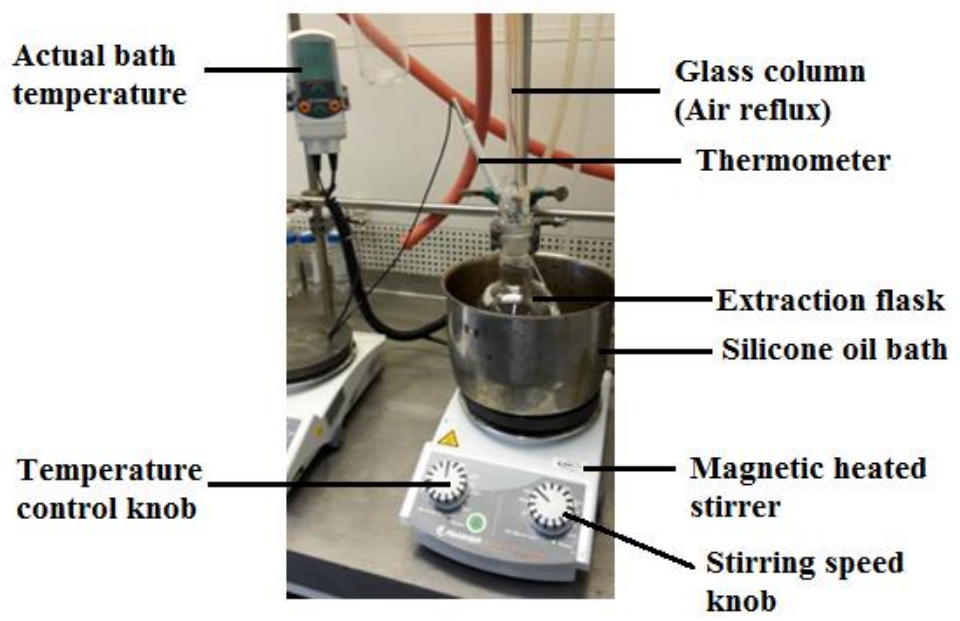

Fig. 11: Extraction set of fucoidan from dried pretreated $F$. vesiculosus

It shows an oil bath on heated magnetic stirrer and provided with a temperature control and thermometer. An air reflux column was applied in extraction Procedure A and B.

Three methods for fucoidan extraction were applied; Procedure A, B and C, which resulted in production of four fractions of crude fucoidan. Procedure A was performed by exhaustion for two times of $7 \mathrm{~g}$ of the pre-treated powder with $70 \mathrm{~mL}$ of $1 \%(\mathrm{w} / \mathrm{v})$ aqueous $\mathrm{CaCl}_{2}$ for $6 \mathrm{~h}$ at $70{ }^{\circ} \mathrm{C}$ using 
a reflux and continuous stirring (500 rpm) at $\mathrm{pH}$ 2.0, as described previously by Hahn, et al. [94]. The $\mathrm{pH}$ was adjusted regularly every $1-2 \mathrm{~h}$ at 1 by $1 \mathrm{M} \mathrm{HCl}$, as necessary. After centrifugation (4500 $\mathrm{rpm}, 15 \mathrm{~min}$ ), the algal biomass was removed and the supernatant was neutralized to $\mathrm{pH} 6.0$ by 2.0 M ammonium carbonate. Crude fucoidan was then isolated via precipitation by ethanol at a final concentration of $70 \%(\mathrm{v} / \mathrm{v})$, cooling overnight at $4{ }^{\circ} \mathrm{C}$, centrifugation, and then drying of the precipitate at $50{ }^{\circ} \mathrm{C}$ resulting in Fucoidan_A production. However, Procedure B administered $1 \%$ (w/v) $\mathrm{CaCl}_{2}$ in $20 \mathrm{mM} \mathrm{MAB}$ at $\mathrm{pH} 1$ as an extraction solvent. The extraction was carried out using the same conditions previously mentioned in Procedure A. Centrifugation was then used to separate the supernatant from the algal biomass. The supernatant containing Fucoidan_B was stored afterwards at $4{ }^{\circ} \mathrm{C}$ until the next step of purification. Procedure $\mathrm{C}$ was also performed at moderate conditions at $\mathrm{pH} 2.7$ at $42{ }^{\circ} \mathrm{C}$ for $3 \mathrm{~h}$ without reflux. Fucoidan_40\% and Fucoidan_70\% crude fucoidan fractions were isolated by precipitation with $40 \%$ (v/v) and 70\% (v/v) ethanol, respectively. As an overview for the extraction process and isolated crude fucoidan, Fig. 12 illustrated the whole process.

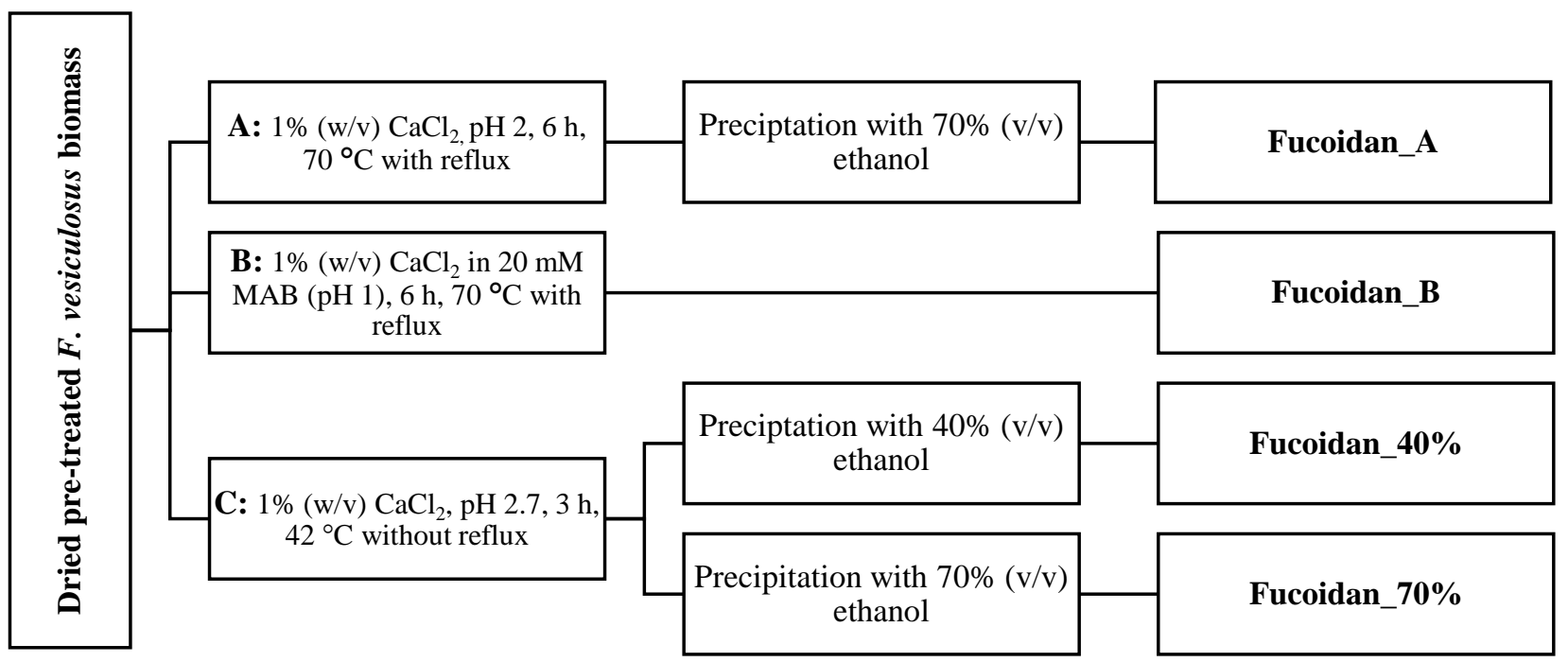

Fig. 12: Overview of fucoidan extraction process from pre-treated $F$. vesiculosus biomass and obtained crude fucoidan

Different fractions of crude fucoidan were isolated; namely Fucoidan_A, Fucoidan_B and Fucoidan_40\% and Fucoidan_70\%. 
2.2.3. Quantitative assay for crude fucoidan fractions' contents

Aqueous crude fucoidan solutions; Fucoidan_A, Fucoidan_(40\%) and Fucoidan_(70\%) were prepared in a concentration of $1 \mathrm{mg} \mathrm{mL}^{-1}$ and several contents were quantified (e.g., sugar, fucoidan, fucose and free sulphate contents) as described in the following sections.

\subsubsection{Sugar content}

According to Dubois and Holtkamp, Dubois or Phenol-Sulphuric acid assay was performed $[104,105]$. At the beginning, $200 \mu \mathrm{L}$ of each solutions were mixed with $200 \mu \mathrm{L}$ of a $5 \%$ (w/v) phenol solution gently in a $1.5 \mathrm{~mL}$ reaction vessel. Afterwards, $1 \mathrm{~mL}$ of concentrated sulphuric acid was added carefully and gently. After $10 \mathrm{~min}$, the samples were mixed vigorously for about $15 \mathrm{sec}$ and $30 \mathrm{~min}$ later at room temperature, the absorbance was measured at $490 \mathrm{~nm}$ by a Uv/Vis-spectrometer. Different concentrations $\left(0.025-0.25 \mathrm{~g} \mathrm{~L}^{-1}\right)$ of aqueous glucose solution were measured as standards.

\subsubsection{Fucoidan content}

Toluidine blue assay was performed according to Hahn, et al. [98]. Briefly, $10 \mu \mathrm{L}$ of fucoidan containing solutions were mixed with $990 \mu \mathrm{L}$ of $0.06 \mathrm{mM}$ TB which was prepared in $20 \mathrm{mM} \mathrm{MAB}$ (pH 1) for a better reaction sensitivity. Absorbance was afterwards measured at $632 \mathrm{~nm}$ using the aqueous solution of commercially-available fucoidan (>95\% pure) purchased from Sigma-Aldrich ${ }^{\circledR}$ as a reference standard in a concentration range of $0-2.5 \mathrm{~g} \mathrm{~L}^{-1}$.

Moreover, Heparin $\operatorname{Red}^{\circledR}$ Ultra assay, according to Warttinger, et al., was carried out for Fucoidan_A [106] after some modifications. In brief, $5 \mu \mathrm{L}$ of the fucoidan-containing sample were pipetted into a 96 microplate well. Then, $180 \mu \mathrm{L}$ of Heparin $\operatorname{Red}^{\circledR}$ Ultra solution was added as quickly as possible. The microplate was immediately placed in the fluorescence microplate reader adjusted at an excitation $\lambda$ at $570 \mathrm{~nm}$ and emission was recorded at $605 \mathrm{~nm}$ with a spectral band width $13.5 \mathrm{~nm}$ and read height of $8 \mathrm{~mm}$. Mixing was performed using the plate shaking function (setting "high", $1 \mathrm{~min}$ ) and fluorescence was measured within one minute after mixing. Fucoidan from Sigma-Aldrich ${ }^{\circledR}$ was used as standard $\left(0-8 \mu \mathrm{g} \mathrm{mL}^{-1}\right)$.

\subsubsection{Fucose content}

Dische or cysteine- $\mathrm{H}_{2} \mathrm{SO}_{4}$ assay was performed to quantify L-fucose content in hydrolyzed fucoidan solutions [105,107]. At first, $400 \mu \mathrm{L}$ of crude fucoidan solutions were mixed with $1.8 \mathrm{~mL}$ diluted sulphuric acid (1:6). The mixtures were subsequently cooked in a silicone oil bath at $100{ }^{\circ} \mathrm{C}$ for a period of $10 \mathrm{~min}$ and the reaction was stopped by cooling in an ice bath. Thereafter, $40 \mu \mathrm{L}$ of an 
aqueous 3\% (w/v) L-cysteine. $\mathrm{HCl}$ solution was added and the absorbance was measured at $396 \mathrm{~nm}$ and $430 \mathrm{~nm}$. With the difference of those two measurements the influence of other sugars could be neglected. As a standard, aqueous L-fucose solutions were used in a concentration range of $0.03-$ $0.21 \mathrm{~g} \mathrm{~L}^{-1}$.

\subsubsection{Free sulphate content}

Using $\mathrm{BaSO}_{4}$ assay, free or hydrolyzed suphate ester content was determined [108]. In a $20 \mathrm{mM}$ MAB (pH 2), aqueous $5 \mathrm{~g} \mathrm{~L}^{-1}$ crude fucoidan and $10 \%(\mathrm{w} / \mathrm{v}) \mathrm{BaCl}_{2}$ solutions were prepared. $250 \mu \mathrm{L}$ of each crude fucoidan solutions were mixed with $500 \mu \mathrm{L}$ of $10 \%(\mathrm{w} / \mathrm{v}) \mathrm{BaCl}_{2}$. After incubation on ice for $15 \mathrm{~min}$, absorbance at $600 \mathrm{~nm}$ was measured. A concentration range $\left(0.1-0.6 \mathrm{~g} \mathrm{~L}^{-1}\right)$ of $\left(\mathrm{NH}_{4}\right)_{2} \mathrm{SO}_{4}$ was used as a standard for calibration.

2.2.4. Perylene diimide derivative synthesis (PDD)

Synthesis of the red fluorescent PDD was performed at the Institute of Inorganic Chemistry in Heidelberg University by Prof. Dr. Roland Krämer as described previously by Szelke, et al. [109]. In brief, 1,7-dibromoperylene-3,4,9,10-tetracarboxylic acid dianhydride was converted to the diimide derivative by reaction with tris-(t-Butoxycarbonyl) protected by tetraamine spermine. After deprotection with trifluoroacetic acid, the product was isolated as a trifluoroacetate salt.

2.2.5. Immobilization of thiazine dyes and perylene diimide derivative [108]

Thiazine dyes (e.g., toluidine blue and thionin acetate) and PDD were immobilized according to the protocol described by Hahn, et al. [94] on Sepabeads ${ }^{\circledR}$ EC-EA purchased from Resindion S.R.1, Italy. The beads are porous ethyl amino-derivatized polymethacrylate enzyme carrier [110]. Steps were described in details in Appendix D: Protocols.

2.2.6. Adsorption kinetics in crude fucoidan

Applying the same conditions previously used in section 2.2.6, Fucoidan_A instead of the commercial fucoidan was incubated with $75 \mathrm{mg}$ of TB- and PDD derivatized beads. After 1080, 1440, 2400 and $2640 \mathrm{~min}$ for immobilized TB, and 5, 60 and $960 \mathrm{~min}$ for immobilized PDD, a sample volume of $10 \mu \mathrm{L}$ were analyzed by TB assay and percent of adsorbed fucoidan was calculated based on the decrease in fucoidan concentration in the incubation supernatant. 
2.2.7. Fucoidan purification and optimization

\subsubsection{Batch process}

Immobilized TB and PDD were applied to purify fucoidan from the isolated Fucoidan_A crude fraction following Hahn's previously-described protocol which was applied in a raw synthetic extract [108]. Four phases were executed including adsorption, washing, elution and recovery of fucoidan from eluate. Briefly, $50 \mathrm{mg}$ of derivatized beads in a $2 \mathrm{~mL}$ reaction vessel was incubated with $1.5 \mathrm{~mL}$ of $2.5 \mathrm{mg} \mathrm{mL}^{-1}$ of crude fucoidan. Then, two steps of washing with deionized water and $0.1 \mathrm{M} \mathrm{NaCl}$ in $20 \mathrm{mM} \mathrm{MAB} \mathrm{(pH} \mathrm{2)} \mathrm{and} \mathrm{elution} \mathrm{with} \mathrm{NaCl}$ prepared in 30\% (v/v) ethanol in MES (pH 6) were performed. In more details, steps of fucoidan purification and recovery were described in Appendix D: Protocols. Moreover, Different factors which may affect fucoidan adsorption and elution were studied. At the same time, different purified fucoidan fractions were produced to investigate the effect of purification conditions on fucoidan's physico-chemical and pharmacological properties. These factors and isolated purified fractions were discussed in the following sections.

a. Incubation $\mathrm{pH}$

Stock solutions of a $2.5 \mathrm{mg} \mathrm{mL}^{-1}$ concentration were prepared in buffers of different $\mathrm{pH}$ values, such as $20 \mathrm{mM} \mathrm{MAB} \mathrm{(pH} \mathrm{1)} \mathrm{and} 20 \mathrm{mM}$ MES (pH 6) to study the effect of incubation $\mathrm{pH}$ on percent capture and molecular weight of isolated fucoidan. The incubation with $50 \mathrm{mg}$ of TB-derivatized beads produced Fucoidan_1 and Fucoidan_6 as a result from incubation at pH 1 and pH 6, respectively. However, purification with PDD-derivatized beads at pH 6 resulted in Fucoidan_PDD.

b. Type and molarity of thiazine dye

In addition to $2 \mathrm{mM}$ of TB, TA was immobilized for a purpose of comparison. Moreover, different molarities of TB and TA $(2,4$ and $6 \mathrm{mM})$ were immobilized on Sepabeads ${ }^{\circledR}$ EC-EA. Mixed beads were also applied to capture fucoidan. These factors were studied using the same condition applied in $\mathrm{pH}$ factor.

c. Presence of interferences and skipping of precipitation step

Fucoidan_B was purified by TB-derivatized beads using the same conditions. This step produced a fourth purified fraction; namely Fucoidan_M.

d. Quantity of derivatized beads

Derivatized beads (50 mg and $75 \mathrm{mg}$ ) were compared regarding their loading capacity for capturing fucoidan from crude Fucoidan_A in MES (pH 6). 
e. Multiple use of beads

TB- and PDD-derivatized beads (75 mg) were used for two cycles to compare between beads' loading capacity for $44 \mathrm{~h}$ and $16 \mathrm{~h}$, respectively. The $2^{\text {nd }}$ cycle was performed after elution of adsorbed fucoidan from the $1^{\text {st }}$ cycle.

f. NaCl molarity in eluent

Different eluents with 1, 2 and $3 \mathrm{M} \mathrm{NaCl}$ were prepared in $30 \%(\mathrm{v} / \mathrm{v})$ ethanol in $20 \mathrm{mM} \mathrm{MES}(\mathrm{pH}$ 6). All eluents were applied for $16 \mathrm{~h}$ using a thermoshaker at $50{ }^{\circ} \mathrm{C}$ and $800 \mathrm{rpm}$. $\mathrm{NaCl}$ molarity was investigated to show its relationship with eluted fucoidan (\%) and fucoidan molecular weights.

\subsubsection{FPLC process}

For a scale-up and an automated purification process, a FPLC was used to establish a protocol that had the same optimized phases as in the batch process. $3.2 \mathrm{~g}$ of previously-conditioned with MES (pH 6) PDD-derivatized beads were packed in a class column (XK 16/20, 72x16 mm, $4 \mathrm{~cm}^{3}$ ), then $1 \mathrm{~mL}$ of $50 \mathrm{mg} \mathrm{mL}^{-1}$ in MES of crude Fucoidan_A fraction was injected. Afterwards, different flow rates and volumes were applied during the purification phases as shown in Table 3.

Table 3: Description of an automated fucoidan purification process by immobilized PDD using FPLC Different eluents with different flow rates and volumes were applied during the purification phases.

\begin{tabular}{|l|l|l|l|}
\hline \multicolumn{1}{|c|}{ Step } & \multicolumn{1}{|c|}{ Eluent used } & \multicolumn{1}{|c|}{$\begin{array}{c}\text { Flow rate } \\
\left(\mathbf{m L ~ m i n}^{-1}\right)\end{array}$} & \multicolumn{1}{|c|}{$\begin{array}{c}\text { Step volume } \\
(\mathbf{m L})\end{array}$} \\
\hline Column conditioning & $20 \mathrm{mM}$ MES (PH 6) & 1.0 & 15 \\
\hline Sample injection & $\begin{array}{l}50 \mathrm{mg} \cdot \mathrm{mL}^{-1} \text { Fucoidan_A in 20 mM } \\
\text { MES (pH 6) }\end{array}$ & 1.0 & 1.0 \\
\hline Adsorption & $20 \mathrm{mM} \mathrm{MES} \mathrm{(pH6)}$ & 0.5 & 30 \\
\hline Washing & Deionized water & 1.0 & 15 \\
\cline { 2 - 4 } & $0.1 \mathrm{M} \mathrm{NaCl}$ in 20 mM MAB (pH 2) & 1.0 & 20 \\
\hline Elution & $\begin{array}{l}3 \mathrm{M} \mathrm{NaCl} \text { in 30\% v/v ethanol in 20 mM } \\
\text { MES (pH 6) }\end{array}$ & 2.0 & 50 \\
\hline
\end{tabular}

Effluents of $2 \mathrm{~mL}$ fraction were collected during flow through, washing and elution phases which analyzed by TB assay for fucoidan content. The process was repeated for two times. This process produced two further fractions of purified fucoidan which named Fucoidan_PDD_1 and Fucoidan_PDD_2 from the $1^{\text {st }}$ and $2^{\text {nd }}$ cycle, respectively. Fig. 13 summarized the whole purification process and the different fractions obtained from crude fucoidan fraction. 


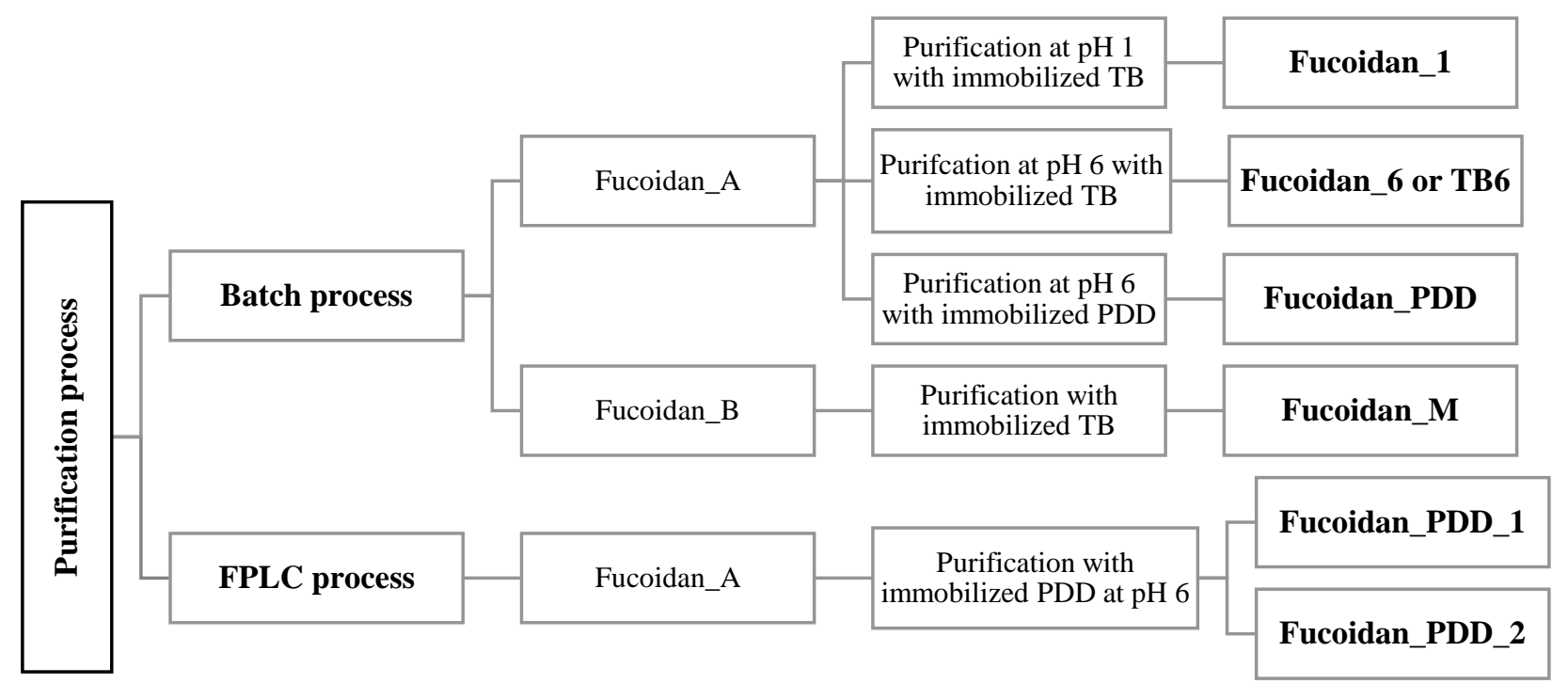

Fig. 13: Overview for isolated fractions of purified fucoidan

Six fractions were obtained from crude fucoidan using batch and FPLC processes.

2.2.8. Purified fucoidan fractions characterization

\subsubsection{Physico-chemical investigations}

Several investigations were carried out to characterize the isolated purified fucoidan fractions and evaluate the different studied factors in comparison with crude fucoidan (Fucoidan_A) and the commercially-available fucoidan $(>95 \%)$ purchased from Sigma-Aldrich ${ }^{\circledR}$ isolated from $F$. vesiculosus. These investigations were performed as described in the following sections.

a. Elemental (CHNS) analysis

Samples of 1.6 to $1.8 \mathrm{mg}$ or higher, especially in case of low element percent values, in tin boats were combusted. Combustion gases were reduced by hot copper at $830{ }^{\circ} \mathrm{C}$ in the reduction oven, then separated in the absorption column into its single components by temperature programmed desorption and the components were transferred sequentially to the thermal conductivity detector. The processor unit calculated afterwards the element concentrations from the measured values and the sample weight based on an instrument calibration. Elemental analysis was performed by the elemental Vario Micro cube apparatus which was calibrated previously with sulphanilic acid.

b. Molecular weight parameters

Molecular weight measurements were performed using an isocratic HPLC-GPC system. Samples were dissolved in phosphate buffer $\left(0.05 \mathrm{M} \mathrm{Na}_{2} \mathrm{HPO}_{4}, \mathrm{pH} 9.1\right)$ in a concentration of $4 \mathrm{mg} \mathrm{mL}^{-1}$ and 
then diluted with an equal volume of $1 \mathrm{mg} \mathrm{mL}^{-1}$ ethylene glycol solution as a flow marker. Separation was performed at $25^{\circ} \mathrm{C}$ using a GPC_MCX column, which was previously calibrated with dextran of GPC analytical standard grade of different molecular sizes (5.0-670 kDa). The injection volume was $10 \mu \mathrm{L}$ and the volumetric flow rate $1 \mathrm{~mL} \mathrm{~min}^{-1}$. The signal detections were performed using RI detector.

\section{c. Melting point}

Two mg of each fraction were placed in a capillary tube and placed in the melting point apparatus. Temperature increment was $2{ }^{\circ} \mathrm{C} \mathrm{min}^{-1}$ and three temperature points, at which solids started to melt, changed their colour to dark brown, and finally at complete decomposition with charring, were observed and recorded.

d. Specific optical rotation

An aqueous $0.4 \%(\mathrm{w} / \mathrm{v})$ solution of each fucoidan fraction was prepared and the specific optical rotation was measured at $22{ }^{\circ} \mathrm{C}$ by a digital polarimeter supplied with a sodium spectral adjusted at $\lambda 589 \mathrm{~nm}$.

\section{e. Monomeric composition}

Monomeric composition was determined according to protocols developed by Rühmann, et al. [111]. Briefly, $1 \mathrm{mg} \mathrm{mL}^{-1}$ aqueous solution of each fucoidan fractions was prepared and hydrolyzed with $2 \mathrm{M}$ trifluroacetic acid for $90 \mathrm{~min}$ at $121{ }^{\circ} \mathrm{C}$. Sugar monomers were detected, afterwards, as PMP derivatives with a HPLC-UV-ESI-MS system. Tempered column to $50{ }^{\circ} \mathrm{C}$, an autosampler to $20{ }^{\circ} \mathrm{C}$, a flow rate of $0.6 \mathrm{~mL} \mathrm{~min}^{-1}$ and an injection volume of $10 \mu \mathrm{L}$ were used. Mobile phase A and B consisted of $5 \mathrm{mM} \mathrm{CH}_{3} \mathrm{COONH}_{4}(\mathrm{pH} 5.6)$ with $15 \%$ acetonitrile and pure acetonitrile, respectively. The gradient was programmed as following: start of mobile phase B at $1 \%(\mathrm{v} / \mathrm{v})$, with increase to $5 \%$ over $5 \mathrm{~min}$, hold for $2 \mathrm{~min}$, with following increase to $18 \%$ over $1 \mathrm{~min}$. The gradient was further increased to $40 \%$ over $0.3 \mathrm{~min}$, hold for $2 \mathrm{~min}$ and returned within $0.2 \mathrm{~min}$ to starting conditions for 1.5 min. Before entering ESI-MS the flow was splitted 1:20. Fucose, xylose, and galactose were detected by the diode array detector, while glucose and uronic acid dimers were detected by MS detector.

\section{f. FT-IR spectrometry}

Two to three mg of desiccated fucoidan fractions were transferred to FT-IR lens and scanned between $4000-650 \mathrm{~cm}^{-1}$. Measurements were applied to identify and characterize fucoidan fractions in comparison with reference fucoidan. 


\section{g. ${ }^{1} \mathrm{H}-\mathrm{NMR}$}

Few $\mathrm{mg}$ of each samples were dissolved in $\mathrm{D}_{2} \mathrm{O}$ to give $5 \mathrm{~mL}$ of clear solutions. The samples were scanned at $80^{\circ} \mathrm{C}$ by a $400 \mathrm{MHz}$ NMR spectrometer.

\subsubsection{Pharmacological investigations}

a. Anti-coagulant activity

Anti-coagulant studies were performed using the blood coagulation system $\left(\mathrm{BCS}^{\circledR}\right.$ System) which was completely programmed to mix, pre-warm and incubate the reaction contents at $37^{\circ} \mathrm{C}$ in addition to measure the coagulation time in s. The experiments were repeated for three times using platelet-poor plasma isolated from healthy patients' blood, reagents purchased from Siemens Healthcare Diagnostics Products $\mathrm{GmbH}, 0.9 \%$ (w/v) $\mathrm{NaCl}$ as a negative control and $0.005 \mu \mathrm{gL}^{-1}$ as a positive control. Fucoidan fractions were dissolved in an isotonic solution of $0.9 \%(\mathrm{w} / \mathrm{v}) \mathrm{NaCl}$. Fucoidan_1, Fucoidan_6, Fucoidan_M were investigated at a concentration of $0.01 \mathrm{mg} \mathrm{mL}^{-1}$. However, Fucoidan_PDD was investigated in a dose-response manner in concentration ranges of 0.01-0.1 $\mathrm{mg} \mathrm{mL}^{-1}$ in aPPT and 0.001-0.05 $\mathrm{mg} \mathrm{mL}^{-1}$ in TT investigations.

\section{i. $\quad$ Activated Partial Thromboplastin Time (aPTT)}

According to the protocol described by Anderson, et al. [112] with few modifications. With each fucoidan fraction, $0.6 \mathrm{~mL}$ of plasma was mixed with $0.3 \mathrm{~mL}$ of fucoidan solutions. The mixture was then introduced to the blood coagulation system which was programmed to incubate it for $60 \mathrm{~s}$, add $0.5 \mathrm{~mL}$ of pre-warmed Pathrombin ${ }^{\circledR} \mathrm{SL}$ reagent and incubate again for $5 \mathrm{~min}$ at $37^{\circ} \mathrm{C}$. Afterwards, $0.6 \mathrm{~mL}$ of a pre-warmed $0.25 \mathrm{M} \mathrm{CaCl}_{2}$ solution was added and the time for clot formation recorded. In a dose-dependent method, $50 \mu \mathrm{L}$ of each spiked Fucoidan_PDD platelet-poor plasma solution were mixed equally with Pathromtin $\mathrm{SL}^{\circledR}$ reagent, incubated then for $2 \mathrm{~min}, 50 \mu \mathrm{L} \mathrm{CaCl}_{2}$ solution was added and the coagulation time recorded. According to the provider, Pathrombin ${ }^{\circledR}$ SL reagent consisted of silicon dioxide particles $\left(1.2 \mathrm{~g} \mathrm{~L}^{-1}\right)$, plant phospholipids $\left(0.25 \mathrm{~g} \mathrm{~L}^{-1}\right), \mathrm{NaCl}$ and HEPES $(\mathrm{pH} 7.6)$.

\section{ii. $\quad$ Prothrombin Time $(P T)$}

PT was determined following the protocol of Quick [113] with some modifications. The blood coagulation system was also programmed to perform the same procedure mentioned previously in APTT determination, but each plasma and fucoidan solution mixture was incubated for $3 \mathrm{~min}$. Afterwards, $0.6 \mathrm{~mL}$ pre-warmed Dade ${ }^{\circledR}$ Innovin $^{\circledR}$ reagent was added and the time for clot formation 
was observed. Dade ${ }^{\circledR}$ Innovin ${ }^{\circledR}$ reagent was a lyophilized product of recombinant human tissue factor and synthetic phospholipids (thromboplastin), calcium ions, a heparin-neutralizing compound, buffers and stabilizers (BSA), as provided by the producer.

\section{iii. $\quad$ Thrombin Time (TT)}

TT was measured applying the protocol described by Denson and Bonnnar [114] with some modifications. In a ratio of $3: 1,0.6 \mathrm{~mL}$ of plasma was mixed with $0.2 \mathrm{~mL}$ of fucoidan solutions. Each mixture was then incubated for 3 min before the addition of $0.2 \mathrm{~mL}$ pre-warmed BC Thrombin Reagent (BC THROMBIN) and the time for clot formation was recorded. In addition, $50 \mu \mathrm{L}$ of prewarmed Fucoidan-PDD-spiked plasma at different concentrations were mixed with $100 \mu \mathrm{L}$ Thrombin Reagent and the time for the coagulation was measured. BC Thrombin Reagent consisted of lyophilized bovine thrombin $\left(\leq 0.8 \mathrm{IU} \mathrm{mL}^{-1}\right)$ and bovine albumin in HEPES ( $\left.\mathrm{pH} 7.4\right)$, according the product's data sheet.

\section{b. Anti-viral assay}

The anti-viral activity was carried out against a representative of the double stranded DNA (dsDNA) viruses; Herpes Simplex virus-type 1 (HSV-1). The screening assay was based on modified Kleymann and Werling's Tissue Culture Infection Dose 50 (TCID50) protocol for anti-viral candidates [115]. Briefly, a stock solution of $5 \mathrm{mg} \mathrm{mL}^{-1}$ of different fucoidan fractions were dissolved in PBS ( $\mathrm{pH}$ 7.4), while aciclovir as $10 \mathrm{mM}$ in DMSO. The $\mathrm{IC}_{50}$ was determined using a two-fold serial dilution in the range of 0.2 - 100 and $0.054-28 \mu \mathrm{g} \mathrm{mL} \mathrm{m}^{-1}$ for fucoidan solutions and aciclovir, respectively. Each well contained 10,000 cells of Vero B4 cells (50 $\mu \mathrm{L}$ of a solution with $2 \times 10^{5}$ cells $\mathrm{mL}^{-1}$ provided in Roswell Park Memorial Institute medium (RPMI) containing $10 \%$ fetal calf serum (FCS) and penicillin/streptomycin (PS), $50 \mu \mathrm{L}$ pathogen with 5 to $500 \mathrm{CFU}$ of $\mathrm{HSV}-1$ (strain HF ATCC-VR-260), the final volume of each well was then completed to $200 \mu \mathrm{L}$ by the culture medium. Negative controls were performed by mixing $50 \mu \mathrm{L}$ of pathogen, $50 \mu \mathrm{L}$ cell suspensions, and $100 \mu \mathrm{L}$ medium. After the respective incubation period, the wells of the microplate were washed with $200 \mu \mathrm{L}$ PBS and then filled with $200 \mu \mathrm{L} 10 \mu \mathrm{g} \mathrm{mL}^{-1}$ fluorescein diacetate prepared in PBS. Fluorescein diacetate is a non-fluorescent dye and widely used to count viable cells and analyzes their vitality, where viable cells are able to convert the dye enzymatically by a cellular esterase activity releasing the fluorophore from the quenched dye. After $45 \mathrm{~min}$ of incubation at room temperature, RFU was measured using $485 \mathrm{~nm}$ for excitation and $538 \mathrm{~nm}$ for emission. 
c. Anti-microbial activity

With the same principle applied in the anti-viral assay, anti-fungal and anti-bacterial activities of fucoidan fractions were investigated. In anti-fungal activity, Candida albicans, C. tropicalis and $C$. glabrata were chosen as fungal representatives in Vero B4 cell line and amphotericin B was used as a positive control, while $S$. aureus as a Gram +ve and E. coli as a Gram -ve in HeLa and CHO-K1 cell lines were used to detect the anti-bacterial activity of fucoidan fractions. The activity investigation applied ciprofloxacin as a bench marker.

\section{d. Free-Radical scavenging anti-oxidant activity}

According to Mensor [116] and Paul [117], with some modifications, $2 \mathrm{~mL}$ of a freshly prepared methanolic DPPH solution $(0.1 \mathrm{mM})$ was mixed with $0.1 \mathrm{~mL}$ of a serial dilution of aqueous fucoidan fraction solutions in a concentration range of $25-1000 \mu \mathrm{g} \mathrm{mL}^{-1}$. The mixtures were incubated at room temperature in a dark place for $30 \mathrm{~min}$, before absorbance measuring with Uv/Visspectrometer at $517 \mathrm{~nm}$. Ascorbic acid (6.25 - $\left.200 \mu \mathrm{g} \mathrm{mL}^{-1}\right)$ was used as a reference. The percent scavenging activity of each fucoidan fraction in addition to ascorbic acid was calculated applying Eq. 1.

$\%$ Scavenging activity $=100-\left[\left(\mathrm{Abs}_{\text {sample }}-\mathrm{Abs}_{\text {blank }}\right) \times 100\right] / \mathrm{Abs}_{\mathrm{control}} \ldots \ldots \ldots$ (Eq. 1)

Where;

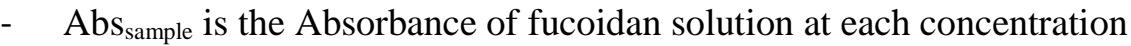

- Absblank is the Absorbance without DPPH

- $\quad \mathrm{Abs}_{\text {control }}$ is the Absorbance without fucoidan

2.2.9. Process scaling-up and application of optimized conditions

Starting with $100 \mathrm{~g}$ of a dried biomass of $F$. vesiculosus, pre-treatment was performed. Then, the resulted pre-treated biomass was extracted as described previously in Procedure A. Procedures for fucoidan purification and recovery were carried out at the optimized conditions by incubation of both TB- and PDD-derivatized beads $(75 \mathrm{mg})$ with $1.5 \mathrm{~mL}$ of the stock solution $\left(2.5 \mathrm{mg} \mathrm{mL}^{-1}\right)$ of Fucoidan_A prepared in $20 \mathrm{mM}$ MES (pH 6). The process was performed in a $2 \mathrm{~mL}$ reaction vessel at room temperature for $40 \mathrm{~h}$ for TB- and $16 \mathrm{~h}$ for PDD-deivatized beads, and placed in an overhead shaker adjusted at $30 \mathrm{rpm}$. The beads were then washed with water and $0.1 \mathrm{M} \mathrm{NaCl}$ in $20 \mathrm{mM} \mathrm{MAB}$ at $\mathrm{pH} 2$, successively for $3 \mathrm{~h}$ and $5 \mathrm{~h}$, respectively. Fucoidan was eluted with $1.5 \mathrm{~mL}$ of $3 \mathrm{M} \mathrm{NaCl}$ 
dissolved in $30 \%$ (v/v) ethanol in a $20 \mathrm{mM}$ MES (pH 6) with vigorous shaking (800 rpm) at $50{ }^{\circ} \mathrm{C}$ for $16 \mathrm{~h}$. To recover fucoidan, a rotary evaporator was utilized to concentrate the eluate and remove ethanol as well $\left(60^{\circ} \mathrm{C}, 90 \mathrm{mbar}\right)$. Concentrated solution was then dialyzed using a dialysis membrane with a $3.5 \mathrm{kDa}$ MWCO. During this procedure, deionized water was changed periodically every $2 \mathrm{~h}$ and conductivity was measured by a conductivity meter set until the resulting solution had the same conductivity value as deionized water. Finally, freezing at $-20^{\circ} \mathrm{C}$ for overnight, then lyophilization in a freeze dryer for $48 \mathrm{~h}$ adjusted at $-20^{\circ} \mathrm{C}$ and 1.03 mbar were carried out to obtain a fluffy white product. 


\subsection{Results and Discussion}

2.3.1. Pre-treatment, extraction and preparation of crude extract

Fucoidan content in F. vesiculosus is affected by seasonal and geographical factors and represents a minor component, in relation to other carbohydrates [79,118,119], as shown in Fig. 14. Therefore, several steps of pre-treatment were carried out to remove extraneous matters (e.g., mannitol, lipids, polyphenols, etc.), which might be co-extracted with fucoidan and interfered afterwards with purification processes $[120,121]$.

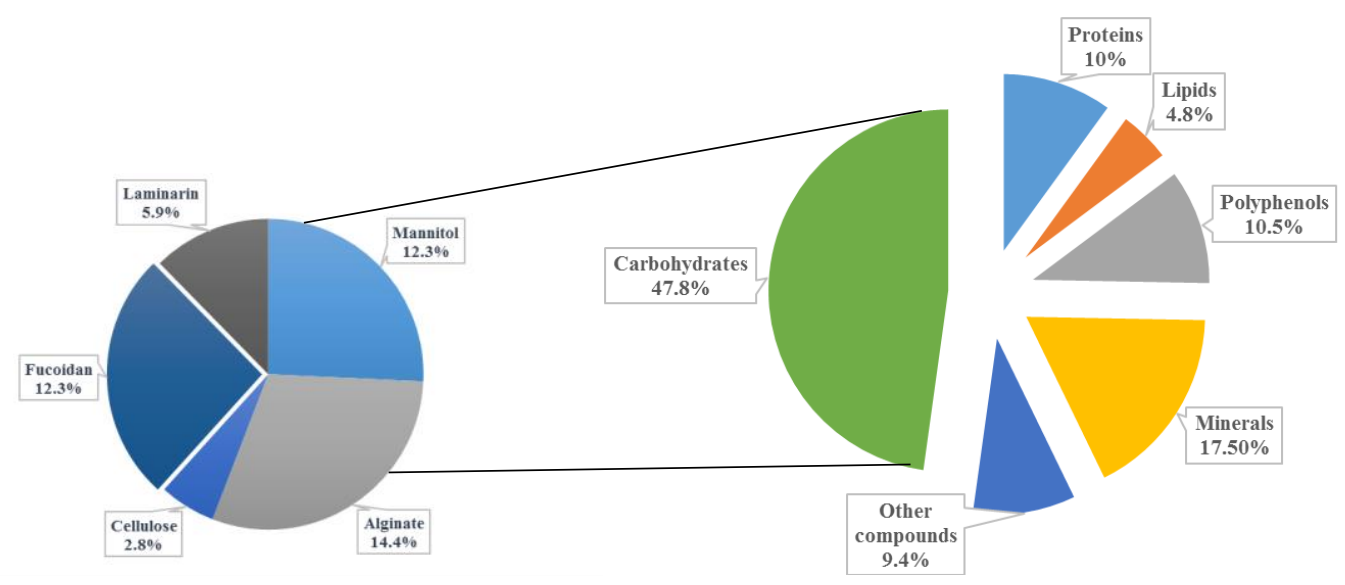

Fig. 14: Average composition of the dried biomass of $\boldsymbol{F}$. vesiculosus [79]

It shows that fucoidan content represents approx. $12 \% \mathrm{w} / \mathrm{w}$ of the dried algal biomass.

Acetone was applied to remove thallus pigments (e.g., chlorophyll), and non-polar fatty acids, while a hexane/isopropanol mixture removed lipids and more polar fatty acids. An $80 \%$ (v/v) ethanol was used to get rid of the major reserve food material mannitol for the algae. An ethanol/water/formaldehyde mixture at $\mathrm{pH} 2$ was used to capture and polymerize polyphenols, which are usually tightly bound to fucoidan in the cell wall and responsible for the brown colour of contaminated fucoidan [122], and finally again with $80 \%(\mathrm{v} / \mathrm{v})$ ethanol for washing and cleaning up the biomass from residual formaldehyde and condensed polyphenol complexes.

An aqueous, acidic conditions and in the presence of calcium ion optimized extraction conditions, and facilitated the precipitation of alginate as $\mathrm{Ca}$ salt using several extraction procedures; Procedure_A, Procedure_B and Procedure_C. Besides, a neutralization step with $(\mathrm{NH} 4)_{2} \mathrm{CO}_{3}$ was carried out in Procedure_A and Procedure_C after extraction to protect fucoidan's glycosidic linkages and sulphate esters from acid hydrolysis [120,121]. Moreover, Procedure_B was performed 
to study the effect of long incubation of fucoidan in acidic condition and possibility to purify fucoidan without the ethanol precipitation step. However, Procedure_C was applied at moderate conditions based on optimized extraction conditions from $F$. vesiculosus previously performed by Hahn, et al. [79]. Different crude fucoidan fractions were precipitated with different volumes of ethanol. In Procedure A, a yield of 2.58 g crude precipitated fucoidan (Fucoidan_A) was obtained, representing $17.5 \%(\mathrm{w} / \mathrm{w})$ from the starting dried algal biomass. However, Procedure_C resulted in $0.37 \mathrm{~g}$ and $0.38 \mathrm{~g}$ as yields from Fucoidan_40\% and Fucoidan_70\%, respectively. The low yield of crude fucoidan obtained from Procedure_C indicated that reflux, high temperature and more acidic conditions were critical factors for better extraction. Moreover, extraction conditions used in Procedure_A confirmed its ability to obtain a higher yield than that stated in the literature.

2.3.2. Characterization of crude fucoidan fractions

Crude fucoidan fractions; Fucoidan_A, Fucoidan_40\% and Fucoidan_70\% before purification protocols were characterized regarding its sugar, fucoidan, fucose and free sulphate contents,

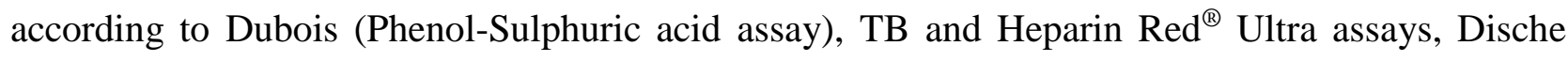
(Cysteine- $\mathrm{H}_{2} \mathrm{SO}_{4}$ assay) and $\mathrm{BaSO}_{4}$ assay, respectively. Results were summarized in Table 4, it shows that Fucoidan_A has the best quality, which had the highest sugar and lowest hydrolyzed free sulphate contents. In addition, its fucoidan and fucose contents were comparable with Fucoidan_70\%.Furthermore, 70\% (v/v) ethanol proved its efficiency to precipitate crude fucoidan with higher fucoidan and fucose contents. However, the low fucoidan content (approx. 70\% w/w) confirmed that there were still co-extracted contaminants with fucoidan, despite of the extensive performed pre-treatment steps, and therefore a further step of purification was needed to obtain an improved product. Fucoidan_A was considered as a candidate to give a high-quality fucoidan and more preferred than the other fractions to perform the further purification process. 
Table 4: Sugar, fucoidan, fucose and sulphate contents in different crude extracted fucoidan fractions from $F$. vesiculosus by Procedure_A and C

\begin{tabular}{|c|c|c|c|c|c|}
\hline \multirow[t]{2}{*}{ Parameter } & \multirow[t]{2}{*}{$\begin{array}{c}\text { Method of } \\
\text { Determination }\end{array}$} & \multirow[t]{2}{*}{$\begin{array}{l}\text { Standard } \\
\text { used }\end{array}$} & \multicolumn{3}{|c|}{$\begin{array}{c}\text { Concentration (g/g crude } \\
\text { fucoidan) }\end{array}$} \\
\hline & & & $\begin{array}{c}\text { Fucoidan_ } \\
\text { A }\end{array}$ & $\begin{array}{c}\text { Fucoidan_ } \\
40 \%\end{array}$ & $\begin{array}{c}\text { Fucoidan_ } \\
\text { 70\% }\end{array}$ \\
\hline Sugar content & $\begin{array}{l}\text { Dubois test (Phenol- } \\
\text { Sulphuric acid assay) }\end{array}$ & D-Glucose & 0.2079 & 0.176 & 0.14 \\
\hline \multirow[t]{2}{*}{$\begin{array}{l}\text { Fucoidan } \\
\text { content }\end{array}$} & TB assay & \multirow{2}{*}{$\begin{array}{c}\text { Fucoidan } \\
\text { (>95\%pure, } \\
\text { Sigma- } \\
\text { Aldrich }^{\circledR} \text { ) }\end{array}$} & 0.731 & 0.0915 & 0.76 \\
\hline & $\begin{array}{c}\text { Heparin Red }{ }^{\circledR} \text { Ultra } \\
\text { assay }\end{array}$ & & 0.69 & n.d* & n.d \\
\hline Fucose content & $\begin{array}{c}\text { Dische assay } \\
\left(\text { Cysteine- } \mathrm{H}_{2} \mathrm{SO}_{4}\right)\end{array}$ & L-Fucose & 0.165 & 0.172 & 0.0654 \\
\hline $\begin{array}{c}\text { Free sulphate } \\
\text { content }\end{array}$ & $\mathrm{BaSO}_{4}$ assay & $\begin{array}{l}\text { Ammonium } \\
\text { sulphate }\end{array}$ & 0.0367 & 0.1559 & 0.0657 \\
\hline
\end{tabular}

The colour developed in Dubois assay is due to the conversion of the sugars into furfural derivatives by a dehydration reaction with sulphuric acid. The furfural product is condensed with phenol to a coloured compound, which can be detected colorimetry at $490 \mathrm{~nm}$. Colour formation is stable for several hours and proportional to the amount of the sugar present. Its detection limit is $7 \mu \mathrm{g} \mathrm{L}^{-1}$ [105].

In addition, the Dische assay is a specific-oxidation reaction for methylpentoses like fucose. Cysteine combines with the different breakdown compounds of sugars yielding products with different absorption spectra. Therefore, relative absorbance should be measured at two wavelengths to eliminate possible interferences from other sugars. The detection limit of this test is $10 \mu \mathrm{g} \mathrm{L}^{-1}$ [105].

Moreover, TB assay was considered a specific test to determine fucoidan content. The test is based on a metachromatic change of TB due to a charge-transfer complex formation when reacted with polyanionic polymers in acidic or slightly acidic $\mathrm{pH}$, such as fucoidan [98]. The assay was preferred to be performed in a highly acidic $\mathrm{pH}(\mathrm{pH} 1)$ to ensure the specificity and sensitivity for sulphated compounds. At $\mathrm{pH} \mathrm{1,} \mathrm{only} \mathrm{sulphate} \mathrm{groups} \mathrm{of} \mathrm{fucoidan} \mathrm{could} \mathrm{ionize,} \mathrm{because} \mathrm{of} \mathrm{their} \mathrm{lower} p \mathrm{Ka}$ value than other carboxylic and phosphate groups from alginate and nucleic acids, respectively. Moreover, in acidic medium amino groups of thiazine dyes were also protonated and could form a donor-acceptor complex specifically with fucoidan. 
In addition to thiazine dyes, Heparin $\operatorname{Red}^{\circledR}$ (Redprobes UG) proved its effectiveness in the detection of heparin in plasma [123] and urine [124] and, more recently, for the quantification of fucoidan in spiked plasma [106]. The reaction is based on the formation of electrostatically-driven aggregates with polyanionic sulphated polysaccharides, such as heparin and fucoidan followed by fluorescent quenching, as shown in Fig 15. To assure test selectivity, Na-alginate samples were analyzed separately, which showed no affinity and fluorescence quenching activity toward Heparin Red ${ }^{\circledR}$ probes.

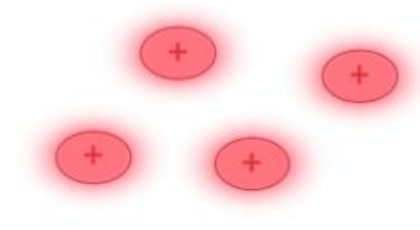

Fluorescent, polycationic probe molecules (e.g., Heparin Red)
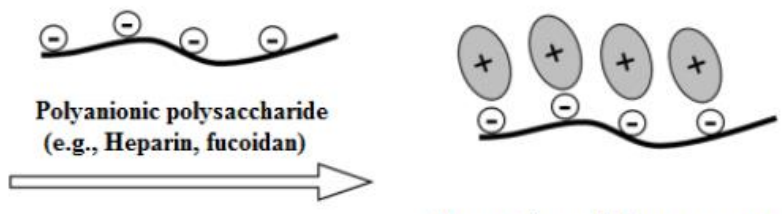

Aggregation and fluorescence quenching

Fig. 15: Representation of the polyanionic polysachharide reaction with fluorescent Heparin $\operatorname{Red}^{\circledR}$

The reaction resulted in formation of electrostatically aggregates and followed by fluorescence quenching (modified according to [125]).

Furthermore, reaction of free sulphate ions with barium ions produced $\mathrm{BaSO}_{4}$ as a precipitate. The solution turbidity could be measured at a $\lambda$ of $600 \mathrm{~nm}$. This principle was applied to quantify free sulphate content in crude fucoidan fractions. Lower free sulphate content is advantageous and indicated lower percent of decomposed or hydrolysed sulphate ester groups during extraction step.

2.3.3. Application and optimization of fucoidan purification

Fucoidan_A was used in application and optimization of purification of Hahn's protocol [108] due to its higher production yield and better quality as discussed previously in section 2.3.2. Investigated conditions were classified to be either during fucoidan's adsorption phase or elution phase. In addition, several fractions of purified fucoidan were isolated to be characterized afterwards to reach the optimum purification conditions regarding physico-chemical, pharmacological properties in addition to the degree of purity. 


\subsubsection{Adsorption or incubation phase}

a. Kinetic, adsorption $\mathrm{pH}$ and type of thiazine dye

Fucoidan adsorption (\%) by immobilized $2 \mathrm{mM}$ TB, TA and mixed TA and TB were compared at pH 1 and pH 6. As shown in Fig. 16, results revealed that fucoidan required long incubation periods to be captured significantly confirming the results obtained previously from kinetic and sorption isotherm studies by Hahn [108]. In addition, capture (\%) was higher at highly acidic pH 1 than slightly acidic pH 6 conditions. Therefore, it could be concluded that at $\mathrm{pH} 1$, a partial acid hydrolysis of fucoidan occurred and the diffusion rates (rate limiting step) of the lower molecular weight fucoidan [94] were possibly improved making the adsorption easier and faster through immobilized dyes found on the beads surface and in pores. In addition, the highly acidic $\mathrm{pH}$ may help more sulphate ester groups to be ionized which interacted proportoionally with TB. Furthermore, similar adsorption $(\%)$ by mixed dyes proved that both beads did not enhance or compete with each other during fucoidan capturing process. 

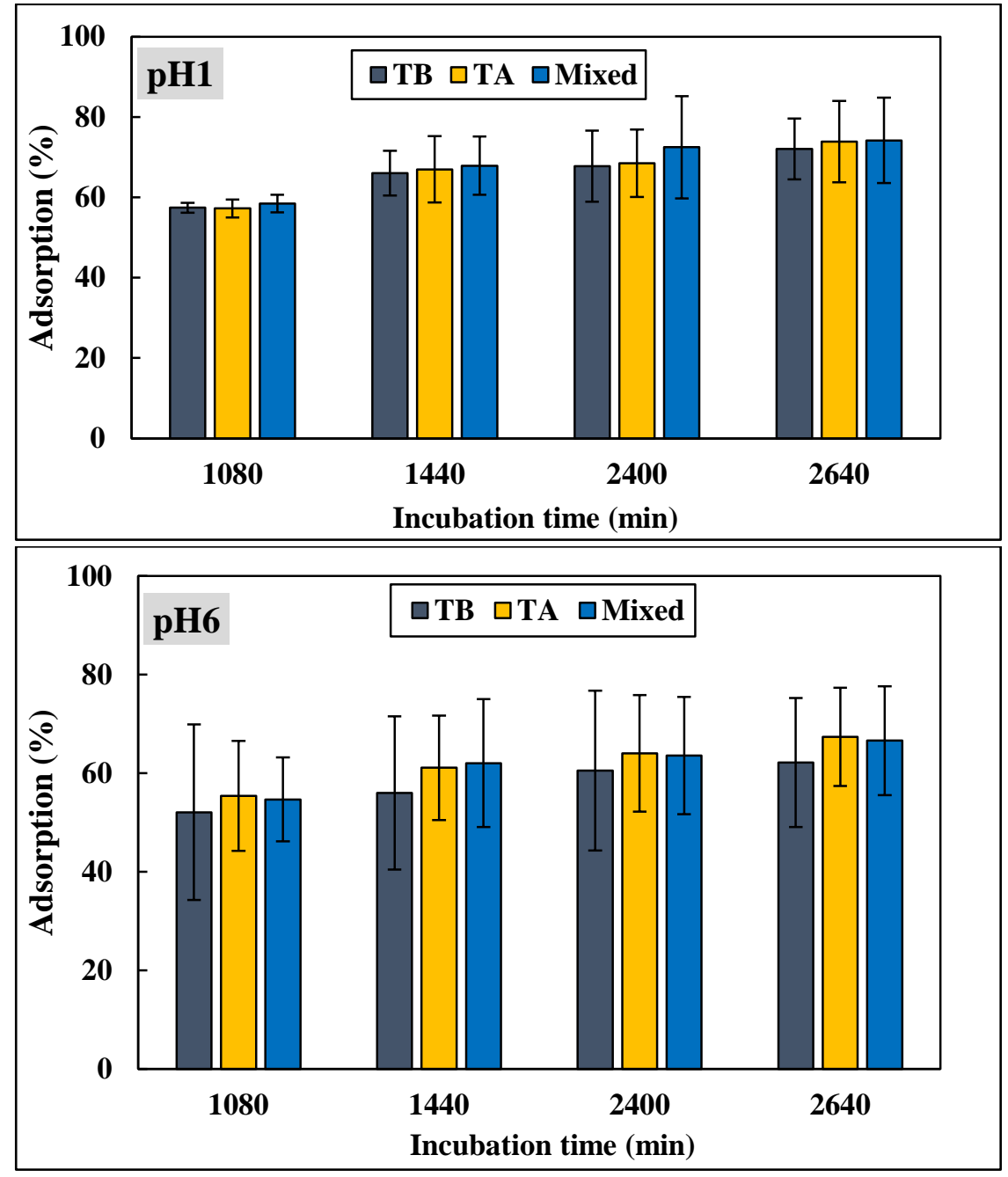

Fig. 16: Adsorption (\%) of fucoidan by immobilized $2 \mathrm{mM} \mathrm{TB}$, TA and mixed dyes at pH 1 and pH 6 The adsorption (\%) was determined after incubation of $1.5 \mathrm{~mL} 2.5 \mathrm{mg} \mathrm{mL}^{-1}$ of Fucoidan_A at 1080, 1440, 2400 and $2640 \mathrm{~min}$ with $50 \mathrm{mg}$ of derivatized beads at room temperature.

b. Molarity of immobilized dye

As a trial to increase the availability of dyes for fucoidan, and therefore a higher percent of captured fucoidan, immobilization of higher concentrations of dyes; 4 and $6 \mathrm{mM}$ of thiazine dyes, were applied. Results of adsorption at $\mathrm{pH} 1$ showed that, not only the percent of captured fucoidan was not significantly affected by increasing the dye molarity, as shown in Fig. 17, but also dyes leaching was higher than immobilized $2 \mathrm{mM}$. Because of these reasons, $2 \mathrm{mM}$ immobilized TB was preferred in the downstream process for purified fucoidan production. 


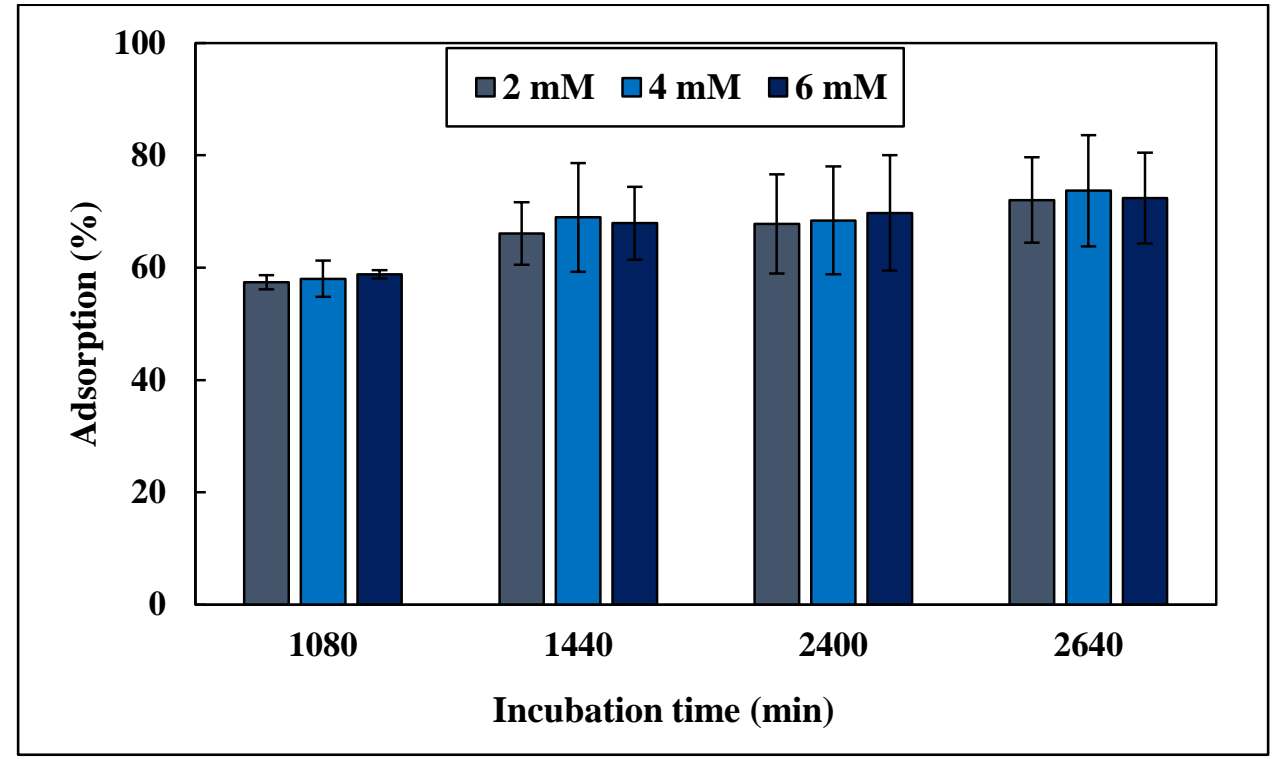

Fig. 17: Comparison between adsorption (\%) of fucoidan by immobilized 2, 4 and $6 \mathrm{mM}$ TB at pH 1

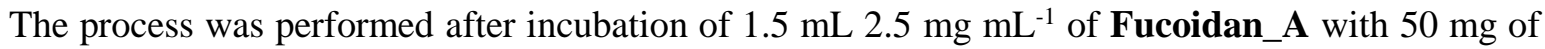
derivatized beads at room temperature. Samples from supernatant were taken at 1080, 1440, 2400 and $2640 \mathrm{~min}$ of incubation and analyzed to determine adsorption rates.

c. Incubation time

Adsorption of fucoidan is a time-dependent process and required at least $30 \mathrm{~h}$ of incubation to attain the equilibrium between beads and available fucoidan [94], and therefore an extended incubation period was studied for 60 h. As shown in Fig. 18, captured fucoidan (\%) was increased by time at both $\mathrm{pH}$, especially at $\mathrm{pH} 1$, but the possibility of a non-specific acid hydrolysis for fucoidan may be occurred, and therefore affected the native molecular weight of fucoidan at these conditions. 


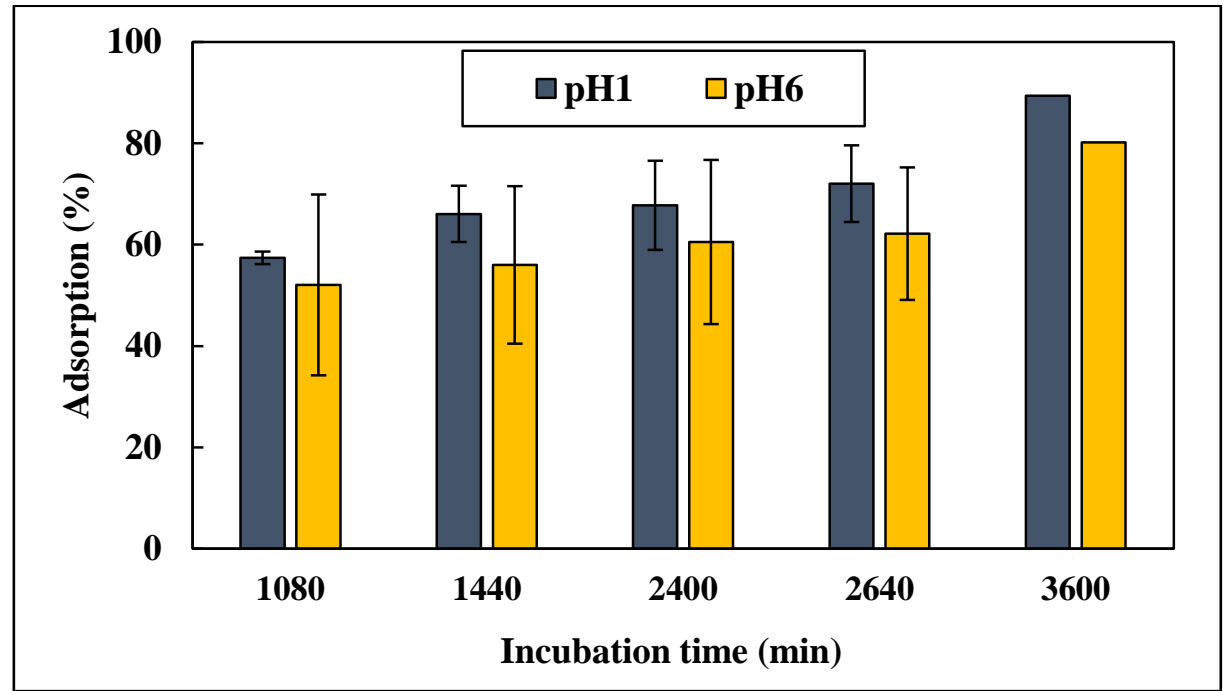

Fig. 18: Adsorption (\%) of fucoidan by $2 \mathrm{mM}$ immobilized TB for $60 \mathrm{~h}$ of incubation at pH 1 and $\mathrm{pH} 6$

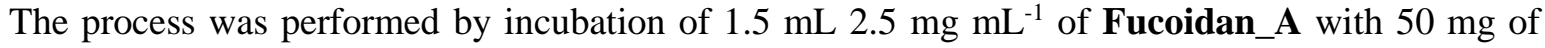
derivatized beads at room temperature for $60 \mathrm{~h}$ (3600 min). Samples from supernatant were taken at 1080, 1440, 2400, 2640 and 3600 min of incubation and analyzed to determine adsorption rates.

d. Quantity of derivatized beads

As shown in Fig. 19, incubation of crude fucoidan at pH 1 and 6 with $75 \mathrm{mg}$ of TB-derivatized beads succeeded to increase adsorption (\%) to $99.5 \%$ and $83.8 \%$, respectively after $44 \mathrm{~h}$ of incubation. This was another proof that the non-completed adsorption, long incubation periods and difficultly to reach the equilibrium state were related to the the beads porosity and the slower and variable diffusion rates of fucoidan polymers. 


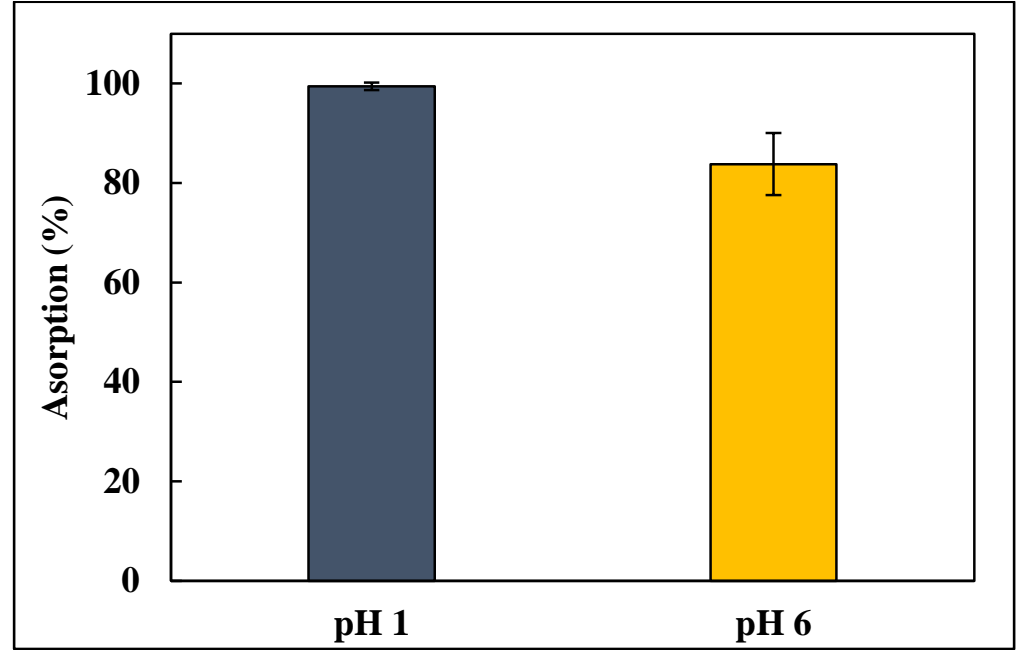

Fig. 19: Adsorption (\%) of fucoidan from Fucoidan_A by 75 mg of $2 \mathrm{mM}$ immobilized TB at pH 1 and pH 6

The process was performed by incubation of $1.5 \mathrm{~mL} 2.5 \mathrm{mg} \mathrm{mL}^{-1}$ of Fucoidan_A at room temperature for $44 \mathrm{~h}$ in a $2 \mathrm{~mL}$ reaction vessel.

e. Effect of multiple use of immobilized beads

TB-derivatized beads were used for several cycles to adsorb fucoidan from raw synthetic extract efficiently [94]. Similarily, immobilized TB showed reproducible and comparable fucoidan adsorption (\%) from the crude extract of $F$. vesiculosus with multiple use in two cycles, as shown in Fig. 20. This factor confirmed also that elution step regenerateed the beads' function without the need to an additional regeneration step as in IEX process. 


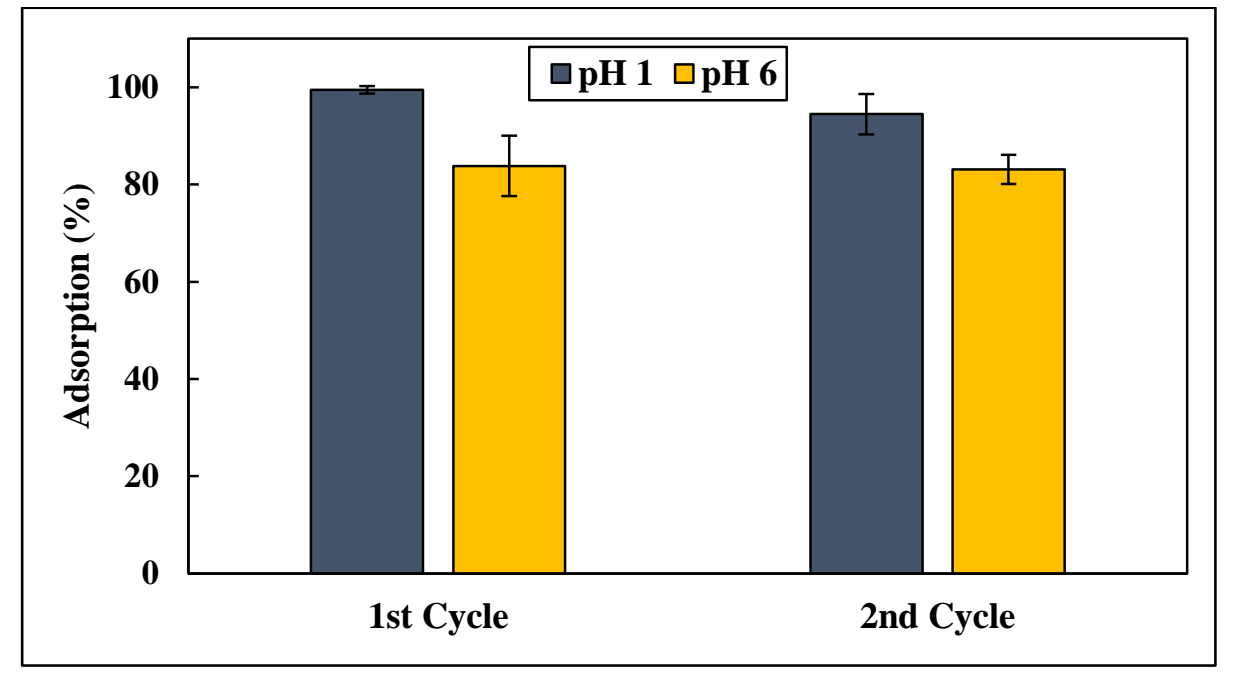

Fig. 20: Adsorbed fucoidan (\%) by $75 \mathrm{mg}$ of immobilized TB for two cycles at pH 1 and pH 6

The process was performed by incubation of $1.5 \mathrm{~mL} 2.5 \mathrm{mg} \mathrm{mL}^{-1}$ of Fucoidan_A at room temperature for $44 \mathrm{~h}$ in a $2 \mathrm{~mL}$ reaction vessel.

\subsubsection{Elution phase}

For elution and effective break down of the charge-transfer complex formed between the immobilized TB and the adsorbed fucoidan, high molar concentration of $\mathrm{NaCl}$, ethanol and heating were required. Ethanol was used to enhance the hydrophobic interaction between the stacked dye molecules. In addition, high temperatures e.g., $50^{\circ} \mathrm{C}$ provided the necessary activation energy for desorption and $\mathrm{NaCl}$ for suppression of ionic interactions between the dye and the sulphated polysaccharide [94]. Only the effect of $\mathrm{NaCl}$ molarity on elution of fucoidan (\%) was studied.

As demonstrated in Fig. 21, increasing of $\mathrm{NaCl}$ molarity in eluent resulted in increasing of eluted fucoidan (\%). Molarity of $\mathrm{NaCl}$ resulted in obtaining different fractions of fucoidan with different molecular weights (discussed later). The process was tested in adsorbed fucoidan at $\mathrm{pH} 1$ and showed that $3 \mathrm{M} \mathrm{NaCl}$ was the best and succeeded to elute $70.3 \%$ in average of adsorbed fucoidan. However, non-completed elution could be related to the difficulty of elution of fucoidan present in the beads pores, and therefore further optimization were still required. In addition, leaching of the immobilized dye might be possible which reacted with some the free eluted fucoidan molecules and total eluted fucoidan could not be determined exactly by the TB assay as a result. Non-eluted or strongly bound fucoidan affected negatively on the beads' efficiency with multiple uses as a result, as shown previously in Fig. 20. In addition, leached dye from derivatized beads at the elution conditions decreased the number of immobilized molecules with each cycle. 


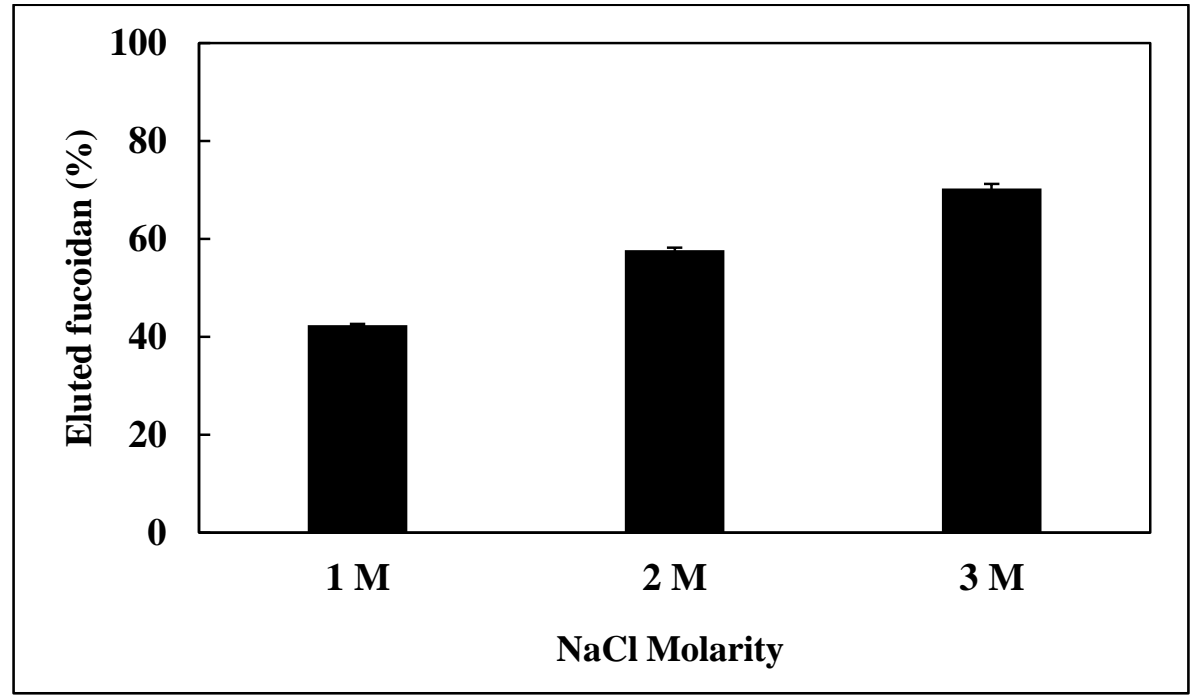

Fig. 21: Effect of $\mathrm{NaCl}$ molarity in eluent on eluted fucoidan (\%)

Elution (\%) was increased by increasing $\mathrm{NaCl}$ molarity and performed for $16 \mathrm{~h}$ in a thermoshaker at a temperature of $50^{\circ} \mathrm{C}$ and vigorous shaking at $800 \mathrm{rpm}$.

Results obtained from investigated factors revealed that neither a completed adsorption of fucoidan nor the maximum loading capacity of immobilized dye were not achieved till $60 \mathrm{~h}$ of incubation at both $\mathrm{pH}$ values using $50 \mathrm{mg}$ of beads with $2.625 \mathrm{mg}$ of fucoidan. However, the reaction between TB and fucoidan occurred immediately. The previously-studied sorption isotherm concluded that $72 \mathrm{~h}$ were required to reach the maximum capacity of beads $\left(127.7 \mathrm{mg} \mathrm{g}^{-1}\right)$ [94]. This might be explained by several reasons including the presence of co-extracted contaminants which delayed fucoidan adsorption or competed for adsorption sites, differences in polymers chain lengths and molecular weights which led to different diffusion rates and beads porosity as well. Moreover, amino groups in thiazine dyes were a limiting factor; i.e., one group per each dye molecule after immobilization, as demonstrated in Fig. 22. Based on these arguments, further optimization steps should be performed to overcome these disadvantages. 


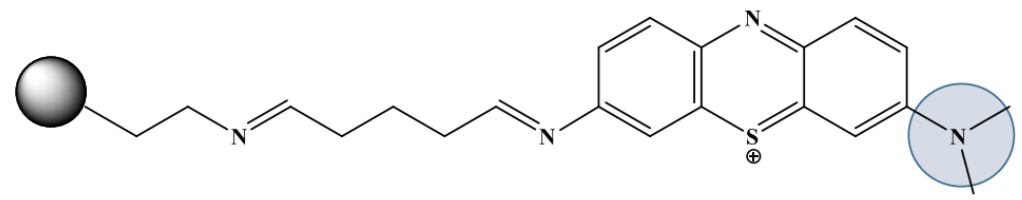

Immobilized TB

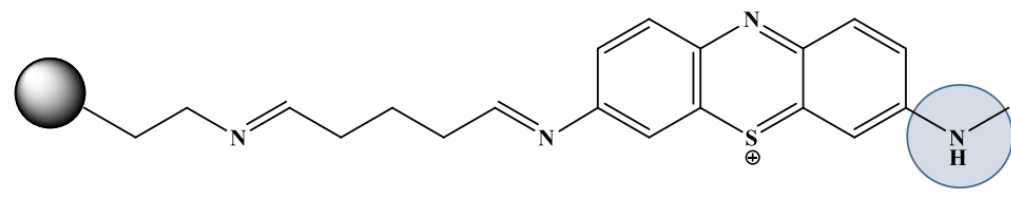

Immobilized TA

Fig. 22: Immobilized TB and TA on Sepabeads ${ }^{\circledR}$ EC-EA

After immobilization, they showed only one available amino group which could be ionized to capture fucoidan.

\subsubsection{Application of PDD for fucoidan purification}

Following the high affinity and sensitivity of Heparin $\operatorname{Red}^{\circledR}$ to polyanionic polysaccharides [106], a fluorescent structurally-related compound to Heparin $\mathrm{Red}^{\circledR}$; polycationic perylene diimide derivative or PDD ( $N, N^{\prime}$-Bis-(1-amino-4,9-diaza dodecyl)-1,7-di bromo perylene-3,4,9,10tetracarboxylic acid diimide), as shown in Fig. 23, was synthesized and immobilized successfully.

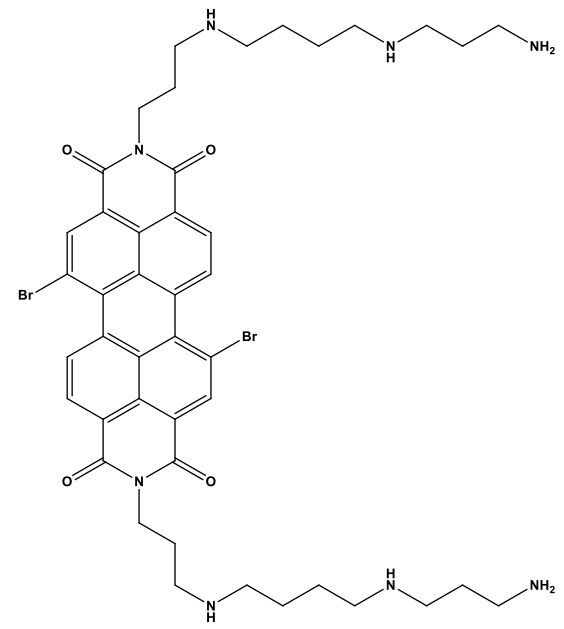

(a)

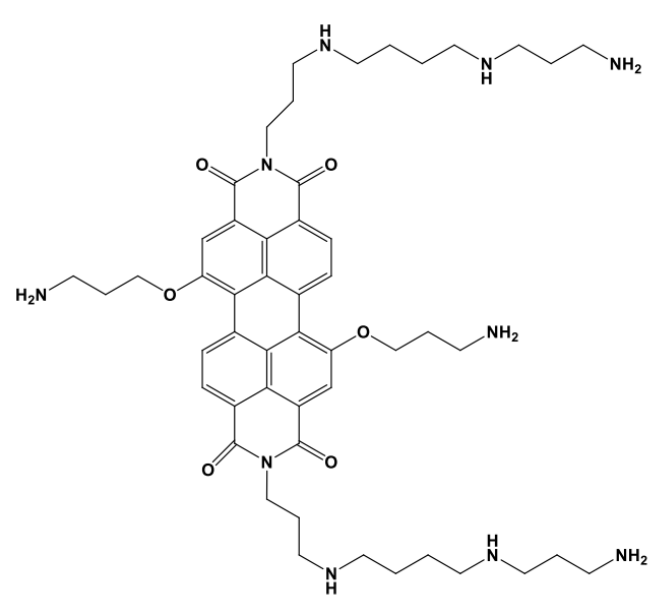

(b)

Fig. 23: Molecular structure of a) perylene diimide derivative ( $N, N^{\prime}$-Bis-(1-amino-4,9-diaza dodecyl)1,7-di bromo perylen-3,4:9,10-tetracarboxylic acid diimide, and b) Heparin Red $^{\circledR}$ PDD shows different primary and secondary amino groups available for immobilization on aminoderivatized beads through a glutaraldehyde bridge as well as formation of a charge transfer-like aggregate with fucoidan in acidic or neutral $\mathrm{pH}$. 
PDD showed a lot of advantages in comparison with thiazine dyes. These advantages include five adsorption amino groups, as shown in Fig. 24, in addition to the wide distance between beads and amino groups which may ease their function to hook the huge and slower fucoidan macromolecules. Moreover, availability of adsorption sites on beads surface overcame the problem of beads porosity.

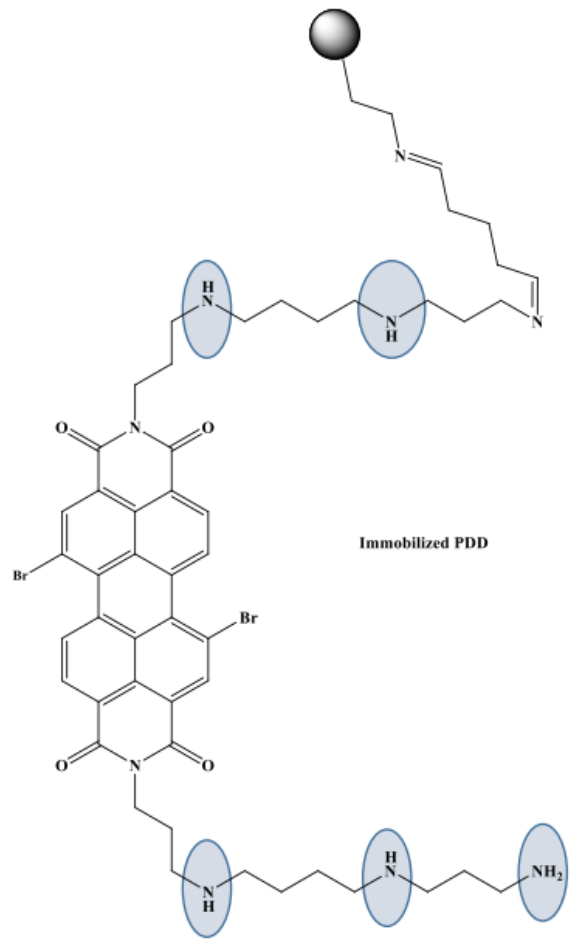

Fig. 24: Immobilized PDD on Sepabeads ${ }^{\circledR}$ EC-EA

It shows five amino groups for fucoidan binding. The figure is based on a 1:1 ratio of immobilization between beads and PDD molecules.

\subsubsection{Adsorption kinetic in crude fucoidan}

As shown in Fig. 25, application of immobilized PDD at $\mathrm{pH} 6$ proved its efficiency to capture fucoidan from crude extract and improved purification process drastically. More than $80 \%(83 \%)$ of available fucoidan was captured in $960 \mathrm{~min}$, compared with $83 \%$ in 2640 min with immobilized TB incubated at the same conditions, as shown previously in Fig. 19. 


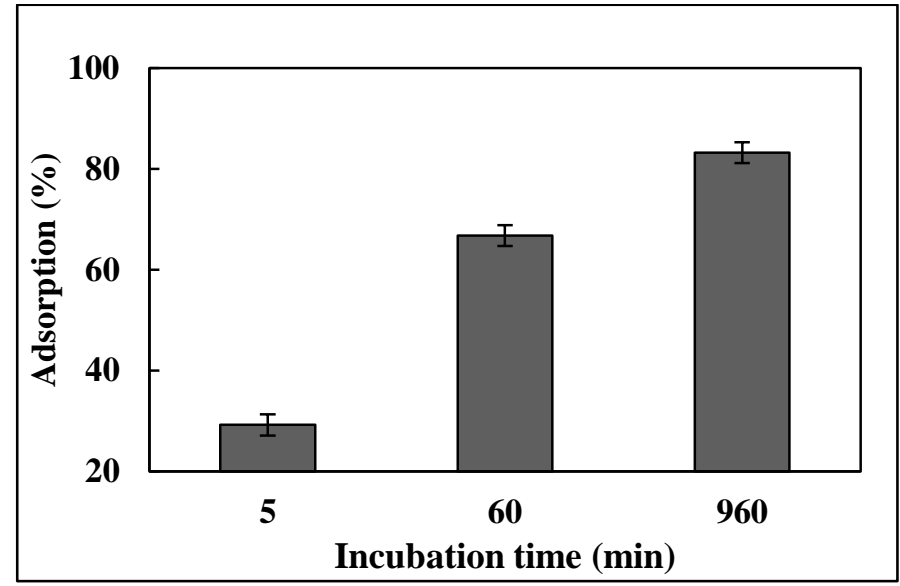

Fig. 25: Time dependence adsorption (\%) of fucoidan from Fucoidan_A by immobilized PDD

The process was performed using $75 \mathrm{mg}$ derivatized beads with a $2.5 \mathrm{mg} \mathrm{mL}^{-1}$ solution of Fucoidan_A in MES (pH 6) at room temperature and a $2 \mathrm{~mL}$ reaction vessel.

\subsubsection{Multiple use of immobilized PDD}

Moreover, PDD-derivatized beads were recycled like immobilized TB without effect on beads efficiency, as shown in Fig. 26. Interestingly, it was observed that the beads were able to adsorb much fucoidan (\%) in the $2^{\text {nd }}$ and $3^{\text {rd }}$ cycles, which were constant, than the $1^{\text {st }}$ cycle. This observation might be due to a non-optimized PDD solution used in immobilization step. Much concentrated PDD solution saturated the beads and the additional molecules, despite of the several washing steps, bound to fucoidan and inhibited its adsorption in the $1^{\text {st }}$ cycle.

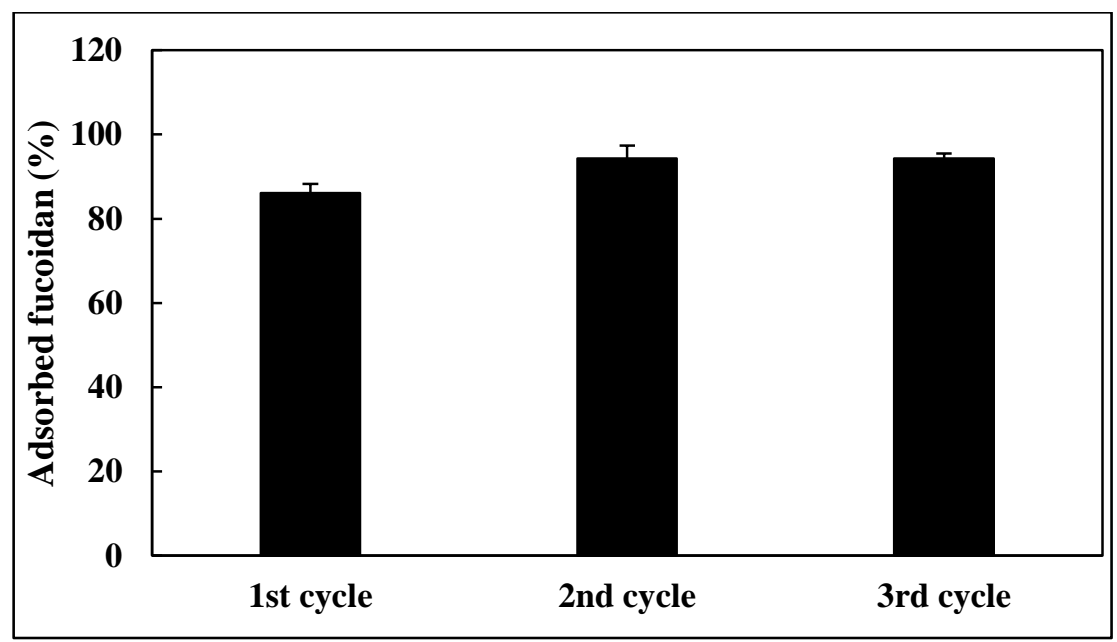

Fig. 26: Multiple use of PDD-derivatized beads for three cycles

Process was performed using $75 \mathrm{mg}$ beads with $2.5 \mathrm{mg} \mathrm{mL}^{-1}$ of Fucoidan_A solution in MES (pH 6) which incubated for $18 \mathrm{~h}$ (1080 $\mathrm{min})$ in a $2 \mathrm{~mL}$ reaction vessel. 


\subsubsection{FPLC automated purification process}

Preliminary results of batch process were a motivation to establish a protocol using FPLC. This could help automation, improve downstream processing, further minimize the time required for the process, and overcome the disadvantages regarding manual solvents exchange during the different purification phases. Furthermore, this process might help in an industrial-scale fucoidan production. The new protocol was developed to imitate batch process with its four phases and performed in only $150 \mathrm{~min}$. A sample of $50 \mathrm{mg} \mathrm{mL}^{-1}$ was prepared, which was less than the maximum loading capacity of the column material, due to a crude fucoidan solubility problem. Concentrations more than 50 mg. $\mathrm{mL}^{-1}$ resulted in a turbid solution. First, $20 \mathrm{mM}$ MES buffer (pH 6) was used for column conditioning. Then, a $50 \mathrm{mg} \mathrm{mL}^{-1}$ sample in MES was injected in $1 \mathrm{~mL}$ loop, followed by buffer again at a slower flow rate to give adequate time for fucoidan adsorption by derivatized beads. Deionized water and $0.1 \mathrm{M} \mathrm{NaCl}$ in $\mathrm{MAB}(\mathrm{pH} 2)$ were used afterwards for washing to remove the non-specific bound molecules. Investigation of flow through and washing collected fractions by TB assay confirmed a complete adsorption of fucoidan.With elution by $3 \mathrm{M} \mathrm{NaCl}$ in $30 \%$ ethanol, fucoidan started to leave its complex easily when conductivity and $\mathrm{pH}$ were increased to approx. 95 $\mu \mathrm{S} / \mathrm{cm}$ and slightly acidic $\mathrm{pH} 6$ (see Appendix), respectively. A similar elution pattern of fucoidan, as shown in Fig. 27, was observed in two cycles.

Fucoidan was observed to be eluted and reached its maximum concentration earlier with the $2^{\text {nd }}$ application of the derivatized beads. Fractions (6-20) from the $1^{\text {st }}$ and $2^{\text {nd }}$ cycles were pooled $(30 \mathrm{~mL}$ each) to give yields of Fucoidan_PDD_1 and Fucoidan_PDD_2 of 16.9 and $16.3 \mathrm{mg}$, respectively. 


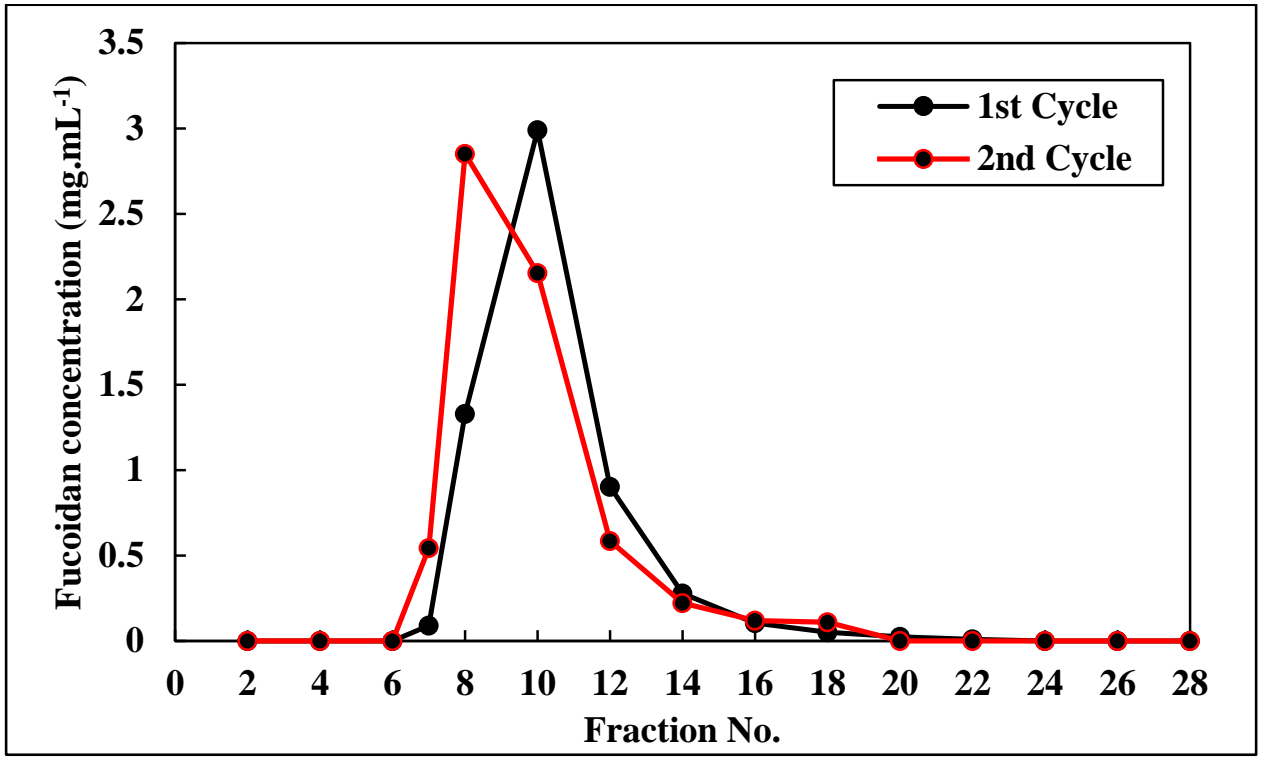

Fig. 27: Fucoidan elution pattern from FPLC column in two successive cycles

Similar pattern of fucoidan elution was observed in the two cycles of fucoifan purification by FPLC. Fucoidan concentrations were determined in column eluates by TB assay.

If the fucoidan content of the injected sample $\left(50 \mathrm{mg} \mathrm{mL}^{-1}\right)$ was considered, only $68 \%$ (34 $\left.\mathrm{mg}\right)$ represented fucoidan. This means that the column could elute only $50 \%$ of the introduced fucoidan. The low elution (\%) might be due to the absence of heating and vigorous shaking applied in batch process.

2.3.5. Physico-chemical characterization of purified fucoidan fractions After freeze-drying, white and hygroscopic fluffy powders were obtained as in Fucoidan_1, Fucoidan_6 and Fucoidan_PDD, while Fucoidan_M was white to brownish colour. All of them were soluble in water, sparingly soluble in DMSO, and insoluble in ethanol.

\subsubsection{Elemental Analysis (CHNS Analysis)}

As shown in Table 5, the presence of traces of nitrogen content $\mathrm{N}(\%)$ in all fucoidan types, in contrast with reference fucoidan, suggested the successful removal of a high percent of proteinaceous compounds. These traces were possibly due to the presence of some amino sugars $[43,126]$. Fucoidan_PDD demonstrated the lowest N $(\%) ; 0.2 \%(\mathrm{~m} / \mathrm{m})$ compared with the other fractions. In contrast, Fucoidan_M showed the highest S\%; 12.11\% $(\mathrm{m} / \mathrm{m})$, which is critical for some biological activities (e.g., anti-viral activity). Based on CHNS analysis, number of sulphate 
ester group per sugar unit and degree of sulphation were calculated using the ratio between C (\%) and S (\%) contents, as shown in Eq. 2 and Eq. 3, respectively. Fucoidan fractions' degree of sulphation were consistent and interesting compared with literature's values, especially with Fucoidan_6 and Fucoidan_PDD [127].

$$
\begin{gathered}
\text { No. of sulphate group per sugar unit }=\left[\frac{\frac{C(\%)}{12}}{\frac{S(\%)}{32}}\right] / 6 \ldots \ldots \ldots \ldots \ldots \text { (Eq. 2) } \\
\text { Degree of sulphation }=1 / \text { No. of sulphate group per sugar unit } \ldots \ldots \ldots . .
\end{gathered}
$$

Where; 12 is the atomic weight of Carbon, 6 is the number of Carbon atoms in the sugar monomer, and 32 is the atomic mass of Sulphur.

Table 5: Elemental analysis (CHNS) and degree of sulphation results of different fucoidan fractions

The results were compared with commercially-available analogue purchased from SigmaAldrich $^{\circledR}$ in addition to calculated degree of sulphation.

\begin{tabular}{|c|c|c|c|c|c|}
\hline Sample & $\mathbf{N}(\boldsymbol{\%})$ & $\mathbf{C}(\boldsymbol{\%})$ & $\mathbf{H}(\boldsymbol{\%})$ & $\mathbf{S}(\boldsymbol{\%})$ & $\begin{array}{c}\text { Degree of } \\
\text { sulphation }\end{array}$ \\
\hline Reference fucoidan & n.d.* & 24.78 & 4.42 & 8.89 & 0.8 \\
\hline Fucoidan_1 & 0.3 & 24.14 & 4.43 & 11.18 & 1.09 \\
\hline Fucoidan_6 & 0.34 & 26.12 & 4.63 & 9.83 & 0.84 \\
\hline Fucoidan_PDD & 0.22 & 25.35 & 4.51 & 8.45 & 0.75 \\
\hline Fucoidan_PDD_1 & 0.3 & 25.77 & 4.57 & 8.78 & 0.766 \\
\hline Fucoidan_PDD_2 & 0.29 & 25.95 & 4.54 & 8.78 & 0.761 \\
\hline Fucoidan_M & 0.26 & 23.31 & 4.28 & 12.11 & 1.16 \\
\hline $\begin{array}{c}\text { Crude fucoidan } \\
\text { (Fucoidan_A) }\end{array}$ & 0.59 & 22.89 & 4.54 & 6.88 & 0.67 \\
\hline
\end{tabular}

* not detected.

Reference fucoidan ( $>95 \%$ pure isolated from F. vesiculosus) was purchased from Sigma-Aldrich ${ }^{\circledR}$; Fucoidan_1 was purified by immobilized TB from crude fucoidan at $\mathrm{pH} 1$; Fucoidan_6 was purified by immobilized TB from crude fucoidan at $\mathrm{pH} 6$; Fucoidan_PDD was purified by immobilized PDD from crude fucoidan at $\mathrm{pH}$ 6; Fucoidan_PDD_1 was purified using a FPLC protocol from crude fucoidan at $\mathrm{pH} 6\left(1^{\text {st }}\right.$ cycle $)$; Fucoidan_PDD_1 was purified using a FPLC protocol from crude fucoidan at $\mathrm{pH} 6$ ( $2^{\text {nd }}$ cycle $)$; Fucoidan_M was purified from MAB crude extract at $\mathrm{pH} 1$ without crude fucoidan precipitation. 
The higher degree of sulphation, especially in Fucoidan_1 and Fucoidan_M, were due to lower $\mathrm{C}(\%)$ to $\mathrm{S}(\%)$ ration which was affected by highly acidic $\mathrm{pH}$ and resulted in polymer hydrolysis, as will be discussed in the following section 2.3.5.2.

\subsubsection{Molecular weight parameters}

For ideal characterization of any polymer, three different parameters should be calculated, which are Mw, Mn and Mp. Mn indicates the number of molecules in a sample of a given weight, while Mw takes into account the molecular weight of chains contributing to the molecular weight average [128] and Mp determines the polymer molecular weight at the top of peak or the most prominent molecular weight. In addition, the PDI measures the broadness of molecular weight distribution of polymers; the larger the PDI, the broader the molecular weight. According to Agilent Technologies [128], the following equations (Eq. 4 - 6) were applied.

$$
\begin{aligned}
& M n=\frac{\sum N_{i} M_{i}}{\sum N_{i}} \quad \ldots \ldots \ldots \ldots \ldots . . \quad\left(\text { Eq. 4) } \quad M w=\frac{\sum N_{i} M_{i}^{2}}{\sum N_{i} M_{i}} \ldots \ldots \ldots \ldots \ldots\right. \text { (Eq. 5) } \\
& P D I=\frac{M w}{M n} \ldots \ldots \ldots \ldots \ldots \ldots . .(\text { Eq. 6) }
\end{aligned}
$$

Where; $\boldsymbol{M}_{\boldsymbol{i}}$ is the molecular weight of a chain, and $\boldsymbol{N}_{\boldsymbol{i}}$ is the number of chains of that molecular weight.

Natural polymers like proteins and polysaccharides are monodisperse and therefore have PDI near to 1. As demonstrated in Table 6a, purification processes either by immobilized TB or PDD improved molecular weight parameters and its quality as well. Fucoidan_1 and Fucoidan_M showed the lowest- measured Mw and this proved that long incubation periods in acidic conditions during adsorption phase had a strong hydrolytic effect on fucoidan polymer, despite of higher adsorption rates. Moreover, low molecular weight obtained from FPLC method suggested that elution conditions were only able to elute low molecular weight fucoidan (LMWF).

Not only \% eluted fucoidan, but also molecular weight averages of eluted fucoidan fractions were affected by the $\mathrm{NaCl}$ molarity. Table $\mathbf{6 b}$ shows that molecular weight of fucoidan purified at the same conditions could be sub-fractionated by gradient elution using different molarities of $\mathrm{NaCl}$. The higher $\mathrm{NaCl}$ molarity applied, the more fucoidan molecular weight is obtained. 
Table 6a: Molecular weight parameters and polydispersity index (PDI) of different purified fucoidan fractions.

The fucoidan fractions were compared with reference fucoidan purchased from Sigma-Aldrich ${ }^{\circledR}$ and crude fucoidan before purification

\begin{tabular}{|c|c|c|c|c|}
\hline Fucoidan type & $\mathbf{M w x 1 0}^{\mathbf{4}}$ & $\mathbf{M n x 1 0}^{\mathbf{4}}$ & $\mathbf{M p x 1 0}^{\mathbf{4}}$ & PDI \\
\hline Reference fucoidan & 9.5 & 4.2 & 5.1 & 2.2 \\
\hline Fucoidan_1 & 5.8 & 3.9 & 3.3 & 1.4 \\
\hline Fucoidan_6 & 9.8 & 5.4 & 5.7 & 1.8 \\
\hline Fucoidan_PDD & 8.6 & 4.2 & 3.2 & 2.04 \\
\hline Fucoidan_PDD_1 & 5.8 & 2.5 & 3.4 & 2.3 \\
\hline Fucoidan_PDD_2 & 6.6 & 3.98 & 4.02 & 1.66 \\
\hline Fucoidan_M & 4.8 & 1.8 & 2.6 & 2.6 \\
\hline Crude fucoidan & 6.6 & 3.1 & 4.2 & 2.1 \\
\hline
\end{tabular}

Mw: weight average molecular weight, Mn: Number average molecular weight, and Mp: peak molecular weight

Table 6b: Molecular weight parameters of fractions obtained by different $\mathrm{NaCl}$ molarity Fractionation was performed by applying different 1,2 and $3 \mathrm{M} \mathrm{NaCl}$ in eluent.

\begin{tabular}{|c|c|c|c|}
\hline \multirow{2}{*}{ Molarity of NaCl (M) } & \multicolumn{4}{|c|}{ Molecular weight averages (x10 $\mathbf{4}^{\mathbf{4}}$ Da } \\
\cline { 2 - 4 } & $\mathbf{M w}$ & $\mathbf{M n}$ & $\mathbf{M p}$ \\
\hline 1 & 5.7 & 4.1 & 3.7 \\
\hline 2 & 6.6 & 4.8 & 4.9 \\
\hline 3 & 9.2 & 4.9 & 4.4 \\
\hline
\end{tabular}

\subsubsection{Melting point}

Since the melting point of crystallized compounds assesses also sample purity and is affected by molecular weight, melting points were measured for some fractions to proof relations with the previously measured molecular weight parameters [129]. All organic polymers like fucoidan melt and decompose when exposed to heat in an analysis called combustion analysis. Fucoidan fractions followed the same pattern with increasing the temperature; namely colour changing: yellow, brown and then to black and was reflected by a gas development. The temperature ranges between starting of melting till complete decomposition were 5-6 ${ }^{\circ} \mathrm{C}$ degrees for Fucoidan_1 and Fucoidan_M, while they were wide $\left(20^{\circ} \mathrm{C}\right)$ for Fucoidan_6. These data indicated and confirmed higher molecular weight of Fucoidan_6 and results obtained from molecular weight measurement. Table 7 summarizes a comparison among the phases at which the different fractions of fucoidan purified by 
immobilized TB changed their colour, melted and then decomposed with charring at higher temperatures.

Table 7: Comparison among the different fucoidan fractions purified by immobilized TB, regarding start, colour change and decomposition temperature points

\begin{tabular}{|c|c|c|c|}
\hline Fucoidan fraction & Start $\left({ }^{\circ} \mathbf{C}\right)$ & Color Change $\left({ }^{\circ} \mathbf{C}\right)$ & Decomposition $\left({ }^{\circ} \mathbf{C}\right)$ \\
\hline Fucoidan_1 & 130 & $132-133$ & 135 \\
\hline Fucoidan_6 & 140 & $153-156$ & 161 \\
\hline Fucoidan_M & 130 & $133-134$ & 136 \\
\hline
\end{tabular}

\subsubsection{Specific optical rotation}

Specific optical rotation $[\alpha]_{589}^{22}$ results, as shown also in Table 8, proved the levorotatory $(l)$ and asymmetric nature of fucoidan fractions. This confirmed the presence of $l$-fucose as a major stereoisomer of fucose monomer in fucoidan. Fucoidan fractions in addition to commercial analogue showed similar, $[\alpha]_{589}^{22}$ values in the range from $-117^{\circ}$ for Fucoidan_6 to $-130^{\circ}$ for Fucoidan_M. These values could be related also to fucoidan molecular weight and fucose content as an indirect relationship. Furthermore, these values are also consistent with the data reported previously in literature: $-123^{\circ}[43]$.

Table 8: Comparison among the different fractions of fucoidan regarding specific optical rotation All fucoidan fractions are $l$-form polymers.

\begin{tabular}{|c|c|}
\hline Fucoidan fraction & Specific optical rotation $[\alpha]_{589}^{22}$ \\
\hline Reference Fucoidan & -121 \\
\hline Fucoidan_1 & -128 \\
\hline Fucoidan_6 & -117 \\
\hline Fucoidan_M & -130 \\
\hline
\end{tabular}

\subsubsection{Monomeric composition}

Monomeric composition affects significantly fucoidan biological activities [43]. As Table 9 shows, fucose is the major monomer in all fucoidan fractions and represents more than $80 \%$ of the total monomers. Other monomers, such as galactose, uronic acid/glucose dimers were also detected. Yet, xylose was detected only in Fucoidan_1 and Fucoidan_6. Uronic acids/glucose dimers were only detected by MS; nevertheless, their quantities could not be accurately determined since there were 
no standards available in addition to their poor retention behaviors. These results confirmed that the monomeric composition of fucoidan was also dependent on the purification method and its molecular structure as well.

Table 9: Monomeric composition (\%) of different purified fucoidan fractions

\begin{tabular}{|c|c|c|c|}
\hline Composition & \% Galactose & \% Xylose & \% Fucose \\
\hline Fucoidan_1 & $7.4 \pm 0.06$ & $4.0 \pm 0.06$ & $88.59 \pm 0.03$ \\
\hline Fucoidan_6 & $8.99 \pm 0.25$ & $4.2 \pm 0.45$ & $86.8 \pm 0.45$ \\
\hline Fucoidan_M & $7.56 \pm 0.2$ & $-*$ & $92.43 \pm 0.2$ \\
\hline *: not detected
\end{tabular}

2.3.6. Spectroscopical identification of purified fucoidan fractions

\subsubsection{1. $\quad$ FT-IR}

Because of the complex and diverse structure of fucoidan, identification of fucoidan functional groups is always performed by IR [43,87,130,131]. Generally, it shows characteristic and typical IR bands for its functional structural building blocks, (e.g., $\mathrm{OH}$ group of its monomeric monosaccharides, $\mathrm{S}=\mathrm{O}$ and $\mathrm{C}-\mathrm{S}-\mathrm{O}$ of sulphate ester groups, $\mathrm{O}-\mathrm{C}-\mathrm{O}$ and $\mathrm{C}-\mathrm{O}-\mathrm{C}$ of glycosidic and intra-molecular linkages, at 3430,1220,830,1620,1020 $\mathrm{cm}^{-1}$, respectively).

FT-IR was used as an identification tool for fucoidan before and after application of the purification protocol. As revealed in Fig. 28, as an example, a comparison between IR bands for Fucoidan-PDD, crude fucoidan and the commercially-available pure fucoidan purchased from Sigma-Aldrich ${ }^{\circledR}$ (>95\% pure). Obviously, crude fucoidan showed an additional band at $1418 \mathrm{~cm}^{-1}$ which may belong to co-extracted impurities, e.g., alginate [132]. Being purified, fucoidan fractions had not any more this band as shown in Fucoidan_PDD. 


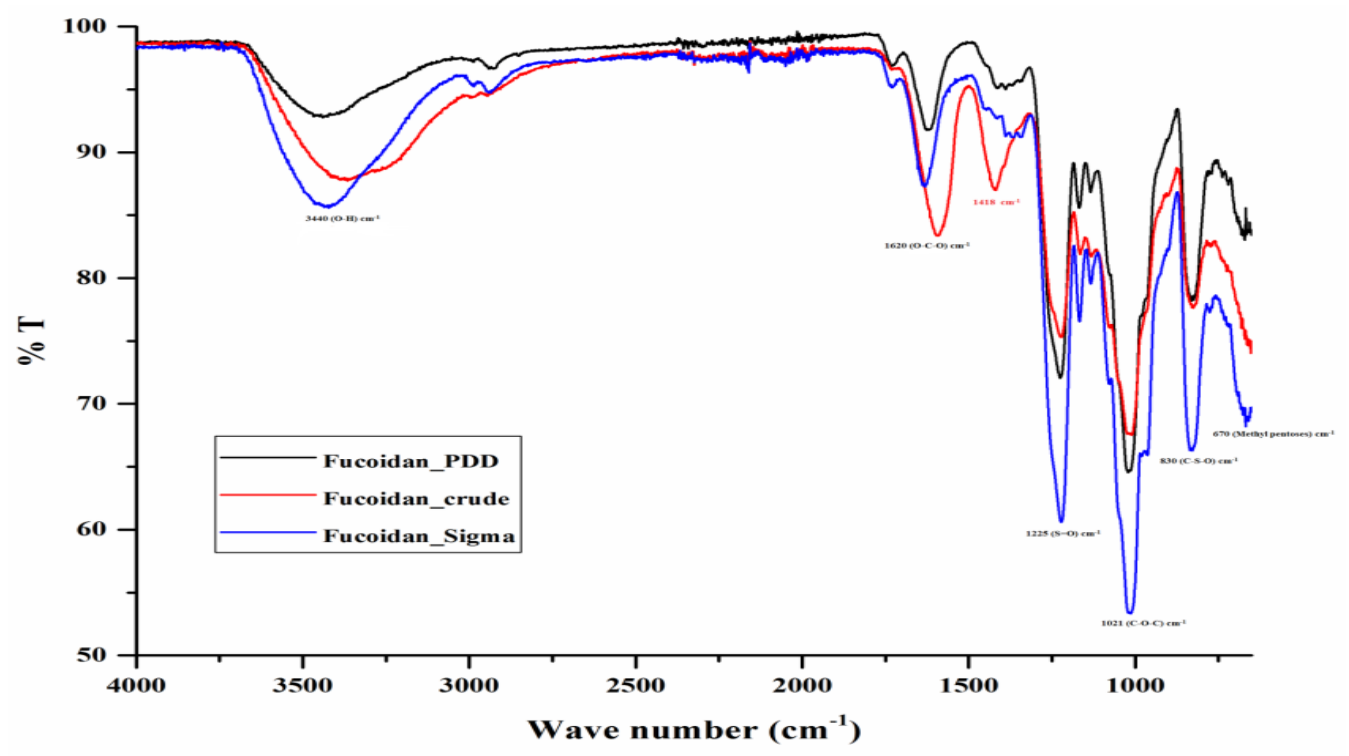

Fig. 28: FT-IR spectra of fucoidan before (Fucoidan_crude) and after (Fucoidan_PDD) in comparison with the commercially-available reference fucoidan purchased from Sigma-Aldrich ${ }^{\circledR}(>95 \%$ pure)

Fucoidan_PDD showed typical IR bands for OH, O-C-O, S=O, C-O-C, C-S-O groups as well as methyl pentoses of fucoidan. FT-IR of Fucoidan_PDD is only shown, as an example, for a simple overview.

\subsubsection{2. $\quad{ }^{1} \mathrm{H}-\mathrm{NMR}$}

${ }^{1} \mathrm{H}-\mathrm{NMR}$ of fucoidan fractions demonstrated poorly-translated spectra including commerciallyavailable purified fucoidan purchased from Sigma-Aldrich ${ }^{\circledR}$. Supposing that fucoidan is a polymer $\alpha$-(1-3)-linked L-fucopyranoside repeating unit, as previously hypothesized by Cumashi, et al. [14], as shown in Fig. 29, some structural features could be elucidated from NMR spectra. All fractions showed peaks of the shielded protons at around 1.2 and $2.15 \mathrm{ppm}$ as singlets (did not appear in commercial fucoidan), which could be assigned to $\mathrm{CH}_{3^{-}}$of L-fucose monomer and acetyl groups, respectively. Other peaks appeared slightly shifted between 3.8 and $4.5 \mathrm{ppm}$ assigned to $\mathrm{H}_{2}, \mathrm{H}_{3}, \mathrm{H}_{4}$ and $\mathrm{H}_{5}$. Anomeric proton $\mathrm{H}_{1}$ appeared highly down fielded at $5.2 \mathrm{rpm}$, confirmed $\alpha$-linked sugar monomers. 


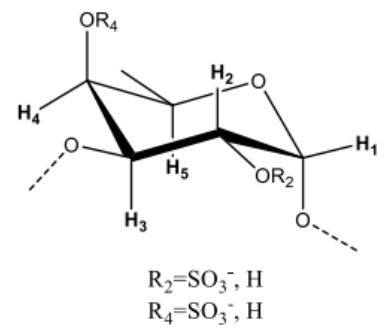

Fig. 29: Representation of an $\alpha$-(1-3)-linked L-fucopyranoside repeating unit of fucoidan, as previously described by Cumashi, et al. [14]

Fucoidan required further pre-treatment before NMR analysis for better and high quality spectra. This pre-treatment includes polymer hydrolysis to oligomers to simplify its structure which could be analyzed afterwards by different $1 \mathrm{D}$ and 2D-NMR techniques.

\subsubsection{Pharmacological activities}

\subsubsection{Anti-coagulant activity}

a. Activated partial thromboplastin time (aPTT)

Normally, aPTT records between 30-40 s, evaluates mainly the effect of anti-coagulants on the intrinsic pathway of blood coagulation system and to monitor patient's response to heparin therapy [133]. Results proved that fucoidan has a potential heparin-like or a heparinoid [134] anti-coagulant activity and interfered with intrinsic coagulation cascade. Coagulation times were significantly prolonged in comparison with the negative control $(0.9 \% \mathrm{NaCl})$ which recorded $41.8 \mathrm{~s}$.

Fig. 30 a shows that the purification process improved the anti-coagulant activity of crude fucoidan

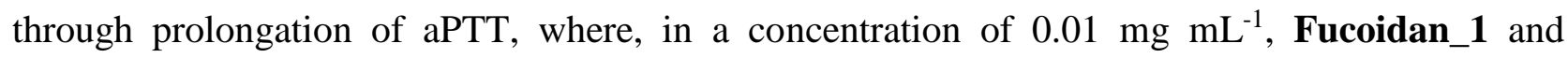
Fucoidan_6 prolonged coagulation times to 73 and 75 s, respectively, in comparison with 44.8 s for crude fucoidan. Fucoidan_M extended coagulation time, as well, but to a lesser extent to just only $51 \mathrm{~s}$ which was nearly similar to the reference analogue: $48.3 \mathrm{~s}$. The effect on aPTT did not help discriminate between the LMWF Fucoidan_1 and the HMWF Fucoidan_6. However, heparin of $0.005 \mathrm{mg} \mathrm{mL}^{-1}$ recorded $89.3 \mathrm{~s}$ indicating more than two times effectiveness than Fucoidan_6. In other experimental conditions, effect of Fucoidan_PDD was investigated on aPTT, as shown in Fig. 30 b. Fucoidan_PDD prolonged also aPTT significantly in a dose-dependent manner. 


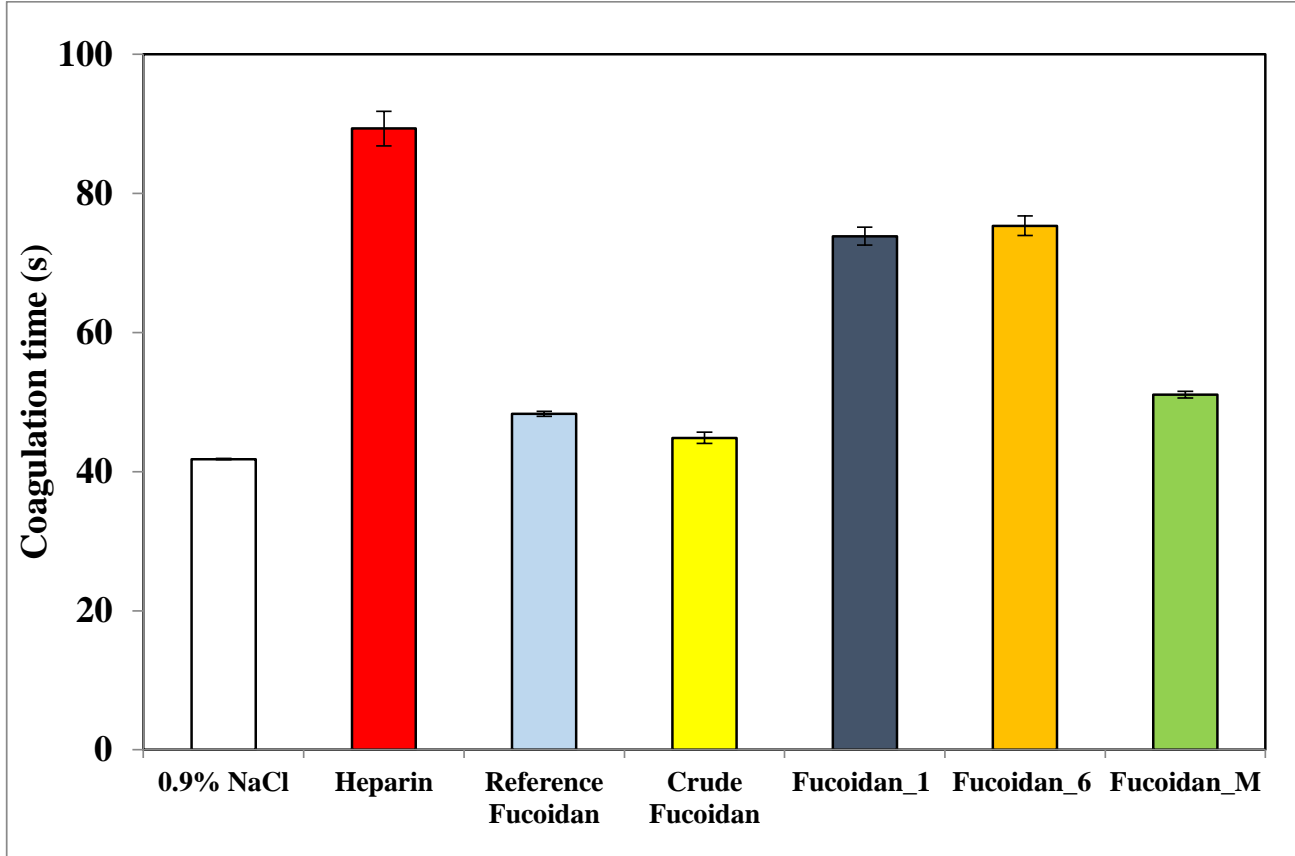

Fig. 30 a: Effect of different fucoidan fractions on aPPT at a concentration of $0.01 \mathbf{~ m g ~ m L} \mathbf{~}^{-1}$ $0.9 \% \mathrm{NaCl}$ and $0.005 \mathrm{mg} \mathrm{mL}^{-1}$ heparin were chosen as negative and positive controls, respectively $(n=3)$.

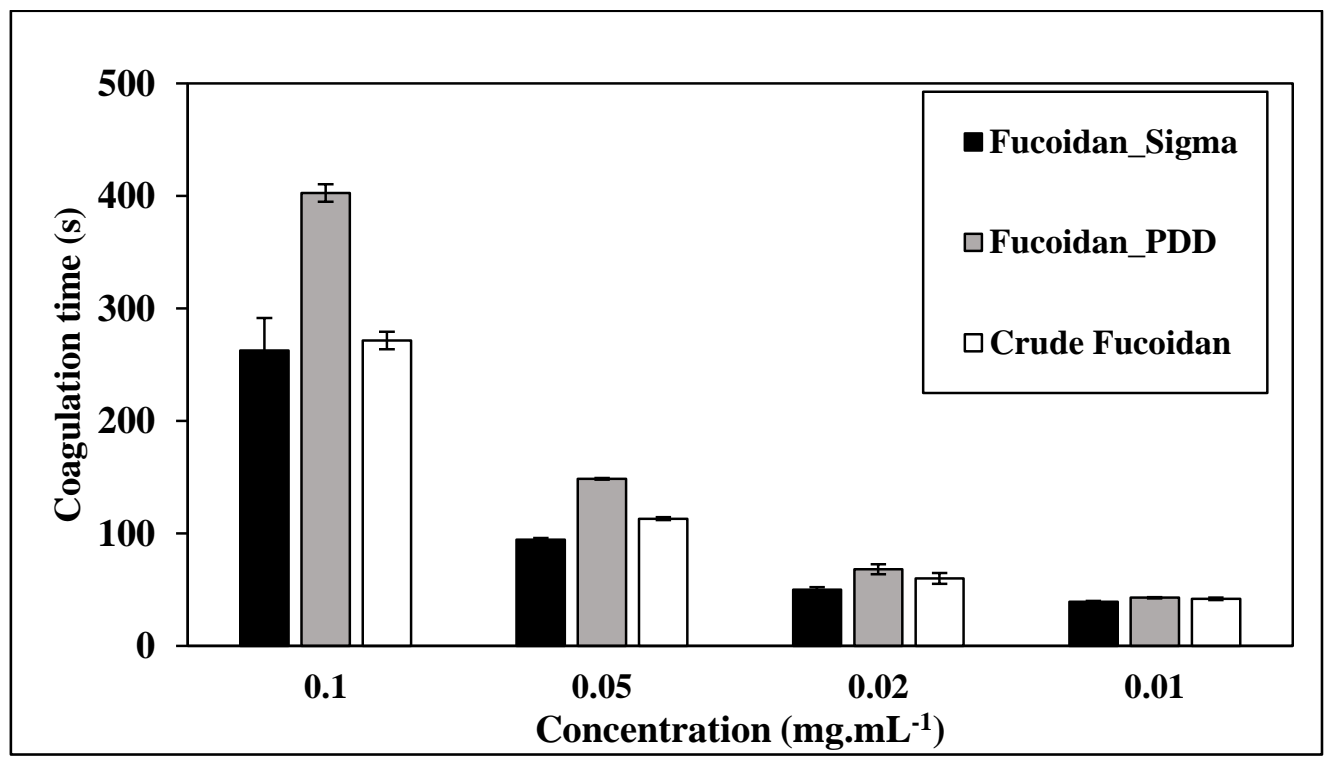

Fig. 30 b: A dose-dependent effect of Fucoidan_PDD on aPTT

Effect was compared with crude fucoidan and reference fucoidan (Fucoidan_Sigma) $(n=3)$. 
b. Prothrombin Time (PT)

PT is usually used to evaluate the effect of anti-coagulants (e.g., warfarin) on the blood extrinsic coagulation pathway. It is obvious in Fig. 31 that all types of fucoidan were not able to prolong PT significantly in comparison with the negative control. These data were consistent with the mechanism of fucoidan as an anti-coagulant, which has a heparin-like effect and mediated by serine protease thrombin enzyme and/or heparin cofactor II inhibition [135] present in common and intrinsic pathways.

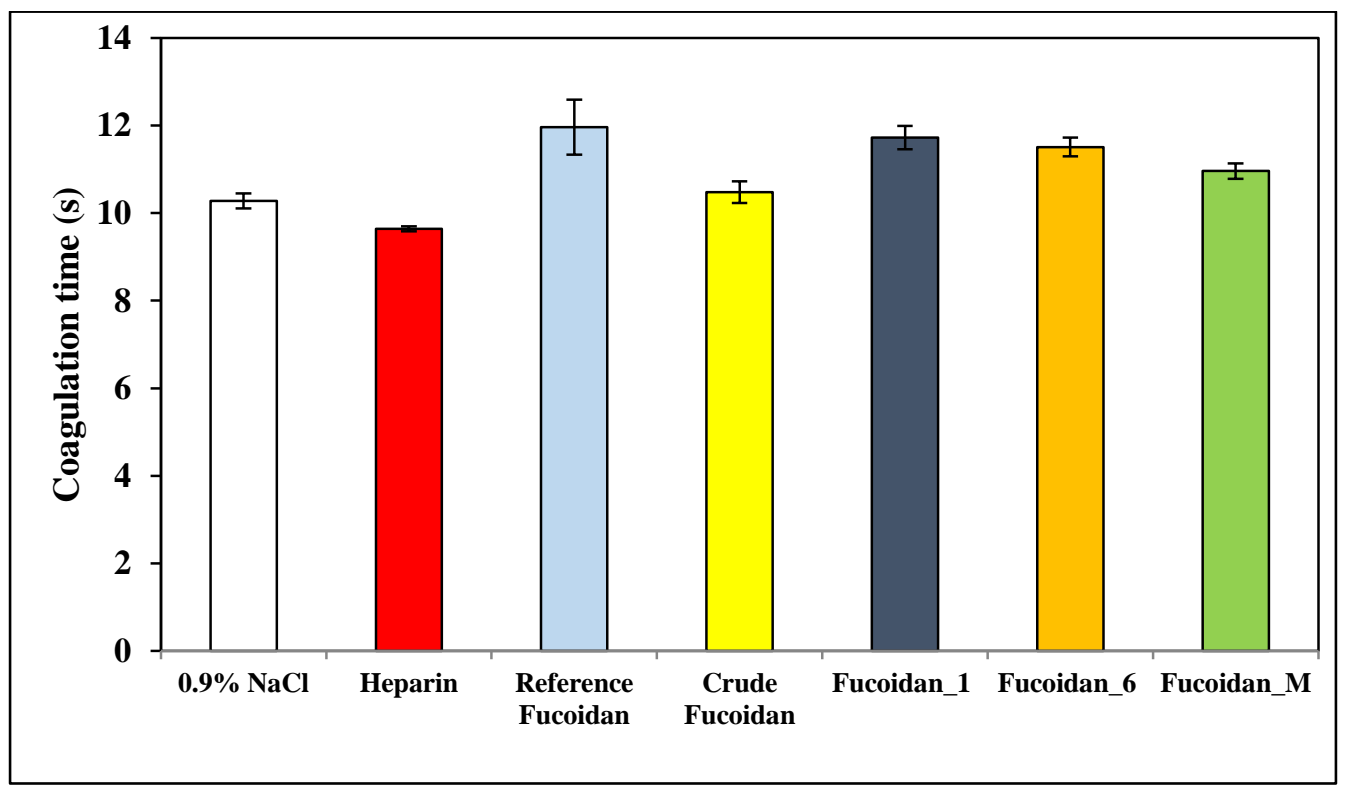

Fig. 31: Effect of different types of fucoidan on PT at a concentration of $0.01 \mathbf{~ m g ~ m L} \mathbf{~}^{-1}$

$0.9 \% \mathrm{NaCl}$ and $0.005 \mathrm{mg} \mathrm{mL}^{-1}$ heparin were used as negative and positive control, respectively $(\mathrm{n}=3)$.

c. Thrombin Time (TT)

TT studies specifically thrombin function and the effect of anticoagulants against fibrinogen transformation into non-soluble fibrin threads. This step is enzymatically catalyzed by thrombin (Factor IIa). The reference range for the thrombin time is usually less than $20 \mathrm{~s}$ [136]. Results, as shown in Fig. 32, revealed a similar pattern as in Fig. 30 a, where Fucoidan_6 also demonstrated the highest activity and recorded the longest time required for blood coagulation. Fucoidan_1 and Fucoidan_6 prolonged coagulation time significantly to 47 and 66 s, respectively, compared to 19.27 s for the negative control, while Fucoidan_M showed the weakest effect on coagulation time. 
It increased the coagulation time only to $23.5 \mathrm{~s}$ compared with 31.3 and $29.2 \mathrm{~s}$ for reference and crude fucoidan, respectively.

The anti-thrombin differences revealed the importance of sugar composition, molecular weights, and structural comfortability, where higher galactose, an enough long sugar chain and comfortable structure found in Fucoidan_6 were more critical than sulphate content for thrombin inhibition $[43,137]$. These conclusions could be confirmed by the weak effect of the low molecular weight of Fucoidan_1and Fucoidan_M, despite of their higher sulphate contents. More sulphate content than the required threshold could also lead to a decrease in the anti-thrombin activity $[138,139,140]$. Moreover, in a dose dependent manner, the effect of Fucoidan_PDD as an anti-thrombin was investigated separately, as shown in Fig. 32 b. Results confirmed previous conclusions regarding the importance of polymer molecular weight and chain comfortability for anti-thrombin activity.

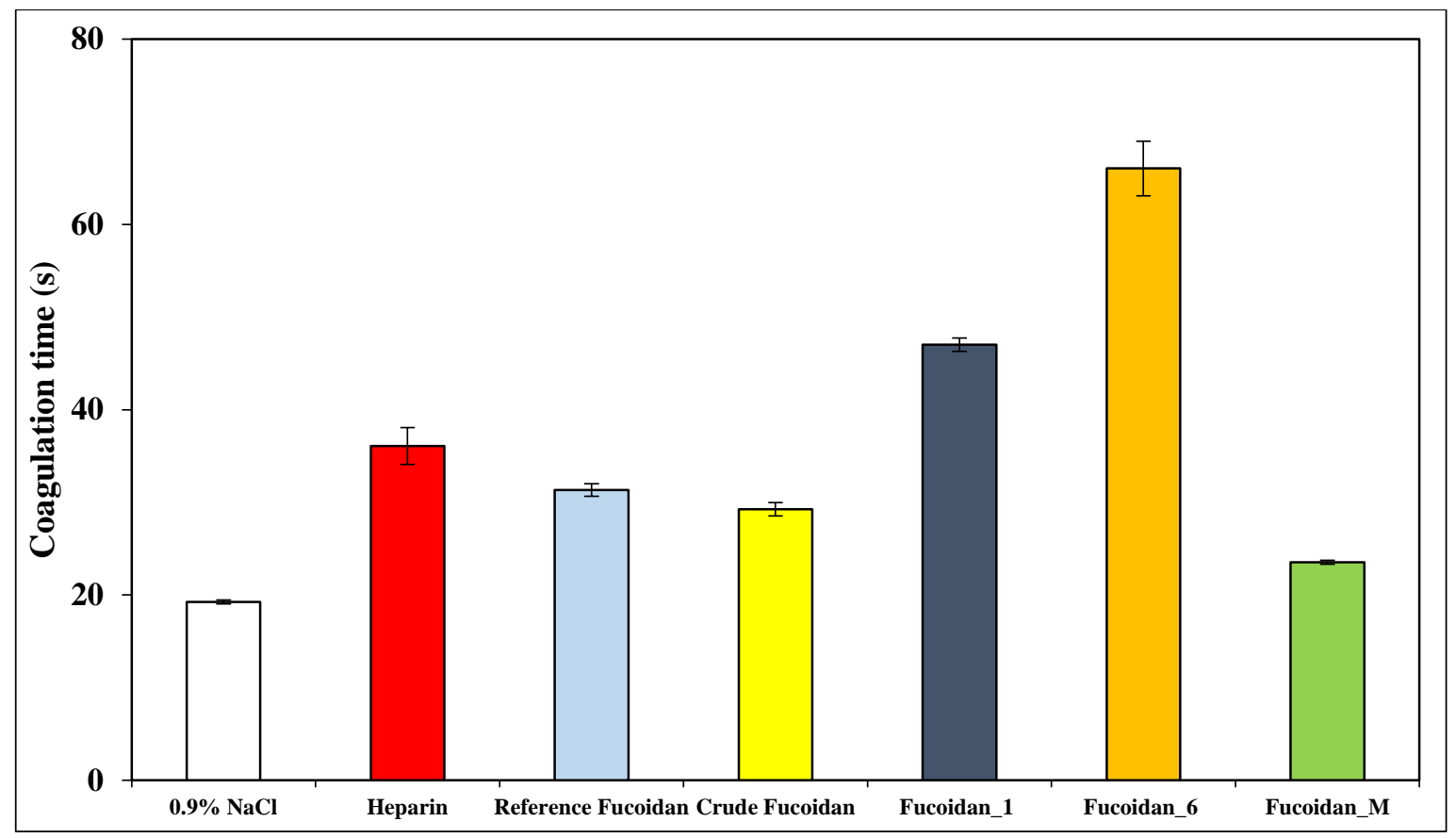

Fig. 32 a: Effect of different fucoidan fractions on TT at a concentration of $0.01 \mathbf{~ m g ~ m L}^{-1}$

$0.9 \% \mathrm{NaCl}$ and $0.005 \mathrm{mg} \mathrm{mL}^{-1}$ heparin were tested as negative and positive control, respectively $(\mathrm{n}=3)$ 


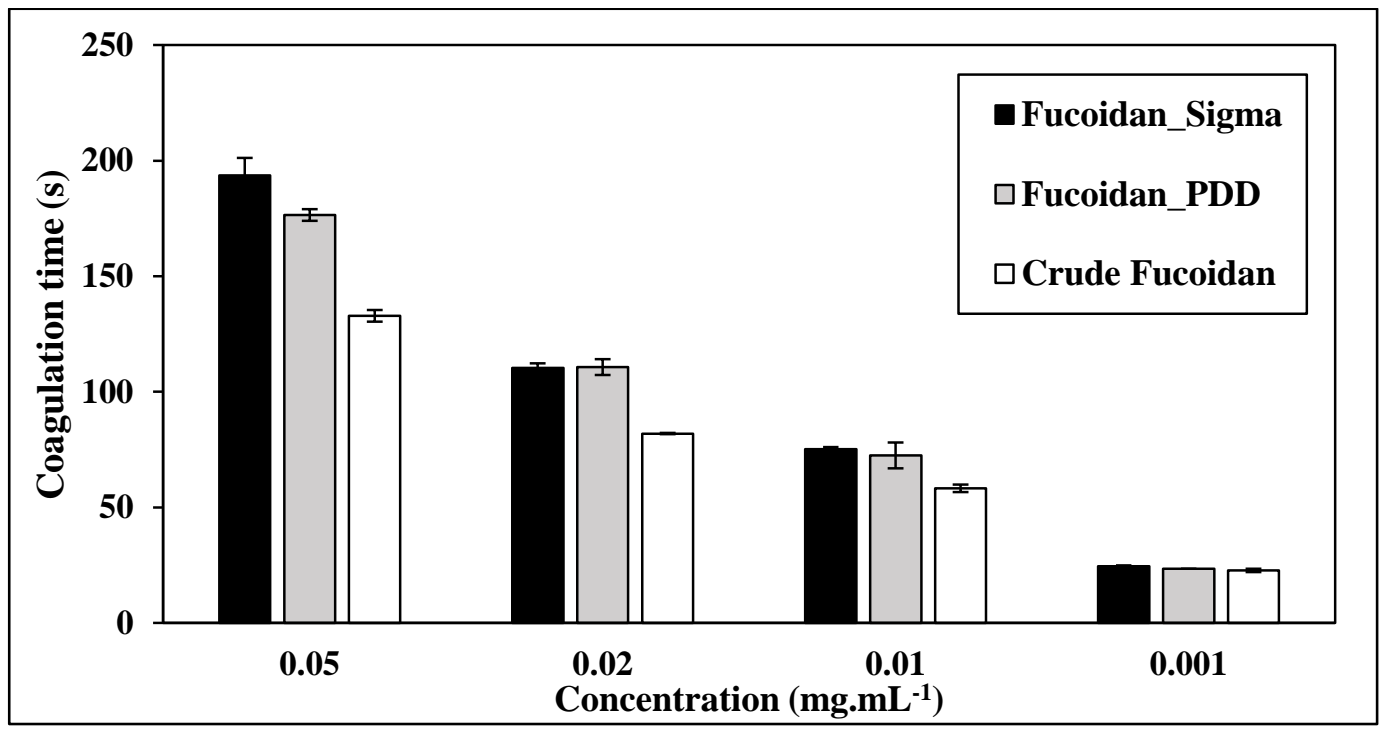

Fig. 32 b: A dose-dependent anti-thrombin effect of Fucoidan_PDD

The effect on TT was studied in a comparison with crude and commercially-pure fucoidan (Fucoidan_Sigma) $(\mathrm{n}=3)$.

\subsubsection{Anti-viral activity}

Herpes simplex virus type 1 or HSV-1 is a human dsDNA virus that infects human and transmitted through oral-to-oral contact and causes cold sores. According to WHO, 67\% of world population whose age under 50, have HSV-1 [141]. Fucoidan exhibits its anti-viral activity against HSV-1 mainly through interference with viral replication [134], adsorption and penetration through cellular surface modification, and subsequently, it prevents the viral syncytium formation [63]. For this action, sulphate ester groups' position and content are critical for the anti-HSV-1 activity [134]. Consistent with literature, the fraction with the highest sulphate content, Fucoidan_M, exerted a highly potent anti-viral effect in comparison with other types. Fig. 33 demonstrates the anti-viral activity (\%) with different serial dilutions for the different fucoidan fractions against HSV-1 and followed by calculated $\mathrm{IC}_{50}$ values in Table 10. IC $_{50}$ varied among different fucoidan fractions from

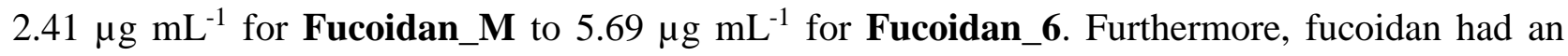
advantageous less cytotoxic effect against Vero B cell culture than the commercially-used reference anti-viral aciclovir. 


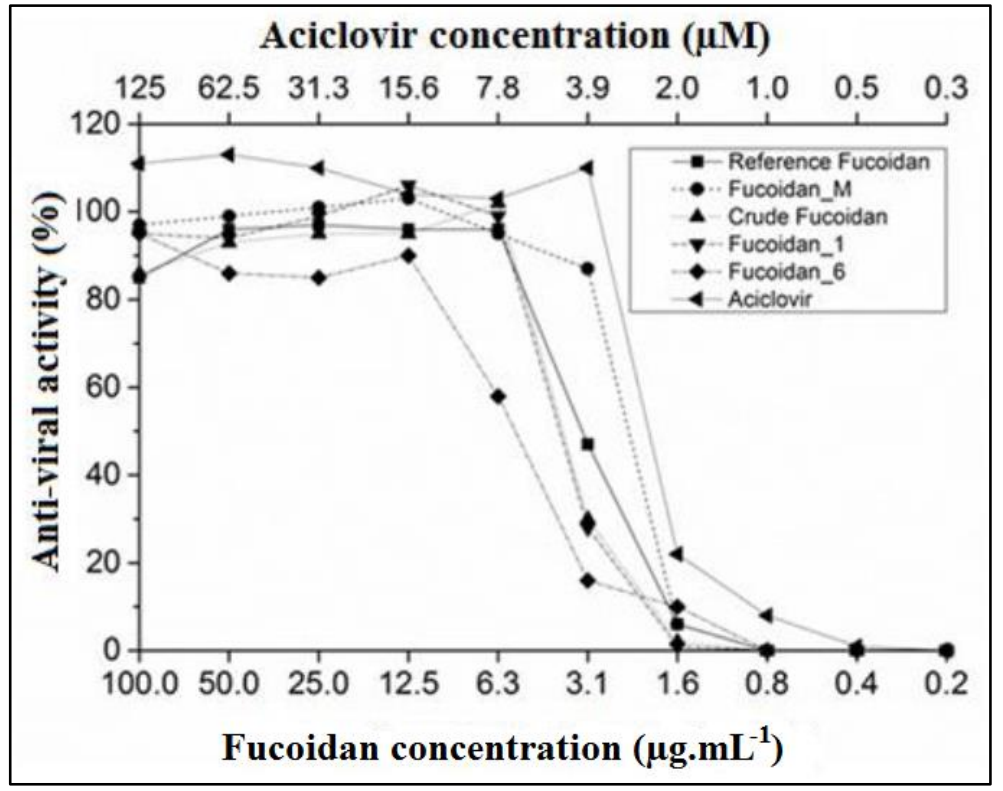

Fig. 33: Comparison between the anti-viral activities of fucoidan fractions against HSV-1

The activity was compared with the commercial analogue purchased from Sigma-Aldrich ${ }^{\circledR}$ (Reference fucoidan) against HSV-1. Aciclovir was used as a positive control.

Table 10: $\mathrm{IC}_{50}\left(\mu \mathrm{g} \mathrm{mL}^{-1}\right)$ of different fucoidan fractions isolated and purified from $F$. vesiculosus against HSV-1 in comparison with aciclovir

\begin{tabular}{|c|c|}
\hline & IC $_{\mathbf{5 0}}\left(\boldsymbol{\mu} \mathbf{g} \mathbf{~ m L}^{-\mathbf{1}}\right)$ \\
\hline Reference Fucoidan & 3.3 \\
\hline Fucoidan_1 & 4.09 \\
\hline Fucoidan_6 & 5.69 \\
\hline Fucoidan_M & 2.41 \\
\hline Crude fucoidan & 3.99 \\
\hline Acicolvir & 0.52 \\
\hline
\end{tabular}

\subsubsection{Anti-oxidant activity (DPPH Radical scavenging activity)}

Radicle scavenging anti-oxidant activity of fucoidan was investigated its ability to capture the free radical DPPH. As shown in Fig. 34, all fractions of fucoidan, including crude and reference fucoidan, were DPPH inactive scavengers. The results are inconsistent with literature that confirmed the DPPH radicle antioxidant activity of fucoidan of $F$. vesiculosus $[87,88]$. Most of the published articles discussed the potential anti-oxidant activity of fucoidan applied a pre-treatement step with acetone, water/chloroform/methanol mixture and did not use formaldehyde, because of its toxicity. Formaldehyde functions to polymerize polyphenols which have a strong relationship with the 
potential anti-oxidant activity of fucoidan. Diaz-Rubio et al. have determined significant polyphenol content (960 mg phloroglucinol $100 \mathrm{~g}^{-1}$ dry weight) in commercial fucoidan from $F$. vesiculosus (Sigma-Aldrich, Spain) [89]. Since formaldehyde was applied in pre-treatment steps, anti-oxidant inactivity results for all fucoidan fractions are reasonable.

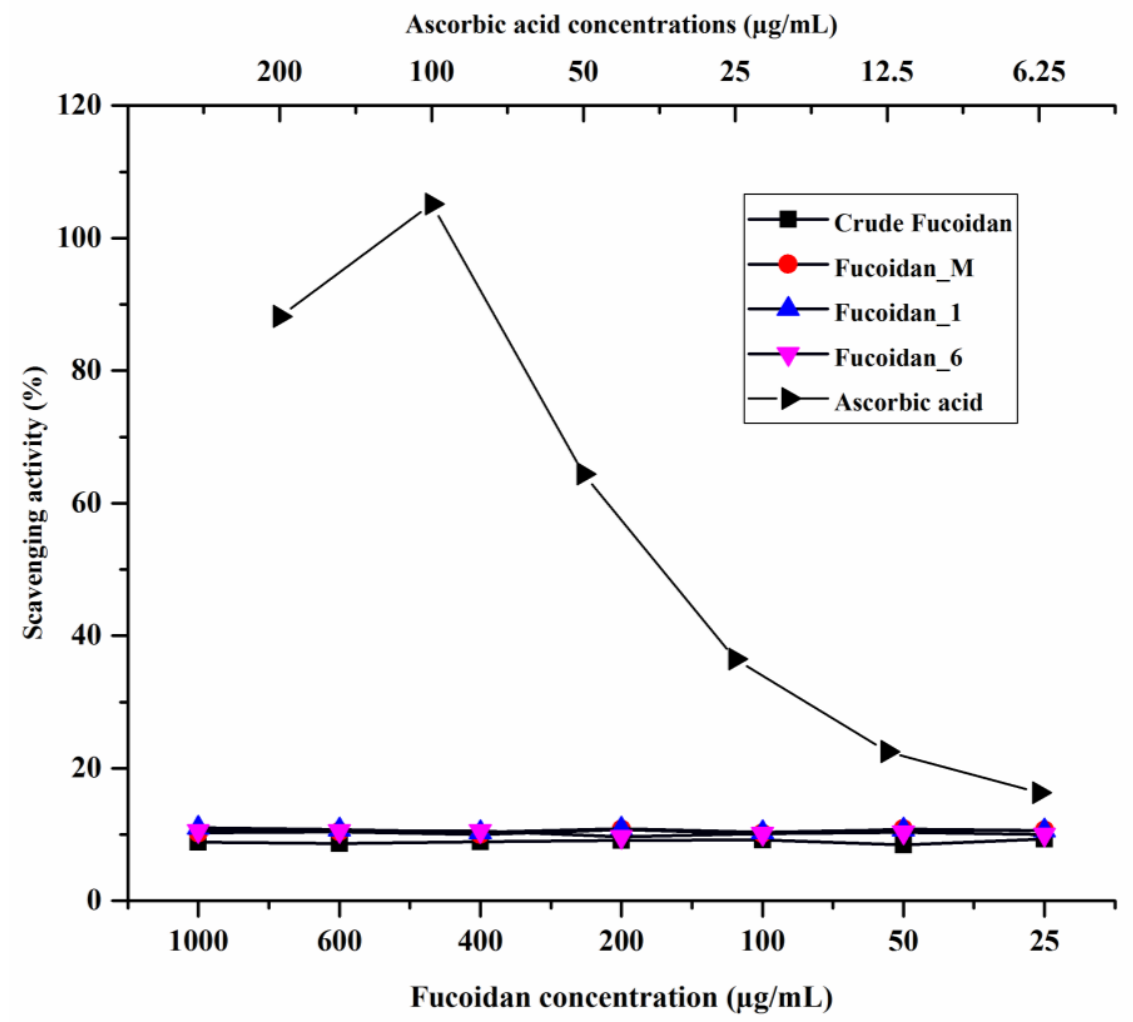

Fig. 34: Antioxidant activity of different fucoidan fractions in comparison with ascorbic acid The figure demonstrates the potential DPPH radicle scavenging activity of ascorbic acid. However there is no activity for all fucoidan fractions.

\subsubsection{Anti-microbial activities}

a. Anti-fungal activity

Fucoidan's anti-fungal activity was investigated against a number of candida species, such as $C$. albicans, C. glabrata and C. tropicalis. All fucoidan fractions did not show any activity and failed to eradicate the fungal growth. This means that fucoidan could not affect sites of actions of common anti-fungal agents, such as sterols synthesis in cell membrane like polyene anti-fungal drugs or inhibition of cytochrome $\mathrm{P}_{450}$-dependent enzymes as in azole anti-fungal drugs. 
b. Anti-bacterial activity

As in anti-oxidant and anti-fungal investigations, fucoidan was non-toxic to either Gram +ve as $S$. aureus or Gram -ve bacteria such as E. coli.

2.3.8. Process scaling-up and application of optimized conditions

Scheme 2 demonstrates the downstream fucoidan extraction and purification processes. The process initiated with algae harvesting from its natural habitat, then pre-treatment, extraction of crude fucoidan and finally purification with immobilized TB and PDD at pH 6. In addition, obtained yields from the different fucoidan fractions are mentioned in Fig. 35.

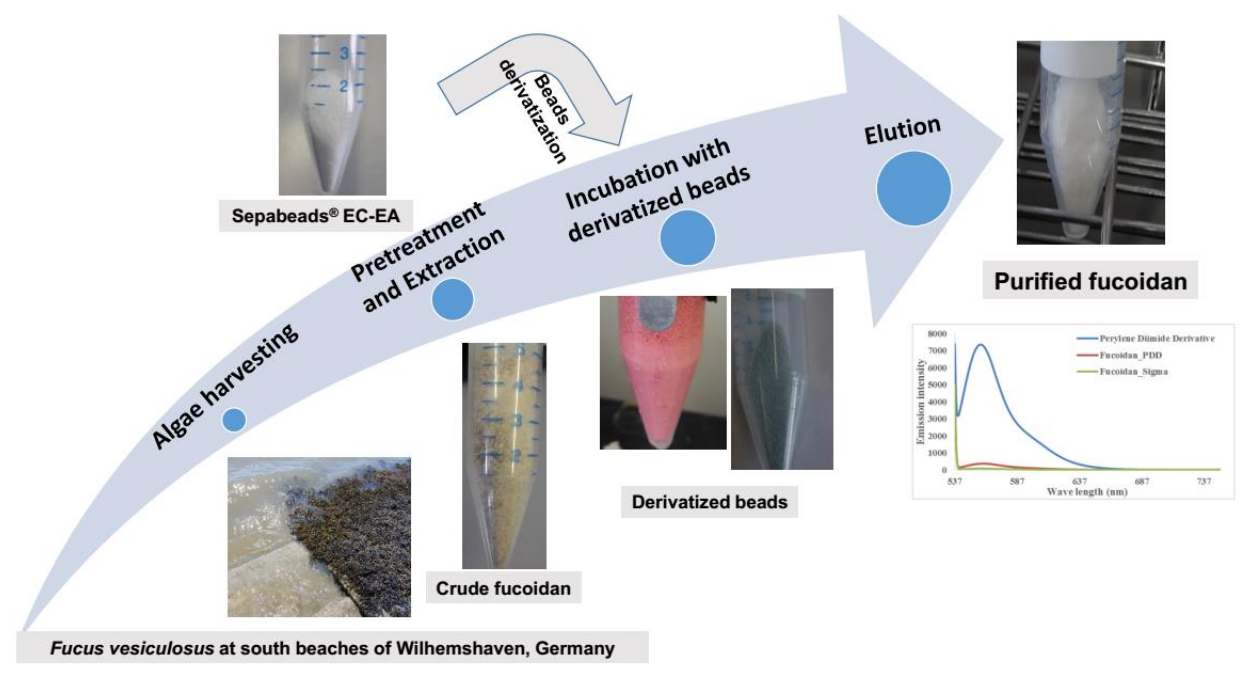

Scheme 2: Graphical summary for fucoidan purification process from $F$. vesiculosus

The downstream process includes algae harvesting, pre-treatment, extraction and purification with either TB- or PDD-derivatized beads. 


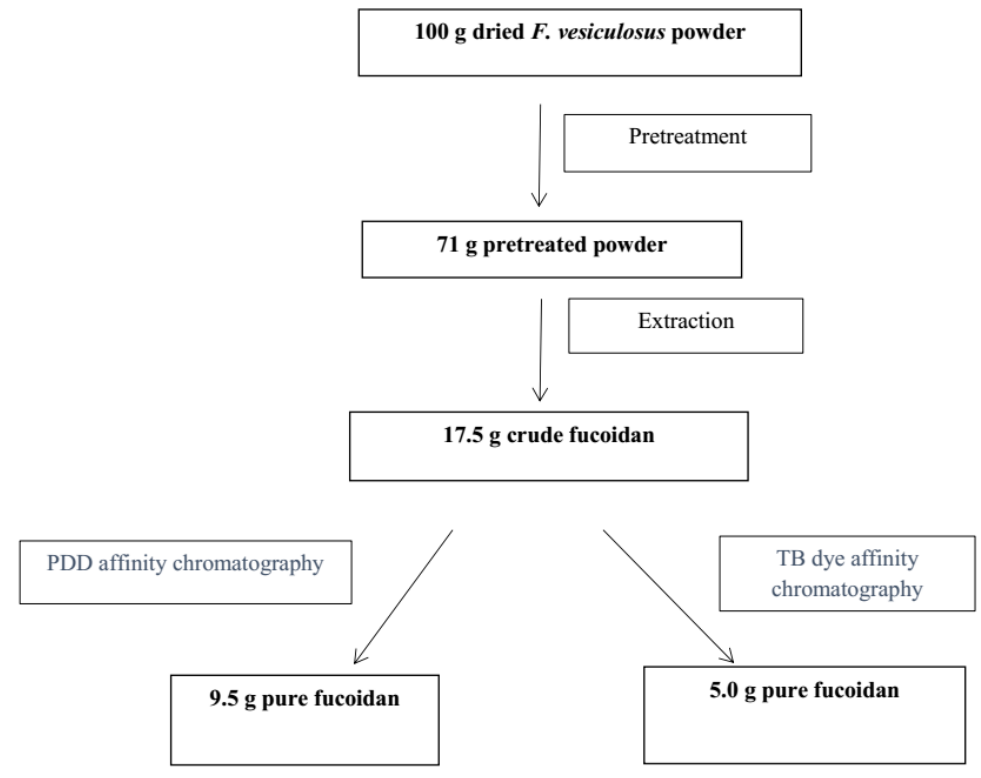

Fig. 35: Downstream process for fucoidan extraction and purification by either TB- or PDD-derivatized beads at pH 6

The processes resulted in production of $5.0 \mathrm{~g}$ and $9.5 \mathrm{~g}$ of purified fucoidan after purication with TBand PDD-derivatized beads, respectively.

Moreover, fucoidan content (\%) or purity was investigated using TB and Heparin Red ${ }^{\circledR}$ Ultra assay methods. Fucoidan purity was improved in comparsion with crude fucoidan by 1.69 and 1.4 fold after purification through TB- and PDD-immobilized beads, respectively. Fig. 36 shows that after purification by PDD-immobilized beads, the investigated concentrations of Fucoidan_PDD were similar to the commercial product regarding fucoidan content. Concentration of Fucoidan _PDD relative to standard was derived from the calibration curve. Purified fucoidan (Fucoidan_PDD) showed 97\% purity relative to the reference fucoidan (Fucoidan_Sigma). Furthermore, alginate, the major contaminant in fucoidan crude extract, did not show with Heparin Red $^{\circledR}$ any fluorescence quenching behavior (Fig. 77). Since the response of Heparin Red is sensitive to the charge density of the polysulphated polysaccharide and the sulphation degree of Fucidan_PDD is lower than that of the reference fucoidan (Table 5), the actual purity of Fucoidan_PDD could be even higher. 


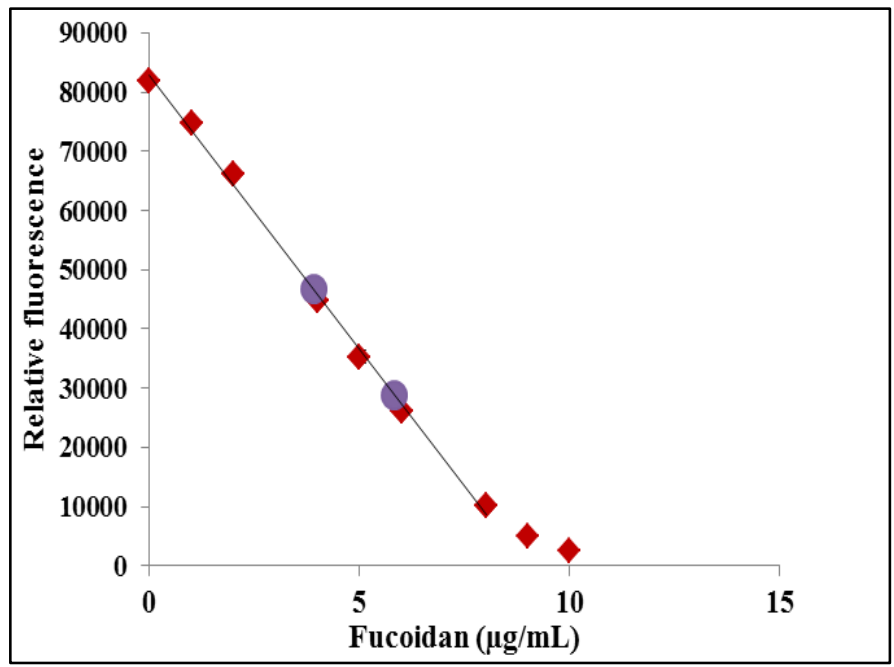

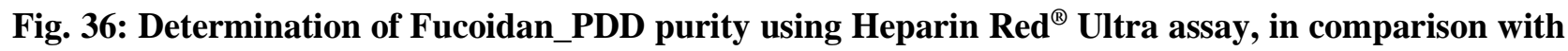
the commercial standard product $\left(>95 \%\right.$ pure) purchased from Sigma-Aldrich ${ }^{\circledR}$

Fucoidan content recorded $97 \%$ relative to the standard product. However, it was $69 \%$ in case of crude fucoidan (Fucoidan_A). Determination was compared with a calibration curve of the reference fucidan (red diamonds), using linear regeression for the range 0.0-8.0 $\mu \mathrm{g} \mathrm{mL}^{-1}\left(\mathrm{R}^{2}=0.9974\right)$. Fucoidan_PDD samples $\left(0.4\right.$ and $\left.0.6 \mu \mathrm{g} . \mathrm{mL}^{-1}\right)$ were prepared and the fluorescence determined (violet circles). 


\subsection{Conclusion and Prospectives}

Pre-treatment with different solvents succeeded to reduce the co-extraction of other extraneous algal constituents (e.g., lipids, pigments, mannitol, and polypehenols). In addition, extraction in acidic condition at higher temperature with reflux improved the production yield of extracted crude fucoidan. Interestingly, application of the recently-developed dye affinity chromatography protocol was possible to purify fucoidan from its crude extract. Different conditions and factors were studied to optimize the process. Moreover, TB-derivatized beads had the selectivity to capture fucoidan even without fucoidan precipitation with ethanol. This procedure resulted in production of a new fucoidan fraction; Fucoidan_M. However, the results revealed that the process suffered from some disadvantages, such as long incubation periods and acidic $\mathrm{pH}$ were required to achieve the maximum loading capacity of the immobilized dyes, in addition to the inherent problems related to both thiazine dyes represented by limited number of adsorption sites and fucoidan as slower and different diffusion rates of fucoidan polymers and beads porosity, as well.

These challenges were overcome by synthesis and immobilization of a Heparin Red $^{\circledR}$-structurally related PDD which has more available adsorption sites than thiazine dyes. Immobilized PDD succeeded to capture similar quantities of fucoidan in a shorter incubation period at a slightly acidic condition. The second challenge with fucoidan diffusion rates was resolved by performing of the adsorption phase under an external pressure to push the fucoidan molecules much faster to the adsorption sites by developing a FPLC protocol. The last technique helped also to automate and scale-up of the purification process.

Future work should focus on using non-porous beads with more surface reactive sites. In addition, optimization of FPLC protocol in adsorption phase and in elution phase should be performed. It was clear that the maximum loading capacity of the column was not reached and this means that there were still available adsorption sites. The concentration of injected sample could be increased using a $5 \mathrm{~mL}$ injection loop to load the column with much fucoidan content considering the breakthrough curve of the purification process. Moreover, elution and fucoidan recovery (\%) could be optimized by varying the eluent flow rates, applying warmer temperatures and another force instead of the vigorous shaking used in the batch process. Furthermore, application of such sensitive technique could be performed in fucoidan capture and analysis in different raw synthetic dosage forms in the presence of other drug excipients. These experiments could achieve great success in pharmacokinetic studies toward a FDA approval of fucoidan as a medicament. 



\section{Development of Axenic Protoplast and Callus-like Cultures from $F$. vesiculosus}

\subsection{Introduction}

\subsubsection{Marine biotechnology challenges}

Seaweed farming through aquaculture (e.g., tanks, cages) was established to provide the expanding global market with its demands. However, major problems, such as control of epiphytes, fouling algae, grazing animals, and low levels of planting stock are greatly limiting industrial-scale seaweed cultivation. Moreover, seaweed farms occupy large areas hampering shipping and fishing and creating a lot of social problems as a result. Furthermore, seaweeds harvesting affects natural marine and terrestrial ecosystems negatively [143]. Expansion of market requirements from seaweeds and their valuable products needs new technologies, especially these eco-friendly techniques (e.g., marine biotechnology), as alternative tools.

Marine biotechnology has drawn a special interest in the last few decades following advances developed in plant and animal fields $[1,145]$ as a potential tool for the discovery and development, of marine-derived compounds with biomedical applications. It involves tissue culture, protoplast isolation, cell fusion and gene transfer [146]. Its advantages include the production of a high-yield with improved quality products from fast growing and diseases-free strains [147]. However, the challenge facing the marine biotechnology industry in the next millennium is to:

- identify new sources of marine bioproducts;

- develop novel screening technologies;

- provide a sustainable source of supply; and

- optimize production and recovery of the bioproducts [148].

Microalgae have rapid growth rates, simple structures and ability to adapt in different cultivation conditions [149]. These factors led to interesting advances in cultivation techniques including isolation and purification of microalgae from natural habitat and well-characterized nutritional requirements. As a result, a possible establishment of a lot of microalgae single strain cultures either in a closed or open system. Therefore, microalgae have been seen as potential candidates in biotechnological researches and industrial applications for production of biofuels and valuable bioactive compounds. These achievements encouraged the performance of similar techniques for the more developed and complicated seaweeds in the last few decades. 
3.1.2. Marine microbes and macroalgae tissue culture

Adding to the complex structure of marine macroalgae, marine microbes live in a symbiotic relationship with seaweeds. They produce vital compounds for macroalgae immune system resulting in improving the host resistance $[150,151]$ or function in the food chain cycle, as well as nutrients assimilation. Macroalgae host various marine microbes and diatoms providing them protection from tides and predators. Moreover, marine bacteria are very important for macroalgae development and morphogenesis, such as the green algae Ulva mutabilis through a cell to cell communication signals $[152,153]$.

A crucial requirement for bioprocess technologies for production of valuable marine products is the development of an axenic, fast-growing culture before scale-up to various bioreactor cultivations $[145,154]$. The surface of seaweeds is heavily infested by various microbial and larger epiphytes. Some of these organisms are implanted in cell walls and between meristematic cells which are frequently located on the surface and will be impaired upon chemical treatment [132]. Meristematic cells are highly divided cells and seen as a potential tissue for starting various marine biotechnology techniques. Due to microbes' diversity, an efficient and easily-applicable surface sterilization protocol is needed. This protocol should combine more than one strategy to combat against the different marine populations. In contrast to terrestrial plant, thallus tissue is mainly composed of delicate parenchyma tissues. Application of sodium hypochlorite $(\mathrm{NaOCl})$ and $70 \%(\mathrm{v} / \mathrm{v})$ ethanol are commonly-used as disinfectants in terreistrial plant surface sterilization protocols eradicating most of the symbiotically-lived microorganisms. However, they could bleach and destroy macroalgae parenchymal tissues and thereby reducing photosynthetic ability and viability.

Several strategies could be applied to obtain marine aseptic cultures, such as using ultraviolet radiation [155], povidone iodine and broad spectra antibiotics [154]. Nevertheless, these strategies are ineffective, time-consuming; especially, when used as separate procedures and their doses should be further controlled. Moreover, the effect of surface sterilization procedures on tissue vitality should be evaluated afterwards before starting cultivation as well. Trials to develop marine STC are still in their infancy compared to PTC for production of valuable compounds [156]. This status could be related to tissue structure complexity found in macroalgae in addition to nutritional and long-term aseptic requirements. These factors determine the part within the thalli from which the explant is excised and the degree of relationship between marine microbiomes and macroalgae. 
3.1.3. Development of marine macroalgal cultures

Callus-like cultures are well-known cell lines for decades as friable totipotent non-differentiated cells. These lines used as production systems for a lot of bioactive compounds in PTC (e.g., paclitaxel and Scopolamine) [157]. However, marine callus-like growth appears usually as filaments originating from exposed cut meristematic active cells, as shown in Fig. 37. Through further culture optimization (i.e., medium solidity, photoperiod regime and phytohormones), organogenesis and development into a plantlet were possible [158].

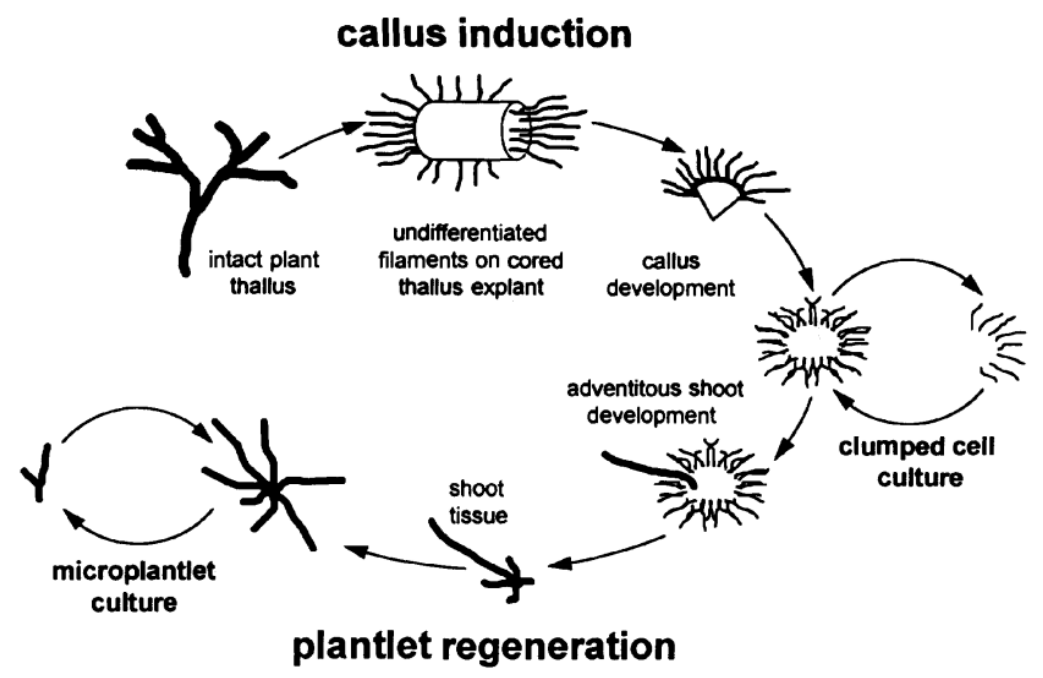

Fig. 37: Callus and plantlet regeneration from an intact marine macroalgae thallus of Agardhiella subulata [158]

Moreover, Huang and Rorrer established a microplantlet suspension culture from the red algae Agardhiella subulata in a stirred tank photobioreactor [159]. Such achievements could lead to elicitation of a diverse array of valuable natural compounds from a reliable supply of a macroalgae cell biomass.

Another relatively new biotechnological strategy to develop a marine biomass culture is protoplast cultivation. Although, protoplast isolation in terrestrial plants was known since 1960s, it has only been recently shown in seaweeds. Marine protoplasts have cell membranes but lack their polysaccharide cell walls. Macroalgae cell walls could be removed through a specifically enzymatic attack to both the microfibrillar and polysaccharide matrix components (i.e., alginate, galactans and fucoidan) [160,161]. Isolation of protoplasts from red and brown algae are more difficult than in green algae, because of their cell wall resistance to cellulase and pectinase [162]. Reddy and Fujita 
isolated protoplast from three species of green algae and succeeded to regenerate plantlet from axenic protoplast culture of Enteromorpha linza [163].

\subsubsection{Growth requirements and previously-performed trials}

Axenic as well as vital explants could be cultivated and induced to give a callus-like growth and continue further to develop a cultivation in a closed system bioreactor. Different growth conditions and factors could be applied and affect culture growth, as demonstrated in Fig. 38.

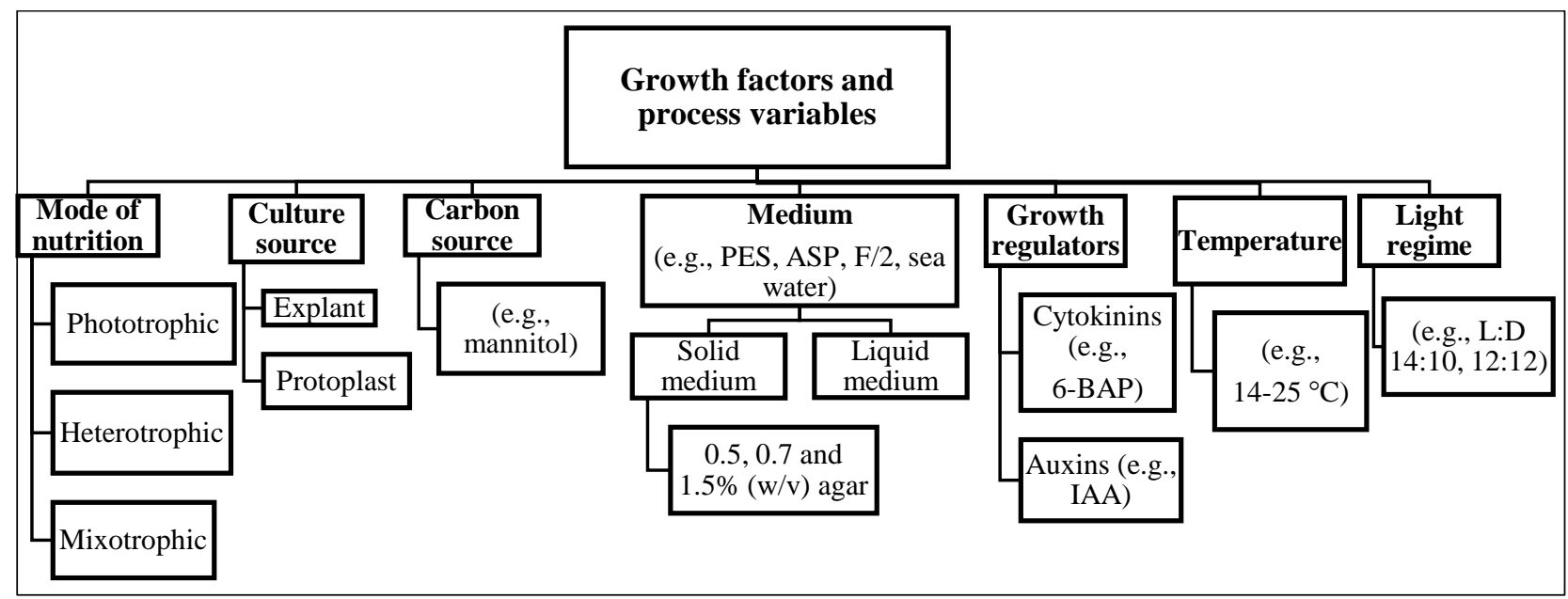

Fig. 38: Summary of previously-applied growth conditions and variables to develop marine macroalgal cultures

In more details, several experiments were previously mentioned for development of a callus-like growth and plantlet regeneration from seaweeds, as shown in Table 11. Nevertheless, few of them could succeed to produce valuable products in bioreactor systems (e.g., Laminaria saccharina suspension culture system) which biosynthesized three bioactive hydroxy fatty acids deriving from $\omega-6$ lipoxygenase oxidation [158]. 
Table 11: Some selected trials with callus cultures and plant regeneration in marine macroalgae organisms

\begin{tabular}{|c|c|c|c|c|}
\hline $\begin{array}{c}\text { Macroalagae } \\
\text { class }\end{array}$ & Parameters & Organism & Medium & Ref. \\
\hline \multirow[t]{6}{*}{$\begin{array}{l}\text { 1. Brown } \\
\text { algae }\end{array}$} & $\begin{array}{l}\text { - } \mathrm{T}: 12{ }^{\circ} \mathrm{C} \\
\text { - } \mathrm{L}: \mathrm{D}(8: 14)\end{array}$ & $\begin{array}{l}\text { Apical } \\
\text { meristematic } \\
\text { cells of } F \text {. } \\
\text { vesiculosus }\end{array}$ & Sterile sea water & [164] \\
\hline & $\begin{array}{l}\text { - } \mathrm{T}: 14^{\circ} \mathrm{C}, \\
\text { - } \mathrm{L}: \mathrm{D}(14: 10), 2000 \mathrm{Lux} \\
\text { - } 3 \% \text { Mannitol }\end{array}$ & $\begin{array}{l}\text { Dictyosiphon } \\
\text { foeniculaceus }\end{array}$ & $\begin{array}{l}\text { ASP } 12-\mathrm{NTA}, 0.1 \% \text { yeast, } \\
1.5 \% \text { agar (ASP-C-1 } \\
\text { medium) }\end{array}$ & [165] \\
\hline & $\begin{array}{l}\text { - } \mathrm{T}: 20-22{ }^{\circ} \mathrm{C}, \\
\text { - } \mathrm{L}: \mathrm{D}(12: 12), 30 \mu \mathrm{E} \mathrm{m}^{-2} \mathrm{~s}^{-1}\end{array}$ & $\begin{array}{c}\text { Phycocolloid } \\
\text { yielding } \\
\text { seaweeds } \\
\text { (e.g., } \\
\text { Turbinaria } \\
\text { conoides) }\end{array}$ & $\begin{array}{l}\text { PES for red seaweeds or } \\
\text { modified PES with } 0.4 \% \\
\text { KI (PESI) for brown } \\
\text { seaweeds with } 1.5 \% \\
\text { Bacto agar }\end{array}$ & [166] \\
\hline & $\begin{array}{l}\text { - } \mathrm{T}: 15^{\circ} \mathrm{C} \\
\text { - } \mathrm{L}: \mathrm{D}(16: 8), 100 \mu \mathrm{E} \mathrm{m}^{-2} \mathrm{~s}^{-1}\end{array}$ & $\begin{array}{c}\text { Sargassum } \\
\text { heterophyllum }\end{array}$ & ASP-12 NTA & [167] \\
\hline & $\begin{array}{l}\text { - } \mathrm{T}: 15 \text { to } 25^{\circ} \mathrm{C} \\
\text { - L:D (12:12), 20-200 } \mu \mathrm{E} \mathrm{m}^{-2} \mathrm{~s}^{-1} \\
\text { (Blue light) }\end{array}$ & $\begin{array}{l}\text { Sargassum } \\
\text { horneri }\end{array}$ & $\begin{array}{c}\text { ESL medium } \\
\text { supplemented with } 5 \mu \mathrm{M} \\
\text { uniconazole (a triazole- } \\
\text { type inhibitor of } \\
\text { cytochrome } \mathrm{P}_{450} \text { enzymes) }\end{array}$ & [168] \\
\hline & $\begin{array}{l}\text { - } \mathrm{T}: 14{ }^{\circ} \mathrm{C}, \\
\text { - L:D (14:10), } 2000 \text { Lux, cool } \\
\text { white fluorescent lamps }\end{array}$ & $\begin{array}{l}\text { Medullary } \\
\text { tissues of } \\
\text { Laminaria } \\
\text { angustata }\end{array}$ & $\begin{array}{l}\text { ASP 12-NTA medium } \\
\text { solidified with } 1.0 \% \text { agar }\end{array}$ & [169] \\
\hline
\end{tabular}


Cont., Table 11: Some selected trials with callus cultures and plant regeneration in marine macroalgae organisms

\begin{tabular}{|c|c|c|c|c|}
\hline $\begin{array}{l}\text { Macroalagae } \\
\text { class }\end{array}$ & Growth parameters & Organism & Medium & Ref. \\
\hline \multirow[t]{4}{*}{$\begin{array}{l}\text { 2. Green and } \\
\text { Red algae }\end{array}$} & $\begin{array}{l}\text { - } \mathrm{T}: 18{ }^{\circ} \mathrm{C}, \\
\text { - } \mathrm{L}: \mathrm{D}(12: 12), 60 \mu \mathrm{E} \mathrm{m}^{-2} \mathrm{~s}^{-1}\end{array}$ & $\begin{array}{l}\text { Different } \\
\text { species of } \\
\text { green and red } \\
\text { macroalgae }\end{array}$ & $\begin{array}{l}\text { Seven different media } \\
\text { in liquid form and as } \\
\text { gels (e.g., sea water, } \\
\text { PES, ASP-C-1) (agar } \\
\quad 0.3-1.5 \% \text {, or } \\
\text { carrageenans } 0.5-3 \% \text { ). }\end{array}$ & [170] \\
\hline & $\begin{array}{l}\text { - } \mathrm{T}: 20^{\circ} \mathrm{C} \\
\text { - } \mathrm{L}: \mathrm{D}(12: 12), 10-20 \mu \mathrm{E} \mathrm{m}^{-2} \mathrm{~s}^{-1}\end{array}$ & $\begin{array}{l}\text { Some species } \\
\text { of red algae }\end{array}$ & $\begin{array}{c}\text { ASP-12 NTA solid } \\
\text { medium (1.5\% agar) } \\
\text { supplemented with IAA } \\
\text { and BAP }\end{array}$ & [171] \\
\hline & $\begin{array}{l}\text { For solid medium: } \\
\text { - } \mathrm{T}: 19^{\circ} \mathrm{C}, \\
\text { - } \mathrm{L}: \mathrm{D}(14: 10), 25 \mu \mathrm{E} \mathrm{m}^{-2} \mathrm{~s}^{-1} \\
\\
\quad \text { For liquid medium: } \\
\text { - } \mathrm{T}: 19^{\circ} \mathrm{C}, \\
\text { - } \mathrm{L}: \mathrm{D}(14: 10), 98 \mu \mathrm{E} \mathrm{m}^{-2} \mathrm{~s}^{-1}\end{array}$ & $\begin{array}{c}\text { Green algae } \\
\text { (Ulva pertusa) }\end{array}$ & $\begin{array}{l}\text { Artificial sea water } \\
\quad(0.7 \% \text { agar }) \\
\text { supplemented with } \\
\text { different plant growth } \\
\text { regulators, organic acids } \\
\text { and sugars }\end{array}$ & [172] \\
\hline & $\begin{array}{l}\text { - } \mathrm{T}: 20-22{ }^{\circ} \mathrm{C} \\
\text { - } \mathrm{L}: \mathrm{D}(12: 12), 5 \mu \mathrm{E} \mathrm{m}^{-2} \mathrm{~s}^{-1}\end{array}$ & $\begin{array}{c}\text { Red algae } \\
\text { (Gelidiella } \\
\text { acerosa) }\end{array}$ & $\begin{array}{l}\text { PES medium solidified } \\
\text { with } 1.5 \% \text { agar }\end{array}$ & [173] \\
\hline
\end{tabular}




\subsection{Materials and Methods}

3.2.1. Harvest and pre-treatment of algae

The brown algae $F$. vesiculosus was chosen also as a model for the brown macroalgae tissue culture development. Algae was harvested by Alfred Wegner Institute-Helmholtz Institute for Polar and Marine Research (Helgoland, Germany) from the North Sea in summer 2015. It was kept wet with sea water in ice-cooled boxes during transportation to the laboratory which was performed directly after collection.

As soon as algae had reached the laboratory, the thalli were washed thoroughly with tap water for 2-3 min removing visible symbionts with hands and brush, and then 1-2 min using sterile sea water or normal saline. Cleaned thalli were stored afterwards in wet hand tissues at $4{ }^{\circ} \mathrm{C}$.

\subsubsection{Surface sterilization}

All the following steps were performed in a laminar flow cabinet and with sterile reagents and materials. In addition, reagents were prepared in a sterile sea water. Thalli were cut into $7-10 \mathrm{~cm}$ pieces which divided afterwards into six groups to evaluate different surface sterilization protocols.

\section{a. Protocol 1: 2\% (w/v) Povidone iodine and 70\% $(v / v)$ ethanol}

Thallus pieces were dipped in 70\% (v/v) ethanol for 3-5 s, dried on filter paper, and washed with deionized water for $2 \mathrm{~min}$ to rinse the alcohol. Then, they were rubbed smoothly with Kleenex ${ }^{\circledR}$ for cleansing before being immersed in $2 \%(\mathrm{w} / \mathrm{v})$ povidone iodine solution for $4 \mathrm{~min}$. Povidone iodine was then removed with a filter paper and washed out for several times with deionized water for 2 $\min$.

\section{b. Protocol 2: 2\% (w/v) Povidone iodine, $70 \%$ ethanol and UV radiation}

The same steps were performed as in Protocol 1. Pieces were then cultured in MB50 and LB agar plates which further exposed to UV radiation for $15 \mathrm{~min}$.

c. Protocol 3: $2 \%(w / v)$ Povidone iodine, $70 \%$ ethanol, $10 \mu \mathrm{M} \mathrm{GeO}$ and 1\% (v/v) Triton X-100

The same steps were performed as in Protocol 1, except that $10 \mu \mathrm{M} \mathrm{GeO}_{2}$ was added to the povidone iodine solution, which was prepared in 1\% (v/v) Triton X-100.

\section{c. Protocol 4: Ultrasound, 5\% (w/v) povidone iodine, $10 \mu \mathrm{M} \mathrm{GeO}$ and antibiotics}

In the following Protocols (4-6), other mechanical and chemical treatments (e.g., ultrasound and antibiotics) were performed. The 7-10 cm pieces were thoroughly washed in PESA medium (PES 
medium without vitamins) three times ( 2 min each), and stored overnight in a fresh medium at $4{ }^{\circ} \mathrm{C}$. These pieces were then treated for $10 \mathrm{~s}$ in an ultrasound bath (40\%) at room temperature, and then rinsed once with water and once with medium. Pieces were then immersed in groups of 3-5 in a

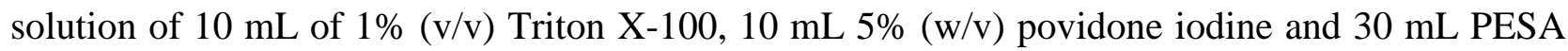
medium. The suspension was shaken vigorously with hand for $5 \mathrm{~min}$ and then treated in an ultrasound bath for $5 \mathrm{~s}$ again. To remove the brown colour of iodine, pieces were washed five times in a PESA medium. The next step utilized a concentrated antibiotic treatment. Thallus pieces were incubated for $1 \mathrm{~h}$ at $4{ }^{\circ} \mathrm{C}$ with $5 \mathrm{~mL} 1 \%$ (v/v) Triton X-100, $15 \mathrm{~mL} 30 \mathrm{x}$ filter-sterilized antibiotic mixture (constituents of antibiotic stock solution are described in Table 12) and $20 \mathrm{~mL}$ of PESA medium. Thallus pieces were washed several times with medium before a second antibiotic treatment with $5 \mathrm{~mL}$ of antibiotic stock solution in $45 \mathrm{~mL}$ PESA medium. This treatment was applied for $48 \mathrm{~h}$ without shaking in conditions similar to cultivation conditions (e.g., a photoincubator adjusted to $17{ }^{\circ} \mathrm{C}$, photoperiod of L:D (16:8) and $35 \mu \mathrm{E} \mathrm{m}^{-2} \mathrm{~s}^{-1}$ as a light intensity). Finally, pieces were thoroughly washed several times with PESA medium to remove antibiotic residuals before sterility and viability assessments.

Table 12: Antibiotic stock solution (30x) composition [274]

Antibiotics were used in Protocol 4-6.

\begin{tabular}{|l|l|}
\hline Component & Quantity $\mathbf{( m g )} / \mathbf{1 0 0} \mathbf{~ m L}$ \\
\hline Ampicillin sodium & 360.0 \\
\hline Kanamycin sulphate & 600.0 \\
\hline Tetracycline hydrochloride & 360.0 \\
\hline Chloramphenicol $* *$ & 300.0 \\
\hline Gentamycin sulphate & 240.0 \\
\hline Penicillin G sodium & 600.0 \\
\hline Erythromycin** & 600.0 \\
\hline Nystatin*** & 300.0 \\
\hline
\end{tabular}

* After preparation, the stock suspension was filter-sterilized and stored at $-20^{\circ} \mathrm{C}$.

** Prepared in $5 \mathrm{~mL}$ in $70 \%$ ethanol before addition to the stock solution.

*** Prepared in $5 \mathrm{~mL}$ in DMF before addition to the stock solution and used only in Protocol 6.

\section{d. Protocol 5: Ultrasound, 5\% (w/v) povidone iodine, $10 \mu \mathrm{M} \mathrm{GeO}$, antibiotics and UV radiation}

The same steps as Protocol 4 were performed, but cultured pieces were incubated in LB and MB50 media plates and then exposed to UV light for $15 \mathrm{~min}$. 
e. $\quad$ Protocol 6: Ultrasound, povidone iodine, $10 \mu \mathrm{M} \mathrm{GeO}_{2}$, antibiotics and Nystatin

Protocol 6 was performed similarly as Protocol 4, except that $300 \mathrm{mg}$ of Nystatin as an anti-fungal agent was mixed with the 30x antibiotic stock solution. Due to its poor water solubility of Nystatin, it was prepared in $5 \mathrm{~mL} \mathrm{DMF}$ before addition to the antibiotic stock solution. Performed processes of Protocols (1-6) could be summarized in Table 13.

\section{Table 13: Summary of processes performed in all surface sterilization protocols}

Steps are described after algae harvesting, washing with sterile sea water and cutting into $7-10 \mathrm{~cm}$ pieces.

\begin{tabular}{|c|c|c|c|c|c|c|}
\hline Type of treatment & $\begin{array}{c}\text { Protocol } \\
\mathbf{1}\end{array}$ & $\begin{array}{c}\text { Protocol } \\
\mathbf{2}\end{array}$ & $\begin{array}{c}\text { Protocol } \\
\mathbf{3}\end{array}$ & $\begin{array}{c}\text { Protocol } \\
\mathbf{4}\end{array}$ & $\begin{array}{c}\text { Protocol } \\
\mathbf{5}\end{array}$ & $\begin{array}{c}\text { Protocol } \\
\mathbf{6}\end{array}$ \\
\hline $\begin{array}{c}2 \% \text { (w/v) Povidone } \\
\text { iodine }\end{array}$ & + & + & + & - & - & - \\
\hline $70 \%(\mathrm{v} / \mathrm{v})$ ethanol & + & + & + & - & - & - \\
\hline UV radiation & - & + & - & - & + & - \\
\hline $\begin{array}{c}5 \% \text { (w/v) Povidone } \\
\text { iodine }\end{array}$ & - & - & - & + & + & + \\
\hline Ultrasound radiation & - & - & - & + & + & + \\
\hline $10 \mu \mathrm{M}$ GeO 2 & - & - & + & + & + & + \\
\hline $1 \%$ Triton X-100 & - & - & + & + & + & + \\
\hline $\begin{array}{c}\text { Antibiotic without } \\
\text { Nystatin }\end{array}$ & - & - & - & + & + & - \\
\hline $\begin{array}{c}\text { Antibiotic with } \\
\text { Nystatin }\end{array}$ & - & - & - & - & - & + \\
\hline
\end{tabular}

+: applied; -: not applied

\subsubsection{Sterility investigation}

After each protocol, treated thalli pieces were further cut into $2-3 \mathrm{~cm}$ pieces and cultured on MB50 and LB (Miller) solid media for sterility investigation. They were incubated in groups of 2-3 pieces for 2 weeks at $17{ }^{\circ} \mathrm{C}$ and $26^{\circ} \mathrm{C}$, respectively, in dark to check for any type of microbial growth. Incubation was performed in a static shaker incubator.

\subsubsection{Genotyping of microbial contaminants}

As described by AllPrep ${ }^{\circledR}$ DNA/RNA mini Kit (Qiagen, Germany) manual, genetic materials from contaminated explants were extracted and purified [174]. Samples were ground and put in sterile EMatrix-tubes (MP Biomedicals GmbH, Germany) which were filled with $600 \mu \mathrm{L}$ RLT lysis buffer 
and $6 \mu \mathrm{L}$ mercaptoethanol. Then, tissue and cell lysis was performed in a tissuelyzer for $2 \mathrm{~min}$ at 30 $\mathrm{Hz}$ and frozen at $-70{ }^{\circ} \mathrm{C}$ for $5 \mathrm{~min}$ in liquid nitrogen and repeated for three times. 18S SSU rRNA genes were identified and amplified from DNA and cDNA using the universal eukaryotic primers Euk-A (5'-AACCTGGTTGATCCTGCCAGT-3') and Euk-B (5'GATCCTTCTGCAGGTTCACCTAC-3') [175]. An initial denaturation at $98{ }^{\circ} \mathrm{C}$ for $30 \mathrm{~s}$, followed by 35 cycles at $98{ }^{\circ} \mathrm{C}$ for $10 \mathrm{~s}, 67^{\circ} \mathrm{C}$ for $30 \mathrm{~s}$ and $72{ }^{\circ} \mathrm{C}$ for $30 \mathrm{~s}$ and finally at $72{ }^{\circ} \mathrm{C}$ for 5 min were carried out. PCR products were checked by gel electrophoresis using 1\% (w/v) agarose and then purified by MinElute PCR Purification Kit (Qiagen, Germany). Afterwards, a nested PCR experiment was performed for the purified products using the Euk82F (5'GAAAGTCTGCTGAACTGGCTC-3') and Euk1517R (5'-ACGGCTACCTTGTTACGACTT-3') primers following the protocol which consisted of $98{ }^{\circ} \mathrm{C}$ for $30 \mathrm{~s}$ for denaturation, 30 cycles of 98 ${ }^{\circ} \mathrm{C}$ for $10 \mathrm{~s}, 53{ }^{\circ} \mathrm{C}$ for $30 \mathrm{~s}$, and $72{ }^{\circ} \mathrm{C}$ for $30 \mathrm{~s}$ and finally at $72{ }^{\circ} \mathrm{C}$ for $5 \mathrm{~min}$. Amplicons were checked and purified again as described before. Ligation and cloning of the PCR products were achieved as described in manual of NEB PCR cloning kit using pMiniT as a vector, before the isolated constructs were sent for sequencing by Seq-It GmbH (Kaiserslautern, Germany). Alignment of sequencing results were performed by NCBI-blast database in order to identify the microbial contamination.

3.2.5. Vitality investigation (2,3,5-Triphenyltetrazolium chloride (TTC) assay) According to a previously described protocol by Nam, et al. [176], 0.8\% (w/v) TTC was prepared in a $50 \mathrm{mM}$ Tris-HCl buffer ( $\mathrm{pH} 7)$ in PESA medium. Triplicates of $0.1 \mathrm{~g}$ pieces of treated as well as non-treated thalli were each incubated in $15 \mathrm{~mL}$ capped test tubes with $4 \mathrm{~mL}$ of TTC colorless solution and two drops of mineral oil. After $1 \mathrm{~h}$ incubation in the dark at $20{ }^{\circ} \mathrm{C}$ without shaking, thalli were washed with sterile PESA medium 3 times. To extract the red and water insoluble reduction product triphenylformazan (TPF) from tissues, $2 \mathrm{~mL}$ of $0.2 \mathrm{M} \mathrm{KOH}$ prepared in $25 \%(\mathrm{v} / \mathrm{v}$ ) ethanol was incubated with the thalli for $15 \mathrm{~min}$ in a drying oven at $60{ }^{\circ} \mathrm{C}$. For a quantitative determination of TPF, hexane $(2 \mathrm{~mL})$ was added to a cooled extract, the sample was then vortexed for $10 \mathrm{~s}$ and centrifuged (4500 rpm) for $1 \mathrm{~min}$. Finally, the upper red hexane layer was measured by colorimetry at $545 \mathrm{~nm}$, using hexane as a blank. 
3.2.6. Protoplast isolation and culture development

Protoplasts from $F$. vesiculosus were isolated and purified as described previously by Mussio and Rusig [177]. Briefly, $0.7 \mathrm{~g}$ of previously-sterilized explant with Protocol 6 was digested with $20 \mathrm{~mL}$ of a filter-sterilized enzyme solution (see Appendix C: Medium composition and preparations) for $20 \mathrm{~h}$ in the dark at room temperature in addition to $1 \mathrm{~h}$ in an overhead shaker (F1 mode, $30 \mathrm{rpm}$ ). After tissue digestion, the cell suspension was filtered by an autoclaved sieve with a $40 \mu \mathrm{m}$ mesh size. Isolated protoplasts were washed for two times in a sterile washing solution ( $5 \mathrm{~mL}$ each) containing $0.4 \mathrm{M} \mathrm{NaCl}$ and $5 \mathrm{mM} \mathrm{CaCl}_{2}$. A sample of isolated protoplasts was detected by UV light using $10 \mu \mathrm{g} \mathrm{mL}^{-1}$ Calcofluor white stain and a microscope.

3.2.7. Development of protoplast and callus-like cultures

Several growth variables were investigated to check their ability to induce callus and protoplast cultures either in solid, suspension cultures in addition to bioreactors. Cultures were incubated in a photoincubator at controlled parameters including temperatures and light regimes and intensities. Applied growth conditions were summarized, as shown in Fig. 39.

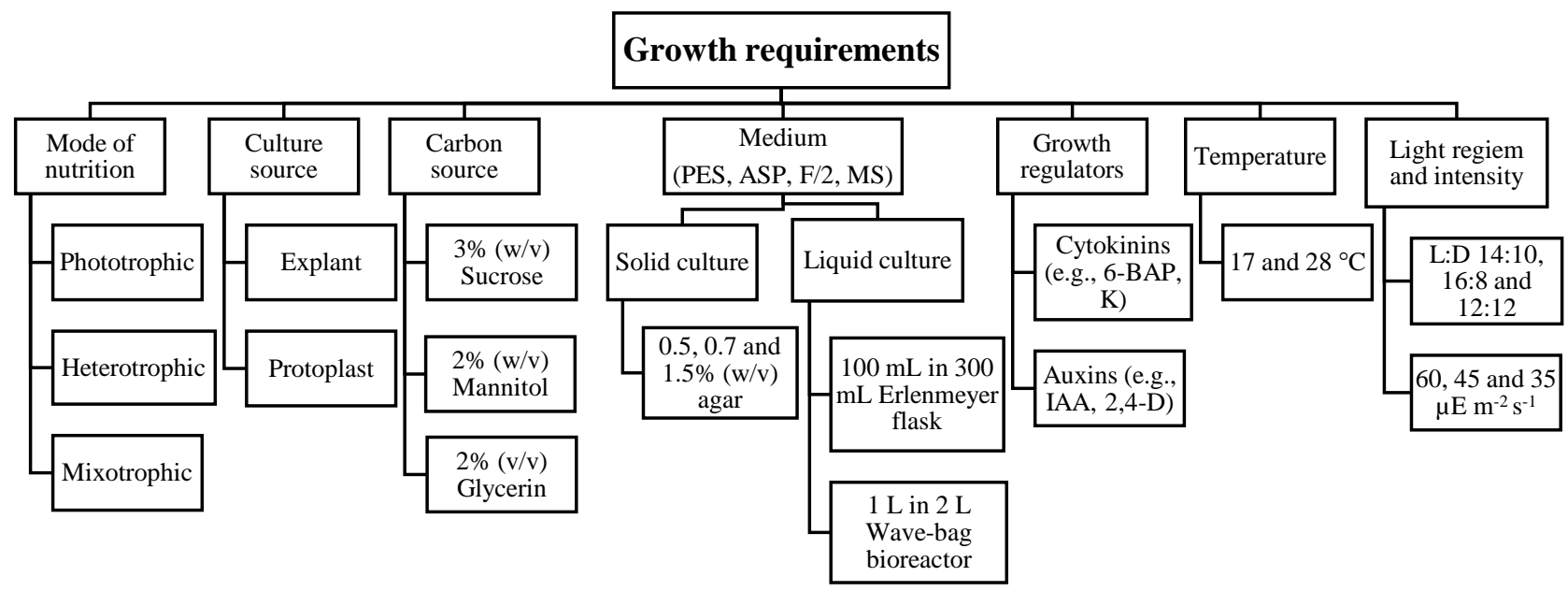

Fig. 39: Applied growth conditions and variables to induce callus-like and protoplast cultures These conditions were applied separately and in combinations during callus development. 
In more details, the different culture types were induced, as described in the following sections.

\subsubsection{Protoplast culture}

Induction of cell wall reformation and cell reproduction were performed as follows: $1 \mathrm{~mL}$ of washed isolated protoplasts was cultured in 2 steps, for one week as a heterotroph in a $50 \mathrm{~mL}$ protoplast medium containing $2 \mathrm{mM}$ HEPES, $1 \%$ (v/v) PES with $3.8 \%$ sea salt, $0.25 \mathrm{M}$ glucose, $0.15 \mathrm{M}$ sucrose, $0.025 \%(\mathrm{w} / \mathrm{v})$ casein hydrolysate, $20 \mu \mathrm{M}$ ornithine and supplemented with a combination of $45 \mu \mathrm{M} 2,4-\mathrm{D}$ and $40 \mu \mathrm{M}$ CPPU as plant growth regulators [178]. Afterwards, in a $50 \mathrm{~mL}$ PES medium, $10 \mathrm{~mL}$ of cultured protoplasts were sub-cultured as phototroph at $17{ }^{\circ} \mathrm{C}$ with a shaking rate (130 rpm) using an orbital shaker and L:D 14:10, $35 \mu \mathrm{E} \mathrm{m}^{-2} \mathrm{~s}^{-1}$. Mixotrophic and heterotrophic cultivation using $2 \%(\mathrm{v} / \mathrm{v})$ glycerin as a carbon source were also investigated. In parallel, cultivation in solid medium supplemented with $0.5 \%(\mathrm{w} / \mathrm{v})$ agar at the same previously conditions were applied.

\subsubsection{Callus induction}

a. Solid cultures

ASP-12-NTA, according to Provasoli [165], PES [155] and F/2 (Guillard's (F/2) Marine Enrichment medium purchased from Sigma-Aldrich ${ }^{\circledR} 50 \mathrm{x}$ stock solution) media supplemented with $0.5,0.7$ and $1.5 \%(\mathrm{w} / \mathrm{v})$ agar were incubated with 2-3 explants that were previously surface sterilized with Protocol 6. The cultures were cultivated as phototrophs, mixotrophs and heterotrophs in different light regimes including intensities at 60,45 and $35 \mu \mathrm{E} \mathrm{m}^{-2} \mathrm{~s}^{-1}$ and with photoperiods L:D of (16:8, 14:10 and $12: 12$ ) at $17^{\circ} \mathrm{C}$ and $28^{\circ} \mathrm{C}$. In mixotrophic and heterotrophic cultivation, either $3 \%(\mathrm{w} / \mathrm{v}$ ) sucrose or $2 \%(\mathrm{w} / \mathrm{v})$ mannitol or $2 \%(\mathrm{v} / \mathrm{v})$ glycerin were used as a carbon source.

In addition, different growth regulators, such as auxins (e.g., 2,4-D and IAA) and cytokinins (e.g., $\mathrm{K}$ and BAP) were supplemented some cultivation medium to study their effect on callus induction from explants. They were applied in concentrations of $0.1 \mathrm{mg} \mathrm{mL}^{-1}$ for IAA and BAP [171], $4 \mu \mathrm{M}$ for $2,4-\mathrm{D}$ and $2 \mu \mathrm{M}$ for $\mathrm{K}$.

\section{b. Liquid suspension cultures}

\section{i. Cultivation in Erlenmeyer flasks}

Applying the same conditions in solid cultures, two-three explant pieces were cultivated in $100 \mathrm{~mL}$ media in $300 \mathrm{~mL}$ Erlenmeyer flasks. The flasks were incubated for a week at static condition, then 
agitated at $130 \mathrm{rpm}$ using an orbital shaker. Sub-culturing was performed regularly at 6-8 weeks intervals.

\section{ii. $\quad$ Cultivation in wave bag photobioreactor $(P B R)$}

Rhythmic, wave-like movement of the wave bag bioreactor was attempted to induce callus growth directly from explants. In a $2 \mathrm{~L}$ bag, 20 algae explants of $F$. vesciculosus were incubated in phototrophic growth conditions in a $1 \mathrm{~L} \mathrm{PES} \mathrm{medium} \mathrm{at} 18{ }^{\circ} \mathrm{C}$ with a rocking speed $15 \mathrm{rmin}^{-1}$, a $10^{\circ}$ angel and an air flow rate of $90 \mathrm{~m} \cdot \mathrm{min}^{-1}$ with a photoperiod of L:D (16:8) and light intensity of 30-50 $\mu \mathrm{E} \mathrm{m}^{-2} \mathrm{~s}^{1}$. Regularly, $100 \mathrm{~mL}$ of medium were replaced weekly with a fresh medium under sterile conditions. 


\subsection{Results and Discussion}

3.3.1. Sterility investigations

\subsubsection{Surface sterilization procedures}

Complex interrelationships between marine organisms resulted in difficult complete removal of marine microbes and production of axenic cultures. Such features limit advances in in vitro marine biotechnology. Collection of clean and healthy algae from regions of low contamination or from deep water is recommended to ease the process of sterilization and obtain an axenic culture. Different treatments were investigated to produce axenic or unialgal cultures, for example:

a. 70\% (v/v) Ethanol had a harmful effect on microorganisms' cell wall leading to protein denaturation and precipitation. A major disadvantage of ethyl alcohol was its bleaching and nonspecific tissue destructive effect (i.e., immersion of explants for more than $10 \mathrm{~s}$ ) resulted in a pale green colored explant.

b. UV radiation had a germicidal effect. It penetrates microorganisms' cell wall and cytoplasmic membrane causing a molecular rearrangement of the microorganism's DNA that prevents it from reproducing.

c. $\mathbf{G e O}_{2}$ was applied to suppress the growth of diatoms. $\mathrm{GeO}_{2}$ competes with $\mathrm{SiO}_{2}$ in diatoms biochemical metabolic reactions. It was reported that the presence of $\mathrm{GeO}_{2}$ could inhibit growth of brown algae contrary to green and red algae [179]. Therefore, washing with sterilized sea water for several times was performed immediately after this step to remove Ge residuals.

d. Povidone iodine or Betadine ${ }^{\circledR}$ is a surface disinfectant against a broad spectrum of germs. Such treatment is well-known to establish axenic marine cultures [166,180].

e. Ultrasound radiation and Triton X-100 were effective tools to disintegrate and dislodge associated adherent microorganisms [181]

f. Broad-spectrum antibiotics with bactericidal and bacteriostatic effect were applied in two steps as the main tool to remove marine bacteria including cyanobacterial contamination $[181,182]$. Because the nature of marine bacterial flora is variable and not well-characterized, a mixture of broad-spectrum antibiotics was effectively applied.

g. Anti-fungal agent like Nystatin was used to control the fungal contamination [154].

Results showed that cultured explants sterilized by all protocols, except Protocol 6, suffered from contamination in all cultured plates in LB and MB50 plates. The best results were obtained from Protocol 6, which showed that more than $90 \%$ of cultivated explants were sterile. In Protocol 6, the 
cultured explants on MB50 and LB media still showed contamination until the second treatment of antibiotics, as reported in Fig. 40.

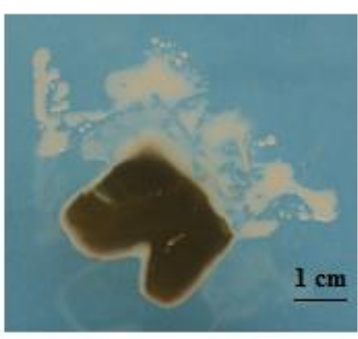

a

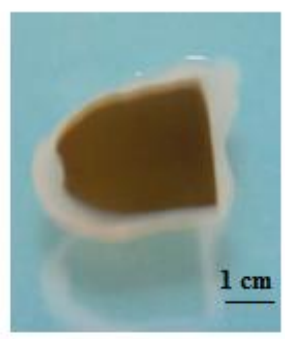

b

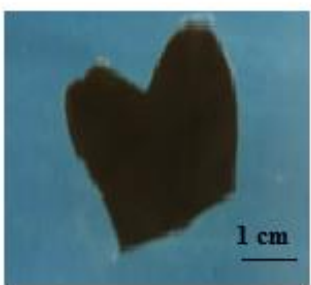

e

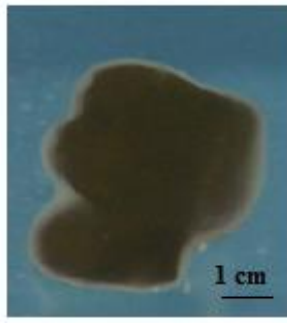

c

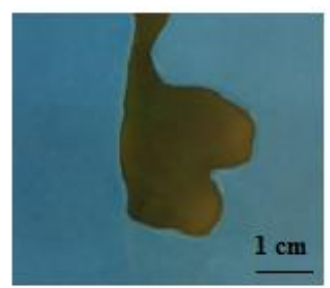

f

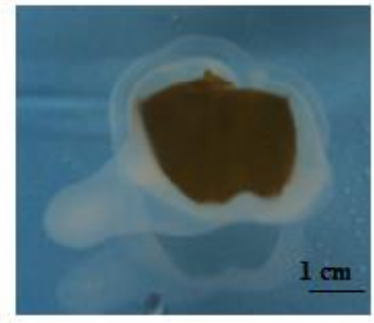

d

Fig. 40: Different explants of $F$. vesiculosus incubated on $\mathrm{MB50}(\mathrm{a}, \mathrm{b}, \mathrm{c}, \mathrm{d}$ and e) and $\mathrm{LB}$ (f) media at $17^{\circ} \mathrm{C}$ and $26{ }^{\circ} \mathrm{C}$, respectively, after different steps of Protocol 6

a: non-treated explant; b: after washing with sterilized PESA medium step; c: after sonication step; d: after treatment with povidone iodine and Triton X-100; $\mathbf{e}$ : after a $2^{\text {nd }}$ treatment with broad-spectrum antibiotics including Nystatin; $\mathbf{f}$ : after a $2^{\text {nd }}$ treatment step with antibiotics including Nystatin on LB medium.

\subsubsection{Genotyping of microbial contaminants}

Results of $18 \mathrm{~S}$ rRNA nucleotide sequencing which was isolated from contaminated explants revealed the presence of Aplanochytrium sp. with an identity of 97\%. Aplanochytrium sp. (Labryinothulomycota) are marine heterotrophic stramenopiles that associate often in a parasitic relationship with dead and decay marine algae [183]. These findings showed the importance of the addition of an anti-fungal agent during surface sterilization procedures.

\subsubsection{Vitality investigation}

Enzymatic reduction of TTC was investigated to determine quantitatively axenic explants vitality and their metabolic activity, in comparison with non-sterilized thallus tissues. TTC is a colourless, water-soluble reagent that enzymatically-transformed to red, water-insoluble TPF through a 
reduction reaction [184]. This biotransformation reaction occurred through a tetrazolium radicle, as shown in Fig. 41, and thus tissue dehydrogenase activity and vitality could be quantitatively determined. The enzymatic conversion was evaluated by absorbance measurement at $545 \mathrm{~nm}$ to prevent interferences from other pigments [185]. Since TTC is oxygen- [186] and light-sensitive [184], mineral oil was added to cover the reaction surface without shaking and performed in darkness, respectively.

In optimized protocol (i.e., Protocol 6), tissue vitality (\%) after the washing step with sterilized sea water or PESA medium, after ultrasound treatment and by the end of the protocol recorded $78 \pm 1.01$, $51 \pm 1.13$ and $29 \pm 0.026 \%$, respectively, relative to vitality of a non-sterile thallus. Before complete axenic conditions, marine microbes interfered also with this reaction results, because they had also the ability to reduce TTC. Therefore, by the end of protocol, the explants were only responsible for the $29 \%$ vitality detected during the TTC conversion to TPF.

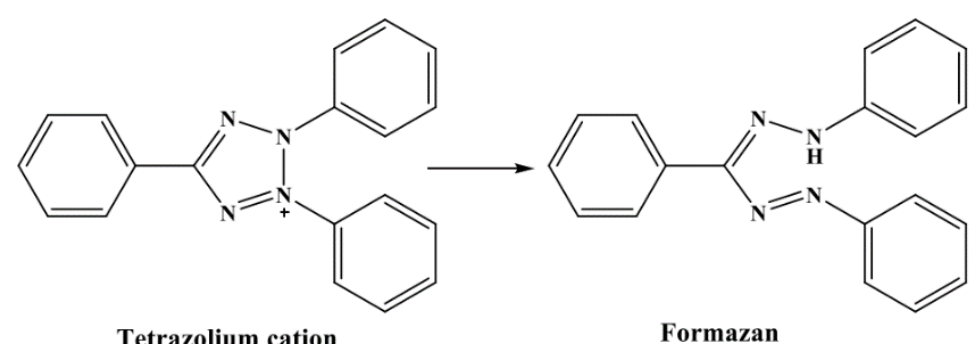

Fig. 41: Enzymatic reduction of tetrazolium chloride (TTC) (Tetrazolium cation, colorless) to triphenylformazan (TPF) (Formazan, red color)

3.3.3. Protoplasts isolation and culture development

After protoplasts isolation from $F$. vesiculosus axenic explant, protoplasts protected themselves from destruction [156] and were elicited to reform their cell walls and then reproduce normally. This was performed by incubation of them in the protoplast cultivation medium which was a highly osmotic medium. Therefore, protoplast cultivation medium was supplemented with $250 \mathrm{mM}$ glucose, $150 \mathrm{mM}$ sucrose and 3.8\% (w/v) sea salts, that increased medium osmotic pressure. As Fig. 42 demonstrates, protoplasts after isolation were scattered irregularly without any specific arrangement, but upon culturing, they begun to reproduce with clumps or aggregates formation. 


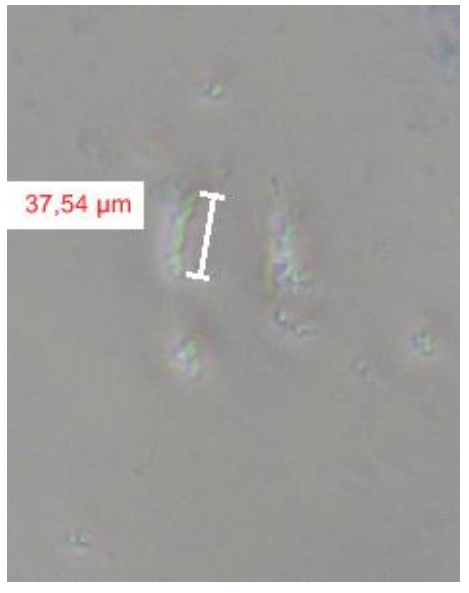

Fig. 42: Protoplasts after $72 \mathrm{~h}$ heterotroph cultivation in the protoplast medium showing start of cell multiplication and aggregation

Isolated protoplasts were identified by UV light with the aid of Calcofluor white stain [177] (not shown). However, cell aggregates were detected normally using white light.

The results were highly promising for the transfer of such reformed cells to specific PES solid medium with $0.5 \%(\mathrm{w} / \mathrm{v})$ agar and liquid marine media supplemented with $2 \%(\mathrm{v} / \mathrm{v})$ glycerin to develop either mixotrophic or heterotrophic protoplast cultures of $F$. vesiculosus. Unfortunately, cells stopped their reproduction with sub-culturing.

3.3.4. Callus-like development from $F$. vesiculosus explant

\subsubsection{Solid medium}

Another strategy to develop a callus-like growth from brown seaweeds was carried out. Different combinations of nutrition mode, media, light regime and phytohormones were applied. A phototrophic callus-like structure was successfully induced on ASP-12-NTA medium supplemented with $0.5 \%(w / v)$ agar using a light regime of L:D (14:10), $35 \mu \mathrm{E} \mathrm{m}^{-2} \mathrm{~s}^{-1}$ at $17{ }^{\circ} \mathrm{C}$ after five weeks of cultivation. The callus showed colourless with friable to filamentous-like cell mass, as demonstrated in Fig. 43. The callus was sub-cultured afterwards regularly every six-to-eight weeks on the same medium composition solidified with $0.7 \%(\mathrm{w} / \mathrm{v})$ agar. 


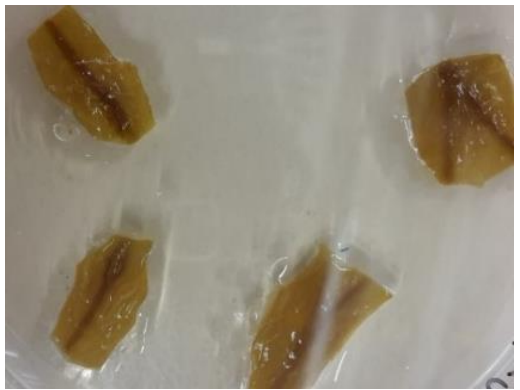

a

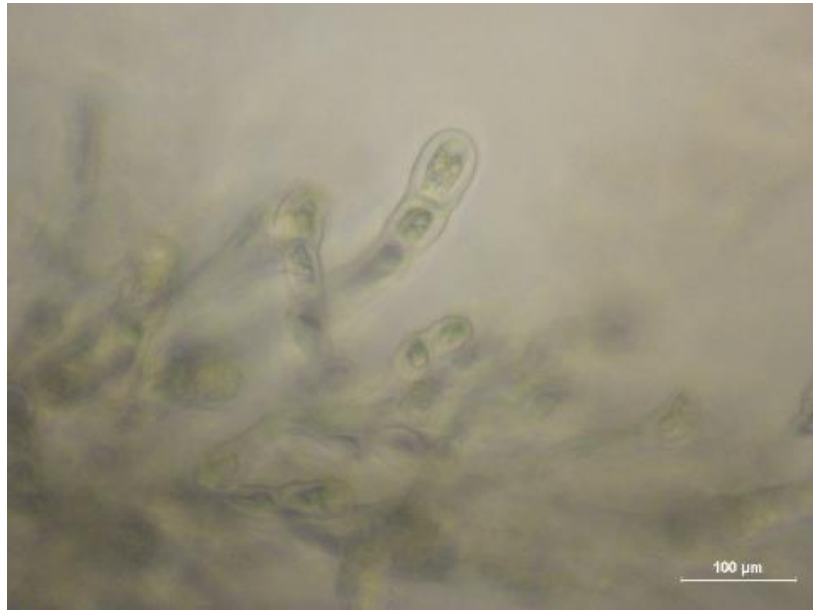

b

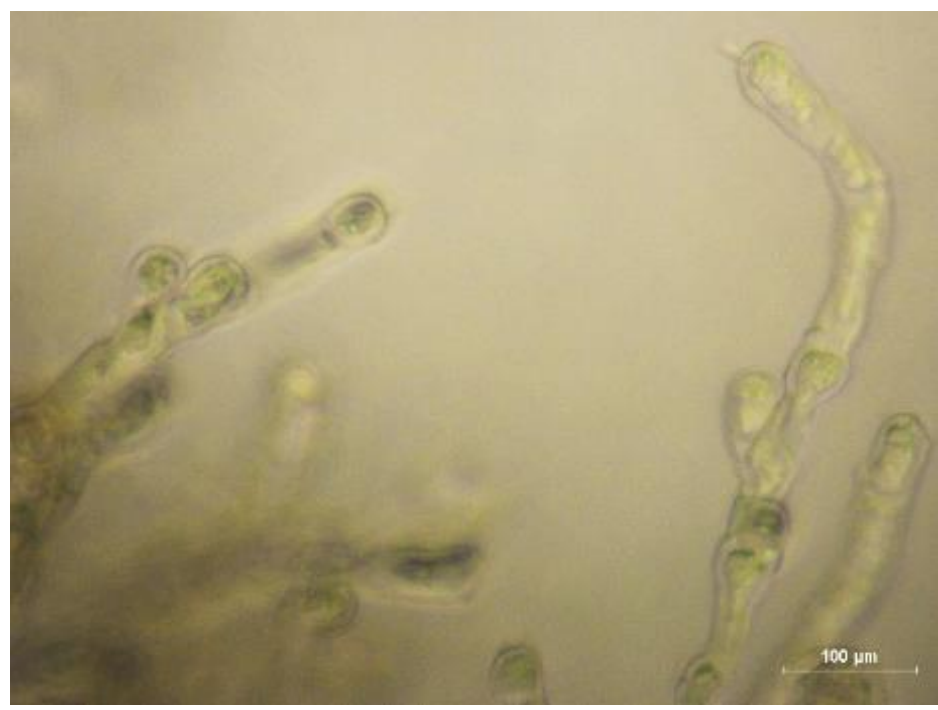

C

Fig. 43: Development of a phototrophic callus-like growth from $F$. vesiculosus explant in ASP-12-NTA medium

(a) Axenic F. vesiculosus explants in ASP-12-NTA medium supplemented with $0.5 \%(\mathrm{w} / \mathrm{v})$ agar. Phototrophic cultivation after three weeks led to the disappearance of the explant greenish colour. After five weeks of a phototroph cultivation applying L:D (14:10) light regime, $35 \mu \mathrm{E} \mathrm{m} \mathrm{m}^{-2} \mathrm{~s}^{-1}$ at a temperature of $17{ }^{\circ} \mathrm{C}$, a callus-like growth was developed and sub-cultured on the same medium supplemented with $0.7 \%(w / v)$ agar. (b,c) Filamentous callus-like growth after six months of subculturing developed into a plantlet-like growth.

\subsubsection{Cultivation in liquid suspension cultures}

Induction of callus-like growth in normal shaking flasks applying different conditions was not possible. Fortunately, cultivation in a wave bag-mixed bioreactor successfully utilized its advantage of rhythmic wave-like movement and produced a callus-like growth in a shorter cultivation time than classical techniques. After four weeks in a PES medium of phototrophic cultivation, explants 
of $F$. vesiculosus showed a filamentous, callus-like growth similar to that developed on solid medium, as shown in Fig. 44 (b). Application of a wave bag bioreactor in the field of seaweeds tissue culture was used for the first time. It is often used in cultivation of animal, insect and plant cell lines [145].

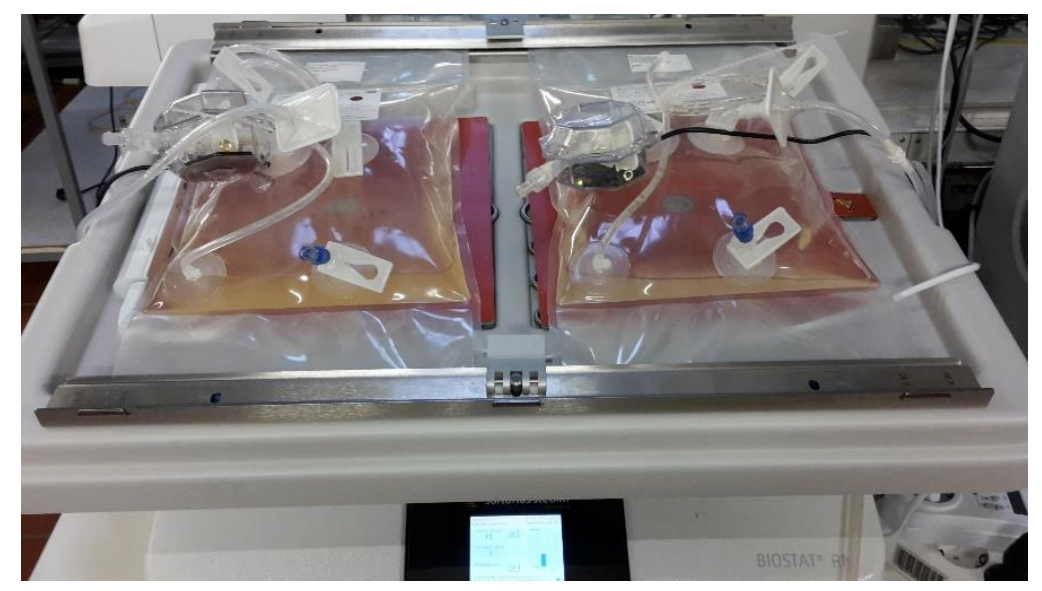

$\mathbf{a}$

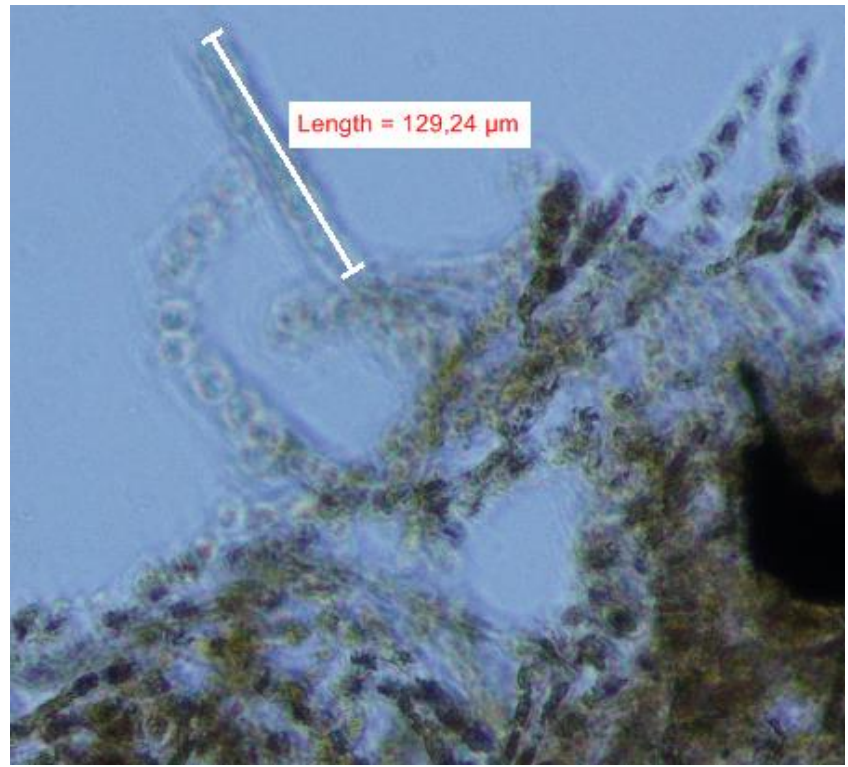

b

Fig. 44: (a) Cultivation of $F$. vesiculosus explants in a wave bag bioreactor, (b) Filamentous callus-like growth from $F$. vesiculosus explant developed after four weeks of phototrophic cultivation in PES medium 


\subsection{Conclusion and Prospectives}

Development of axenic protoplast and callus-like phototrophic cultures were possible. A time-saving and efficient surface sterilization protocol was established for marine macroalgae in only three days. The protocol combined different mechanical and chemical treatments, instead of ineffective separately-applied sterilization techniques. In comparison with different sterilization protocols, results showed that more than $90 \%$ of cultivated explants demonstrated unialgal cultures, when an anti-fungal agent was added to the broad spectra antibiotics. Moreover, the optimized protocol did not greatly affect the explant viability and had $29 \%$ vitality relative to a non-sterile tissue.

Furthermore, induction of protoplast, callus-like were performed either in a solid medium or a wave bag bioreactor from brown macroalgae. Moreover, in comparison with terrestrial plants, phytohormones did not have a significant effect on callus induction confirming its debatably role in marine biotechnology [145]. Interestingly, algal farming through tissue culture in a wave bag bioreactor was applied for the first time in marine biotechnology. Unfortunately, maintenance of callus-like and protoplasts cultures sterile and undefined nutrition requirements were limiting factors to continue their further growing and durability.

Future work should focus on maintenance of cultures' axenic conditions and defining of nutritional requirements to obtain a high-weighed biomass that could be induced to produce their secondary metabolites including fucoidan. Different variables including dehydration stress and cultivation using warm conditions might induce fucoidan production as a trial preventing algae desiccation [74]. In addition, development of a fast growing hairy root system has not been known or performed yet in marine biotechnology of brown macroalgae. However, transformation of tissues from terrestrial plants with the "natural genetic engineer" Agrobacterium rhizogenes, has been successfully performed for more than three decades as a tool for plant cultivation. So far, hairy root cultures have been developed from more than 100 plant species, including several endangered medicinal plants, giving opportunities to produce important phytochemicals in environment-friendly conditions [187]. In nature ecosystem, wounded plants secrete simple phenolic compounds, such as acetosyringone, which induce the plasmid-localized vir (virulence) genes of A. tumefaciens transferring the T-DNA fragments of the $\mathrm{Ti}$-(tumor inducing) plasmid to plant cells. This transfer results in a rapidly-dividing tumor cell or hairy-root-like growth [188]. 
Interestingly, it was reported that photosynthetic dinoflagellates (e.g. Symbiodinium sp.) were susceptible to be transformed by A. tumefaciens [189]. This was a motivation to start transfection of $F$. vesiculosus explant with $A$. tumefaciens and development of a hairy-root culture.

\subsubsection{Crown gall-like growth development in F. vesiculosus}

Rhizopium radiobacter (previously, Agrobacterium tumefaciens DSM 30147) was ordered from the Leibniz Institute DSMZ-German Collection of Microorganisms and Cell Cultures. The lyophilized bacterial strain was then regenerated and cultured on nutrient agar medium at $30^{\circ} \mathrm{C}$ for two days, as described by the supplier's protocol. A liquid culture was afterwards prepared from a bacterial colony previously grown on solid medium.

As mentioned before by Georgiev, et. al. in hairy-root induction in terrestrial plants [187], previously two-to-three wounded aseptic explants of $F$. vesiculosus were then transfected by incubation with $R$. radiobacter in a nutrient liquid, and then solid media with $1.5 \%(\mathrm{w} / \mathrm{v})$ agar each of $72 \mathrm{~h}$ at $30{ }^{\circ} \mathrm{C}$. Transgenic explants were, afterwards, transferred to an agar medium supplemented with $1 \mathrm{x}$ antibiotic solution for another $72 \mathrm{~h}$. Sterile transformed tissues were then cultivate as phototroph using PES, Guillard's F/2 and ASP-12 NTA supplemented with 0.7\% (w/v) agar.

As shown in Fig. 45, a crown gall or a hairy-root disease was detected in transformed $F$. vesiculosus tissues after six days of incubation of wounded explants with A. tumefaciens in a nutrient liquid and agar media each of three days. 


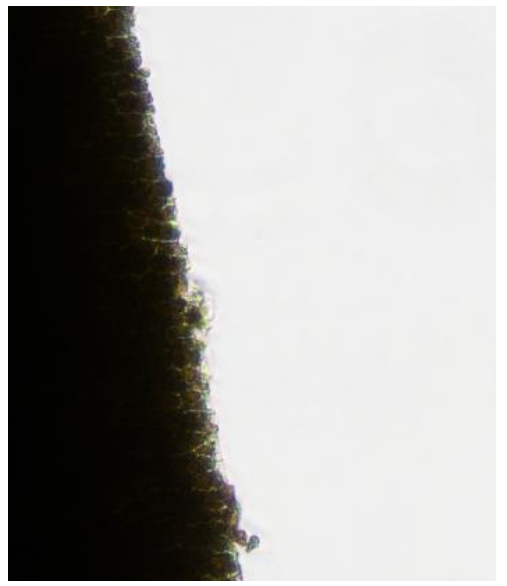

$\mathbf{a}$

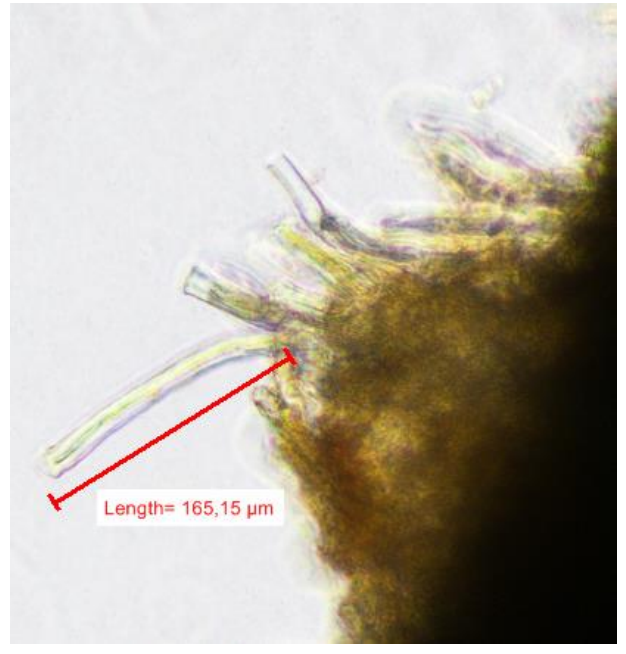

b

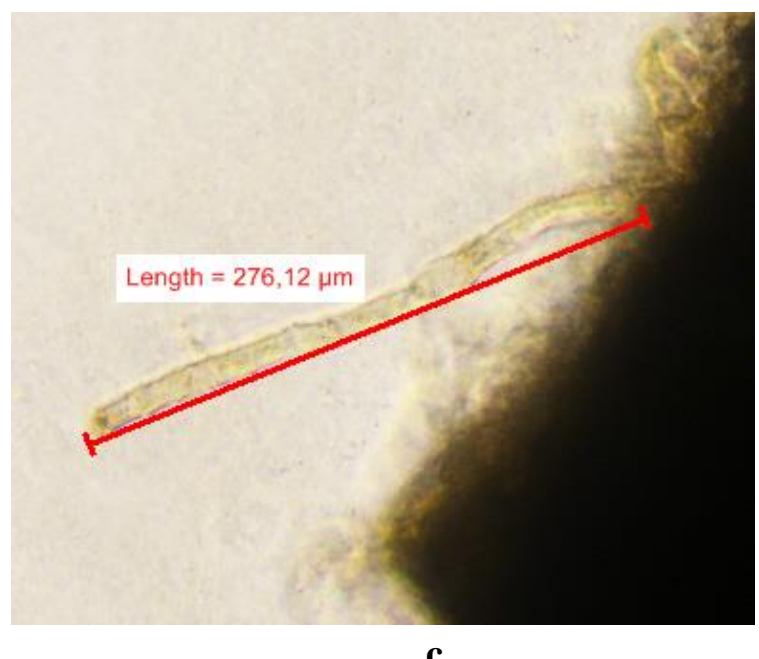

c

Fig. 45: Development of crown gall or hairy-root in $F$. vesiculosus explants after transfection with $R$. radiobacter or A. tumefaciens DSM 30147

(a): Non-infected intact smooth surface of $F$. vesiculosus; (b and c): Developed crown gall and hairyroot disease from a wounded explant after incubation with $R$. radiobacter liquid and agar nutrient media each of three days.

Unfortunately, excised hairy-root growth was deteriorated and not able to accommodate after phototrophic sub-culturing in PES, Guillard's F/2 and ASP-12 NTA solid media. In addition, transfer of $T i$-plasmid to algal cells has not been confirmed genetically yet. Further experiments should be also performed to investigate the required cultivation conditions for accommodation and growth of the newly-born crown gall-like growth from F. vesiculosus. Moreover, genomic DNA should be identified to confirm the presence of the Ti-plasmid of $R$. radiobacter in infected tissues [190]. 



\section{Heterologous Expression of Enzymes Involved in Fucoidan Biosynthesis}

\subsection{Introduction}

Problematic cultivation of brown seaweeds through tissue culture discussed previously in Chapter $\mathbf{3}$, in addition to structure heterogeneity of extracted fucoidan discussed in Chapter $\mathbf{2}$ created a novel strategy to produce an engineered fucoidan by its enzymatic synthesis. Enzymatic synthesis of a GMP-compliant fucoidan is a novel technique that has not been discussed in literature yet. An advantage for this strategy is a product that can be easily manipulated without being affected by algae species, extraction method, seasonal and geographical variations. Systematic chemical synthesis of low molecular weight fucoidan was performed successfully [191]; nevertheless specific synthesis by regio-specific and stereo-selective enzymes is still a challenge to provide a high-quality, consistent and long chain product in a short and time saving protocol.

Recent extensive bioinformatics and phylogenetic analyses have been performed in the brown macroalgae Ectocarpus siliculosus. They succeeded to reveal a lot of biosynthetic and remodeling information concerning the extracellular matrix (ECM) polysaccharides including fucoidan, cellulose and alginate [192]. Furthermore, performed genome analysis revealed the genes encode for enzymes that involved in fucoidan biosynthesis.

Fucoidan, as a high molecular weight homo- or hetero- sulphated polysaccharides, several enzymes contribute to its biosynthetic pathway. As shown in Fig. 46, it was postulated that fucoidan or sulphated fucan is synthesized in brown algae from the precursor GDP-L-fucose, which is obtained either by a de novo pathway from GDP-mannose or a salvage pathway from L-fucose. De novo synthesis of GDP-L-fucose is carried out in 2 steps catalyzed by GDP-mannose 4,6-dehydratase (GM46D) (1) and a bifunctional GDP-L-fucose synthetase (GFS) or GDP-4-keto-6-deoxy-Dmannose epimerase-reductase (2). However, salvage pathway is minor and catalyzed by the bifunctional l-fucokinase (FK) and GDP-fucose pyrophosphorylase (GFPP) with an aid from the GHMP kinase [192] from cytosolic L-fucose. GDP-L-fucose is further polymerized and elongated by different fucosyltransferases (FucTs) related to Glycosyltransferases families GT10, 23 and 65 to fucan polymer (5). The polymer is afterwards sulphated in a specific pattern by another group of enzymes; namely carbohydrate sulphotransferases (STs) to the final product sulphated fucan or fucoidan (6). 


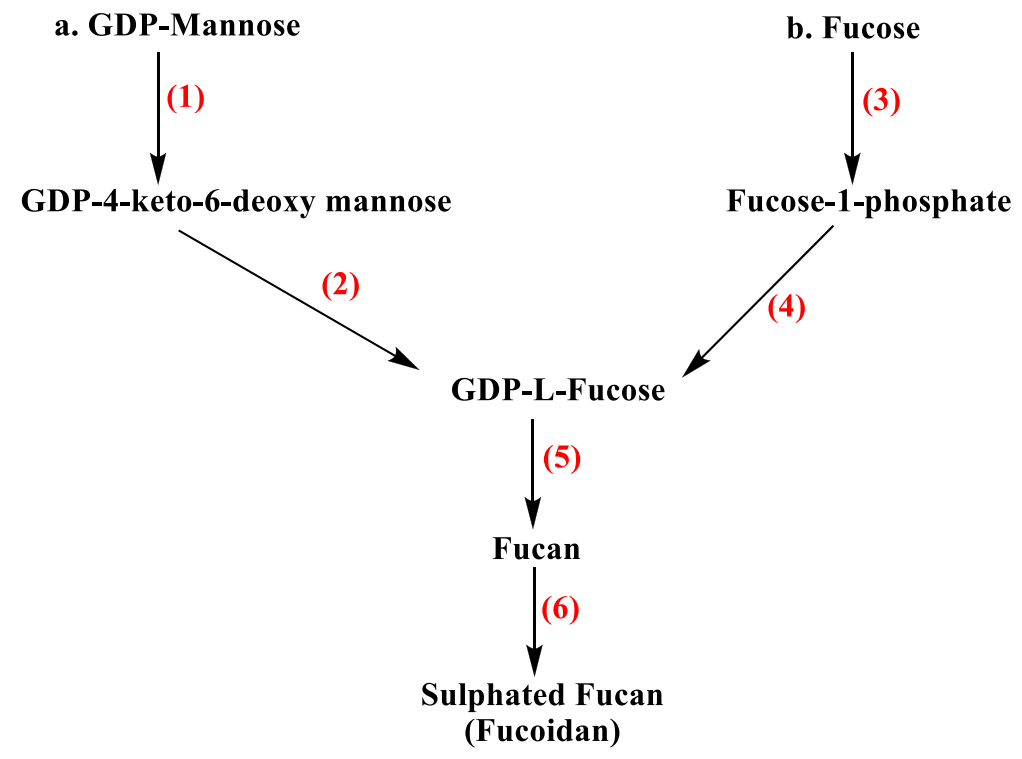

Fig. 46: The two different possible pathways for fucoidan biosynthesis in the brown algae Ectocarpus siliculosus (a) De novo pathway and b) salvage pathway

1) GDP-mannose 4,6-dehydratase (GM46D); 2) GDP-L-Fucose synthetase (GFS) or GDP-4-keto6-deoxy-D-mannose epimerase-reductase (GMER); 3) L-Fucokinase (FK); 4) GDP-fucose pyrophosphorylase (GFPP); 5) Fucosyltransferases (FuTs); 6) Sulphotransferases (STs). Modified after Michel, G. et al. [192].

In more details, Fig. 47 illustrates the de novo and salvage pathways for GDP-L-fucose synthesis from GDP-mannose and L-fucose, respectively, according to Yan Ren, et. al., in the filamentous fungus Mortierella alpina [193].

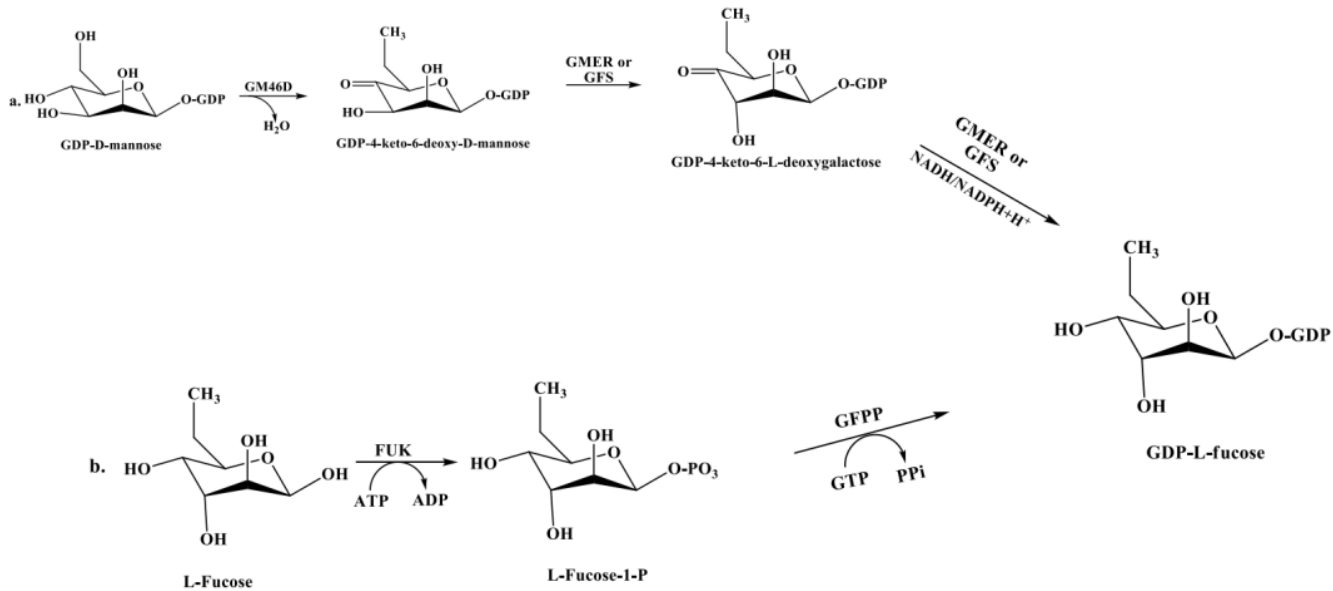

Fig. 47: Detailed de novo (a) and salvage pathway (b) for GDP-L-fucose biosynthesis either from GDPmannose or cytosolic L-fucose, respectively (modified according to [193]) 
Alternatively, heterologous expression of such enzymes in fast growing cell lines such as bacteria, yeast or even mammalian cell lines could be a potential technique for fucoidan production. Enzymes with similar functions were successfully over-expressed in bacteria and other cell lines which encouraged performing and starting similar experiments in the production cell factory E. coli. Table 14 summarizes some of the previously-performed heterologous expression experiments of similar enzymes from different biogenic sources in bacteria, yeast and mammalian cell lines.

Table 14: Examples of previous trials with heterologous expression to overexpress similar enzymes from different resources to that involved in fucoidan biosynthesis

\begin{tabular}{|c|c|c|c|c|}
\hline $\begin{array}{l}\text { Name of the } \\
\text { enzyme }\end{array}$ & Name of gene & Source & Expression system & Ref. \\
\hline \multicolumn{5}{|c|}{ a. de novo GDP-L-Fucose synthesis } \\
\hline \multirow[t]{11}{*}{$\begin{array}{c}\text { GM46D and GFS } \\
\text { or GMER }\end{array}$} & cDNA & $\begin{array}{l}\text { Mortierella } \\
\text { alpine }\end{array}$ & E. coli BL21 (DE3) & [193] \\
\hline & gmd (GM46D) and & E. coli K-12 & S. cerevisiae & [194] \\
\hline & $w c a G$ (GFS or GMER) & & \multirow[t]{2}{*}{ E. coli BL21(DE3) } & [195] \\
\hline & $\begin{array}{c}\text { BT_1224 (GM46D) }_{\text {and }} \text { BT_1225 (GFS or }_{-} \\
\text {GMER) }\end{array}$ & $\begin{array}{c}\text { Bacteroides } \\
\text { thetaiotaomicron }\end{array}$ & & [196] \\
\hline & gmd & E. coli (INVF1) & E. coli & [197] \\
\hline & Human cDNA & Human & $\begin{array}{c}\text { Defective Lec13, a CHO } \\
\text { cell line deficient in } \\
\text { GM46D activity }\end{array}$ & [198] \\
\hline & $b c e N$ & $\begin{array}{c}\text { Burkholderia } \\
\text { cenocepacia } \\
\text { J2315 }\end{array}$ & E. coli BL21 (DE3) & [199] \\
\hline & cDNA & Homo sapiens & $\begin{array}{c}\text { human hepatocarcinoma } \\
\text { cell line HepG2 }\end{array}$ & [200] \\
\hline & HP0044 and HP0045 & $\begin{array}{l}\text { Helicobacter } \\
\text { pylori }\end{array}$ & E. coli BL21 (DE3) & [201] \\
\hline & MURl (GM46D) & $\begin{array}{c}\text { Arabidopsis } \\
\text { thaliana }\end{array}$ & E. coli & $\begin{array}{l}202] \\
{[203]}\end{array}$ \\
\hline & $\begin{array}{l}\text { A118R (GM46D) and } \\
\text { A295L (GMER) }\end{array}$ & $\begin{array}{c}\text { Paramecium } \\
\text { bursaria } \\
\text { Chlorella } \text { Virus } 1 \\
\text { (PBCV-1) }\end{array}$ & E. coli $\mathrm{K} 803$ & [204] \\
\hline
\end{tabular}


Cont., Table 14: Examples of previous trials with heterologous expression to overexpress similar enzymes from different resources to that involved in fucoidan biosynthesis

\begin{tabular}{|c|c|c|c|c|}
\hline $\begin{array}{l}\text { Name of the } \\
\text { enzyme }\end{array}$ & Name of gene & Source & Expression system & Ref. \\
\hline \multicolumn{5}{|c|}{ b. Salvage GDP-L-fucose synthesis } \\
\hline \multirow[t]{5}{*}{ FK and GMER } & $f k / g f p p$ & \multirow{2}{*}{$\begin{array}{l}\text { Bacteroides } \\
\text { fragilis }\end{array}$} & S. cerevisiae & [205] \\
\hline & $f k p$ & & E. coli BL21(DE3) & $\begin{array}{l}{[206]} \\
{[207]}\end{array}$ \\
\hline & $A t F K G P$ & A. thaliana & E. coli BL21(DE3) & [208] \\
\hline & $F U K$ & \multirow{2}{*}{ M. alpina } & E. coli & [209] \\
\hline & GFPP & & E. coli $\mathrm{BL} 21(\mathrm{DE} 3)$ & [210] \\
\hline \multicolumn{5}{|c|}{ Fucosyltransferases (FucTs) } \\
\hline \multirow{5}{*}{$\begin{array}{c}\alpha 1,3- \\
\text { fucosyltransferase }\end{array}$} & HpfucT & \multirow[t]{2}{*}{ H. pylori } & E. coli $\mathrm{CSRDE} 3$ & [211] \\
\hline & fucT $(H P 0379)$ & & E. coli & [212] \\
\hline & human $(1,3 / 4)(F U T 3)$ & $\begin{array}{c}\text { Human } F U T 3 \\
\text { cDNA }\end{array}$ & Pichia pastoris & [213] \\
\hline & FucT & $\begin{array}{l}\text { Maize (Zea } \\
\text { mays) }\end{array}$ & $\begin{array}{c}\text { Spodoptera frugiperda } \\
\text { Sf21 cells by baculovirus } \\
\text { mediated infection }\end{array}$ & [214] \\
\hline & fucT VI & Human & Pichia pastoris & [215] \\
\hline $\begin{array}{c}\alpha 1,6- \\
\text { fucosyltransferase }\end{array}$ & $\operatorname{nodZ}$ & $\begin{array}{l}\text { Rhizobium sp. } \\
\text { NGR234 }\end{array}$ & E. coli XL1-Blue MRF' & [216] \\
\hline \multirow{8}{*}{$\begin{array}{c}\alpha 1,2- \\
\text { fucosyltransferase }\end{array}$} & WbgL & E. coli $\mathrm{O} 126$ & \multirow[t]{4}{*}{ E. coli BL21(DE3) } & [206] \\
\hline & WbsJ & E. coli $\mathrm{O} 128$ & & [217] \\
\hline & $w b n K$ and $w b w K$ & E. coli $\mathrm{O} 86$ & & [218] \\
\hline & wbiQ & E. coli $\mathrm{O} 127$ & & [219] \\
\hline & \multirow[t]{2}{*}{ fucT2 } & $\begin{array}{c}\text { H. pylori NCTC } \\
364\end{array}$ & E. coli JM 109 (DE3) & [220] \\
\hline & & H. pylori UA802 & E. coli CLM4 (pGP1-2) & [221] \\
\hline & futC & $\begin{array}{c}\text { H. pylori } \\
\text { NCTC11639 }\end{array}$ & E. coli BL21 (DE3) & [222] \\
\hline & $w c f B$ & $\begin{array}{c}\text { Bacteroides } \\
\text { fragilis }\end{array}$ & E. coli BL21star (DE3) & [223] \\
\hline \multicolumn{5}{|c|}{ Sulphotransferases (STs) } \\
\hline \multirow{3}{*}{$\begin{array}{c}\text { Arylsulphate } \\
\text { sulphotransferase } \\
\text { (ASST) }\end{array}$} & HAST1 and HAST3 & Human cDNA & E. coli $\mathrm{DH} 5 \alpha \mathrm{F}^{\prime} \mathrm{IQ}^{\mathrm{TM}}$ & [224] \\
\hline & ast $A$ & Klebsiella $\mathrm{K}-36$ & \multirow[t]{2}{*}{ E. coli BL21 (DE3) } & [225] \\
\hline & Hoch_5094 & $\begin{array}{c}\text { Haliangium } \\
\text { ochraceum }\end{array}$ & & [226] \\
\hline
\end{tabular}


Cont., Table 14: Examples of previous trials with heterologous expression to overexpress similar enzymes from different resources to that involved in fucoidan biosynthesis

\begin{tabular}{|c|c|c|c|c|}
\hline $\begin{array}{c}\text { Name of the } \\
\text { enzyme }\end{array}$ & Name of gene & Source & Expression system & Ref. \\
\hline \multirow{4}{*}{$\begin{array}{c}\text { Heparan sulfate } \\
O \text { - } \\
\text { sulphotransferase } \\
\text { (OST) }\end{array}$} & $\begin{array}{l}\text { A truncate version } \\
\text { (human } r-3-O S T 1 \text { ) }\end{array}$ & \multirow[t]{2}{*}{ Human cDNA } & & [227] \\
\hline & $\begin{array}{c}N S T, 2-O S T, 3-O S T-1, \\
6-O S T-1 \text { and 6-OST-3 }\end{array}$ & & $\begin{array}{c}\text { Yeast cells } \\
\text { (Kluyveromyces lactis) }\end{array}$ & [228] \\
\hline & $\begin{array}{c}\text { 6-OST-1, 6-OST-2 and } \\
6-O S T-3\end{array}$ & $\begin{array}{l}\text { Mouse liver } \\
\text { cDNA }\end{array}$ & $\begin{array}{c}\text { Human Embryonic } \\
\text { Kidney } 293 \text { Cells (HEK } \\
293 \text { cells) }\end{array}$ & [229] \\
\hline & $d H S 6 S T$ & $\begin{array}{c}\text { Drosophila } \\
\text { melanogaster } \\
\text { cDNA }\end{array}$ & COS-7 cells & [230] \\
\hline \multirow[t]{3}{*}{$\begin{array}{l}\text { Heparan sulphate } \\
N \text { - } \\
\text { sulphotransferase } \\
\text { (NST) }\end{array}$} & $\begin{array}{l}\text { A truncated version of } \\
\text { Heparan sulfate/heparin } \\
\text { N-deacetylase/N- } \\
\text { sulfotransferase-1 } \\
(\text { NDST-1) }(r N D S T-1)\end{array}$ & Rat liver cDNA & S. cerevisiae & [231] \\
\hline & NDST-1 & Mouse cDNA & \multirow[t]{2}{*}{ HEK 293 cells } & $\begin{array}{l}{[232,} \\
233]\end{array}$ \\
\hline & NDST-2 & Murine cDNA & & [234] \\
\hline \multirow[t]{3}{*}{$\begin{array}{c}\text { Phenol } \\
\text { sulphotransferase }\end{array}$} & $\begin{array}{l}\text { Hydroxyarylamine (or } \\
\text { acetylaminofluorene) } \\
\text { sulfotransferases } \\
\text { (SULT1C) }\end{array}$ & Human cDNA & E. coli BL21 (DE3) & [235] \\
\hline & $\begin{array}{l}\text { (rabSULT1C2) } \\
\text { cDNA }\end{array}$ & Rabbit stomach & $\begin{array}{l}\text { E. coli } \mathrm{HB} 101 \text { and COS-7 } \\
\text { cells }\end{array}$ & [236] \\
\hline & $\begin{array}{l}\text { Different human } \\
\text { SULTs (SULT1A3, } \\
\text { SULT1C4, and } \\
\text { SULT1E1) }\end{array}$ & Human & E. coli BL21 (DE3) & [237] \\
\hline \multirow{3}{*}{$\begin{array}{c}\text { Dopa/Tyrosine- } \\
\text { Ester } \\
\text { Sulphotransferase }\end{array}$} & PST-1 cDNA & Rat liver & E. coli BL21 (DE3) & [238] \\
\hline & cDNA & Rat liver & COS-7 Cells & [239] \\
\hline & SULT1A3 cDNA & Human & $\begin{array}{l}\text { S. cerevisiae and E. coli } \\
\text { BL21(DE3) }\end{array}$ & [240] \\
\hline
\end{tabular}


This part of the work focused on heterologous expression of some algal fucosyltransferases (FucTs) and sulphotransferases (STs) from genes isolated from E. siliculosus in E. coli BL21 (DE3) as a trial to produce active and right-folded over-expressed enzymes.

\subsection{Heterologous expression of algal fucosyltransferases (FucTs)}

FucTs are a subfamily of the super family glycosyltransferases (GTs) according to CarbohydrateActive enZymes (CAZy) [241]. GTs catalyze different glycosidic bonds formation using mainly an active sugar containing a nucleoside phosphate or a lipid phosphate leaving group as donor substrates [242]. They are further classified into five classes according to the glycosidic linkages they synthesize; namely $\alpha-1,2-, \alpha-1,3-, \alpha-1,4-, \alpha-1,6-$, and $O$-fucosyltransferases [243]. They function Lfucose transfer specifically from GDP-L-fucose to protein [244] and glycan forming fucosides or fucosylated glycoconjugates [245].

FucTs are involved in a number of potential and versatile functions through synthesis of compounds that are involved in different physiological and pathological processes in prokaryotic or eukaryotic organisms. They include fertilization, neuronal development, immune responses, and cell adhesion [246], such as synthesis of Lewis (Le) antigens which are glycoproteins associated with the human ABO blood group system [247]. They are expressed on red blood cells, kidney and gastrointestinal epithelium by contribution from FUT3 $(\alpha-1,3)$ and FUT2 $(\alpha-1,2)$. Even in terrestrial plants, it was observed an increment in Fuc-T $(\alpha-1,4)$ in plant life cycle during flower development resulting in pollen maturation and pollen tube elongation [248]. On the other side, expression of FucTs is increased, especially FUT4 $(\alpha-1,3)$, in some cancer cell lines resulting in cancer promotion and metastasis [249]. Therefore, fucosylated molecules are considered as cancer biomarkers and beneficial in cancer diagnosis and potential targets in treatment [250]. Besides, different H. pylori FucTs such as $\alpha-1,2$ and $\alpha-1,3 / 4$ FucTs participate in the biosynthesis of its carbohydrate complex Lewis antigen which plays an important role in the bacterial pathogenesis including cell adaptation, adhesion and colonization [221,251].

Few bacterial FucTs (e.g., $\alpha-1,3 / 4-F u c T s$ from $H$. pylori) and human (H. sapiens) analogues were well identified and specified by expression in different cell lines [211,246], as previously demonstrated in Table 14. Since oceans and seas represent $70 \%$ of our planet surface, enzyme analogues from aquatic organisms could be also possible, if their genomic maps are well analyzed. 
Recently, genome analysis of E. siliculosus revealed the presence of different kinds of FucTs including $\alpha$-1,3-FucTs (GT10) such as Esi_0050_0098, $\alpha$-1,6-FucTs (GT23) (e.g., Esi0135_0016 and Esi0540_0004) as well as $O$-FucTs (GT65) like Esi0021_0026 [192]. Theses diversity in algal FucTs proved that marine life has still treasures which have not been discovered yet.

Heterologous expression is a well-documented tool in verifying bioinformatics results and gene functions, this part aimed to over-express representatives from algal FucTs; FucTs_21 and FucTs_50 in E. coli BL21 (DE3) via cloning of Esi0021_0026 and Esi0050_0098 genes, respectively. 


\subsubsection{Material and Methods}

4.2.1.1. In silico analysis of FucTs analogues, synthetic gene materials and bacterial transformation

Amino acids sequences of human and bacterial FucTs analogues were aligned using DNASTAR ${ }^{\circledR}$ (Lasergene v7, MegAlign) software. Clustal W algorithm was applied to investigate the different homology studies, including percent identity and motifs in aligned sequences. H. pylori $\alpha-1,3$-FucTs (HP0651 and HP0379) and human FUT (P51993) were aligned with algal putative FucTs_50 and FucTs_21.

Synthetic Esi0050_0098 and Esi0021_0026 genes were supplied by Invitrogen GeneArt ${ }^{\circledR}$ as described by Michel, et al. [252,253] as lyophilized samples. They were inserted into pMK and pMA-T plasmid vectors, as shown in Fig. 48. E. coli JM83 competent cells hosted the foreign DNA inserts through a heat shock protocol to produce gene copies. The bacterial growth was performed at $37^{\circ} \mathrm{C}$ in $\mathrm{LB}$ medium containing their corresponding selection markers; ampicillin sodium and kanamycin sulphate. DNA constructs were afterwards extracted by NucleoSpin ${ }^{\circledR}$ Plasmid EasyPure kits (Macherey-Nagel GmbH \& Co. KG, Düren, Germany) and concentrations were measured by Nanodrop Spectrophotometer
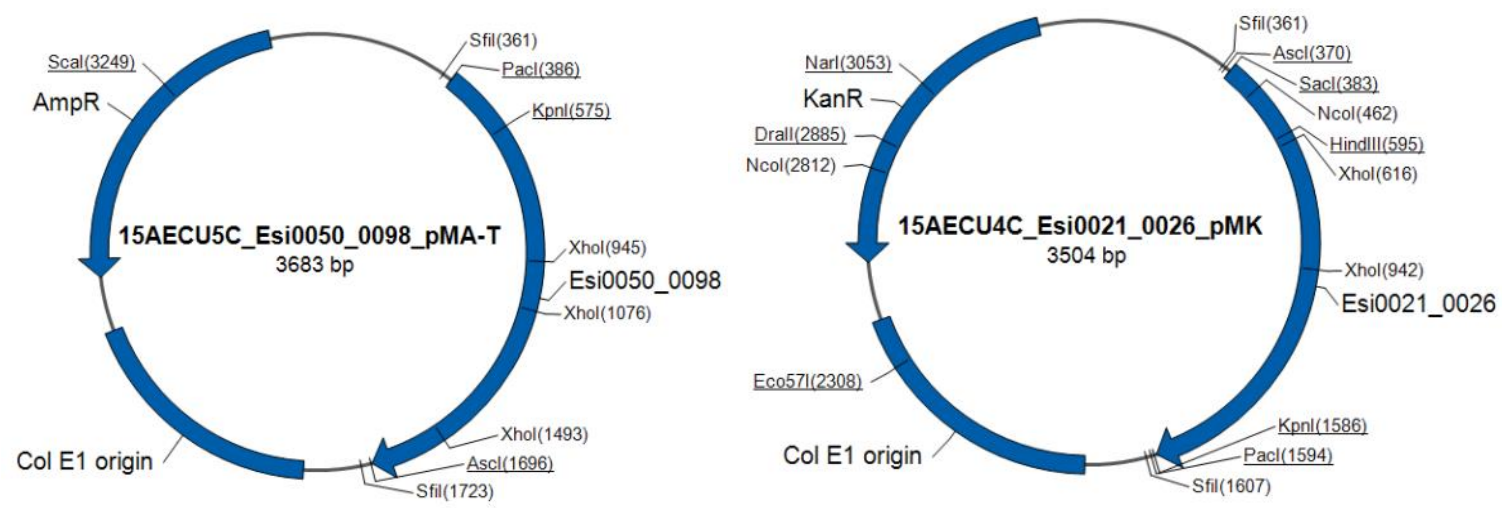

Fig. 48: Synthetic and cloned Esi0050_0098 and Esi0021_0026 in pMA-T and pMK plasmid vectors, respectively

Both DNA constructs were synthesized and provided by Invitrogen GeneArt ${ }^{\circledR}$.

\subsubsection{Cloning by Gibson assembly}

As shown in Fig. 49, between EcoRI and BamHI sites of the vector plasmid pASK-IBA 45(+) (IBA GmbH, Germany), Esi0050_0098 and Esi0021_0026 were cloned to produce Strep ${ }^{\circledR}$-tag fusion proteins. Steps were performed according to NEBuilder HiFi DNA ${ }^{\circledR}$ Assembly Master Mix and 
Gibson Assembly ${ }^{\circledR}$ Cloning Kit instruction manuals [254,255]. Primers were designed by the online service provided by New England Biolabs ${ }^{\circledR}$ (NEBuilder ${ }^{\circledR}$ Assembly Tool), as demonstrated in Table 15, and PCR experiments were performed in a PCR thermocycler to amplify both inserts.

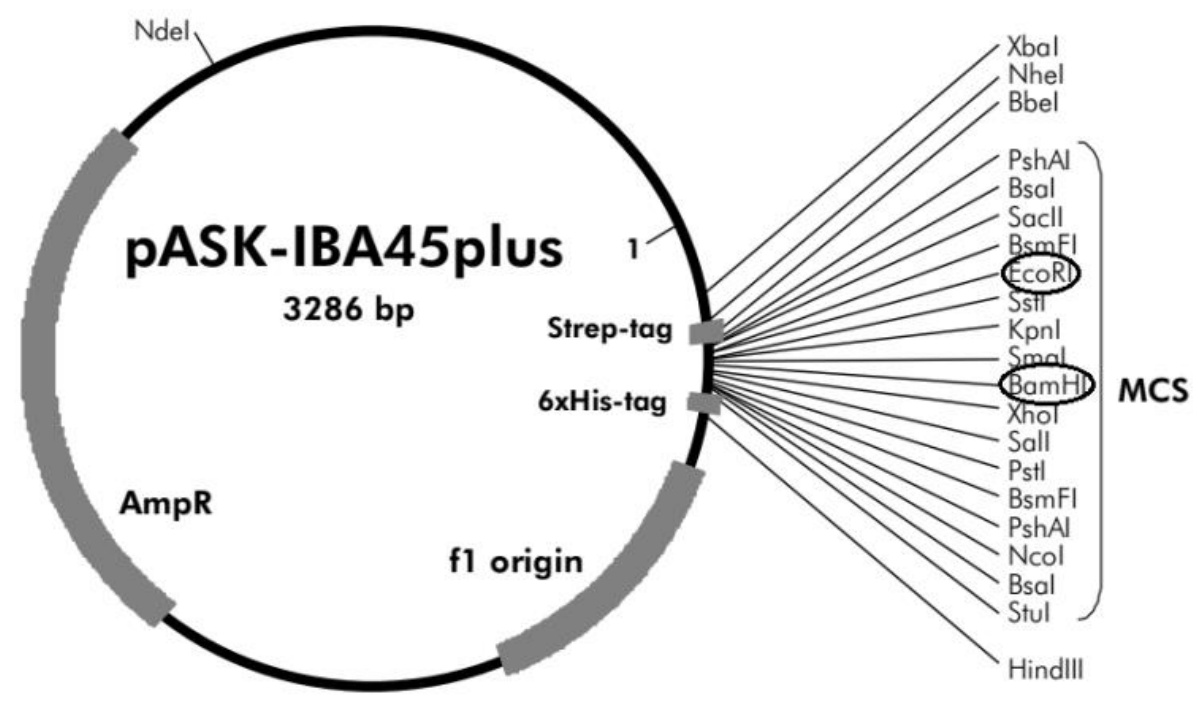

Fig. 49: Features of pASK-IBA 45(+) plasmid vector as described by IBA GmbH [256] Esi0050_0098 and Esi0021_0026 were cloned between EcoRI and BamHI site of the plasmid multiple cloning site (MCS) using Gibson assembly.

Table 15: Designed primers for cloning of Esi0050_0098 and Esi0021_0026 in pASK-IBA 45(+), according to NEBuilder ${ }^{\circledR}$ Assembly Tool

The capital and small letters denoted for nucleotides from inserts and vector, respectively.

\begin{tabular}{|c|c|c|c|}
\hline DNA constructs & \multicolumn{2}{|r|}{ Primer } & \\
\hline \multirow{2}{*}{$\begin{array}{c}\text { pASk-IBA } \\
\text { 45(+)_Esi0050_0098 }\end{array}$} & fwd & 5'-gcgccgagaccgcggtcccgATGGACAAGGAGGGCAGC-3` & \multirow[t]{2}{*}{61.3} \\
\hline & rev & 5`-cetgcaggtcgacctcgaggTCACCGCAGTGACTCTTG-3` & \\
\hline \multirow{2}{*}{$\begin{array}{c}\text { pASk-IBA } \\
\text { 45(+)_Esi0021_0026 }\end{array}$} & fwd & 5’-gcgccgagaccgcggtcccgATGGCCTTCGAGACCGTGGTCGTG-3` & \multirow[t]{2}{*}{72.0} \\
\hline & rev & 5'-cctgcaggtcgacctcgaggTCACCCCCGGGGGCGGGG-3 & \\
\hline
\end{tabular}

The molecular sizes of amplified inserts with overlapping ends were then confirmed by gel electrophoresis, detected using Gel iX20 Imager and then purified by NucleoSpin ${ }^{\circledR}$ Gel and PCR Clean-up kits (MACHEREY-NAGEL GmbH \& Co. KG).

In parallel, pASK-IBA 45(+) was opened by EcoRI and BamHI endonucleases. The purified doublydigested plasmid in addition to PCR products were incubated in a ratio of 1:2 with $10 \mu \mathrm{L}$ NEBuilder ${ }^{\circledR}$ 
HiFi DNA Assembly Master Mix in a total volume of $20 \mu \mathrm{L}$ for $15 \mathrm{~min}$ at $50{ }^{\circ} \mathrm{C}$ following the protocol of the provider (New England BioLabs ${ }^{\circledR}$ Inc.) [254]. Chemically competent cells of the supplier hosted then the recombinant DNA constructs before growing on LB solid followed by liquid media supplemented with $100 \mu \mathrm{g} \mathrm{mL} L^{-1}$ ampicillin sodium as a selection marker for $18 \mathrm{~h}$ at $37^{\circ} \mathrm{C}$.

4.2.1.3. DNA sequencing of recombinant DNA constructs

To check accuracy of molecular cloning experiments, recombinant DNA constructs were afterwards extracted by NucleoSpin ${ }^{\circledR}$ Plasmid EasyPure kits (Macherey-Nagel GmbH \& Co. KG, Düren, Germany). Isolated pASK-IBA 45(+)_Esi0021_0026 (4458 bp) was doubly digested by StuI and XbaI, while pASK-IBA 45(+)_Esi0050_0098 (4566 bp) was only linearized by a single digestion with XbaI. All of digested constructs were checked by gel electrophoresis.

Right constructs were sent for sequencing and compared their results with the designed templates by GATC Biotech AG (Konstanz, Germany), after preparing them for a light run sequencing in a $1.5 \mathrm{~mL}$ reaction tube which consisted of $100 \mathrm{ng}$ DNA, $5 \mu \mathrm{L}$ fwd/rev plasmid primer (20 pmol) and sterile water up to $10 \mu \mathrm{L}$. Alignment analysis was performed then with the online service of Clustal Omega (EMBL-EBI).

\subsubsection{Transformation of E. coli BL21 (DE3) and gene expression}

Both sequenced DNA constructs and empty vector plasmid were applied to transform E. coli BL21 (DE3) (Agilent Technologies, Inc.). In shaking flasks using a shaker incubator (Innova ${ }^{\circledR} 44$ ), transformed E. coli were cultivated in $1 \mathrm{~L} \mathrm{LB}$ culture medium supplemented with $100 \mu \mathrm{g} \mathrm{mL}^{-1}$ ampicillin sodium at $37{ }^{\circ} \mathrm{C}$ as a pilot experiment. Proteins expression was induced by $150 \mu \mathrm{g} \mathrm{L}^{-1}$ AHT, when bacterial growth log phase was reached (i.e., OD $578 \mathrm{~nm}$ between $0.6-0.8$ ). The cultivation temperature was then decreased to $17{ }^{\circ} \mathrm{C}$ and held for overnight $(18 \mathrm{~h})$. Cells were harvested by superspeed centrifuge at $4{ }^{\circ} \mathrm{C}(10 \mathrm{~min}$ at $17600 \mathrm{x} \mathrm{g})$ and stored at $-20{ }^{\circ} \mathrm{C}$ until purification. Scalingup of FucTs_50 production was carried out in $44 \mathrm{~L}$ of cultivation medium applying the same conditions.

4.2.1.5. Proteins purification by affinity chromatography

Expressed Strep ${ }^{\circledR}$-tag fusion proteins were purified according to the protocol described by IBA Lifesciences (Göttingen, Germany) [257] after some modifications. Cells were reconstituted in buffer W without EDTA (100 mM Tris, $150 \mathrm{mM} \mathrm{NaCl,} \mathrm{pH} \mathrm{8.0).} \mathrm{Afterwards,} \mathrm{cells} \mathrm{were} \mathrm{lysed} \mathrm{with}$ a microfluidizer adjusted at 15000 psi after incubation with of DNaseI and lysozyme for 30 min. The step of cell disruption was repeated for three times. To isolate cell debris from cell lysates, cell 
suspensions were centrifuged again at $4^{\circ} \mathrm{C}$ for $1 \mathrm{~h}$ at $43000 \mathrm{x} g$. Clear lysates were then filtered by a $0.45 \mu \mathrm{m}$ regenerated cellulose syringe filter and purified with a Strep-Tactin ${ }^{\circledR}$ Sepharose ${ }^{\circledR}$ affinity column. The column was pre-conditioned with buffer $\mathrm{W}$ and bound proteins were eluted with five column volume $(\mathrm{CV})$ of buffer $\mathrm{E}(2.5 \mathrm{mM}$ desthiobiotin in buffer $\mathrm{W})$ before a step of washing with buffer W.

\subsubsection{Protein detection by SDS-PAGE and Western blot}

Column fractions were plotted on SDS-PAGE (12.5\%) using Vertical electrophoresis cell for SDSPAGE and proteins were stained by Coomassie brilliant blue solution. Moreover, recombinant Strep ${ }^{\circledR}$-tag fusion proteins were detected by Western blot using monoclonal Strep ${ }^{\circledR}$-Tactin ${ }^{\circledR}$ AP conjugate (IBA Lifesciences, Göttingen, Germany) according to manufacturer's instructions[258].

\subsubsection{Activity assays}

a. Glycosyltransferase activity kit

Activity assays were performed according to the protocol of Glycosyltransferase activity kit (Catalog \# EA001) described by Bio-Techne GmbH [259]. Briefly, purified enzymes were dialyzed against the reaction buffer (25 mM Tris, $150 \mathrm{mM} \mathrm{NaCl}, 10 \mathrm{mM} \mathrm{MnCl}_{2}, 10 \mathrm{mM} \mathrm{CaCl}_{2}, \mathrm{pH}$ 7.5) for overnight and concentrated using Vivaspin centrifugal concentrators (MWCO $10 \mathrm{kDa}, \mathrm{VWR}$ ). The reaction was initiated by incubation of $25 \mu \mathrm{L}$ a serial dilution of purified protein in a working solution consisting of $10 \mu \mathrm{L} 8 \mathrm{mM}$ GlucNAc, $10 \mu \mathrm{L} 3 \mathrm{mM}$ GDP-L-fucose (Carbosynth Limited, Berkshire, United Kingdom), $5 \mu \mathrm{L} 20 \mathrm{ng} \mu \mathrm{L}^{-1}$ coupling phosphatase for $1 \mathrm{~h}$ at $37{ }^{\circ} \mathrm{C}$. Afterwards, $30 \mu \mathrm{L}$ malachite green A (ammonium molybdate in $3 \mathrm{M}$ sulphuric acid), $100 \mu \mathrm{L}$ water and $30 \mu \mathrm{L}$ malachite green B (malachite green oxalate and polyvinyl alcohol) were added before another incubation at room temperature for $20 \mathrm{~min}$. Finally the absorbance was measured at $620 \mathrm{~nm}$ with reaction buffer as a blank to measure the reaction background.

b. Multiplexed Capillary Electrophoresis (MP-CE)

MP-CE was carried out as described previously by Wahl, et al. [260]. Briefly, samples were diluted with a stock solution, containing $14 \mathrm{mM}$ SDS, $2 \mathrm{mM}$ para-amino benzoic acid (PABA) and $2 \mathrm{mM}$ para-amino phtalic acid (PAPA), to a final concentration of $7 \mathrm{mM} \mathrm{SDS,} 1 \mathrm{mM}$ PABA and $1 \mathrm{mM}$ PAPA. SDS was used to denature the enzyme, while PABA and PAPA were internal standards. Precipitated enzyme was removed by centrifugation and the supernatants were applied with a vacuum injection at -0.7 psi for $10 \mathrm{~s}$. Separation was carried out with a capillary electrophoresis (cePRO $9600^{\mathrm{TM}}$ ) system with a 96 fused silica capillary array and a UV-detection at $254 \mathrm{~nm}$. 
Capillary tubes with a total length of $80 \mathrm{~cm}$, i.d. of $50 \mu \mathrm{m}$ and $55 \mathrm{~cm}$ as an effective length were used. Analytes were separated with an applied voltage of $12 \mathrm{kV}$ and the used running buffer contained $1 \mathrm{mM}$ EDTA and $50 \mathrm{mM}$ ammonium acetate at $\mathrm{pH} 9.2$. Integrated analytical areas were normalized to the internal standard peak area of PABA. 


\subsubsection{Results and Discussion}

According to Cumashi, et. al., $\alpha-1,3$ linked L-fucopyranoside is principally found in fucoidan backbone among different studied molecular structures from brown macroalgae species. However, other linkages such as $\alpha-1,4$ linkage might also alternate with $\alpha-1,3$ [14]. Moreover, branched chain fucoidan contains further $\alpha-1,2$ or even $\alpha-1,6$ residues as branching sites. Genome analysis of $E$. siliculosus proved the presence of different genes encoded for different FucTs involved in different glyosidic linkages like $\alpha-1,3$ and $\alpha-1,6$ [192].

4.2.2.1. Homology and phylogenetic relationships with relative FucTs

Results from NCBI database showed that amino acid sequences of both algal FucTs are novel and different from other known glycosyltransferases [240,241]. As demonstrated in Fig. 50, homology studies of amino acids sequences of both algal FucTs; FucTs_50 (Esi0050_0098 (D7G396)) and FucTs_21 (Esi0021_0026 (D7FR32)) with the well specified H. pylori $\alpha-1,3 / 4-F u c T s ~(H P 0651$ and HP0379) [211,263] in addition to the human analogue FUT6 (P51993), revealed that FucTs_50 was more related to bacterial $\alpha-1,3 / 4$-analogues than FucTs_21, where identity (\%) to HP0651 and HP0379 was $14.7 \%$ and $15.4 \%$, respectively, however, it was only 9.8\% and 9.7\% with FucTs_21, respectively. This result was likely due to the fact that FucTs_21 is a $O$-FucTs rather than and a $\alpha$ 1,3-FucTs. Furthermore, the comparison between algal FucTs_50, H. pylori and human $\alpha$-1,3-FucTs demonstrated more relations of algal enzymes to the human analogue than the bacterial one. 


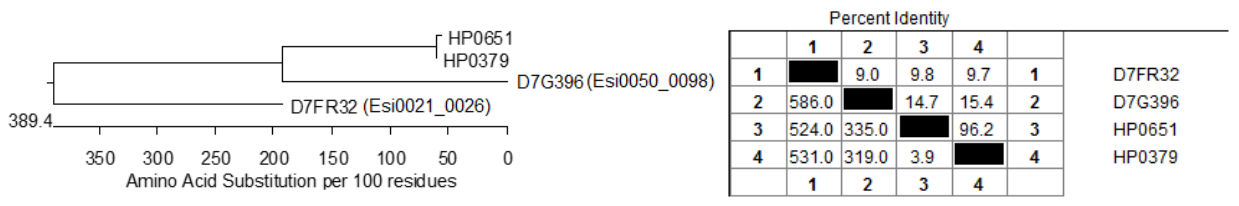

a

b

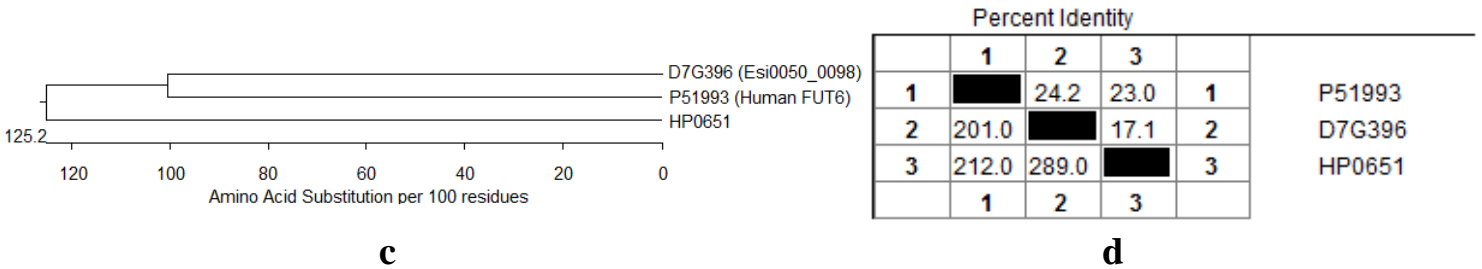

Fig. 50: Homology and phylogenetic relationships of algal FucTs_21 and FucTs_50 with other bacterial and human FucTs

(a) Phylogenetic tree shows more similarity of FucTs_50 (Esi0050_0098, D7G396) with H. pylori enzymes than FucTs_21 (Esi0021_0026, D7FR32). (b) Sequence distance matrix confirmed phylogenetic tree regarding identity $(\%)$. (c\&d) Phylogenetic tree and sequence distance matrix of algal FucTs_50 (Esi0050_0098, D7G369) in comparison with bacterial H. pylori (HP0651) and human (P51993) analogues. DNASTAR ${ }^{\circledR}$ (Lasergene v7, MegAlign) software and Clustal W algorithm were applied to investigate the different homology studies, including percent identity, phylogenetic tree and motifs in aligned sequences

These lower percentages of identity might indicate different enzymatic functions. However, different enzymes' resources should be taken into consideration. Furthermore, amino acid sequence of FucTs_50 revealed the presence of the conserved stretch of 17 amino acids; FLLAFENNNQIRDYVTEK. This consensus sequence is referred as a $\alpha 3$-FucTs structural motif after alignment of several $\alpha 3$-FucTs from bacteria and human [264]. It is believed that this sequence is essential for enzyme activity and related to GDP-L-fucose binding site. In addition, alignment of FucTs_21 amino acid sequence with other protein- $O$-fucosyltransferases family 1 (POFUT1), revealed the presence of some conserved peptide motifs for example LxYIATD and SSFxA). These POFUT1 include enzymes present in H. sapines, S. scrofa, G. gallus ...etc. genome [265].

\subsubsection{Molecular cloning by Gibson assembly}

Gibson assembly performed, as shown in Fig. 51, by three successive enzymatic activities in a single-tube reaction: 5' exonuclease, DNA polymerase and DNA ligase activities. The 5' exonuclease chews back the 5' end sequences resulting in exposed complementary sequence to be annealed. The polymerase activity then fills in the gaps on the annealed regions. A DNA ligase afterwards seals 
the nick and connects the DNA fragments together. An advantage of Gibson assembly is that the overlapping sequence of the connecting fragments is longer than those used in previous assemblies (e.g., Golden Gate Assembly), which leads to a higher percentage of correct assemblies [266].

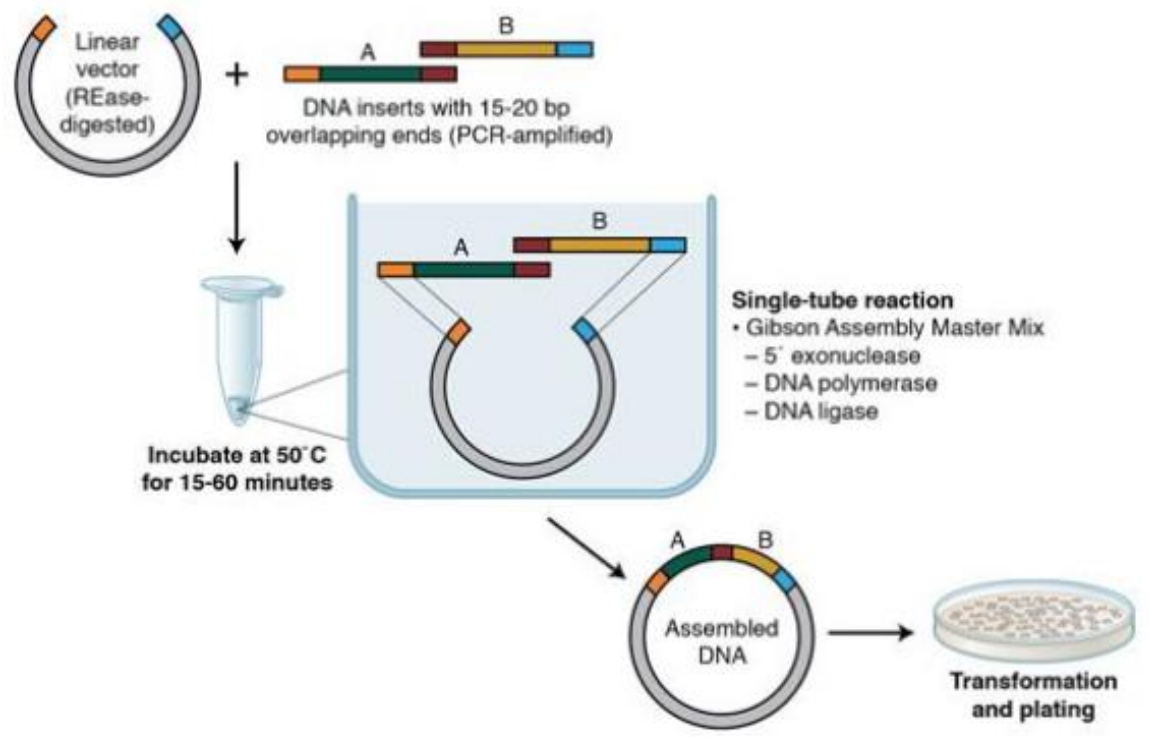

Fig. 51: Gibson Assembly work flow; an example [266]

As shown in Fig. 52, both inserts; Esi0021_0026 (1197 bp) and Esi0050_0098 (1305 bp) were amplified successfully with designed PCR primers. The ends of both amplicons included EcoRI and BamHI restriction site sequences from pASK-IBA $45(+)$ to facilitate ligation to the vector in subsequent steps. 


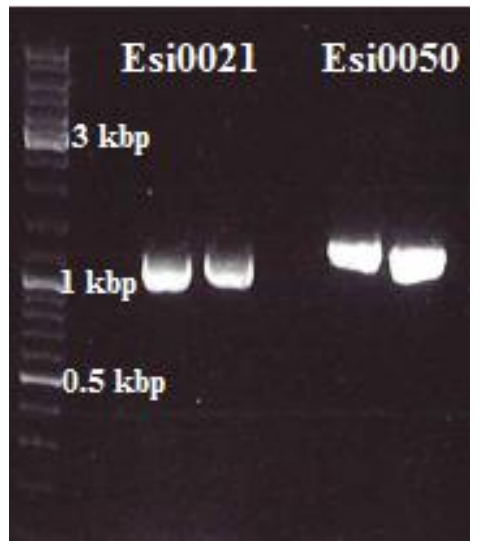

Fig. 52: Agarose gel electrophoresis of amplified PCR products of Esi0021_0026 and Esi0050_0098 Esi0021_0026 and Esi0050_0098 amplicons were detected at 1197 bp and 1305 bp, respectively with their overlapping ends.

4.2.2.3. DNA extraction, digestion and sequencing

Extracted DNA constructs were singly or doubly digested to confirm their right constructions. Fig. 53 and 54 describe the comparison between designed constructs by pDRAW32 DNA analysis software and obtained results from gel electrophoresis. In pASK-IBA 45(+)_Esi0021_0026, only recombinant DNA from replicate 3 showed two fragments at 3129 and $1329 \mathrm{bp}$, upon digestion with StuI and XbaI, as predicted. However, in pASK-IBA 45(+)_Esi0050_0098, recombinant DNA from replicate 3 and 8 showed a typical gel electrophoresis profile with a single band at 4566 bp after a digestion with $\mathrm{XbaI}$. 


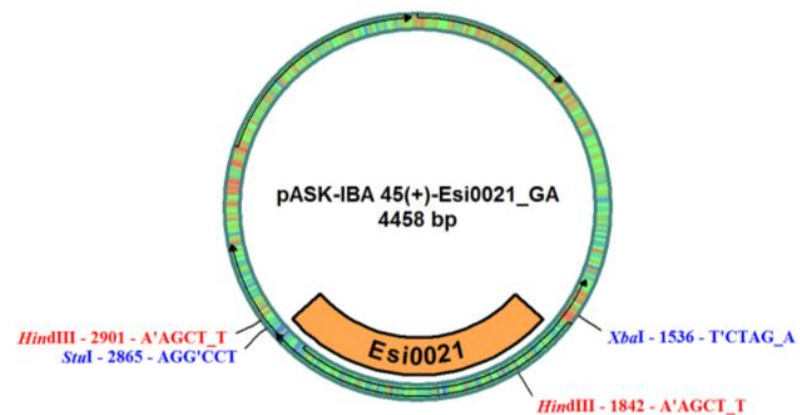

$\mathbf{a}$

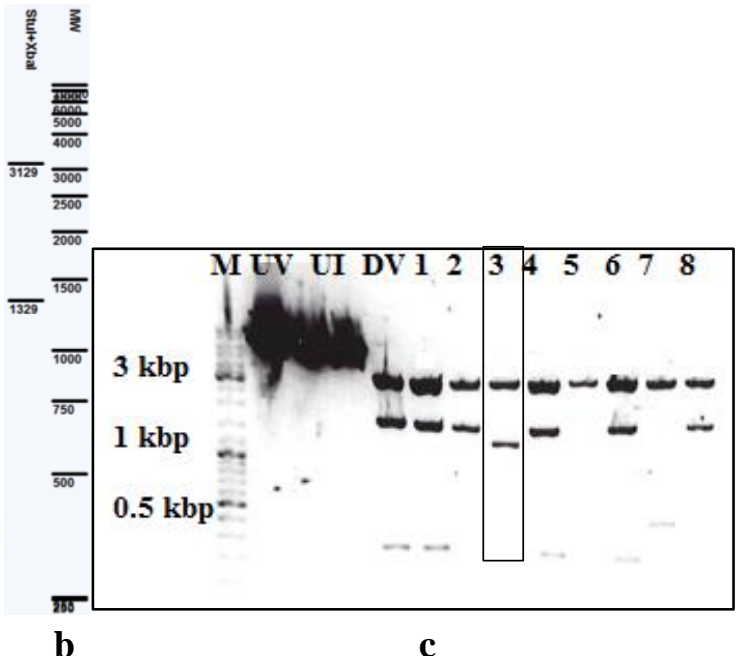

b

c

Fig. 53: Design of pASK-IBA 45(+)_Esi0021_0026 DNA construct showing some possible restriction sites and gel electrophoresis results after its digestion with $S t u I$ and $X b a I$

a) Designed construct by pDRAW32 DNA analysis software; b) Expected produced fragments after double digestion with $S t u \mathrm{I}$ and $X b a \mathrm{I}$ at 3129 and $1329 \mathrm{bp}$, c) Gel electrophoresis of recombinant DNA

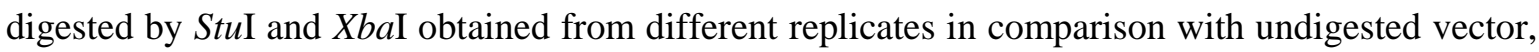
undigested insert, digested vector and DNA marker. Only replicate 3 produced two fragments at 3129 and 1329 bp. $\mathrm{M}=$ size marker, $\mathrm{UV}=$ undigested vector, $\mathrm{UI}=$ undigested insert, $\mathrm{DV}=$ digested vector.

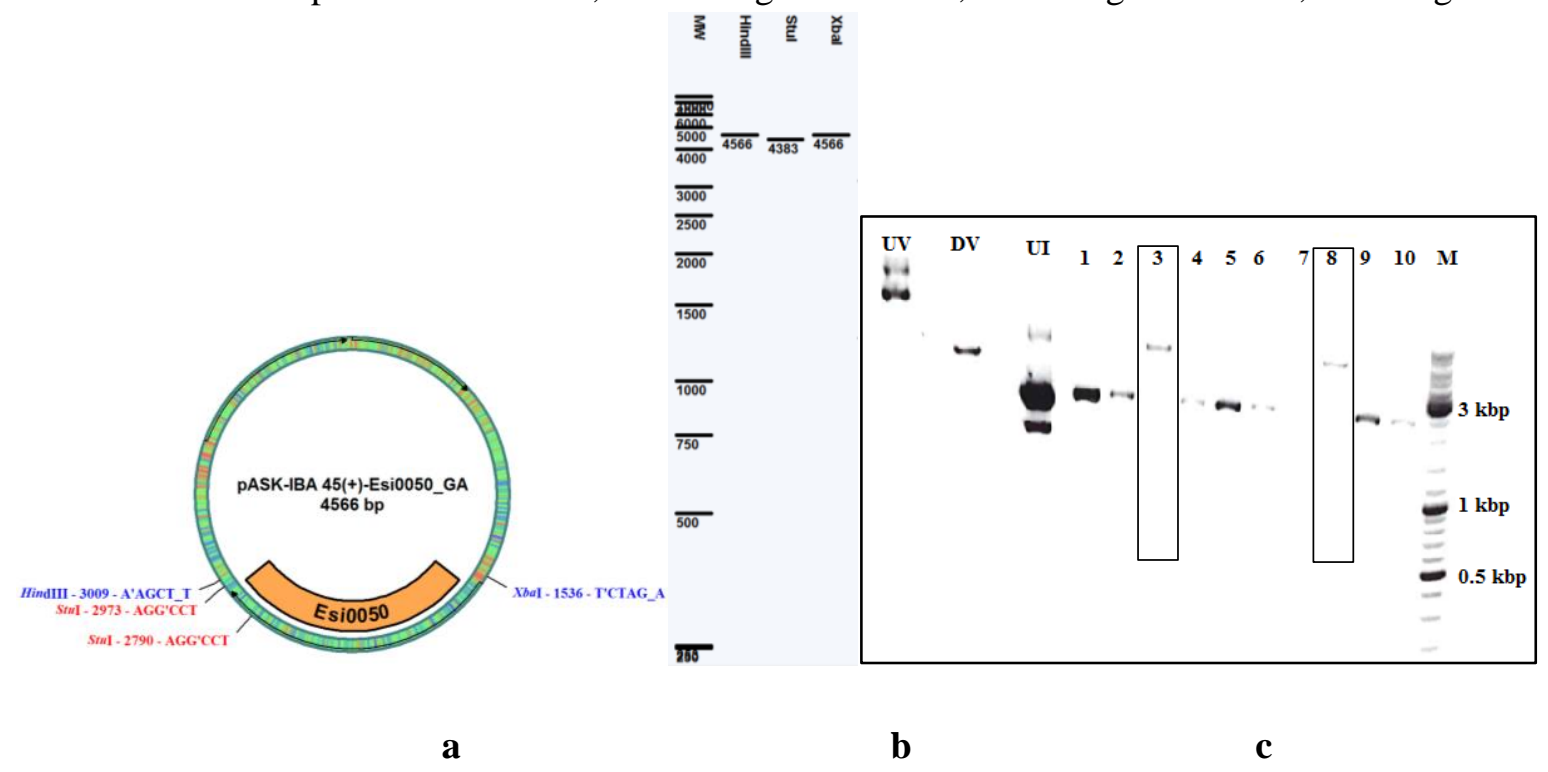

Fig. 54: Design of pASK-IBA 45(+)_Esi0050_0098 DNA construct showing some possible restriction enzymes and gel electrophoresis results after digestion $\mathrm{XbaI}$

a) Designed pASK-IBA 45(+)_Esi0050_0098 construct pDRAW32 DNA analysis software; b) Expected fragments after single digestion with $X b a \mathrm{I}$ at $4566 \mathrm{bp}$; c) Gel electrophoresis of recombinant DNA digested by $X b a \mathrm{I}$ obtained from different prep in comparison with undigested vector, undigested insert, digested vector and a DNA size marker. Digested DNA from replicates 3 and 8 showed slower migration rates than digested vector, this refered to higher molecular weight of 


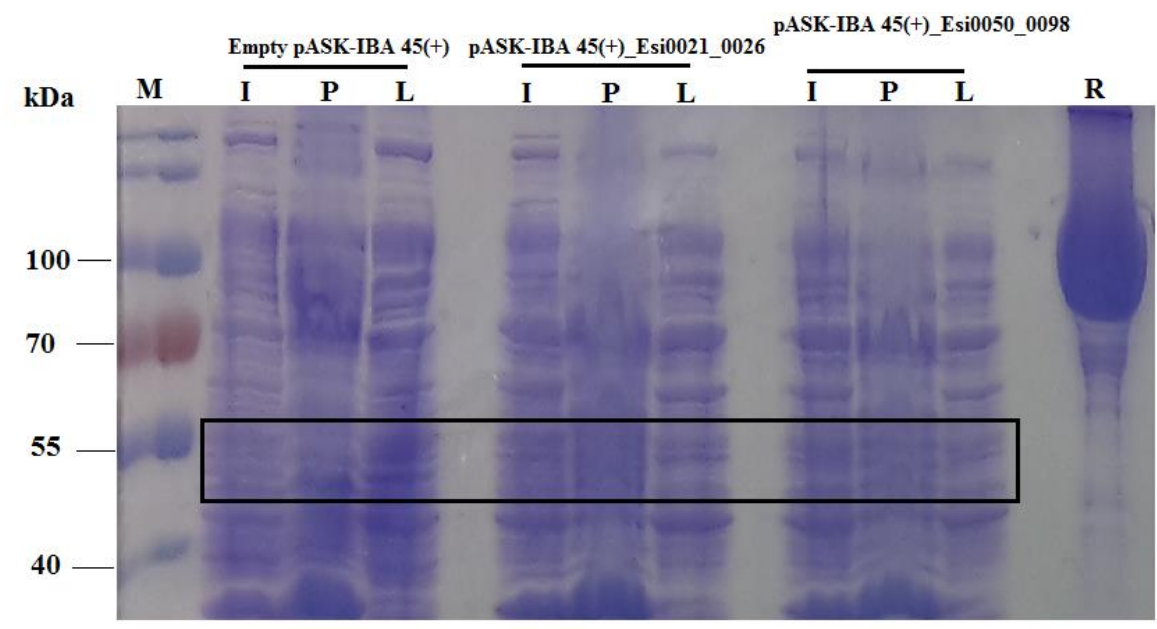

a

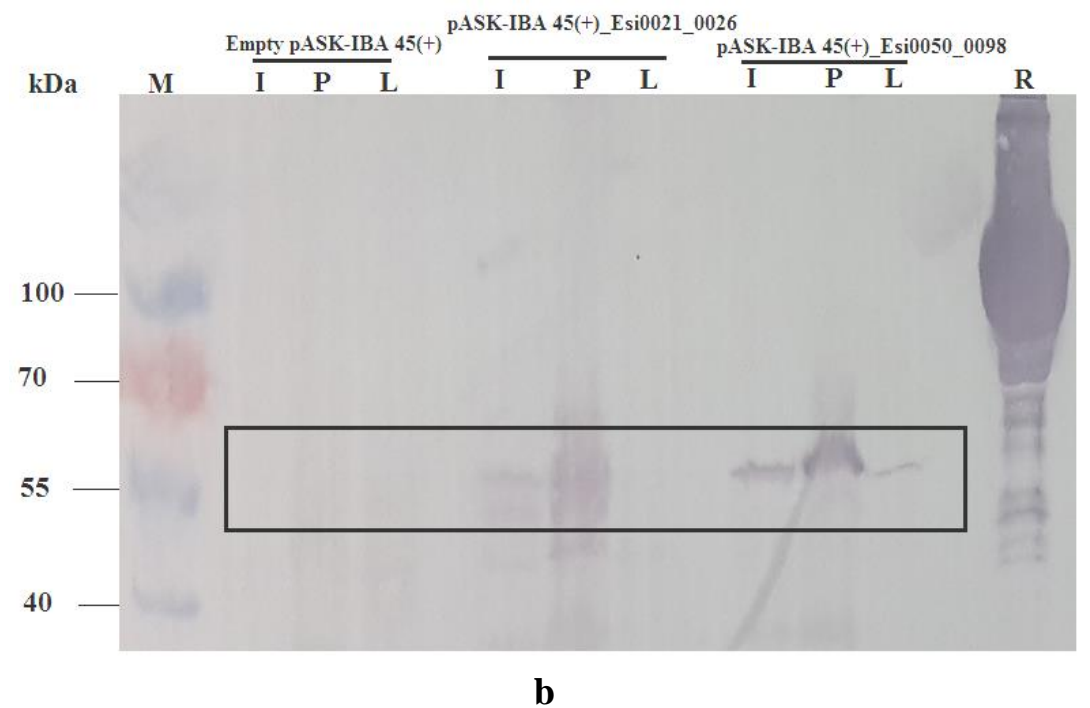

Fig. 56: SDS-PAGE (a) and Western blot (b) of overexpressed recombinant proteins from pASK_IBA 45(+)_Esi0021_0026 and pASK_IBA 45(+)_Esi0050_0098 DNA constructs, in comparison with an empty vector plasmid in a $2 \mathrm{~L}$ of $\mathrm{LB}$ medium of $E$. coli BL21(DE3)

Proteins were detected in Western blot by Strep $^{\circledR}$ antibodies, according to IBA[258]. M= size marker - PageRuler ${ }^{\mathrm{TM}}$ Prestained (Thermo Fisher); I= intact cells; $\mathrm{P}=$ cell pellets; $\mathrm{L}=$ cell lysate; $\mathrm{R}=$ external reference Strep ${ }^{\circledR}$-tag protein of molecular size $100 \mathrm{kDa}$ used to check the the detection procedure.

\subsubsection{FucTs_50 production and purification}

Based on preliminary results, scaling-up of FucTs_50 production and purification was only performed and its activity was evaluated, afterwards. Over-expressed FucTs_50 crude lysate, produced from a $44 \mathrm{~L}$ cultivation medium, was pooled and purified by affinity chromatography depending on interaction with the immobilized engineered streptavidin named Strep-Tactin ${ }^{\circledR}$. After 
a washing step, elution of recombinant protein was performed by adding $2.5 \mathrm{mM}$ desthiobiotin in buffer W. Application of desthiobiotin depends on structural similarity to biotin which is the natural ligand of streptavidin. Finally after eluate dialysis and concentration, the experiment resulted in production of $175 \mu \mathrm{g}$ of purified FucTs_50 protein, after its measurement by Bradford assay [267].

Fig. 57 shows the cascade of FucTs_50 protein purification and its detection in column elautes in comparison with expressed proteins at the same fermentation conditions from empty plasmid vector pASK-IBA $45(+)$.

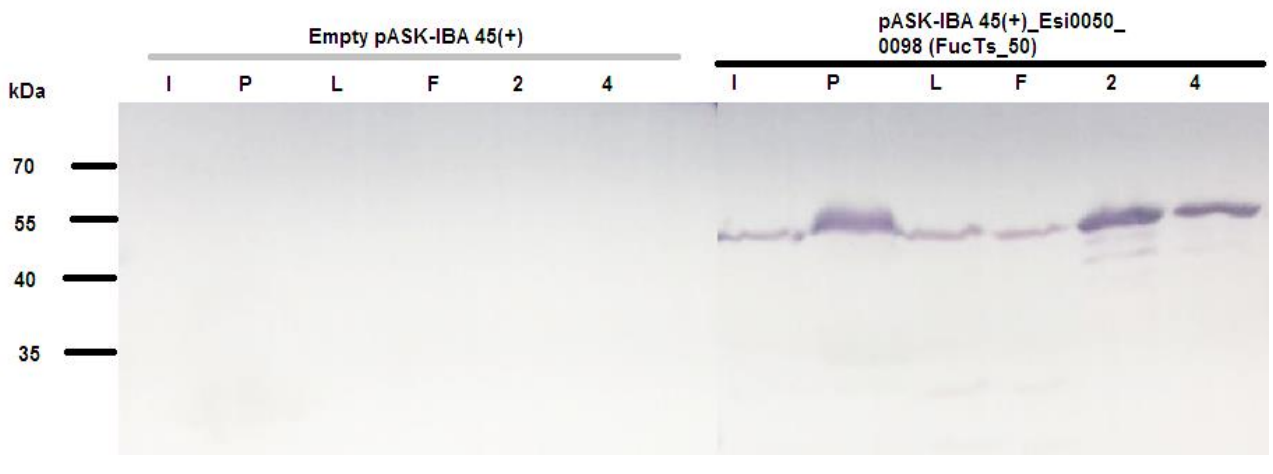

Fig. 57: Western blot of expressed recombinant FucTs_50 from the pASK-IBA 45(+)_Esi0050_0098 construct in comparison with empty vector plasmid during purification cascade

It showed that proteins were present in intact cells, cell pellets, lysate, and flow through, in addition to eluate fractions. All fractions of expressed empty vector at the same conditions did not show any presence of strep $^{\circledR}$-tag proteins. $\mathrm{R}=$ external reference Strep ${ }^{\circledR}$-tag protein of molecular size 100 $\mathrm{kDa}$; I=Intact cells; $\mathrm{P}=$ cell pellets; $\mathrm{L}=$ cell lysate; $\mathrm{F}=$ flow through; $2,4=$ eluate fractions.

\subsubsection{Activity assay}

Catalytic activity of purified FucTs_50 using GDP-L-fucose as a donor substrate form was investigated by Glycosyltransferase activity kit and MP-CE analytics.

a. Glycosyltransferase activity kit

As shown in Fig. 58, the principle of the reaction depends on a cascade of steps which initiated with hydrolysis of GDP-L-fucose with the FucTs and terminated with free phosphate detection. The step of GDP-L-fucose hydrolysis was accompanied by liberation of a free GDP moiety, which was further hydrolyzed by coupling phosphatase activity to give free phosphate and guanosine monophosphate. Malachite green reagents (malachite green A and malachite green B) reacted afterwards with these free phosphates and the products were quantified by measuring the absorbance at $620 \mathrm{~nm}$, as 
described by $\mathrm{Wu}$, et al. [259]. Denser green colour means much liberated free phosphates and consequently higher fucosyl transfer enzymatic activity.

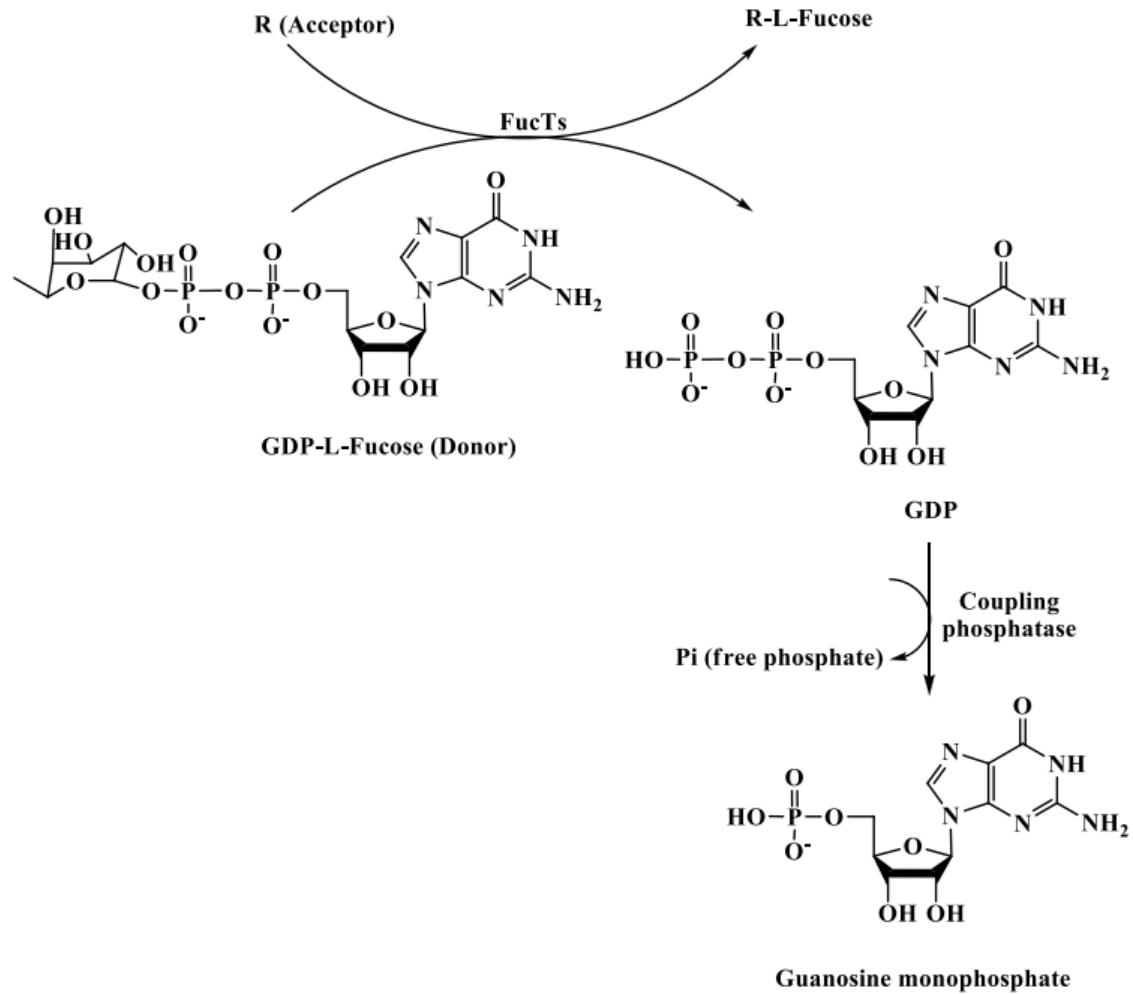

Fig. 58: Glycosyltransferase activity kit principle, as described by the supplier (Bio-Techne $\left.{ }^{\circledR}\right)$

Steps are initiated with hydrolysis of GDP-L-fucose with an active FucTs and terminated with free phosphate detection

FucTs_50 was determined in comparison with empty plasmid expressed proteins. FucTs_50 showed an activity towards GDP-L-fucose of $0.47 \mathrm{pmol} \mathrm{min}^{-1} \mu \mathrm{g}^{-1}$. It was determined according to the Eq. 7 [259], where; $\mathbf{S}$ is the slope of Fig. 59 and $\mathbf{C F}$ is the conversion factor determined from phosphate concentration calibration curve (see Appendix).

$$
\text { Specific activity }=\frac{\mathrm{S}\left(\frac{\mathrm{OD}}{\mu \mathrm{g}}\right) \times \mathrm{CF}\left(\frac{\mathrm{pmol}}{\mathrm{OD}}\right)}{\text { Time }(\min .)}
$$

Specific activity of FucTs_50 $=\frac{0.008 \times 3509.8}{60}=0.47 \mathrm{pmol} \mathrm{min}^{-1} \mu \mathrm{g}^{-1}$ 


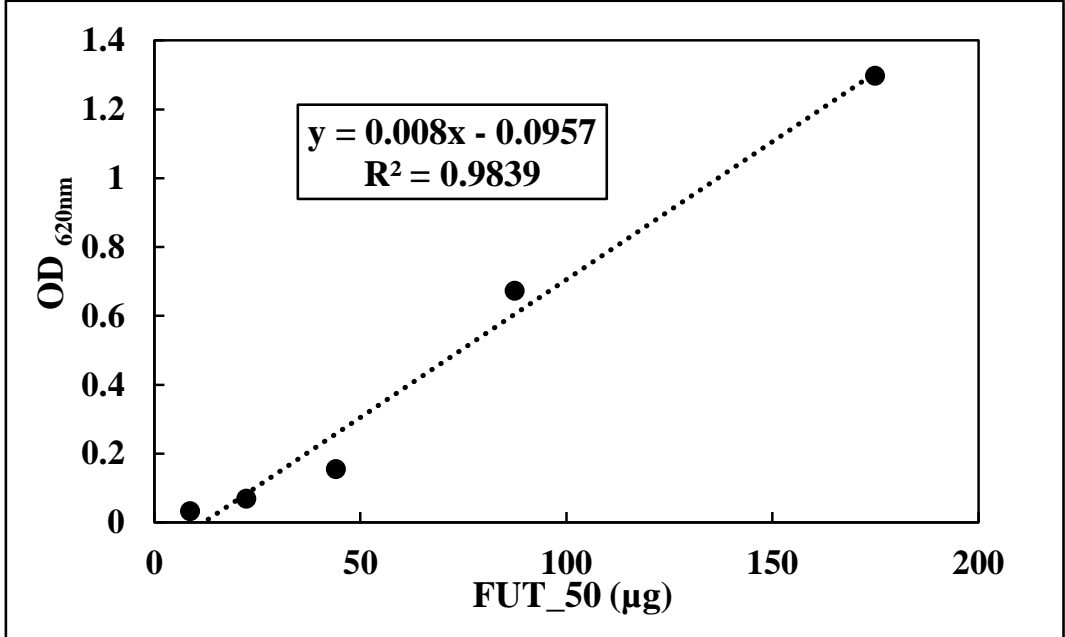

Fig. 59: Measurement of catalytic activity of FucTs_50 on the donor substrate GDP-L-fucose by Glycosyltransferase activity kit

OD values were measured at $620 \mathrm{~nm}$ to determine free liberated phosphate from GDP moiety. All absorbance values were subtracted from background value.

b. MP-CE analysis

Another hydrolytic activity was performed to investigate the putative function of FucTs_50 toward its substrate GDP-L-fucose in the absence of a suitable fucose acceptor. It was investigated by measuring GDP-L-fucose concentration after incubation with FucTs at optimum conditions. After $24 \mathrm{~h}$ incubation, as shown in Fig. 60, approx. 40\% of GDP-L-fucose was hydrolyzed in the presence of the enzyme. However, GDP-L-fucose concentration remained intact in the absence of the enzyme confirming an enzymatic catalytic activity of FucTs_50. 


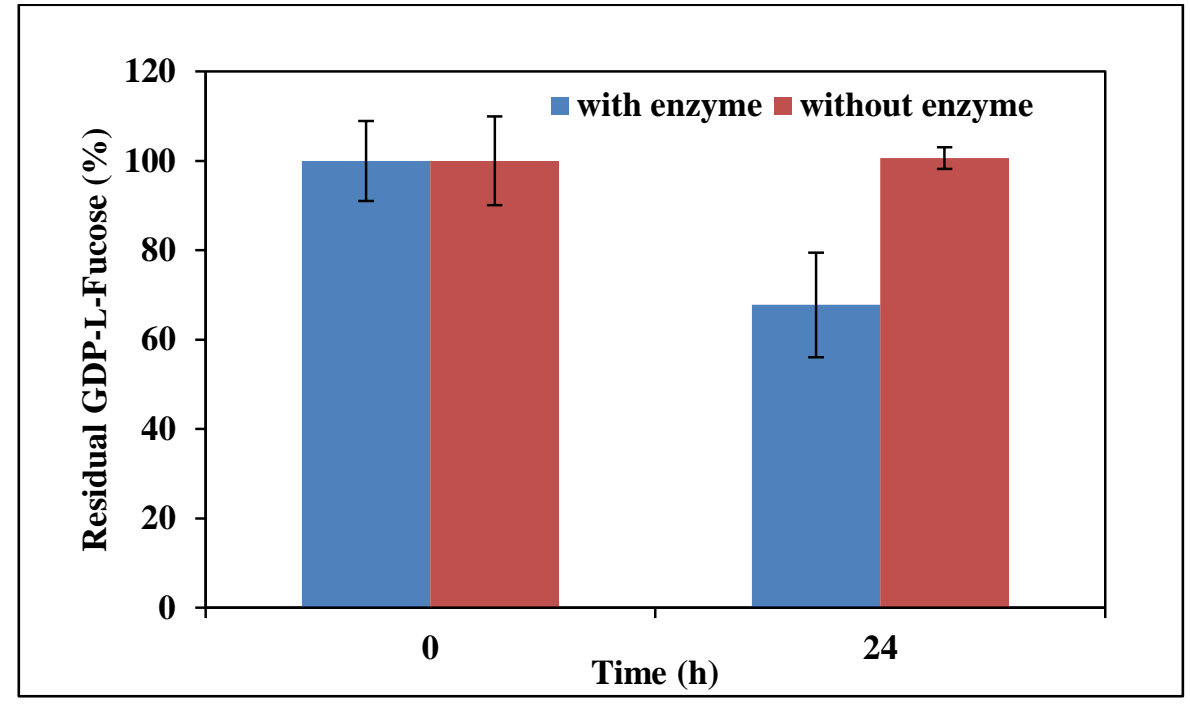

Fig. 60: FucTs_50 hydrolytic activity toward GDP-L-fucose as determined by MP-CE

Results showed that after $24 \mathrm{~h}$, FucTs_50 could cleavage approx. 40\% of GDP-L-fucose, in comparison with the enzyme-free condition. 


\subsection{Carbohydrate sulphotransferases (STs)}

Sulphotransferases (STs) catalyze the sulphonation reaction which is involved in many biological activities (e.g., detoxification and drug metabolism) [251]. They transfer sulphonate ester group $\left(\mathrm{SO}_{3}{ }^{-}\right)$from the donor activated substrate $3{ }^{`}$-phosphoadenosine 5`-phospho-sulphate (PAPS) to a hydroxyl group acceptor of specific compounds, such as phenolic and complex carbohydrates molecules in a regio- and stereo-selective reaction creating a sulphate ester $\left(\mathrm{SO}_{4}{ }^{-}\right)$. Their activity are often regulated by supply of PAPS which acts as also a cofactor, as shown in Fig. 61 [268].

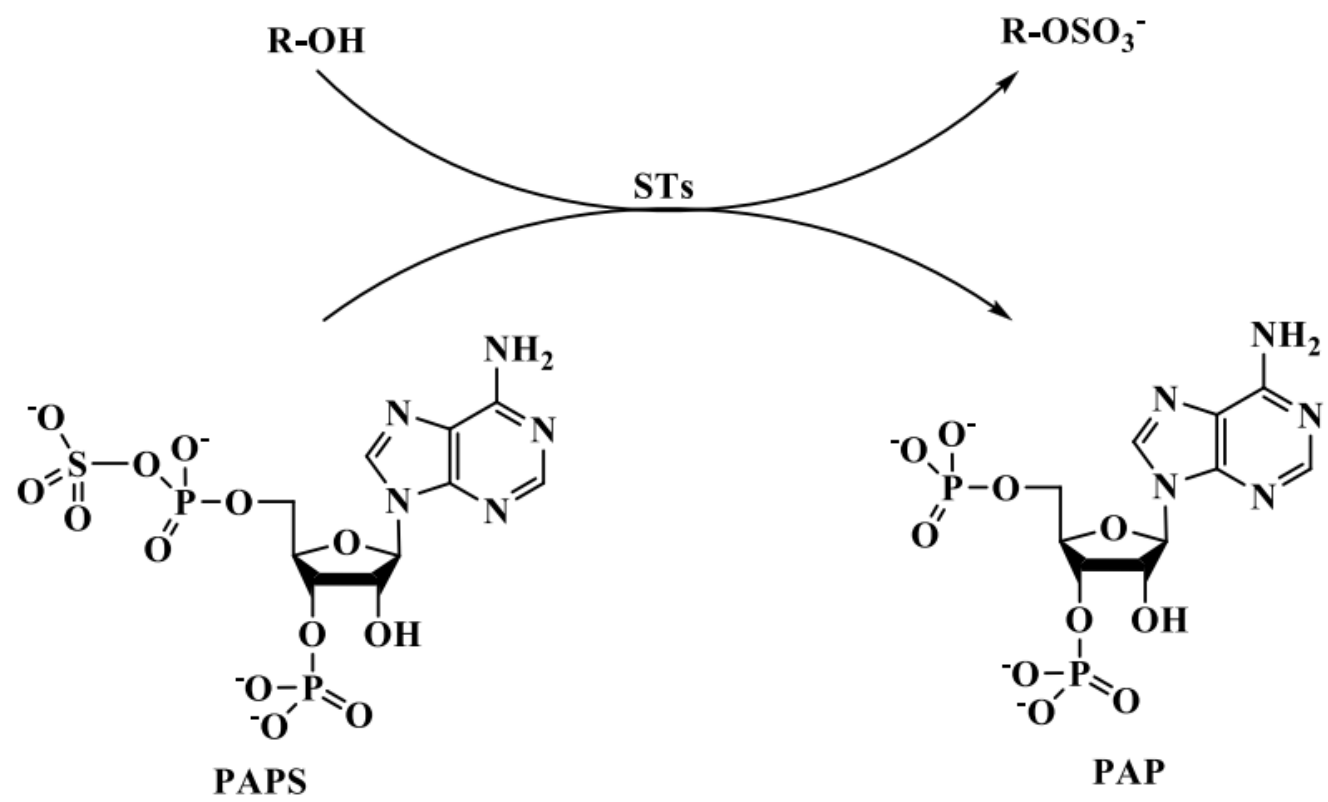

Fig. 61: Catalysis of sulphonate group $\left(\mathrm{SO}_{3}^{-}\right)$transfer to a hydroxyl group-containing compound by Sulphotransferases (STs) using PAPS as a donor substrate

E. siliculosus genome has, in addition, a gene cluster of more than 10 genes encode for different classes of carbohydrate sulphotransferases (STs). Some of these enzymes are speculated to be involved in fucoidan biosynthesis in brown macroalgae [192]. Nevertheless, the others contribute in synthesis of other unknown compounds or detoxification of water pollutants. Exact specificity and functions of most of these enzymes are still uncertain. Therefore further characterizations are required regarding to confirm their ability to sulphonate sugar monomers and contribution to fucoidan synthesis. Table 16 summarizes the different algal STs regarding their encoded genes, amino acids and putative functions [192]. 
Table 16: Overview of different algal STs, regarding their names, sizes of encoded genes, number of amino acids and putative functions

\begin{tabular}{|c|c|c|c|c|}
\hline $\begin{array}{c}\text { STs } \\
\text { name }\end{array}$ & $\begin{array}{l}\text { Encoded } \\
\text { gene }\end{array}$ & $\begin{array}{l}\text { Size of gene } \\
\text { (nucleotides) }\end{array}$ & No. of amino acids & Putative functions \\
\hline STs_28 & Esi0028_0011 & 1026 & 342 & \multirow[t]{4}{*}{ Aryl sulphotransferase } \\
\hline STs_411 & Esi0411_0021 & 837 & 279 & \\
\hline STs_442 & Esi0442_0008 & 1026 & 342 & \\
\hline STs_197 & Esi0197_0025 & 1002 & 334 & \\
\hline STs_289 & Esi0289_0025 & 1044 & 348 & $\begin{array}{l}\text { Transfer of sulphate group to } \\
\text { specific compounds (unclear } \\
\text { specificity) }\end{array}$ \\
\hline STs_210 & Esi0210_0041 & 1392 & 464 & \multirow{2}{*}{$\begin{array}{c}\text { Related to Chondroitin 6- } \\
\text { sulphotransferase and keratan } \\
\text { sulphate Gal-6 sulfotransferase } \\
\text { (unclear specificity) }\end{array}$} \\
\hline STs_312 & Esi0312_0029 & 1272 & 424 & \\
\hline STs_32 & Esi0032_0064 & 1386 & 462 & \multirow{4}{*}{$\begin{array}{c}\text { Related to Galactose-3-O- } \\
\text { sulphotransferase } 2 \text { (unclear } \\
\text { specificity) }\end{array}$} \\
\hline STs_283 & Esi0283_0018 & 1032 & 344 & \\
\hline STs_57 & Esi0057_0043 & 939 & 313 & \\
\hline STs_118 & Esi0118_0049 & 1029 & 343 & \\
\hline STs_37 & Esi0037_0054 & 1458 & 486 & \multirow{4}{*}{$\begin{array}{l}\text { Related to heparan sulfate } \\
\text { glucosamine 3-O- } \\
\text { sulphotransferase (unclear } \\
\text { specificity) }\end{array}$} \\
\hline STs_80 & Esi0080_0060 & 1044 & 348 & \\
\hline STs_26 & Esi0026_0167 & 1644 & 548 & \\
\hline STs_239 & Esi0239_0035 & 1680 & 560 & \\
\hline
\end{tabular}

Esi0032_0064 and Esi0283_0018 encode for STs_32 and STs_283 respectively are distantly related to Galactose-3- $O$-sulfotransferase 2 (EC 2.8.2.-) [269,270]. However, the other genes are more related to phenol, heparan and chondroitin sulphonation. As a pilot experiment, they were chosen to be cloned and expressed in E. coli after optimization of their nucleotides' sequence to E. coli. This experiments aimed to reveal the role of this class in fucoidan biosynthesis. 


\subsubsection{Material and Methods}

4.3.1.1. Synthetic gene materials

Both E. coli-opt. genes (Esi0032_0064 and Esi0283_0018) were synthesized by Invitrogen GeneArt $^{\circledR}$, as described by Michel, et al. [180,181]. These constructs were delivered as inserts in pMA-T plasmid, as shown in Fig. 62. As mentioned previously in FucTs section (section 4.2.1.1), E. coli JM83 competent cells were transformed and incubated with specified medium (LB supplemented with ampicillin) to produce several genes copies.
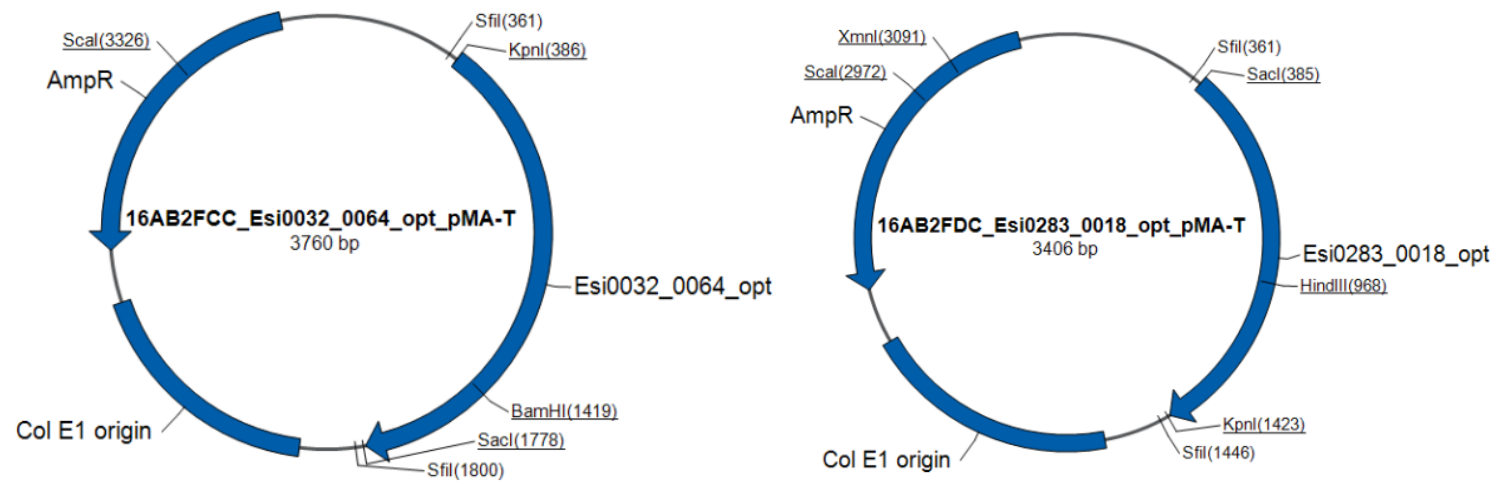

Fig. 62: Cloned E. coli_opt. Esi0032_0064 and Esi0283_0018 in pMA-T plasmid vector

Both DNA constructs were synthesized and delivered by Invitrogen GeneArt ${ }^{\circledR}$, according to Michel, et. al $[180,181]$.

\subsubsection{Cloning by Gibson assembly and sequencing}

As previously described in section 4.2.1.2, E. coli-adapted Esi0032_0064 and Esi0283_0018 genes were cloned inside its multiple cloning site (MCS) specifically between EcoRI and SacI restriction sites of the vector plasmid pASK-IBA $45(+)$. Primers were designed and optimized by the online service provided by New England Biolabs ${ }^{\circledR}$ so as to make genes translation in the same frame of Strep ${ }^{\circledR}$-tag sequence present in the vector plasmid, as demonstrated in Table 17. 
Table 17: Designed primers for cloning of $E$ - coli-adapted Esi0032_0064 and Esi0283_0018 in pASKIBA 45(+), according to NEBuilder ${ }^{\circledR}$ Assembly Tool

\begin{tabular}{|c|c|c|c|}
\hline \multirow{3}{*}{$\begin{array}{c}\text { DNA constructs } \\
\text { pASk-IBA } \\
\text { 45(+)_Esi0032_0064 }\end{array}$} & \multicolumn{2}{|r|}{ Primer } & \multirow{2}{*}{$\begin{array}{c}\begin{array}{c}\text { Annealing } \\
\text { temperature } \\
\left({ }^{\circ} \mathbf{C}\right)\end{array} \\
60.0\end{array}$} \\
\hline & fwd & 5- gcgccgagaccgcggtcccgATGCGTCGTGCACAGGGT -3' & \\
\hline & rev & 5 - ggatcccegggtaccgagctTTAAAAGGCCAGAATAGGCGGTG -3` & \\
\hline \multirow{2}{*}{$\begin{array}{c}\text { pASk-IBA } \\
\text { 45(+)_Esi0283_0018 }\end{array}$} & fwd & 5'-gcgecgagaccgcggtcccgATGGCAGGTAATGCAATG -3` & \multirow[t]{2}{*}{60.0} \\
\hline & rev & 5 - ggatcccegggtaccgagctTTAAAACGGCTGAACCGG -3 & \\
\hline
\end{tabular}

The amplified inserts were afterwards confirmed by gel electrophoresis and PCR products were purified by NucleoSpin ${ }^{\circledR}$ Gel and PCR Clean-up kits (Macherey-Nagel GmbH \& Co. KG) and their concentrations were measured by Nanodrop spectrophotometer.

EcoRI and SacI restriction endonucleases digestion products with PCR products were incubated in a ratio of 1:2 with $10 \mu \mathrm{L}$ NEBuilder ${ }^{\circledR} \mathrm{HiFi}$ DNA Assembly MasterMix in a total volume of $20 \mu \mathrm{L}$ for $15 \mathrm{~min}$ at $50{ }^{\circ} \mathrm{C}$ following the protocol of the provider (New England BioLabs ${ }^{\circledR}$ Inc.) [254]. Recombinant DNA constructs were extracted from culture replicates by NucleoSpin ${ }^{\circledR}$ Plasmid EasyPure (Macherey-Nagel GmbH \& Co. KG), and then digested by XbaI to give single linearized fragments of the original molecular sizes for verification. The constructs with a right gel electrophoresis profile was then sent for sequencing following protocol described in sections 4.2.1.3.

\subsubsection{Transformation of E. coli BL21 (DE3), enzymes expression and purification}

Both sequenced DNA constructs in addition to an empty vector plasmid were applied to transform E. coli BL21 (DE3) by heat shock. A pilot experiment with cultivation, protein expression, detection and purification were performed as previously mentioned in pASK-IBA 45(+)_Esi0021_0026 and Esi0050_0098 transformed E. coli (section 4.2.1.5 and 4.2.1.6).

\subsubsection{Activity assay (Universal sulphotransferase activity kit)}

According to the kit protocol (Catalog \# EA003) described by Bio-Techne GmbH, enzymes' activity was investigated [271,272]. Briefly, purified enzymes were dialyzed in a reaction buffer $(50 \mathrm{mM}$ Tris, $\left.15 \mathrm{mM} \mathrm{MgCl}_{2}, \mathrm{pH} 7.5\right)$. Reaction was initiated by incubation of $25 \mu \mathrm{L}$ of serial dilutions of the enzymes in a $25 \mu \mathrm{L}$ working solution, which was consisted of $10 \mu \mathrm{L} 5 \mathrm{mM}$ GlucNac, $10 \mu \mathrm{L} 1 \mathrm{mM}$ PAPS (as triethylammonium salt) purchased from Sirius fine chemicals (SiChem GmbH) and $5 \mu \mathrm{L}$ $100 \mathrm{ng} \mu \mathrm{L}^{-1}$ coupling phosphatase 3 for $20 \mathrm{~min}$ at $37^{\circ} \mathrm{C}$. Afterwards, $30 \mu \mathrm{L}$ of malachite green $\mathrm{A}$, 
$100 \mu \mathrm{L}$ deionized water and $30 \mu \mathrm{L}$ malachite green B were added, before an incubation step at room temperature again for $20 \mathrm{~min}$. Finally the absorbance was measured at $620 \mathrm{~nm}$ and reaction buffer was used as a blank. 


\subsubsection{Results and Discussion}

Fucan backbone shows different sulphation patterns (e.g., 2- and/or, 3- and/or, 4- sulphated fucan), which is confirmed by the presence of several encoding genes for algal STs [192]. Unlike to fucan synthesis formation site, it is still unclear whether the final fucan sulphonation step is performed in cell cytosol by cytosolic STs and then transported to macroalgal cell wall or inside the cell wall in situ by membrane STs.

\subsubsection{Homology with other STs}

Conserved peptide motifs were observed with alignment of STs_32 and STs_283 amino acids, such as NIAFXKTHKTASTTXAxxLYRYGXRHD and VTVXREPVAHYXSYYYYFLxP which may be related to the enzyme active sites. Moreover, a comparison of the two representatives with the human Galactose-3- $O$-sulfotransferase 2 (G3ST2) revealed 21.9 and 20.9 identity (\%), respectively, as shown in Fig. 63. Lower percentages of homology may be problematic, but the prescene of these motifs proved their putative fucntions.

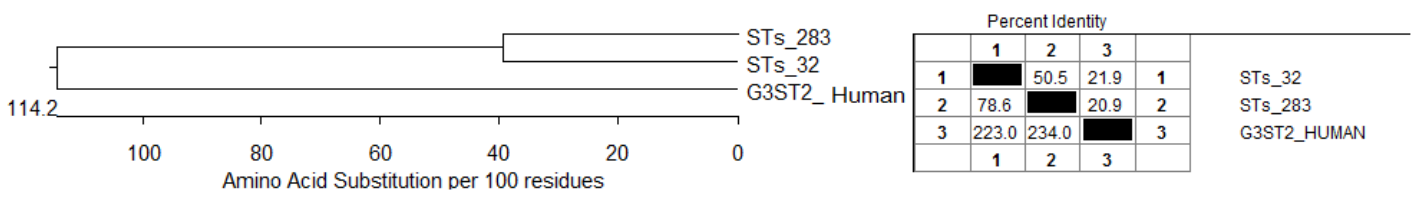

(a)

(b)

Fig. 63: A homology study between algal STs_283, STs_32 and the human Galactose-3-Osulfotransferase 2 (G3ST2)

(a) Phylogenetic tree; (b) percent identity of algal STs_32 and STs_283 with the human enzyme is of 21.9 and $20.9 \%$, respectively. DNASTAR ${ }^{\circledR}$ (Lasergene v7, MegAlign) software and Clustal W algorithm were applied to investigate the different homology studies, including percent identity, phylogenetic tree and motifs in aligned sequences

\subsubsection{PCR of Esi0032_0064 and Esi0283_0018 with overlapping ends}

To design PCR primers with overlapping sequences between plasmid vector pASK-IBA 45(+) and the two DNA fragments for their assembly, the Web Tool of NEBuilder ${ }^{\circledR}$ was applied. Plasmid vector was opened by a double digestion step with EcoRI/SacI; therefore, PCR primers were designed to have an overlapping sequence from plasmid and a gene specific sequence for template priming. Amplified PCR products were verified by agarose gel electrophoresis. As shown in Fig. 64, Esi0032_0064 and Esi0283_0064 were detected at 1386 and 1032 bp, respectively. 


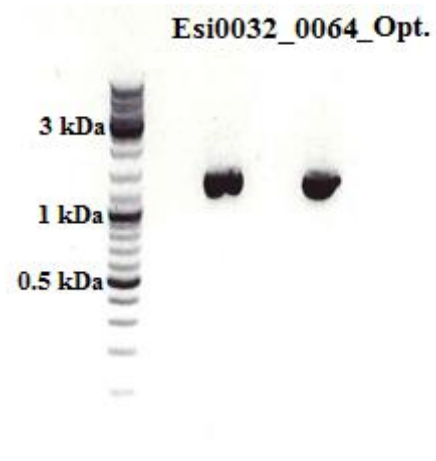

(a)

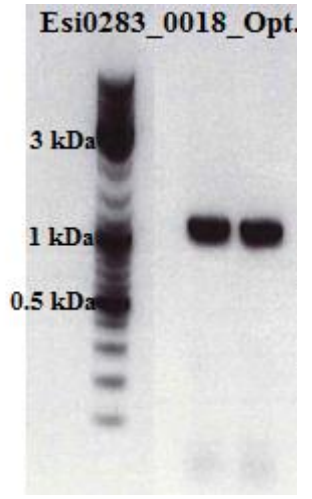

(b)

Fig. 64: Agarose gel electrophoresis of (a) amplified Esi0032_0064 (1386 bp) and (b) Esi0283_0018 $(1032 \mathrm{bp})$ with their overlapping ends from EcoRI/SacI restriction sites of the vector pASKIBA 45(+)

\subsubsection{Gibson assembly and constructs sequencing}

As shown in Fig. 65 and 66, DNA constructs were designed by pDRAW32 DNA analysis Software, as previously performed, and synthesized according to Gibson assembly protocol. Extracted recombinant pASK-IBA 45(+)_Esi0032_0064 construct from replicate 1 and 4 showed after a single digestion by $X b a \mathrm{I}$ a single fragment at 4758 bp. However, several replicates (1-4 and 6) from Esi0283_0018 were detected at 4404 bp. These results were confirmed by lower migration rates of linearized single bands compared with digested vector at $3286 \mathrm{bp}$. 

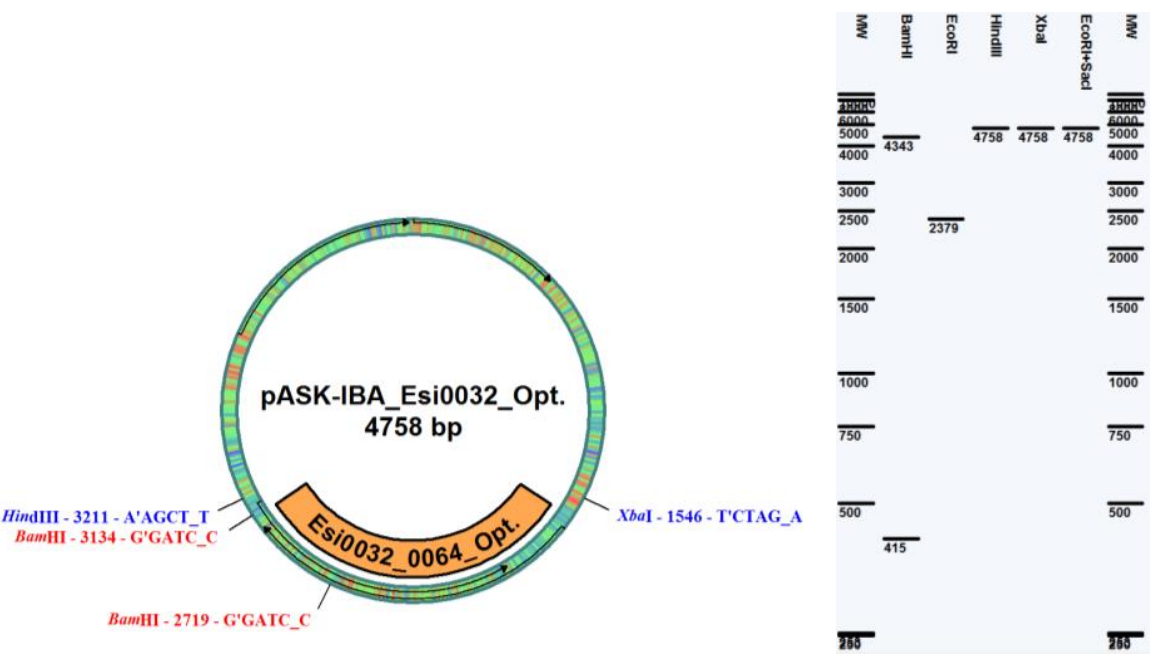

(a)
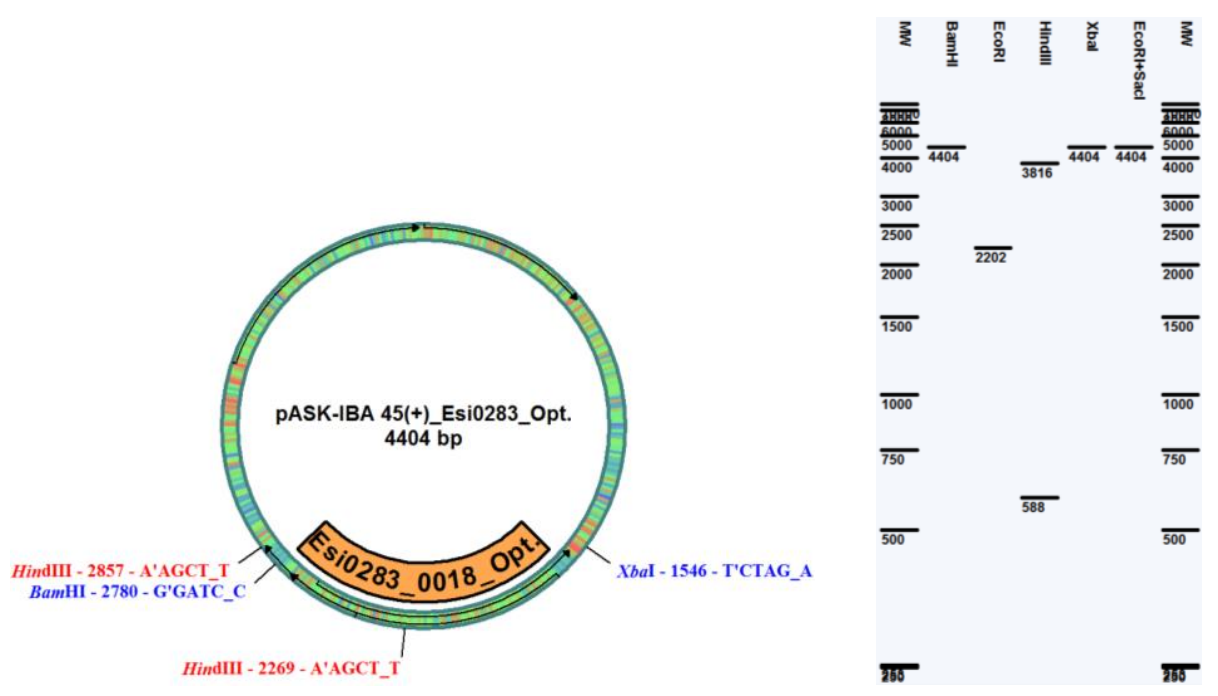

(b)

Fig. 65: Designed pASK-IBA 45(+)_Esi0032_0064 (a) and pASK-IBA 45(+)_Esi0283_0018 (b) templates by pDRAW32 DNA analysis software

Templates showed their sizes in bp and sites of some possible restriction enzymes. 


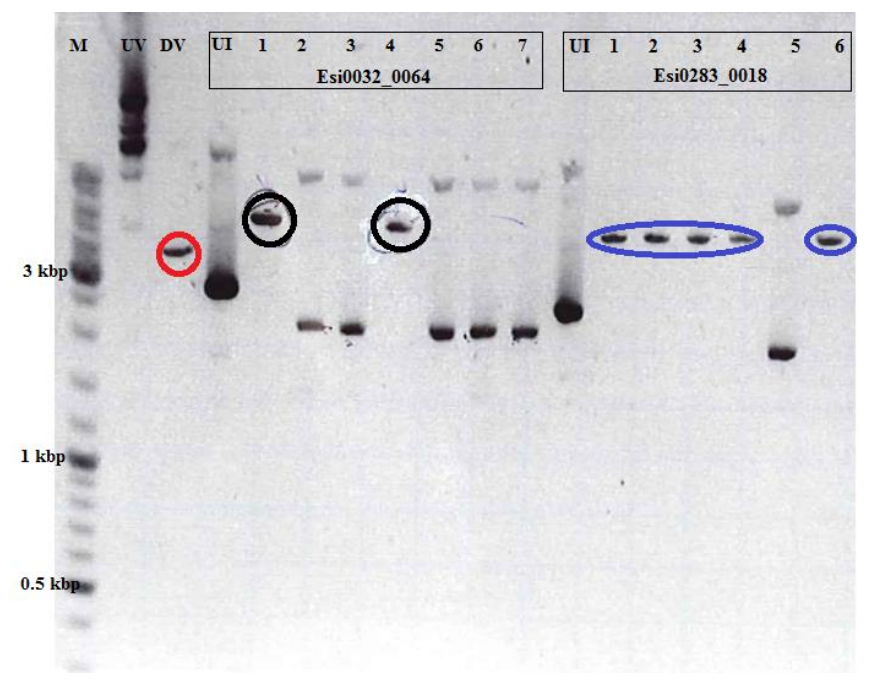

Fig. 66: Agarose gel electrophoresis of extracted DNA constructs; pASK-IBA 45(+)_Esi0032_0064_Opt. and pASK-IBA 45(+)_Esi0283_0018_Opt. after digestion with XbaI

They were compared with undigested empty vector, digested vector and undigested inserts. Constructs 1 and 4 from Esi0032_0064 as a single fragment at 4758 bp and 1-4 and 6 from Esi0283_0018 at 4404 bp showed lower migration rates than digested vector at $3286 \mathrm{bp}$. M= size marker, $\mathrm{UV}=$ undigested empty vector, $\mathrm{DV}=$ digested vector, $\mathrm{UI}=$ undigested insert.

Sequence results were aligned, afterwards, with the designed templates and showed identical sequence in addition to right cloning of synthetic genes in the plasmid vector. Moreover, Strep ${ }^{\circledR}$-tag sequence was inserted correctly as N-terminal in front of the expressed proteins, as demonstrated in Fig. 67. 

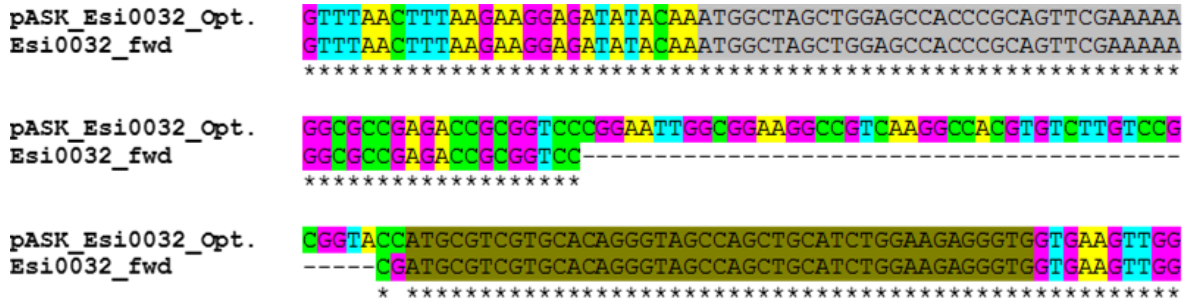

(a)

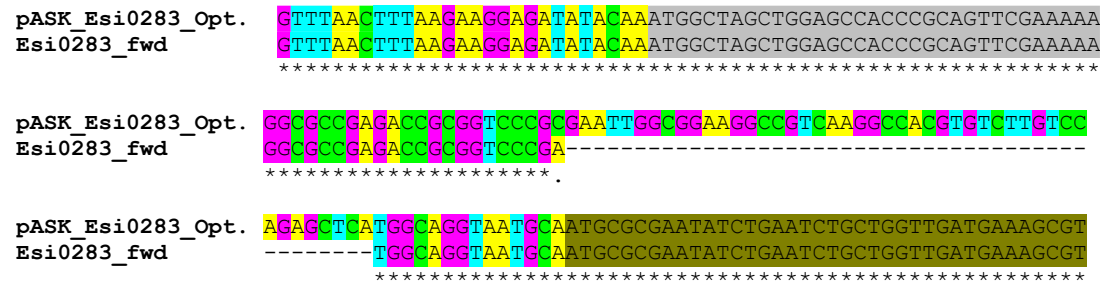

(b)

Fig. 67: Alignment of sequencing results of fwd DNA constructs; pASK-IBA 45(+)_Esi0032_0064_Opt. (a) and pASK-IBA 45(+)_Esi0283_0018_Opt. (b) with the designed templates

The results showed great similarities and insertion of Strep-tag sequence (in grey) as N-terminals of expressed proteins.

\subsubsection{Gene expression and proteins detection by Western blot}

Induction of STs over-expression by AHT from cultivated genetically-engineered E. coli BL21 (DE3) and further steps of proteins purification and detection in eluates were carried out as previously mentioned with FucTs. As demonstrated in Fig. 68 and 69, results confirmed that both STs were expressed as Strep ${ }^{\circledR}$-tag proteins at the right expected molecular sizes with yield of 55.1 $\mu \mathrm{g}$ and $60.5 \mu \mathrm{g}$ from STs_32 and STs_283, respectively, as determined by UV spectrometry at 280 nm.

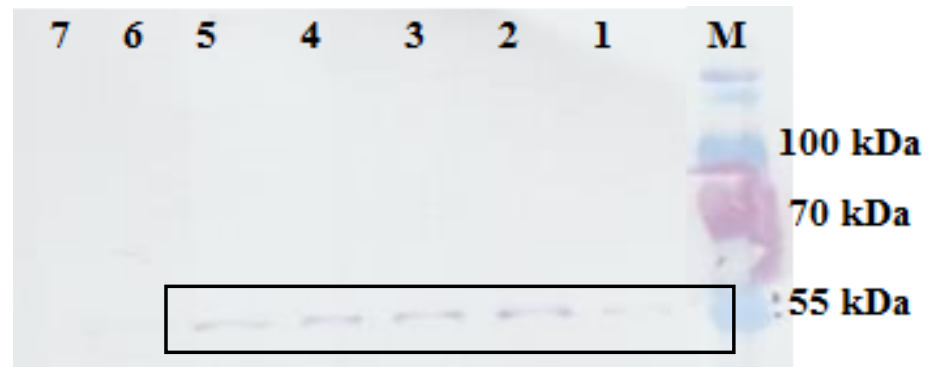

Fig. 68: Western blot of purified heterologusly expressed STs_32 by E. coli BL21 (DE3)

Heterologusly-expressed STs_32 of a molecular size $55.28 \mathrm{kDa}$ was detected in column fractions 25, compared to the size marker. $\mathrm{M}=$ size marker - PageRuler ${ }^{\mathrm{TM}}$ Prestained (Thermo Fisher). 


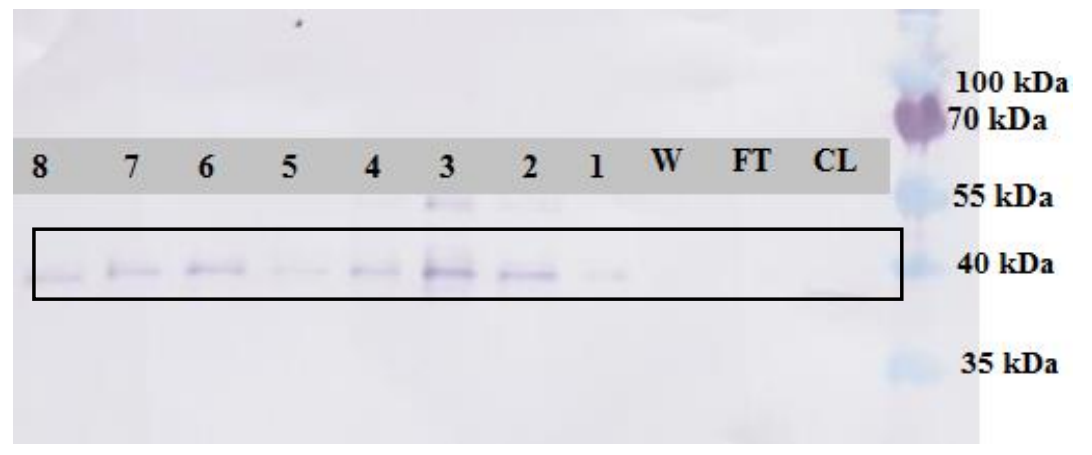

Fig. 69: Western blot of purified heterologusly expressed STs_283 by $E$. coli BL21 (DE3)

Protein of a molecular size $40.58 \mathrm{kDa}$ was detected in column fractions (2-8). $\mathrm{CL}=$ cell lysate, $\mathrm{FT}=$ column flow through, $\mathrm{W}=$ wash.

\subsubsection{Activity assay}

Activity of expressed STs was assessed by their ability to transfer the sulphonate group from the activated sulphonate donor PAPS substrate, according to the protocol established by the kit's provider $[271,272]$. The reaction principle could be summarized as followed in Fig. 70. As a result of sulphonate group removal from PAPS, PAP is produced which and further hydrolyzed by a phosphatase enzyme. The last step is accompanied with the liberation of free phosphate which could be measured colorimetry at $620 \mathrm{~nm}$ by malachite green reagents. 


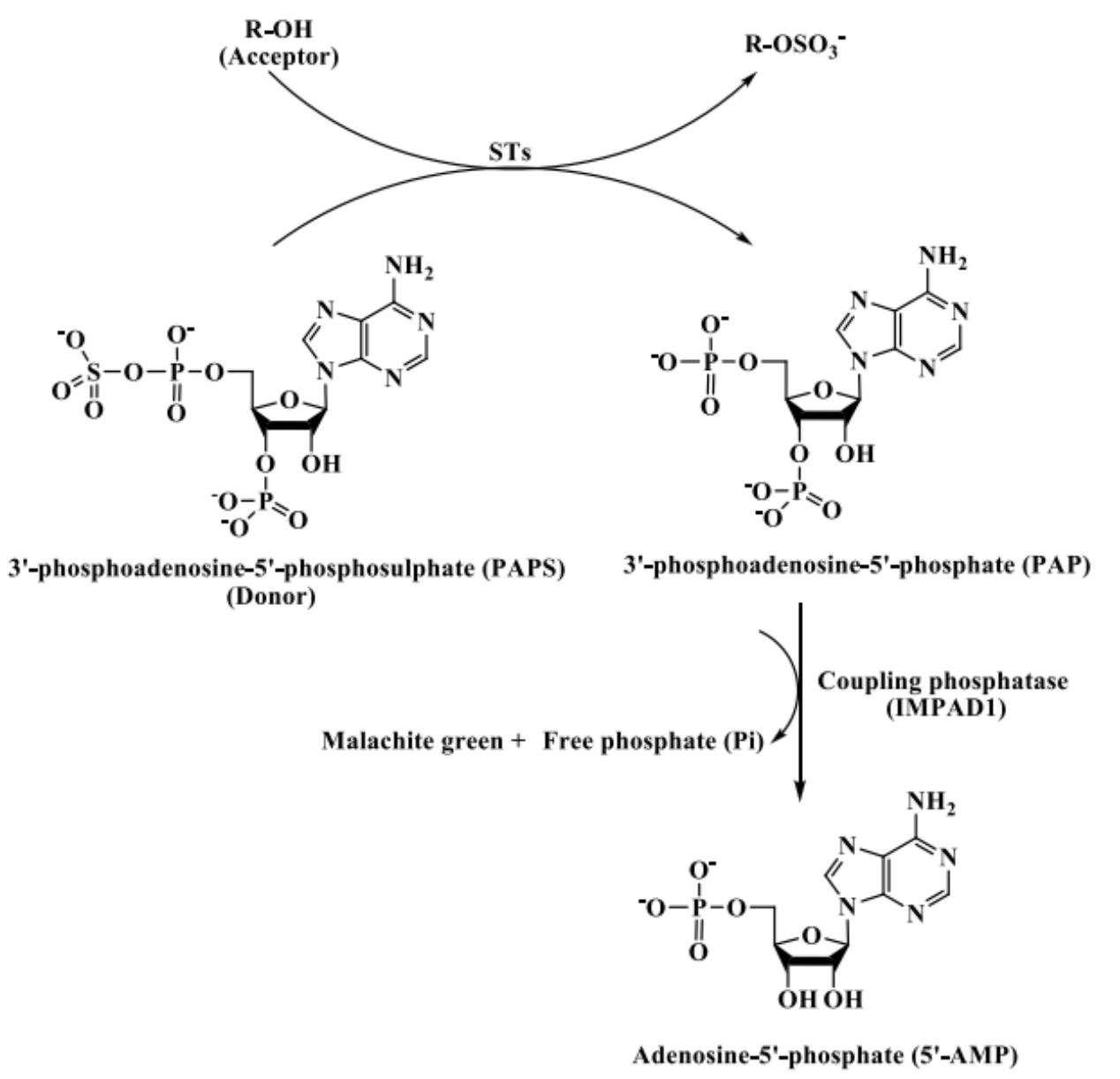

Fig. 70: Principle of the Universal sulphotransferase activity kit as described by the supplier (BioTechne $^{\circledR}$ ) [271,272]

Results, as shown in Fig. 71 a and boproved that STs_32 and STs_283 had a catalytic activity which was directly related with the enzyme concentration. STs_32 and STs_283 recorded similar sulphotransferase activities of 2.38 and $2.29 \mathrm{pmol} \mathrm{min}^{-1} \mu^{-1}$, using Eq. 7, respectively, where; $\mathbf{S}$ is the slope of Fig. 71a and $\mathbf{b}$ and $\mathbf{C F}$ is the conversion factor determined from phosphate concentration calibration curve (see Appendix E: Calibration curves). 


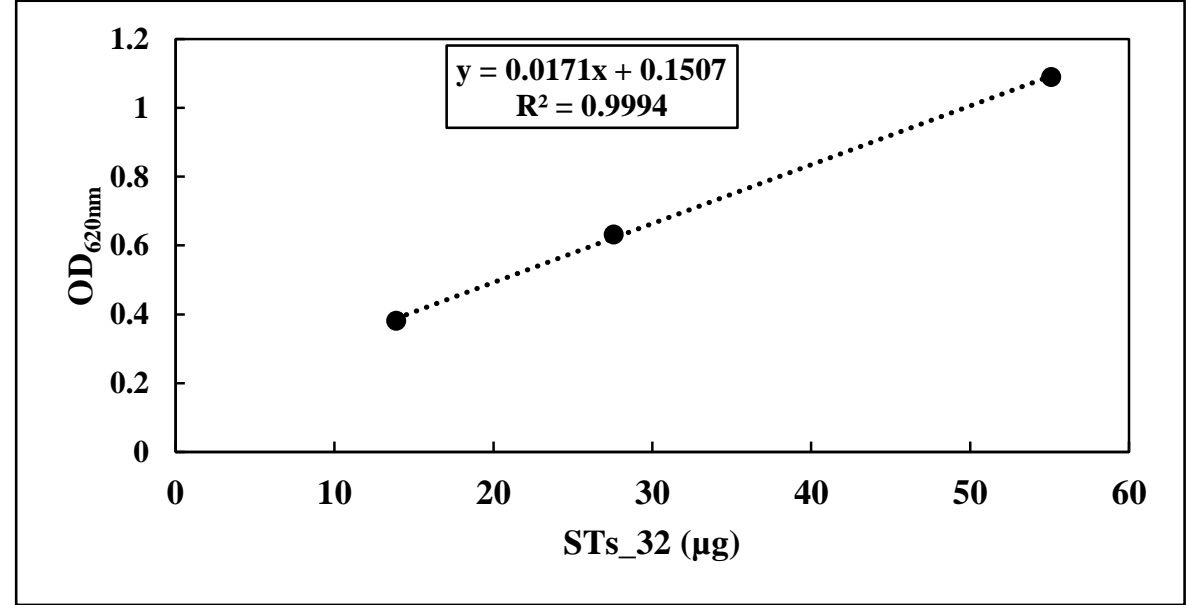

Fig. 71 a: Relationship between purified STs_32 at different concentrations and liberated free phosphate from PAPS detected by malachite green and measured at $620 \mathrm{~nm}$

All absorbance values were subtracted from the background value (reaction buffer).

Specific activity $=\frac{\mathrm{S}\left(\frac{\mathrm{OD}}{\mu \mathrm{g}}\right) \times \mathrm{CF}\left(\frac{\mathrm{pmol}}{\mathrm{OD}}\right)}{\text { Time }(\mathrm{min} .)} \ldots \ldots \ldots \ldots($ Eq. 7$)$

Specific activity of STs_32 $=\frac{0.0171 \times 2784.1}{20}=2.38$ pmol min ${ }^{-1} \mu \mathrm{g}^{-1}$

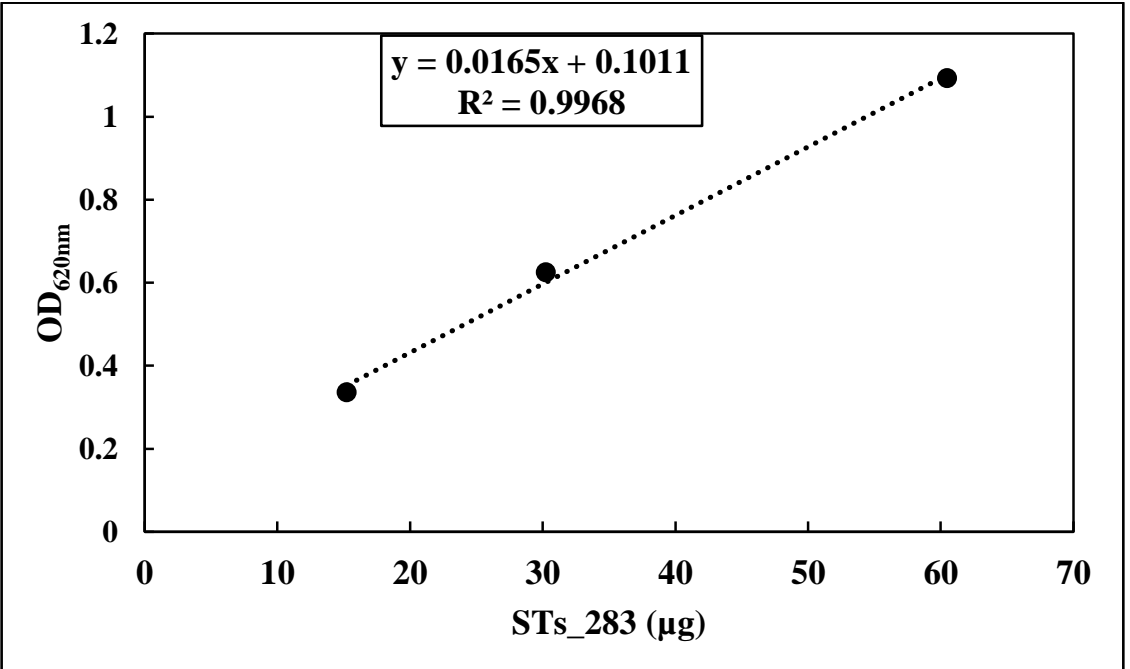

Fig. 71 b: Relationship between purified STs_283 at different concentrations and liberated free phosphate from PAPS detected by malachite green and measured at $620 \mathbf{~ n m}$

All absorbance values were subtracted from the background value (reaction buffer).

Specific activity of STs_283 $=\frac{0.0165 \times 2784.1}{20}=2.29 \mathrm{pmol} \mathrm{min}{ }^{-1} \mu \mathrm{g}^{-1}$ 


\subsection{Conclusion and Prospectives}

Algal FucTs and STs are involved in fucoidan biosynthesis and considered as potential enzyme classes from eukaryotic sources with better similarities with human than bacterial analogues. These enzymes have not been characterized yet and their exact specificity have not been verified. Therefore, heterologous expression of some FucTs and STs in E. coli was performed for the first time.

Overexpressed algal FucTs_50, STS_32 and STs_283 showed a catalytic activity on their donor substrates GDP-L-fucose and PAPS, respectively, as determined by different techniques (e.g., Universal Glycosyltransferase and Sulphotransferase activity kits). These results provided a strong proof for active and correctly-folded enzymes. Nevertheless, overexpression experiments showed proteins with low water solubility and inclusion bodies formation, especially FucTs_21, which affected afterwards on extraction and further purification steps. It might be concluded that these enzymes still needed a post-translational modification which is not present in E. coli. This was confirmed by the presence of a number of serine, threonine and asparagine residues which are possible glycosylation sites. Therefore, lower water solubility might be due to lack of protein glycosylation, which was not possible by a glycosylation machinery-deficient $E$. coli.

Further experiments should be performed to specify acceptor substrates including non-modified substrates (e.g., L-fucose, and GlucNAc) and modified analogues (e.g., $N$-acetyl lactosamine (LacNAc- $t$-Boc type I and II). As seen in Fig. 72, If GlucNAc is considered as an appropriate fucose acceptor, over-expressed putative FucTs_50 should be able to build up an $\alpha-1,3$ glycosidic linkage and produce $\alpha-1,3$ fucosyl $N$-acetyl glucosamine. Product could be detected by different hyphenated spectrometric techniques, such as HPLC-MS [273]. Enzyme kinetics should be addressed applying Michalis-Menten equation to determine $K_{m}$ and $V_{\max }$ kinetic parameters that affect the enzymes' activity in addition to their inhibitors for further characterization. 


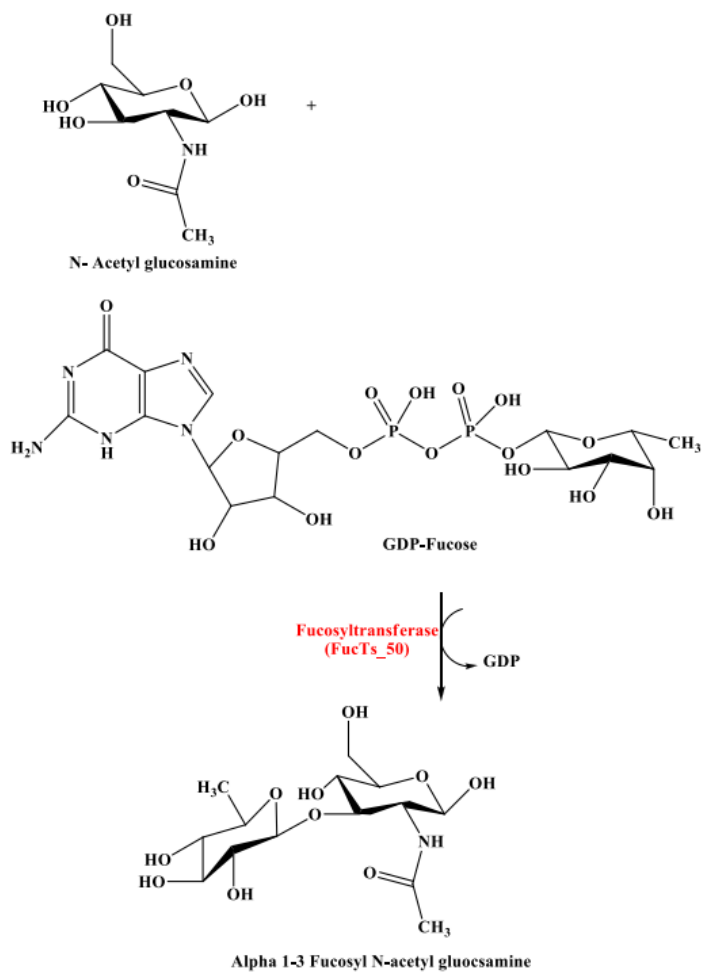

Fig. 72: Simulated fucosylation reaction of GlucNAc catalyzed by heterologously expressed FucTs_50

Furthermore, rate of protein expression and protein water solubility could be increased by their production as GST-fusion proteins or application of eukaryotic expression system with a glycosylation machinery, such as $P$. pastoris.

Moreover, polymer building activity of expressed FucTs should be investigated to be applied in the synthesis of structurally-consistent fucoidan after sulphonation at some positions of polymer backbone with the assist of expressed sulphotransferase (STs). This will help the enzymatic synthesis of a GMP-compliant and eco-friendly product instead of chemical [191] and classical extraction procedures [79]. 


\section{Conclusion}

Increasing the global demands from marine-based products (e.g., fucoidan), heterogeneity, seasonality and negatively consequences on ecosystem showed that novel non-traditional ecofriendly techniques for commercial production of a GMP-compliant product are required. Firstly, it was observed that classical extraction and purification of fucoidan from natural habitat have not been optimized yet which necessitate the emergence of new ideas and techniques for optimization to increase the yield and quality as well. Immobilization of PDD improved fucoidan purification from F. vesiculosus crude extract drastically, in comparison with the recently-established purification by immobilized thiazine dyes. The batch process was performed in only $16 \mathrm{~h}$ instead of $44 \mathrm{~h}$ with thiazine dyes and $3 \mathrm{~h}$ in case of automated process by FPLC. This confirmed that there is a direct relationship between the number of amino groups and reagent sensitivity resulting in a higher percentages of captured fucoidan. Furthermore, purified products demonstrated improved anticoagulant and anti-viral activities, compared with $>95 \%$ pure fucoidan purchased from SigmaAldrich $^{\circledR}$.

Moreover, development of axenic callus-like and protoplast suspension cultures from marine macroalgae as well as cultivation in bioreactors were possible. However, maintenance of cultures growth and induction of fucoidan production were limited by susceptible contamination and undefined nutritional requirements. Induction of hairy root and callus-like growth in wave bag bioreactor were presented for the first time.

In addition, possibility of enzymatic synthesis of marine compounds by heterologous expression of the responsible enzymes was also discussed in the present dissertation showing a novel technique for fucoidan production. Genetically transformed E. coli was able to overexpress some enzymes (e.g., FucTs_50, STs_32 and STs_283) which proved their catalytic activities on their substrates. Moreover, overexpression of algal FucTs and STs might give the opportunity to specify these new category of enzymes and verify bioinformatics predictions.

All of these experiments were performed as potential solutions for production of a fucoidan with a high-quality and low heterogeneity without effects on the global ecosystem. This product can be at this stage investigated clinically to give reliable and consistent results with patients and obey the GMP guidelines. 


\section{Future Outlook}

Further work should continue the optimization trials of cultivation techniques for fast growing cultures of marine organisms at reproducible and controlled conditions (e.g., bioreactor cultivation of hairy root) to assure a constant and homogenous supply of marine-based products such as fucoidan. This field of research needs much work and experiments to follow the advances achieved in terrestrial plants and animals fields.

Overexpression of algal enzymes with their more similarities to human analogues might be applied for synthesis of fucosides and sulphated polysaccharides. Algal enzymes could be seen as a source of enzyme biodiversity for more understanding of their roles in physiological and pathophysiological processes (after investigations of their acceptor substrate specificity) such as synthesis of sialyl Lewis $^{\mathrm{X}}$ antigens and bacterial pathogenicity. In addition, in vitro enzymatic synthesis of fucoidan could be possible with these active enzymes. Incorporation of fucoidan precursors to cultivation medium of a genetically-modified organisms (either E. coli or P. pastoris) might enable the organisms to synthesize fucoidan and incorporate it into its cell wall, secret to the medium or even maintained in site of biosynthesis in cell organelles.

In parallel, traditional extraction and purification techniques should be furtherly optimized especially that there is no standardized procedure. Automated processes, such as developed FPLC purification technique might contribute obtaining a high-yield and pure product without negligible effect on its chemical structure in a time-saving and cheap protocol. Additionally, as a step toward fucoidan approval as a medicament, pharmacokinetic and pharmacodynamics studies should be initiated. For example, accurate and robust analytical techniques which are able to determine its concentrations and recovery (\%) in different synthetic dosage forms should be addressed including TB and Heparin $\operatorname{Red}^{\circledR}$ assay. This study determines robustness of fucoidan measurment in the presence of interferences from drug excipients. 


\section{References}

[1] National Oceanic and Atmospheric Administration, "Marine microbes are tiny, single-celled organisms that live in the ocean and account for more than 98 percent of ocean biomass" [Online]. Available: http://oceanexplorer.noaa.gov/facts/marinemicrobes.html. [Accessed: 21-Nov-2017].

[2] K. R. Arrigo, "Marine microorganisms and global nutrient cycles," Nature, vol. 437, no. 7057, pp. 349-355, 2005.

[3] I. Hamed, F. Özogul, Y. Özogul, and J. M. Regenstein, "Marine bioactive compounds and their health benefits: A review," Comprehensive Reviews in Food Science and Food Safety, vol. 14, no. 4, pp. 446-465, 2015.

[4] C. Tichet, H. K. Nguyen, S. El Yaakoubi, and J. F. Bloch, "Commercial product exploitation from marine microbial biodiversity: Some legal and IP issues: Opinion,” Microbial Biotechnology, vol. 3, no. 5, pp. 507-513, 2010.

[5] X. Wang, H. Yu, R. Xing, and P. Li, "Characterization, preparation, and purification of marine bioactive peptides,” BioMed Research International, vol. 2017, 2017.

[6] Committee on Marine Biotechnology: Biomedical Applications of Marine Natural Products, National Research Council, "Marine biotechnology in the twenty-first century: problems, promise, and products". National academy press, Washington, D.C., 2002.

[7] B. Nijampatnam, S. Dutta, and S. E. Velu, "Recent developments in the isolation, synthesis, and bioactivities of bispyrroloquinone alkaloids of marine origin," Chinese Journal of Natural Medicines, vol. 13, no. 8, pp. 561-577, 2015.

[8] P. M. Murray et al., "Sustainable production of biologically active molecules of marine based origin," New Biotechnology, vol. 30, no. 6, pp. 839-850, 2013.

[9] U. Lindequist, "Marine-derived pharmaceuticals - challenges and opportunities," Biomolecules and Therapeutics, vol. 24, no. 6, pp. 561-571, 2016.

[10] S. Sagar, M. Kaur, and K. P. Minneman, "Antiviral lead compounds from marine sponges," Marine Drugs, vol. 8, no. 10, pp. 2619-2638, 2010.

[11] W. C. Dunlap and Y. Yamamoto, "Small-molecule antioxidants in marine organisms: Antioxidant activity of mycosporine-glycine," Comparative Biochemistry and Physiology - Part B: Biochemistry and, vol. 112, no. 1, pp. 105-114, 1995.

[12] P. Bermejo, E. Piñero, and Á. M. Villar, "Iron-chelating ability and antioxidant properties of phycocyanin isolated from a protean extract of Spirulina platensis," Food Chemistry, vol. 110, no. 2, pp. 436-445, 2008.

[13] A. Asghari, M. Fazilati, A. M. Latifi, H. Salavati, and A. Choopani, "A review on antioxidant properties of Spirulina," Journal of Applied Biotechnology Reports, vol. 3, no. 1, pp. 345-351, 2016.

[14] A. Cumashi et al., "A comparative study of the anti-inflammatory, anticoagulant, antiangiogenic, and antiadhesive activities of nine different fucoidans from brown seaweeds," Glycobiology, vol. 17, no. 5, pp. 541-552, 2007.

[15] Y. González, D. Torres-Mendoza, G. E. Jones, and P. L. Fernandez, "Marine diterpenoids as potential anti-Inflammatory agents," Mediators of Inflammation, vol. 2015, Article ID 263543, 2015.

[16] R. Karthikeyan, S. T. Somasundaram, T. Manivasagam, T. Balasubramanian, and P. Anantharaman, "Hepatoprotective activity of brown alga Padina boergesenii against $\mathrm{CCl}_{4}$ induced oxidative damage in Wistar rats," Asian Pacific Journal of Tropical Medicine, vol. 3, no. 9, pp. 696-701, 2010.

[17] D. G. Nair, R. Weiskirchen, and S. K. Al-Musharafi, "The use of marine-derived bioactive compounds 
as potential hepatoprotective agents," Acta Pharmacologica Sinica, vol. 36, no. 2, pp. 158-170, 2015.

[18] Y. Murti and T. Agrawal, "Marine derived pharmaceuticals-development of natural health products from marine biodiversity," International Journal of ChemTech Research, vol. 2, no. 4, pp. 2198-2217, 2010.

[19] N. Anand, D. Rachel, N. Thangaraju, and P. Anantharaman, "Potential of marine algae (sea weeds) as source of medicinally important compounds," Plant Genetic Resources: Characterisation and Utilisation, vol. 14, no. 4, pp. 303-313, 2016.

[20] G. Schwartsmann, A. B. da Rocha, R. G. S. Berlinck, and J. Jimeno, "Marine organisms as a source of new anticancer agents," The Lancet Oncolog, vol. 2, no. 4, pp. 221-225, 2001.

[21] S. R. Kumar, M. Hosokawa, and K. Miyashita, "Fucoxanthin: A marine carotenoid exerting anticancer effects by affecting multiple mechanisms," Marine Drugs, vol. 11, no. 12, pp. 5130-5147, 2013.

[22] N. Sithranga Boopathy and K. Kathiresan, "Anticancer drugs from marine flora: An overview," Journal of Oncology, vol. 2010, Article ID 214186, 2010.

[23] S. Jiang et al., "Chalcomycins from marine-derived Streptomyces sp. and their antimicrobial activities," Marine Drugs, vol. 15, no. 6, pp. 8-13, 2017.

[24] M. J. Abad, L. M. Bedoya, and P. Bermejo, "Marine compounds and their antimicrobial activities," Fortamex, pp. 1293-1306, 2011.

[25] D. Lee, et al., "Inhibition of Candida albicans isocitrate lyase activity by sesterterpene sulfates from the tropical sponge Dysidea sp.," Bioorganic and Medicinal Chemistry Letters, vol. 18, no. 20, pp. 5377-5380, 2008.

[26] L. Xu, W. Meng, C. Cao, J. Wang, W. Shan, and Q. Wang, "Antibacterial and antifungal compounds from marine fungi," Marine Drugs, vol. 13, no. 6, pp. 3479-3513, 2015.

[27] I. S. Boziaris, "Food ingredients from the marine environment. Marine biotechnology meets food science and technology," Frontiers in Marine Science, vol. 1, no. November, pp. 1-4, 2014.

[28] K. Y. Lee and D. J. Mooney, "Alginate: properties and biomedical applications," Progress in Polymer Science (Oxford), vol. 37, no. 1, pp. 106-126, 2012.

[29] M. Lahaye and C. Rochas, "Chemical structure and physico-chemical properties of agar," Hydrobiologia, vol. 221, pp. 137-148, 1991.

[30] E. Tojo and J. Prado, "Chemical composition of carrageenan blends determined by IR spectroscopy combined with a PLS multivariate calibration method," Carbohydrate Research, vol. 338, no. 12, pp. 1309-1312, 2003.

[31] V. L. Campo, D. F. Kawano, D. B. da Silva, and I. Carvalho, "Carrageenans: Biological properties, chemical modifications and structural analysis - A review," Carbohydrate Polymers, vol. 77, no. 2, pp. 167-180, 2009.

[32] M. Tsuda, et al., "Speradine A, a new pentacyclic oxindole alkaloid from a marine-derived fungus Aspergillus tamarii," Tetrahedron, vol. 59, no. 18, pp. 3227-3230, 2003.

[33] K. K. Tae and J. A. Fuerst, "Diversity of polyketide synthase genes from bacteria associated with the marine sponge Pseudoceratina clavata: Culture-dependent and culture-independent approaches," Environmental Microbiology, vol. 8, no. 8, pp. 1460-1470, 2006.

[34] Y. Zhu, L. Wu, Y. Chen, H. Ni, A. Xiao, and H. Cai, "Characterization of an extracellular biofunctional alginate lyase from marine Microbulbifer sp. ALW1 and antioxidant activity of enzymatic hydrolysates," Microbiological Research, vol. 182, pp. 49-58, 2016.

[35] Q. Wu, M. Zhang, K. Wu, B. Liu, J. Cai, and R. Pan, "Purification and characteristics of fucoidanase obtained from Dendryphiella arenaria TM94," Journal of Applied Phycology, vol. 23, no. 2, pp. 197- 
203, 2011.

[36] H. Malve, "Exploring the ocean for new drug developments: Marine pharmacology," Journal of Pharmacy and Bioallied Sciences, vol. 8, no. 2, pp. 83-91, 2016.

[37] WHO, "InvestigationalPharmaceuticalProductsClinicalTrialsHumansTRS863Annex7.pdf," 1996. [Online]. Available:

http://www.who.int/medicines/areas/quality_safety/quality_assurance/InvestigationalPharmaceutical ProductsClinicalTrialsHumansTRS863Annex7.pdf. [Accessed: 30-Jun-2017].

[38] N. Rhein-Knudsen, M. T. Ale, and A. S. Meyer, "Seaweed hydrocolloid production: An update on enzyme assisted extraction and modification technologies," Marine Drugs, vol. 13, no. 6, pp. 3340$3359,2015$.

[39] A. H. Buschmann, et al., "Seaweed production: overview of the global state of exploitation, farming and emerging research activity," European Journal of Phycology, vol. 52, no. 4, pp. 391-406, 2017.

[40] C. M. Duarte, J. Wu, X. Xiao, A. Bruhn, and D. Krause-Jensen, "Can seaweed farming play a role in climate change mitigation and adaptation?," Frontiers in Marine Science, vol. 4, no. April, 2017.

[41] D. Hurst, T. Børresen, L. Almesjö, F. De Raedemaecker, and S. Bergseth, Marine biotechnology strategic research and innovation roadmap: Insights to the future direction of European marine biotechnology. Marine Biotechnology ERA-NET: Oostende. September 2016.

[42] M. F. de Jesus Raposo, A. M. de Morais, and R. M. de Morais, "Marine polysaccharides from algae with potential biomedical applications," Marine Drugs, vol. 13, no. 5, pp. 2967-3028, 2015.

[43] B. Li, F. Lu, X. Wei, and R. Zhao, "Fucoidan: Structure and bioactivity," Molecules, vol. 13, no. 8, pp. 1671-1695, 2008.

[44] M. Garcia-vaquero, G. Rajauria, O. J. V Doherty, and T. Sweeney, "Polysaccharides from macroalgae: Recent advances, innovative technologies and challenges in extraction and purification," Food Research International, vol. 99, no. September, pp. 1011-1020, 2016.

[45] C. M. Dore, et al., "A sulfated polysaccharide, fucans, isolated from brown algae Sargassum vulgare with anticoagulant, antithrombotic, antioxidant and anti-inflammatory effects," Carbohydrate Polymers, vol. 91, no. 1, pp. 467-475, 2013.

[46] E. Deniaud-Bouët, K. Hardouin, P. Potin, B. Kloareg, and C. Hervé, “A review about brown algal cell walls and fucose-containing sulfated polysaccharides: cell wall context, biomedical properties and key research challenges," Carbohydrate Polymers, vol. 175, pp. 395-408, 2017.

[47] K. Muse, H. Naturelle, and R. Cuvier, "Phylogenetic relationships within the Fucales (Phaeophyceae) assessed by the photosystem I coding psa A sequences," Phycologia, vol. 45, no. September, pp. 512 519, 2006.

[48] M. Wahl, et al., "The responses of brown macroalgae to environmental change from local to global scales: direct versus ecologically mediated effects," Perspectives in Phycology, vol. 2, no. 1, pp. 1129, 2015.

[49] M. R. Irhimeh, J. H. Fitton, and R. M. Lowenthal, "Pilot clinical study to evaluate the anticoagulant activity of fucoidan,” Blood Coagulation \& Fibrinolysis, vol. 20, no. 7, pp. 607-610, 2009.

[50] J. Dürig, T. Bruhn, K.-H. Zurbornl, K. Gutensohn, B. Hans D., and L. Beress, "Anticoagulant fucoidan fractions from Fucus vesiculosus Induce platelet Activation in vitro," Thrombosis Research, vol. 85, no. 6, pp. 479-491, 1997.

[51] H. Young et al., "Anti-inflammatory effects of fucoidan through inhibition of NF- $\mathrm{B}$, MAPK and Akt activation in lipopolysaccharide-induced BV2 microglia cells," Food and Chemical Toxicology, vol. 49, no. 8, pp. 1745-1752, 2011.

[52] S. Palanisamy, M. Vinosha, T. Marudhupandi, P. Rajasekar, and P. N. Marimuthu, "Isolation of 
fucoidan from Sargassum polycystum brown algae: Structural characterization, in vitro antioxidant and anticancer activity," International Journal of Biological Macromolecules, vol. 102, pp. 405-412, 2017.

[53] S. Sinha, A. Astani, T. Ghosh, P. Schnitzler, and B. Ray, "Phytochemistry polysaccharides from Sargassum tenerrimum: Structural features, chemical modification and anti-viral activity," Phytochemistry, vol. 71, no. 2-3, pp. 235-242, 2010.

[54] J. L. Ee, K. H. Ayashi, M. H. Ashimoto, T. N. Akano, and T. H. Ayashi, "Novel antiviral fucoidan from sporophyll of Undaria pinnatifida ( Mekabu )," Chemical Pharmaceutical Bulletin, vol. 52, no. 9, pp. 1091-1094, 2004.

[55] S. Dinesh, T. Menon, L. E. Hanna, V. Suresh, M. Sathuvan, and M. Manikannan, "In vitro anti-HIV1 activity of fucoidan from Sargassum swartzii," International Journal of Biological Macromolecules, vol. 82, pp. 83-88, 2016.

[56] G. Jiao, G. Yu, J. Zhang, and H. S. Ewart, "Chemical structures and bioactivities of sulfated polysaccharides from marine algae," Marine Drugs, vol. 9, pp. 196-223, 2011.

[57] RCR Oliveira, RR Almeida, and TA. Gonçlclves, "A review of plant sulfated polysaccharides and their relations with anticoagulant activities," Journal of Developing Drugs, vol. 5, no. 3, pp. 3-5, 2016.

[58] B. Matsuhiro, "Characterization of a fucoidan from Lessonia vadosa (Phaeophyta) and its anticoagulant and elicitor properties," vol. 42, pp. 235-240, 2008.

[59] M. Baba, R. Snoeck, R. Pauwels, and E. De Clercq, "Sulfated polysaccharides are potent and selective inhibitors of various enveloped viruses, including herpes simplex virus, cytomegalovirus, vesicular stomatitis virus, and human immunodeficiency virus," Antimicrobial Agents and Chemotherapy, vol. 32, no. 11, pp. 1742-1745, 1988.

[60] R. Elizondo-Gonzalez, L. E. Cruz-Suarez, D. Ricque-Marie, E. Mendoza-Gamboa, C. RodriguezPadilla, and L. M. Trejo-Avila, "In vitro characterization of the antiviral activity of fucoidan from Cladosiphon okamuranus against Newcastle Disease Virus," Virology Journal, vol. 9, no. 1, p. 307, 2012.

[61] J. A. Melero and V. Mas, "The Pneumovirinae fusion (F) protein: A common target for vaccines and antivirals," Virus Research, vol. 209, pp. 128-135, 2015.

[62] H. Li et al., "Fucoidan from Fucus vesiculosus suppresses hepatitis B virus replication by enhancing extracellular signal-regulated Kinase activation,” Virology Journal, vol. 14, no. 1, p. 178, 2017.

[63] P. Mandal, C. G. Mateu, K. Chattopadhyay, C. A. Pujol, E. B. Damonte, and B. Ray, "Structural features and antiviral activity of sulphated fucans from the brown seaweed Cystoseira indica," Antiviral Chemistry Chemotherapy, vol. 18, no. 3, pp. 153-162, 2007.

[64] K. K. A. Sanjeewa, J. S. Lee, W. S. Kim, and Y. J. Jeon, "The potential of brown-algae polysaccharides for the development of anticancer agents: An update on anticancer effects reported for fucoidan and laminaran," Carbohydrate Polymers, vol. 177, no. August, pp. 451-459, 2017.

[65] M. T. Ale, J. D. Mikkelsen, and A. S. Meyer, "Important determinants for fucoidan bioactivity: A critical review of structure-function relations and extraction methods for fucose-containing sulfated polysaccharides from brown seaweeds," Marine Drugs, vol. 9, pp. 2106-2130, 2011.

[66] M. C. Rocha De Souza, C. T. Marques, C. M. Guerra Dore, F. R. Ferreira Da Silva, H. A. Oliveira Rocha, and E. L. Leite, "Antioxidant activities of sulfated polysaccharides from brown and red seaweeds," Journal of Applied Phycology, vol. 19, no. 2, pp. 153-160, 2007.

[67] C. Y. Wang, T. C. Wu, S. L. Hsieh, Y. H. Tsai, C. W. Yeh, and C. Y. Huang, "Antioxidant activity and growth inhibition of human colon cancer cells by crude and purified fucoidan preparations extracted from Sargassum cristaefolium," Journal of Food and Drug Analysis, vol. 23, no. 4, pp. 766- 
$777,2015$.

[68] C. Y. Huang, S. J. Wu, W. N. Yang, A. W. Kuan, and C. Y. Chen, “Antioxidant activities of crude extracts of fucoidan extracted from Sargassum glaucescens by a compressional-puffing-hydrothermal extraction process," Food Chemistry, vol. 197, pp. 1121-1129, 2016.

[69] G. Qu, X. Liu, D. Wang, Y. Yuan, and L. Han, "Isolation and characterization of fucoidans from five brown algae and evaluation of their antioxidant activity," Journal of Ocean University of China, vol. 13, no. 5, pp. 851-856, 2014.

[70] W. Mak, N. Hamid, T. Liu, J. Lu, and W. L. White, "Fucoidan from New Zealand Undaria pinnatifida: Monthly variations and determination of antioxidant activities," Carbohydrate Polymers, vol. 95, no. 1, pp. 606-614, 2013.

[71] G. L. Rorrer and D. P. Cheney, "Bioprocess engineering of cell and tissue cultures for marine seaweeds," Aquacultural Engineering, vol. 32, no. 1, pp. 11-41, 2004.

[72] N. Kervarec, G. Michel, T. Tonon, B. Kloareg, and E. Deniaud-boue, "Chemical and enzymatic fractionation of cell walls from Fucales: insights into the structure of the extracellular matrix of brown algae," Annals of Botany, vol. 114, no. 6, pp. 1203-1216, 2014.

[73] H. R. Fletcher, P. Biller, A. B. Ross, and J. M. M. Adams, "The seasonal variation of fucoidan within three species of brown macroalgae," Algal Research, vol. 22, pp. 79-86, 2017.

[74] A. D. Holtkamp, S. Kelly, R. Ulber, and S. Lang, "Fucoidans and fucoidanases - focus on techniques for molecular structure elucidation and modification of marine polysaccharides," Applied Microbiology and Biotechnology, vol. 82, pp. 1-11, 2009.

[75] H. Kylin, “Zur Biochemie der Meeresalgen,” Zeitschrift für Physiologische Chemie, vol. 83, pp. 171197, 1913.

[76] M. T. Ale and A. S. Meyer, "Fucoidans from brown seaweeds: an update on structures, extraction techniques and use of enzymes as tools for structural elucidation," RSC Advances, vol. 22, pp. 81318141, 2013.

[77] P. A. S. Mourão and M. S. Pereira, "Searching for alternatives to heparin sulfated fucans from marine invertebrates," Trends in Cardiovascular Medicine: Discoveries in Cardiology vol. 9, no. 8, pp. 1647$1654,2000$.

[78] S. Li, et al., "4-O-Sulfation in sea cucumber fucodians contribute to reversing dyslipidiaemia caused by HFD," International Journal of Biological Macromolecules, vol. 99, pp. 96-104, 2017.

[79] T. Hahn, S. Lang, R. Ulber, and K. Muffler, "Novel procedures for the extraction of fucoidan from brown algae," Process Biochemistry, vol. 47, no. 12, pp. 1691-1698, 2012.

[80] X. Liu, B. Liu, X. Wei, Z. Sun, and C. Wang, "Extraction, fractionation, and chemical characterisation of fucoidans from the brown seaweed Sargassum pallidum," Czech Journal of Food Sciences, vol. 34, no. 5, pp. 406-413, 2016.

[81] T. I. Imbs, N. M. Shevchenko, and S. V Sukhoverkhov, "Seasonal variations of the composition and structural characteristics of polysaccharides from the brown alga Costaria costata," Chemistry of Natural compounds, vol. 45, no. 6, pp. 661-665, 2009.

[82] E. Percival, "Glucuronoxylofucan, a cell-wall component of Ascophylrum nodosum," Carbohydrate Research, vol. 7, pp. 272-283, 1968.

[83] A. M. Hammed, I. Jaswir, S. Simsek, Z. Alam, and A. Amid, "Enzyme aided extraction of sulfated polysaccharides from Turbinaria turbinata brown seaweed," International Food Research Journal, vol. 24, no. 4, pp. 1660-1666, 2017.

[84] N. Flórez-Fernández, M. López-García, M. J. González-Muñoz, J. M. L. Vilariño, and H. Domínguez, "Ultrasound-assisted extraction of fucoidan from Sargassum muticum," Journal of Applied 
Phycology, vol. 29, no. 3, pp. 1553-1561, 2017.

[85] A. B. Hmelkov, T. N. Zvyagintseva, \& N. M. Shevchenko, A. B. Rasin, and S. P. Ermakova, "Ultrasound-assisted extraction of polysaccharides from brown alga Fucus evanescens. Structure and biological activity of the new fucoidan fractions," Journal of Applied Phycology, 2017.

[86] R. M. Rodriguez-jasso, S. I. Mussatto, L. Pastrana, C. N. Aguilar, and J. A. Teixeira, "Microwaveassisted extraction of sulfated polysaccharides (fucoidan) from brown seaweed," Carbohydrate Polymers, vol. 86, no. 3, pp. 1137-1144, 2011.

[87] Y. Yuan and D. Macquarrie, "Microwave assisted extraction of sulfated polysaccharides (fucoidan) from Ascophyllum nodosum and its antioxidant activity," Carbohydrate Polymers, vol. 129, pp. 101107, 2015.

[88] E. Sinurat and R. Peranginangin, "Purification and characterization of fucoidan from the brown seaweed Sargassum binderi sonder," Squalen Bulletin of Marine \& Fisheries Postharvest \& Biotechnology, vol. 10, no. 2, pp. 79-87, 2015.

[89] T. I. Imbs, A. V. Skriptsova, and T. N. Zvyagintseva, "Antioxidant activity of fucose-containing sulfated polysaccharides obtained from Fucus evanescens by different extraction methods," Journal of Applied Phycology, vol. 27, no. 1, pp. 545-553, 2014.

[90] A. Isnansetyo, F. Nor, L. Lutfia, M. Nursid, R. A. Susidarti, and A. Isnansetyo, "Cytotoxicity of fucoidan from three tropical brown algae against breast and colon cancer cell lines," vol. 9, no. 1, pp. 14-20, 2017.

[91] A. A. El Gamal, "Biological importance of marine algae," Saudi Pharmaceutical Journal, vol. 18, no. 1, pp. 1-25, 2010.

[92] V. Sinniger and J. Tapon-Bretaudière, C. Milien, D. Muller, J. Jozefonvicz and AM. Fischer, “Affinity chromatography of sulphated polysaccharides separatelyfractionated on antithrombin III and heparin cofactor II immobilized onconcanavalin A-Sepharose," Journal of Chromatography, vol. 615, no. 2, pp. 215-223, 1993.

[93] K. Matsumura et al., "Carbohydrate binding specificity of a fucose-specific lectin from Aspergillus oryzae: A novel probe for core fucose," Journal of Biological Chemistry, vol. 282, no. 21, pp. 1570015708, 2007.

[94] T. Hahn et al., "Dye affinity chromatography for fast and simple purification of fucoidan from marine brown algae," Engineering in Life Sciences, vol. 16, no. 1, pp. 78-87, 2016.

[95] A. X. P. D'mello, T. V. Sylvester, V. Ramya, F. P. Britto, P. K. Shetty, and S. Jasphin, "Metachromasia and metachromatic dyes: A review," International Journal of Advanced Health Sciences, vol. 2, no. 10, pp. 12-17, 2016.

[96] Q. Jiao and Q. Liu, "Simple spectrophotometric method for the estimation of algal polysaccharide concentrations," Journal of Agricultural and Food Chemistry, vol. 47, no. 3, pp. 996-998, 1999.

[97] H. S. Soedjak, "Colorimetric determination of carrageenans and other anionic hydrocolloids with methylene blue," Analytical Chemistry, vol. 66, no. 24, pp. 4514-4518, 1994.

[98] T. Hahn et al., "Cationic dye for the specific determination of sulfated polysaccharides," Analytical Letters, vol. 49, no. 12, pp. 1948-1962, 2016.

[99] L. M. Pty., GRAS notification for fucoidan concentrate from Fucus vesiculosus, [Online]. https://www.fda.gov/downloads/Food/IngredientsPackagingLabeling/GRAS/NoticeInventory/ucm52 0974.pdf. [Accessed: 9-March-2018], no. 477. 2016.

[100] B. Harris, "Fucus vesiculosus (Bladder wrack)." [Online]. Available: http://bioweb.uwlax.edu/bio203/2011/harris_benj/habitat.htm. [Accessed: 13-Mar-2018].

[101] D. B. Hermund, "Extraction, characterization and application of antioxidants from the Nordic brown 
alga Fucus vesiculosus," Technical University of Denmark, 2016.

[102] "Fucus vesiculosus Linnaeus." [Online]. Available:

http://www.algaebase.org/search/species/detail/?species_id=Ta869fe1da130ab10. [Accessed: 06Dec-2017].

[103] B. Racionero-Gómez, A. D. Sproson, D. Selby, D. R. Gröcke, H. Redden, and H. C. Greenwell, "Rhenium uptake and distribution in phaeophyceae macroalgae, Fucus vesiculosus," Royal Society Open Science, vol. 3, no. 5, p. 160161, 2016.

[104] M. Dubois, K. A. Gilles, J. K. Hamilton, P. A. Rebers, and F. Smith, "Colorimetric Method for Determination of Sugars and Related Substances," Analytical Chemistry, vol. 28, no. 3, pp. 350-356, 1956.

[105] A. D. Holtkamp, "Isolation, characterisation, modification and application of fucoidan from Fucus vesiculosus," Technical University of Braunschweig, 2009.

[106] U. Warttinger, C. Giese, J. Harenberg, and R. Krämer, "Direct quantification of brown algae-derived fucoidans in human plasma by a fluorescent probe assay," 2016. arXiv:1608.00108 [q-bio.QM]

[107] Z. Dische and L. B. Shettles, "A specific color reaction of methylpentoses and a spectrophotometric micromethod for their determination," The Journal of Biological Chemistry, vol. 175, pp. 595-603, 1948.

[108] T. Hahn, "Extraktion von Fucoidan aus Braunalgen," Technical University of Kaiserslautern, 2012.

[109] H. Szelke, S. Schübel, J. Harenberg, and R. Krämer, "A fluorescent probe for the quantification of heparin in clinical samples with minimal matrix interference.," Chemical communications (Cambridge, England), vol. 46, no. 10, pp. 1667-9, 2010.

[110] Resindion S.r.1., "Relizyme ${ }^{\mathrm{TM}}$ and Sepabeads EC - Ready-to-use Enzyme carriers," Product brochure, 2011.

[111] B. Rühmann, J. Schmid, and V. Sieber, "Fast carbohydrate analysis via liquid chromatography coupled with ultra violet and electrospray ionization ion trap detection in 96-well format," Journal of Chromatography A, vol. 1350, pp. 44-50, 2014.

[112] L. O. Andersson, T. W. Barrowcliffe, E. Holmer, E. A. Johnson, and G. E. Simms "Anticoagulant properties of heparin fractionated by affinity chromatography on matrix-bound antithrombin III and by gel filtration," Thrombosis Research, vol. 9, pp. 575-583, 1976.

[113] A. J. Quick, "The clinical application of the hippuric acid and the prothrombin test," American Journal of Clinical Pathology, vol. 10, pp. 222-233, 1940.

[114] J. Denson, K. W. E., Bonner, "The measurement of heparin: method baseed on the potentiation of anti-factor Xa," Thrombosis et diathesis haemorrhagica, vol. 30, no. 3, pp. 471-479, 1974.

[115] G. Kleymann, and H. O. Werling, "A generally applicable, high-throughput screening-compatible assay to identify, evaluate, and optimize antimicrobial agents for drug therapy," Journal of Biomolecular Screening, vol. 9, no. 7, pp. 578-587, 2004.

[116] F. Boylan, S. Menezes, and G. G. Leita, "Screening of Brazilian plant extracts for antioxidant activity by the use of DPPH free radical method," Phytotherapy Research, vol. 130, no. August, pp. 127-130, 2001.

[117] J. John Peter Paul, "In Vitro anti-oxidant activity of fucoidan extracted from Padina distromatica Hauck (brown seaweed) from Hare Island, Thoothukudi, Tamil Nadu, India," American Journal of Biological and Pharmaceutical Research, vol. 1, no. 3, pp. 151-155, 2014.

[118] S. Park, Y.H., Jang, D.S., Kim, Utilization of Marine Products, $2^{\text {nd }}$ edition. Seoul, South Korea: Hyoungsul Press, 1997.

[119] E. D. Obluchinskaya, G. M. Voskoboinikov, and V. A. Galynkin, "Contents of alginic acid and 
fucoidan in fucus algae of the Barents Sea," Applied Biochemistry and Microbiology., vol. 38, pp. 186-188, 2002.

[120] S. Colin; et al., "Cloning and biochemical characterization of the fucanase FcnA: Definition of a novel glycoside hydrolase family specific for sulfated fucans," Glycobiology, vol. 16, no. 11, pp. 1021-1032, 2006.

[121] V. Descamps; et al., "Isolation and culture of a marine bacterium degrading the sulfated fucans from marine brown algae," Marine Biotechnology, vol. 8, no. 1, pp. 27-39, 2006.

[122] B. de Reviers, "Fucans and alginates without phenolic compounds," Journal of Applied Phycology, vol. 1, no. 1, pp. 75-76, 1989.

[123] U. Warttinger, C. Giese, J. Harenberg, E. Holmer, and R. Krämer, “A fluorescent probe assay (Heparin Red) for direct detection of heparins in human plasma," Analytical and Bioanalytical Chemistry, vol. 408, no. 28, pp. 8241-8251, 2016.

[124] U. Warttinger, and R. Krämer, "Quantification of heparin in complex matrices (including urine) using a mix-and-read fluorescence assay," 2016. arXiv:1611.02482 [q-bio.QM]

[125] M. Rappold, U. Warttinger, and R. Krämer, "A fluorescent probe for glycosaminoglycans applied to the detection of dermatan sulfate by a mix-and-read assay," Molecules, vol. 22, no. 5, pp. 1-11, 2017.

[126] C. Y. Wang and Y. C. Chen, "Extraction and characterization of fucoidan from six brown macroalgae," Journal of Marine Science and Technology (Taiwan), vol. 24, no. 2, pp. 319-328, 2016.

[127] J. H. Fitton, D. N. Stringer, and S. S. Karpiniec, "Therapies from fucoidan: An update," Marine Drugs, vol. 13, no. 9, pp. 5920-5946, 2015.

[128] Aiglent Technologies, "Polymer Molecular Weight Distribution and Definitions of MW Averages," 2015. [Online]. Available: https://www.agilent.com/cs/library/technicaloverviews/Public/59907890EN.pdf. [Accessed: 19-Oct-2017].

[129] J. B. Austin, "A relation between the molecular weights and melting points of organic compounds," Journal of the American Chemical Society, vol. 52, no. 3, pp. 1049-1053, 1930.

[130] E. Sinurat, P. Rosmawaty, and E. Saepudin, “Characterization of fucoidan extracts Binuangeun's brown seaweed," International Journal of Chemical, Environmental and Biological Sciences, vol. 3, no. 4, pp. 329-332, 2015.

[131] N. M. Shevchenko, et al., "Further studies on structure of fucoidan from brown alga Saccharina gurjanovae," Carbohydrate Polymers, vol. 121, pp. 207-216, 2015.

[132] P. Li, Y.-N. Dai, J.-P. Zhang, A.-Q. Wang, and Q. Wei, "Chitosan-alginate nanoparticles as a novel drug delivery system for nifedipine.," International Journal of Biomedical Science, vol. 4, no. 3, pp. 221-228, 2008.

[133] M. B. Hammami, "Partial Thromboplastin Time, Activated," 2015. [Online]. Available: https://emedicine.medscape.com/article/2085837-overview. [Accessed: 23-Oct-2017].

[134] K. Se-kwon, T.-S. Vo, and D.-H. Ngo, Marine Nutraceuticals: Prospects and Perspectives. Ner York: CRC Taylor \& Francis Group, LLC, 2013.

[135] P. A. S. Mourao, "Use of sulfated Fucans as anticoagulant and antithrombotic agents: Future perspectives," Current Pharmaceutical Design, vol. 10, no. 9, pp. 967-981, 2004.

[136] J. Teruya, “Thrombin Time,” 2014. [Online]. Available: https://emedicine.medscape.com/article/2086278-overview.

[137] K. Dobashi, T. Nishino, M. Fujihara, and T. Nagumo, "Isolation and preliminary characterization of fucose-containing sulfated polysaccharides with blood-anticoagulant activity from the brown seaweed Hizikia fusiforme," Carbohydrate Research, vol. 194, no. C, pp. 315-320, 1989.

[138] T. Nishino and T. Nagumo, "Anticoagulant and antithrombin activities of oversulfated fucans," 
Carbohydrate Research, vol. 229, no. 2, pp. 355-362, 1992.

[139] T. Nishino, H. Ura, and T. Nagumo, "The relationship between the sulfate content and the antithrombin activity of an $\alpha(1 \rightarrow 2)$-fucoidan purified from a commercial fucoidan Fraction," Botanica Marina, vol. 38, pp. 187-194, 2009.

[140] T. Nishino, G. Yokoyama, K. Dobashi, M. Fujihara, and T. Nagumo, "Isolation, purification, and characterization of fucose-containing sulphated polysaccharides from the brown seaweed Ecklonia kurome and their blood-anticoagulant activities," Carbohydrate Research, vol. 186, pp. 119-129, 1989.

[141] WHO, "Herpes simplex virus," 2017. [Online]. Available: http://www.who.int/mediacentre/factsheets/fs400/en/. [Accessed: 24-Oct-2017].

[142] W. Saburi, H. M. Ueno, H. Matsui, and H. Mori, "Acidophilic $\beta$-galactosidase from Aspergillus niger AHU7120 with lactose hydrolytic activity under simulated gastric conditions," Journal of Applied Glycoscience, vol. 61, pp. 53-57, 2014.

[143] E. A. Titlyanov and T. V. Titlyanova, "Seaweed cultivation: Methods and problems," Russian Journal of Marine Biology, vol. 36, no. 4, pp. 227-242, 2010.

[144] K. Se-kwon and J. Venkatesan, Handbook of Marine Biotechnology. Springer-Verlag Berlin Heidelberg, 2015.

[145] C. R. K. Reddy, B. Jha, Y. Fujita, and M. Ohno, "Seaweed micropropagation techniques and their potentials: An overview," Journal of Applied Phycology, vol. 20, no. 5, pp. 609-617, 2008.

[146] T. Masakazu, "A simple method of seaweed axenic culture," The Korean Journal of Phycology, vol. 4, no. 2. pp. 183-189, 1989.

[147] N. Kaliaperfumal, "Sea weed biotechnology," Proc. Firts Nayl Semi Mar Biotech, pp. 91-98, 1998.

[148] S. A. Pomponi, "The potential for the marine biotechnology industry," Industry-Driven Changes and Policy Responses, pp. 101-104, 1996.

[149] P. Singh, S. K. Gupta, A. Guldhe, I. Rawat, and F. Bux, "Microalgae isolation and basic culturing techniques," in Handbook of Marine Microalgae: Biotechnology Advances, K. Se-kwon, Ed. Elsevier Inc., 2015, pp. 43-54.

[150] C. Munn, "Microbes in the marine environment," Marine Microbiology Ecology and Applications, pp. 1-24, 2011.

[151] J. Kubanek, P. R. Jensen, P. A. Keifer, M. C. Sullards, D. O. Collins, and W. Fenical, "Seaweed resistance to microbial attack: A targeted chemical defense against marine fungi," Pnas, vol. 100, no. 12, pp. 6916-6921, 2003.

[152] T. Wichard and C. Katsaros, "Phycomorph: Macroalgal development and morphogenesis," Botanica Marina, vol. 60, no. 2, pp. 85-87, 2017.

[153] I. Joint, "Cell-to-cell communication across the prokaryote-eukaryote boundary," Science, vol. 298, no. 5596, p. 1207, 2002.

[154] L. Xuewu and B. Kloareg, "Explant axenisation for tissue culture in marine macroalgae," Chinese Journal of Ocean and Liminology, vol. 10, no. 3, pp. 268-277, 1992.

[155] R. A. Andersen, Ed., Algal Culturing Techniques. MA: Elsevier Academic Press, 2005.

[156] P. Baweja, D. Sahoo, P. García-Jiménez, and R. R. Robaina, "Review: Seaweed tissue culture as applied to biotechnology: Problems, achievements and prospects," Phycological Research, vol. 57, no. 1, pp. 45-58, 2009.

[157] M. Ochoa-Villarreal, et al., "Plant cell culture strategies for the production of natural products," $B M B$ Reports, vol. 49, no. 3, pp. 149-158, 2016.

[158] T.-J. Fu, G. Singh, and W. R. Curtis, Plant Cell and Tissue Culture for the Production of Food 
Ingredients, $1^{\text {st }}$ ed. New York, 1999.

[159] Y. Huang and G. L. Rorrer, "Cultivation of microplantlets derived from the marine red alga Agardhiella subulata in a stirred tank photobioreactor," Biotechnology Progress, vol. 19, pp. 418-427, 2003.

[160] J. G. Modrell, "Bioreactor development and cell culture of the marine macroalgae Porphyra (sp.) and Laminaria saccharina," 1994.

[http://ir.library.oregonstate.edu/concern/graduate_thesis_or_dissertations/44558h09k]

[161] N. Saga, "Isolation of protoplasts from edible seaweeds," The Botanical Magazine, Tokyo, vol. 97, pp. 423-427, 1984.

[162] N. Saga and Y. Sakai, "Isolation of protoplasts from Laminaria paper and Porphyra," Bulletin of the Japanese Society of Scientific Fisheries, vol. 50, no. 6, p. 1085, 1984.

[163] C. R. K. Reddy and Y. Fujita, "Regeneration of plantlets from Enteromorpha (Ulvales, Chlorophyta) protoplasts in axenic culture," Journal of Applied Phycology, vol. 3, no. 3, pp. 265-275, 1991.

[164] B. Moss, “Apical dominance in Fucus vesiculosus," New Phytologist, vol. 64, no. 3, pp. 387-392, 1964.

[165] N. Saga, T. Motomura, and Y. Sakai, "Induction of callus from the marine brown alga Dictyosiphon foeniculaceus," Plant \& Cell Physiology, vol. 23, no. 4, pp. 727-730, 1982.

[166] G. R. Kumar, C. R. K. Reddy, and B. Jha, "Callus induction and thallus regeneration from callus of phycocolloid yielding seaweeds from the Indian coast," Journal of Applied Phycology, vol. 19, no. 1, pp. 15-25, 2007.

[167] P. A. Mooney and J. van Staden, "In vitro plantlet formation and multiple shoot induction in Sargassum heterophyllum," South African Journal of Botany, vol. 51, no. 1, pp. 41-44, 1985.

[168] T. Uji, D. Nanaumi, C. Kawagoe, N. Saga, and K. Miyashita, "Factors influencing the induction of adventitious bud and callus in the brown alga Sargassum horneri (Turner) C. Agardh," Journal of Applied Phycology, vol. 28, no. 4, pp. 2435-2443, 2016.

[169] N. Saga and Y. Sakai, "Axenic tissue culture and callus formation of the marine brown alga Laminaria angustata," Bulletin of the Japanese Society of Scientific Fisheries, vol. 49, no. 10, pp. 1561-1563, 1983.

[170] M. Polne-Fuller and A. Gibor, "Calluses and callus-like growth in seaweeds: Induction and culture," Hydrobiologia, vol. 151-152, no. 1, pp. 131-138, 1987.

[171] W. Huang and Y. Fujita, "Callus indnuction and thallus regeneration in some species of red algae," Phycological Research, vol. 45. pp. 105-111, 1997.

[172] H. Amano and H. Noda, "Effects of plant growth regulators, constituents in the tissue culture organic acids, and sugars of sea lettuce Ulva pertusa," Fisheries Science, vol. 60, no. 4, pp. 449-454, 1994.

[173] G. Rajakrishna Kumar, et al., "Tissue culture and regeneration of thallus from callus of Gelidiella acerosa (Gelidiaies, Rhodophyta)," Phycologia, vol. 43, no. 5, pp. 596-602, 2004.

[174] Qiagen, “AllPrep DNA/ RNA Mini Handbook For simultaneous purification of genomic DNA and total RNA from the same animal," no. November, pp. 1-56, 2005.

[175] A. Koid, W. C. Nelson, A. Mraz, and K. B. Heidelberg, "Comparative analysis of eukaryotic marine microbial assemblages from $18 \mathrm{~s}$ rrna gene and gene transcript clone libraries by using different methods of extraction," Applied and Environmental Microbiology, vol. 78, no. 11, pp. 3958-3965, 2012.

[176] B. H. Nam, H. J. Jin, S. K. Kim, and Y. K. Hong, "Quantitative viability of seaweed tissues assessed with 2,3,5-triphenyltetrazolium chloride," Journal of Applied Phycology, vol. 10, no. 1, pp. 31-36, 1998. 
[177] I. Mussio and A. M. Rusig, "Isolation of protoplasts from Fucus serratus and F. vesiculosus (Fucales, Phaeophyceae): Factors affecting protoplast yield,” Journal of Applied Phycology, vol. 18, no. 6, pp. 733-740, 2006.

[178] I. Mussio and A. M. Rusig, "Morphogenetic responses from protoplasts and tissue culture of Laminaria digitata (Linnaeus) J. V. Lamouroux (Laminariales, Phaeophyta): Callus and thalloid-like structures regeneration," Journal of Applied Phycology, vol. 21, no. 2, pp. 255-264, 2009.

[179] P. J. Harrison and J. A. Berges, "Marine culture media," in Algal Culturing Techniques, 2005, pp. 2133.

[180] N. Sahidin, "Establishment of axenic culture of Kappaphycus alvarezii and optimum culture system for germlings production," Malaysian Fisheries Journal, vol. 13, pp. 33-38, 2014.

[181] S. M. Shishlyannikov, Y. R. Zakharova, N. A. Volokitina, I. S. Mikhailov, D. P. Petrova, and Y. V. Likhoshway, "A procedure for establishing an axinic culture of the diatom Synedra acus subsp. radians (Kütz.) Skabibitsch. from Lake Baikal," Limnology and Oceanography: Methods, vol. 9, pp. 478-484, 2011.

[182] C. G. Bruckner and P. G. Kroth, "Protocols for the removal of bacteria from freshwater benthic diatom cultures," Journal of Phycology, vol. 45, no. 4, pp. 981-986, 2009.

[183] C. A. Leander, D. Porter, and B. S. Leander, "Comparative morphology and molecular phylogeny of aplanochytrids (Labyrinthulomycota)," European Journal of Protistology, vol. 40, no. 4, pp. 317-328, 2004.

[184] G. A. Jones and B. A. Humphrey, " Evaluation of a dehydrogenase assay based on tetrazolium reduction for rapid in vitro estimation of fermentation activity in rumen content," Canadian Journal of Animal Science, vol. 58, pp. 501-511, 1978.

[185] W. Chang and M. Chen, "2,3,5-Triphenyltetrazolium reduction in the viability assay of Ulva fasciata (Chlorophyta) in response to salinity stress," Botanical Bulletin of Academia Sinica, vol. 40, pp. 207212, 1999.

[186] F. P. Altman, "On the oxygen-sensitivity of various tetrazolium salts," Histochemie, vol. 22, no. 3, pp. 256-261, 1970.

[187] M. I. Georgiev, A. I. Pavlov, and T. Bley, "Hairy root type plant in vitro systems as sources of bioactive substances," Applied Microbiology and Biotechnology, vol. 74, no. 6, pp. 1175-1185, 2007.

[188] O. Nilsson and O. Olsson, "Getting to the root: The role of the Agrobacterium rhizogenes rol genes in the formation of hairy roots," Physiologia Plantarum, vol. 100, no. 3, pp. 463-473, 1997.

[189] M. F. Ortiz-Matamoros, T. Islas-Flores, B. Voigt, D. Menzel, F. Baluška, and M. A. Villanueva, "Heterologous DNA uptake in cultured Symbiodinium spp. aided by Agrobacterium tumefaciens," PLoS ONE, vol. 10, no. 7, pp. 1-16, 2015.

[190] J. H. Haas, L. W. Moore, W. Ream, and S. Manulis, "Universal PCR primers for detection of phytopathogenic Agrobacterium strains," Applied and Environmental Microbiology, vol. 61, no. 8, pp. 2879-2884, 1995.

[191] A. Kasai, S. Arafuka, N. Koshiba, D. Takahashi, and K. Toshima, "Systematic synthesis of lowmolecular weight fucoidan derivatives and their effect on cancer cells," Organic \& Biomolecular Chemistry, vol. 13, no. 42, pp. 10556-10568, 2015.

[192] G. Michel, T. Tonon, D. Scornet, J. M. Cock, and B. Kloareg, "The cell wall polysaccharide metabolism of the brown alga Ectocarpus siliculosus. Insights into the evolution of extracellular matrix polysaccharides in Eukaryotes," New Phytologist, vol. 188, pp. 82-97, 2010.

[193] Y. Ren et al., "Biochemical characterization of GDP-L-fucose de novo synthesis pathway in fungus Mortierella alpina," Biochemical and Biophysical Research Communications, vol. 391, pp. 1663- 
$1669,2010$.

[194] P. Mattila, J. Räbinä, S. Hortling, J. Helin, and R. Renkonen, "Functional expression of Escherichia coli enzymes synthesizing GDP-L-fucose from inherent GDP-D-mannose in Saccharomyces cerevisiae," Glycobiology, vol. 10, no. 10, pp. 1041-1047, 2000.

[195] S. G. Byun, M. D. Kim, W. H. Lee, K. J. Lee, N. S. Han, and J. H. Seo, "Production of GDP-L-fucose, L-fucose donor for fucosyloligosaccharide synthesis, in recombinant Escherichia coli," Applied Microbiology and Biotechnology, vol. 74, no. 4, pp. 768-775, 2007.

[196] M. H. Jang, W. H. Lee, S. Y. Shin, N. S. Han, J. H. Seo, and M. D. Kim, "Molecular cloning of the genes for GDP-mannose 4,6-dehydratase and GDP-L-fucose synthetase from Bacteroides thetaiotaomicron," Food Science and Biotechnology, vol. 19, no. 3, pp. 849-855, 2010.

[197] L. Sturla, A. Bisso, D. Zanardi, U. Benatti, A. De Flora, and M. Tonetti, "Expression, purification and characterization of GDP-D-mannose 4,6-dehydratase from Escherichia coli," FEBS Letters, vol. 412, no. 1, pp. 126-130, 1997.

[198] F. X. Sullivan et al., "Molecular cloning of human reconstitution of GDP-fucose biosynthesis in vitro molecular cloning of human GDP-mannose 4,6-dehydratase and reconstitution of GDP-fucose biosynthesis in vitro," The Journal of biological chemistry, vol. 273, no. 14, pp. 8193-8202, 1998.

[199] S. A. Sousa, J. R. Feliciano, P. F. Pinheiro, and J. H. Leitão, "Biochemical and functional studies on the Burkholderia cepacia complex bceN gene, encoding a GDP-D-mannose 4,6-dehydratase," PLoS ONE, vol. 8, no. 2, e56902, 2013.

[200] M. Tonetti, L. Sturla, A. Bisso, U. Benatti, and A. De Flora, "Synthesis of GDP-L-fucose by the human FX protein,” The Journal of Biological Chemistry, vol. 271, no. 44, pp. 27274-27279, 1996.

[201] B. Wu, Y. Zhang, and P. G. Wang, "Identification and characterization of GDP-d-mannose 4,6dehydratase and GDP-1-fucose snthetase in a GDP-1-fucose biosynthetic gene cluster from Helicobacter pylori," Biochemical and Biophysical Research Communications, vol. 285, no. 2, pp. 364-71, 2001.

[202] C. P. Bonin, I. Potter, G. F. Vanzin, and W.-D. Reiter, "The MUR1 gene of Arabidopsis thaliana encodes an isoform of GDP-D-mannose-4,6-dehydratase, catalyzing the first step in the de novo synthesis of GDP-L-fucose," Plant Biology, vol. 94, no. March, pp. 2085-2090, 1997.

[203] C. P. Bonin and W. D. Reiter, "A bifunctional epimerase-reductase acts downstream of the MURI gene product and completes the de novo synthesis of GDP-L-fucose in Arabidopsis," Plant Journal, vol. 21, no. 5, pp. 445-454, 2000.

[204] M. Tonetti, et al., "Paramecium bursaria Chlorella virus 1 encodes two enzymes involved in the biosynthesis of GDP-L-fucose and GDP-D-rhamnose," Journal of Biological Chemistry, vol. 278, no. 24, pp. 21559-21565, 2003.

[205] T. W. Liu, H. Ito, Y. Chiba, T. Kubota, T. Sato, and H. Narimatsu, "Functional expression of Lfucokinase/guanosine 5'-diphosphate-L-fucose pyrophosphorylase from Bacteroides fragilis in Saccharomyces cerevisiae for the production of nucleotide sugars from exogenous monosaccharides," Glycobiology, vol. 21, no. 9, pp. 1228-1236, 2011.

[206] L. Engels and L. Elling, "WbgL: a novel bacterial $\alpha 1,2$-fucosyltransferase for the synthesis of 2'fucosyllactose," Glycobiology, vol. 24, no. 2, pp. 170-178, 2014.

[207] H. Ohashi, C. Wahl, T. Ohashi, L. Elling, and K. Fujiyama, "Effective synthesis of guanosine 5'diphospho- $\beta$ - $l$-galactose using bacterial $l$-Fucokinase/Guanosine 5'-diphosphate- $l$-fucose pyrophosphorylase," Advanced Synthesis \& Catalysis, vol. 359, pp. 4227-4234, 2017.

[208] T. Kotake, S. Hojo, N. Tajima, K. Matsuoka, T. Koyama, and Y. Tsumuraya, “A bifunctional enzyme with L-fucokinase and GDP-L-fucose pyrophosphorylase activities salvages free L-fucose in 
Arabidopsis," Journal of Biological Chemistry, vol. 283, no. 13, pp. 8125-8135, 2008.

[209] H. Wang, et al., "Characterization of an fungal $l$-fucokinase involved in Mortierella alpina GDP-Lfucose salvage pathway," Glycobiology, vol. 26, no. 8, pp. 880-887, 2016.

[210] H. Wang, et al., "Production of GDP-L-fucose from exogenous fucose through the salvage pathway in Mortierella alpina," RSC Advances, vol. 6, no. 52, pp. 46308-46316, 2016.

[211] Z. Ge, N. W. Chan, M. M. Palcic, and D. E. Taylor, "Cloning and heterologous expression of an alpha1,3-fucosyltransferase gene from the gastric pathogen Helicobacter pylori," The Journal of Biological Chemistry, vol. 272, no. 34, pp. 21357-21363, 1997.

[212] C. Dumon, B. Priem, S. L. Martin, A. Heyraud, C. Bosso, and E. Samain, "In vivo fucosylation of lacto- $N$-neotetraose and lacto- $N$-neohexaose by heterologous expression of Helicobacter pylori $\alpha-1,3$ fucosyltransferase in engineered Escherichia coli," Glycoconjugate Journal, vol. 18, no. 6, pp. 465474, 2001.

[213] P. F. Gallet, et al., "Heterologous expression of an engineered truncated form of human Lewis fucosyltransferase (Fuc-TIII) by the methylotrophic yeast Pichia pastoris," Glycobiology, vol. 8, no. 9, pp. 919-925, 1998.

[214] J. S. Bondili, et al., "Molecular cloning and heterologous expression of $\beta 1,2$-xylosyltransferase and core $\alpha 1,3$-fucosyltransferase from maize," Phytochemistry, vol. 67, no. 20, pp. 2215-2224, 2006.

[215] M. Malissard, S. Zeng, and E. G. Berger, "Expression of functional soluble forms of human beta-1,4galactosyltransferase I, alpha-2,6-sialyltransferase, and alpha-1, 3-fucosyltransferase VI in the methylotrophic yeast Pichia pastoris," Biochemical and Biophysical Research Communications, vol. 267, no. 1, pp. 169-173, 2000.

[216] A. Bastida, A. Fernández-Mayoralas, R. Gómez Arrayás, F. Iradier, J. C. Carretero, and E. GarcíaJunceda, "Heterologous over-expression of $\alpha$-1,6-fucosyltransferase from Rhizobium sp.: Application to the synthesis of the trisaccharide $\beta$-D-GlcNAc $(1 \rightarrow 4)-[\alpha$-L-Fuc- $(1 \rightarrow 6)]-\mathrm{D}-\mathrm{GlcNAc}$, study of the acceptor specificity and evaluation of polyhydroxylated indolizidines as inhibitors," Chemistry - A European Journal, vol. 7, no. 11, pp. 2390-2397, 2001.

[217] J. Shao, M. Li, Q. Jia, Y. Lu, and P. G. Wang, "Sequence of Escherichia coli O128 antigen biosynthesis cluster and functional identification of an $\alpha$-1,2-fucosyltransferase," FEBS Letters, vol. 553, no. 1-2, pp. 99-103, 2003.

[218] W. Yi, et al., "Escherichia coli O86 O-antigen biosynthetic gene cluster and stepwise enzymatic synthesis of human blood group B antigen tetrasaccharide," Journal of the American Chemical Society, vol. 127, no. 7, pp. 2040-2041, 2005.

[219] N. Pettit, T. Styslinger, Z. Mei, W. Han, G. Zhao, and P. G. Wang, "Characterization of WbiQ: An $\alpha$ 1,2-fucosyltransferase from Escherichia coli O127:K63(B8), and synthesis of H-type 3 blood group antigen," Biochemical and Biophysical Research Communications, vol. 402, no. 2, pp. 190-195, 2010.

[220] C. Albermann, W. Piepersberg, and U. F. Wehmeier, "Synthesis of the milk oligosaccharide 2'fucosyllactose using recombinant bacterial enzymes," Carbohydrate Research, vol. 334, no. 2, pp. 97103, 2001.

[221] G. Wang, P. G. Boulton, N. W. C. Chan, M. M. Palcic, and D. E. Taylor, "Novel Helicobacter pylori a1,2- fucosyltransferase, a key enzyme in the synthesis of Lewis antigens," Microbiology, vol. 145, no. 11, pp. 3245-3253, 1999.

[222] D. B. Stein, Y. N. Lin, and C. H. Lina, "Characterization of Helicobacter pylori $\alpha 1,2-$ fucosyltransferase for enzymatic synthesis of tumor-associated antigens," Advanced Synthesis and Catalysis, vol. 350, no. 14-15, pp. 2313-2321, 2008.

[223] Y. W. Chin, J. Y. Kim, J. H. Kim, S. M. Jung, and J. H. Seo, "Improved production of 2'-fucosyllactose 
in engineered Escherichia coli by expressing putative $\alpha$-1,2-fucosyltransferase, WcfB from Bacteroides fragilis," Journal of Biotechnology, vol. 257, pp. 192-198, 2017.

[224] L. M. Bidwell, E. M. J. Gillam, A. Gaedigk, X. Zhu, D. Grant, and M. E. McManus, "Bacterial expression of two human aryl sulfotransferases," Chemico-Biological Interactions, vol. 109, no. 1-3, pp. 137-141, 1998.

[225] M. C. Baek, K. H. Choi, T. G. Oh, D. H. Kim, and E. C. Choi, "Overexpression of arylsulfate sulfotransferase as fusion protein with glutathione S-transferase," Protein Expression and Purification, vol. 11, no. 3, pp. 257-262, 1997.

[226] I. Ayuso-Fernández, M. A. Galmés, A. Bastida, and E. García-Junceda, "Aryl sulfotransferase from Haliangium ochraceum: A versatile tool for the sulfation of small molecules," ChemCatChem, vol. 6, no. 4, pp. 1059-1065, 2014.

[227] J. R. Myette, Z. Shriver, J. Liu, G. Venkataraman, R. Rosenberg, and R. Sasisekharan, "Expression in Escherichia coli, purification and kinetic characterization of human heparan sulfate 3-Osulfotransferase-1," Biochemical and biophysical research communications, vol. 290, no. 4, pp. 1206$13,2002$.

[228] X. Zhou, K. Chandarajoti, T. Q. Pham, R. Liu, and J. Liu, "Expression of heparan sulfate sulfotransferases in Kluyveromyces lactis and preparation of 3'-phosphoadenosine-5'phosphosulfate," Glycobiology, vol. 21, no. 6, pp. 771-780, 2011.

[229] A. T. Do, E. Smeds, D. Spillmann, and M. Kusche-Gullberg, "Overexpression of heparan sulfate 6$O$-sulfotransferases in human embryonic kidney 293 cells results in increased $N$-acetylglucosaminyl 6-O-sulfation," Journal of Biological Chemistry, vol. 281, no. 9, pp. 5348-5356, 2006.

[230] K. Kamimura, et al., "Drosophila heparan sulfate 6- $O$-sulfotransferase (dHS6ST) gene. Structure, expression, and function in the formation of the tracheal system," Journal of Biological Chemistry, vol. 276, no. 20, pp. 17014-17021, 2001.

[231] A. S. Saribaş, et al., "Production of $N$-sulfated polysaccharides using yeast-expressed $N$ deacetylase/N-sulfotransferase-1 (NDST-1)," Glycobiology, vol. 14, no. 12, pp. 1217-1228, 2004.

[232] D. S. Pikas, I. Eriksson, and L. Kjellén, "Overexpression of different isoforms of glucosaminyl $N$ deacetylase/ $N$-sulfotransferase results in distinct heparan sulfate $N$-sulfation patterns," Biochemistry, vol. 39, no. 15, pp. 4552-4558, 2000.

[233] J. Presto, et al., "Heparan sulfate biosynthesis enzymes EXT1 and EXT2 affect NDST1 expression and heparan sulfate sulfation," Proceedings of the National Academy of Sciences, vol. 105, no. 12, pp. 4751-4756, 2008.

[234] A. Deligny, et al., "NDST2 ( $N$-deacetylase/ $N$-sulfotransferase-2) enzyme regulates heparan sulfate chain length," Journal of Biological Chemistry, vol. 291, no. 36, pp. 18600-18607, 2016.

[235] Y. Sakakibara, et al., "Molecular cloning, expression, and characterization of novel human SULT1C sulfotransferases that catalyze the sulfonation of $N$-hydroxy-2-acetylaminofluorene," The Journal of Biological Chemistry, vol. 273, no. 51, pp. 33929-35, 1998.

[236] N. Hehonah, et al., "Molecular cloning, expression, localisation and functional characterisation of a rabbit SULT1C2 sulfotransferase," International Journal of Biochemistry and Cell Biology, vol. 31, no. 8, pp. 869-882, 1999.

[237] T. Shimohira, K. Kurogi, T. Hashiguchi, M. C. Liu, M. Suiko, and Y. Sakakibara, "Regioselective production of sulfated polyphenols using human cytosolic sulfotransferase-expressing Escherichia coli cells," Journal of Bioscience and Bioengineering, vol. 124, no. 1, pp. 84-90, 2017.

[238] X. Chen, Y.-S. Yang, Y. Zheng, B. M. Martin, M. W. Duffel, and W. B. Jakoby, "Tyrosine-Ester Sulfotransferase from Rat Liver: Bacterial Expression and Identification," Protein Expression and 
Purification, vol. 3, pp. 421-426, 1992.

[239] Y. Sakakibara, et al., "Purification, characterization, and molecular cloning of a novel rat liver Dopa/tyrosine sulfotransferase," vol. 270, no. 51, pp. 30470,30478, 1995.

[240] R. Dajani, et al., "Kinetic properties of human dopamine sulfotransferase (SULT1A3) expressed in prokaryotic and eukaryotic systems: Comparison with the recombinant enzyme purified from Escherichia coli," Protein Expression and Purification, vol. 16, no. 1, pp. 11-18, 1999.

[241] B. I. Cantarel, P. M. Coutinho, C. Rancurel, T. Bernard, V. Lombard, and B. Henrissat, "The Carbohydrate-Active EnZymes database (CAZy): An expert resource for glycogenomics," Nucleic Acids Research, vol. 37, no. SUPPL. 1, pp. 233-238, 2009.

[242] L. L. Lairson, B. Henrissat, G. J. Davies, and S. G. Withers, "Glycosyltransferases: Structures, Functions, and mechanisms," Annual Review of Biochemistry, vol. 77, no. 1, pp. 521-555, 2008.

[243] B. Ma, J. L. Simala-Grant, and D. E. Taylor, "Fucosylation in prokaryotes and eukaryotes," Glycobiology, vol. 16, no. 12, pp. 158R-184R, 2006.

[244] L. Muinelo-Romay, S. Villar-Portela, E. Cuevas, E. Gil-Martin, and A. Fernandez-Briera, "Identification of alpha $(1,6)$ fucosylated proteins differentially expressed in human colorectal cancer," BMC Cancer, vol. 11, no. 1, p. 508, 2011.

[245] G. Fabini, A. Freilinger, F. Altmann, and I. B. H. Wilson, "Identification of core $\alpha 1,3$-fucosylated glycans and cloning of the requisite fucosyltransferase cDNA from Drosophila melanogaster: Potential basis of the neural anti-horseradish peroxidase epitope," Journal of Biological Chemistry, vol. 276, no. 30, pp. 28058-28067, 2001.

[246] H. Kajiwara, M. Toda, T. Mine, H. Nakada, and T. Yamamoto, "Isolation of fucosyltransferaseproducing bacteria from marine environments," Microbes and Environments, vol. 27, no. 4, pp. 515518, 2012.

[247] P. A. Prieto, et al., "Expression of human H-type $\alpha 1$,2-fucosyltransferase encoding for blood group H (O) antigen in chinese hamster ovary cells," vol. 272, no. 4, pp. 2089-2097, 1997.

[248] C. Joly, R. Leonard, A. Maftah, and C. Riou-Khamlichi, "alpha4-Fucosyltransferase is regulated during flower development: increases in activity are targeted to pollen maturation and pollen tube elongation," Journal of Experimental Botany, vol. 53, no. 373, pp. 1429-1436, 2002.

[249] X. Yang, S. Liu, and Q. Yan, "Role of fucosyltransferase IV in epithelial-mesenchymal transition in breast cancer cells," Cell Death and Disease, vol. 4, no. 7, pp. e735-9, 2013.

[250] E. Miyoshi, et al., "Fucosylation is a promising target for cancer diagnosis and therapy," Biomolecules, vol. 2, no. 4, pp. 34-45, 2012.

[251] A. P. Moran, "Relevance of fucosylation and Lewis antigen expression in the bacterial gastroduodenal pathogen Helicobacter pylori," Carbohydrate Research, vol. 343, no. 12, pp. 1952-1965, 2008.

[252] G. Michel, "Online Resource for Community Annotation of Eukaryotes." [Online]. Available: http://bioinformatics.psb.ugent.be/orcae/annotation/Ectsi/current/Esi0050_0098. [Accessed: 20-Nov2015].

[253] G. Michel, “Online Resource for Community Annotation of Eukaryotes.” [Online]. Available: http://bioinformatics.psb.ugent.be/orcae/annotation/Ectsi/current/Esi0021_0026. [Accessed: 20-Nov2015].

[254] New England Biolabs, "NEBuilder HiFi DNA Assembly Master Mix," Instruction Manual, vol. 1, no. 10, pp. 1-23, 2014.

[255] New England Biolabs, "Gibson Assembly ${ }^{\mathrm{TM}}$ Cloning Kit," Instruction Manual, vol. 3.2, 2017.

[256] IBABioTAGnology, "pASK-IBA 45(+) Data Sheet," vol. 402, pp. 1-8, 2005.

[257] IBA, "Expression and purification of proteins using 6x Histidine -tag A comprehensive manual," IBA 
Solutions for Life Sciences, no. October, pp. 1-36, 2012.

[258] IBABioTAGnology, “Strep-tag ${ }^{\circledR}$ detection in Western blots," Instruction Manual, vol. PR07-0012, p. 7, 2016.

[259] Z. L. Wu, C. M. Ethen, B. Prather, M. MacHacek, and W. Jiang, "Universal phosphatase-coupled glycosyltransferase assay," Glycobiology, vol. 21, no. 6, pp. 727-733, 2011.

[260] C. Wahl, D. Hirtz, and L. Elling, "Multiplexed capillary electrophoresis as analytical tool for fast optimization of multi-enzyme cascade reactions-synthesis of nucleotide sugars: Dedicated to Prof. Dr. Vladimir Křen on the occasion of his $60^{\text {th }}$ birthday," Biotechnology Journal, vol. 11, no. 10, pp. 1298-1308, 2016.

[261] NCBI, "NCBI blast protein sequence.” [Online]. Available: https://blast.ncbi.nlm.nih.gov/Blast.cgi\#563472779. [Accessed: 02-Feb-2018].

[262] NCBI, "NCBI blast protein sequence.” [Online]. Available: https://blast.ncbi.nlm.nih.gov/Blast.cgi. [Accessed: 02-Feb-2018].

[263] C. Dumon, E. Samain, and B. Priem, "Assessment of the two Helicobacter pylori a-1,3fucosyltransferase ortholog genes for the large-scale synthesis of lewisx human milk oligosaccharides by metabolically engineered Escherichia coli," Biotechnology Progress, vol. 20, no. 2, pp. 412-419, 2004.

[264] T. De Vries, R. M. A. Knegtel, E. H. Holmes, and B. A. Macher, "Fucosyltransferases: structure/function studies," Glycobiology, vol. 11, no. 10, pp. 119-128, 2001.

[265] I. Martinez-Duncker, R. Mollicone, J. J. Candelier, C. Breton, and R. Oriol, “A new superfamily of protein- $O$-fucosyltransferases, $\alpha 2$-fucosyltransferases, and $\alpha 6$-fucosyltransferases: Phylogeny and identification of conserved peptide motifs," Glycobiology, vol. 13, no. 12, pp. 1-5, 2003.

[266] New England Biolabs, "Gibson Assembly ${ }^{\circledR}$." [Online]. Available:

https://www.neb.com/applications/cloning-and-synthetic-biology/dna-assembly-and-cloning/gibsonassembly.

[267] M. M. Bradford, "A rapid and sensitive method for the quantitation of microgram quantities of protein utilizing the principle of protein-dye binding," Analytical Biochemistry, vol. 72, no. 1-2, pp. 248-254, 1976.

[268] E. Chapman, M. D. Best, S. R. Hanson, and C. H. Wong, "Sulfotransferases: Structure, mechanism, biological activity, inhibition, and synthetic utility," Angewandte Chemie - International Edition, vol. 43, no. 27, pp. 3526-3548, 2004.

[269] G. Michel, "Online resource for community annotation of eukaryotes." [Online]. Available: http://bioinformatics.psb.ugent.be/orcae/annotation/Ectsi/current/Esi0032_0064. [Accessed: 16-Oct2015].

[270] G. Michel, "Online resource for community annotation of eukaryotes." [Online]. Available: http://bioinformatics.psb.ugent.be/orcae/annotation/Ectsi/current/Esi0283_0018. [Accessed: 16-Oct2015].

[271] B. Prather, C. M. Ethen, M. MacHacek, and Z. L. Wu, "Golgi-resident PAP-specific 3'-phosphatasecoupled sulfotransferase assays," Analytical Biochemistry, vol. 423, no. 1, pp. 86-92, 2012.

[272] Z. L. Wu, "Phosphatase-coupled universal kinase assay and kinetics for first-order-rate coupling reaction," PLoS ONE, vol. 6, no. 8, p. e23172, 2011.

[273] T. Fischöder, D. Laaf, C. Dey, and L. Elling, "Enzymatic synthesis of $N$-acetyllactosamine (LacNAc) type 1 oligomers and characterization as multivalent galectin ligands," Molecules, vol. 22, no. 8, pp. $1-15,2017$.

[274] M. Kovacheva, "Kultivierung von Makroalgen,” TU Kaiserslautern, 2012. 



\section{Appendix}

\section{Appendix A}

\section{Chemical structures of some marine-derived bioactive compounds}<smiles>COC1=C(NCC(=O)O)CC(C)(CO)CC1=O</smiles>

Mycosporine-Gly

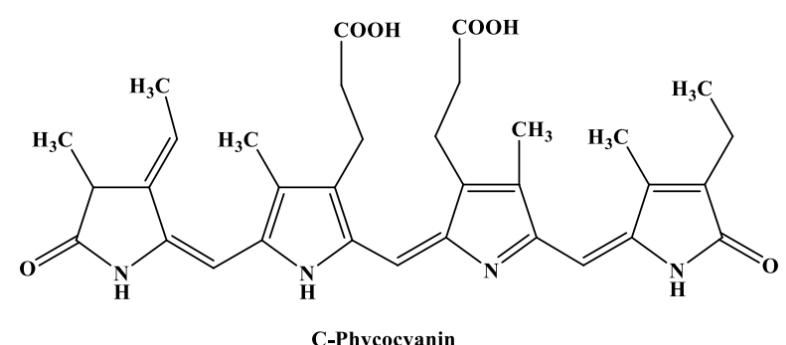

C-Phycocyanin

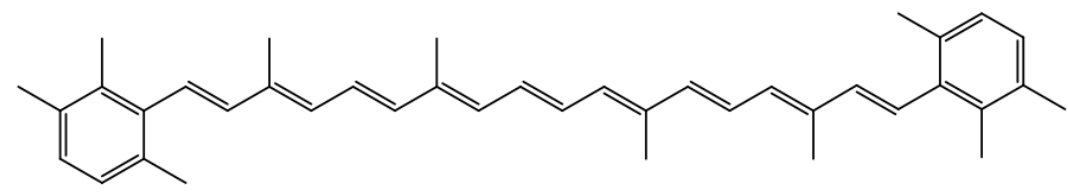

Carotenoid

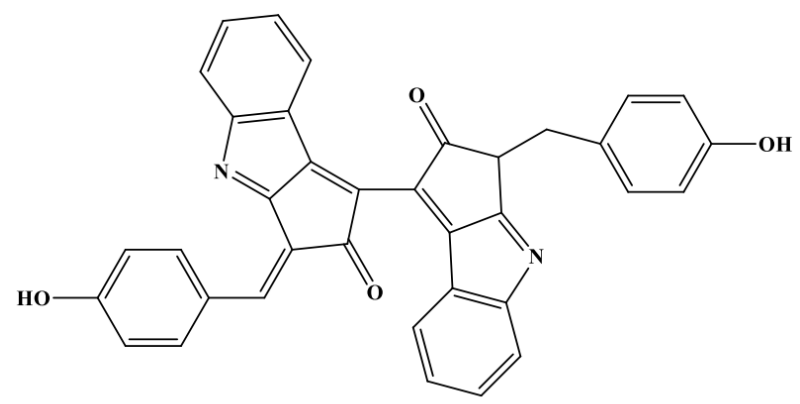

Scytonemin

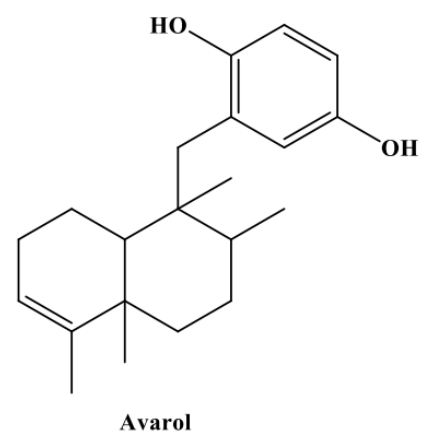

Avarol

Fig. 73: Chemical structures of some selected marine-derived compounds previously-mentioned in Table 1

Different products could find great interest in medical fields and were able to be produced and marketed globally. 

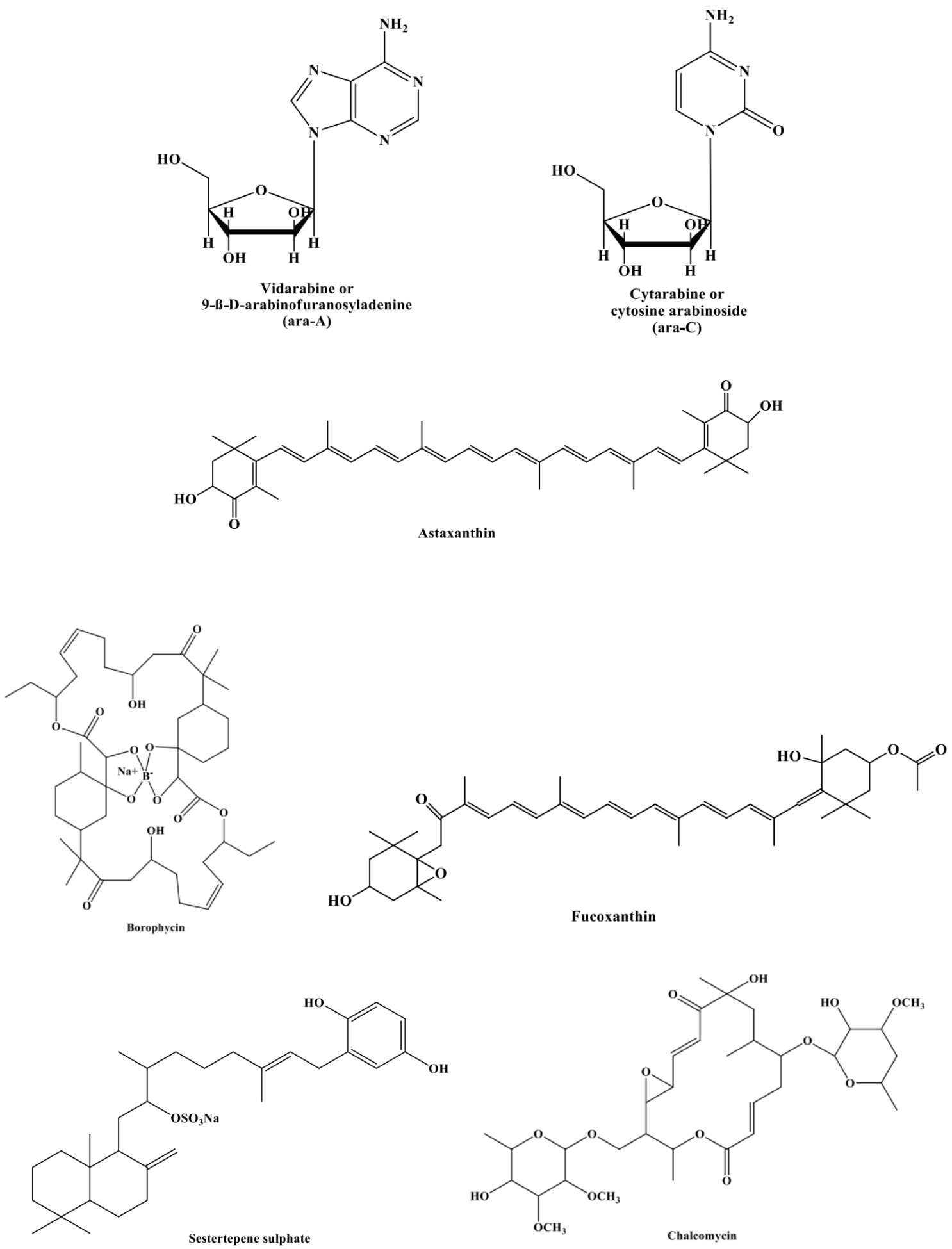

\section{Cont., Fig. 73: Chemical structures of some selected marine-derived compounds which were mentioned in Table 1. \\ Different products could find great interest in medical fields and were able to be produced and marketed globally.}




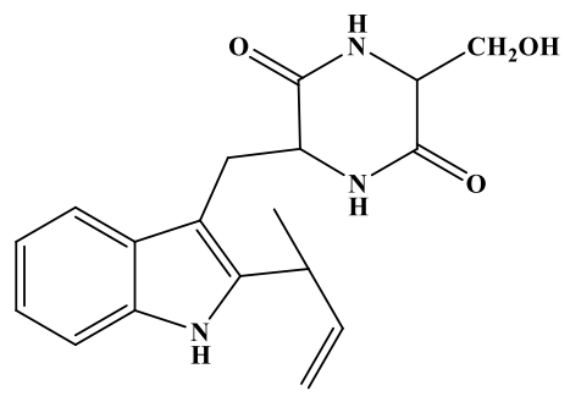

Cristatumins A

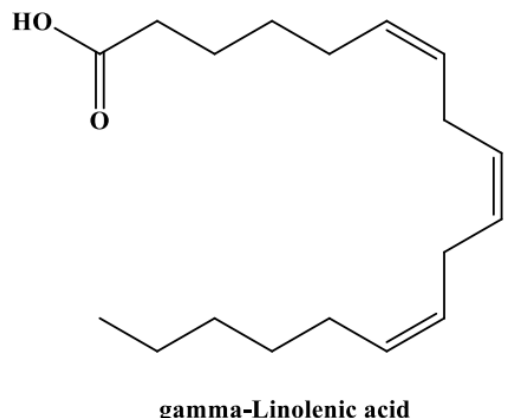<smiles>C=CC(C)c1[nH]c2ccccc2c1/C=C(\NC(=O)C(N)=O)C(=O)OC</smiles>

gamma-Linolenic acid

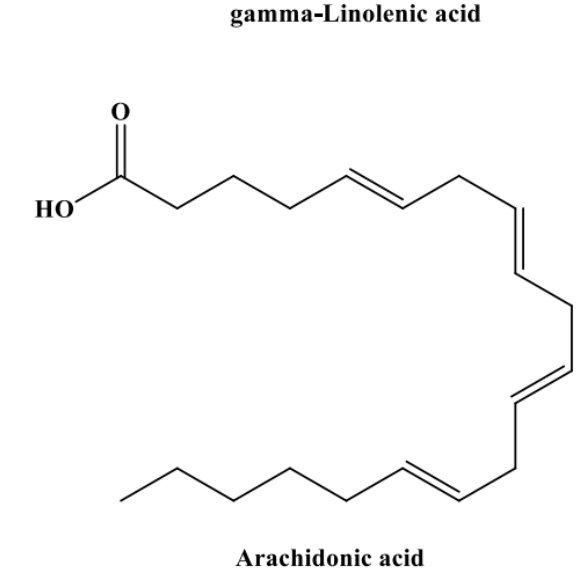

Arachidonic acid

Cristatumins D

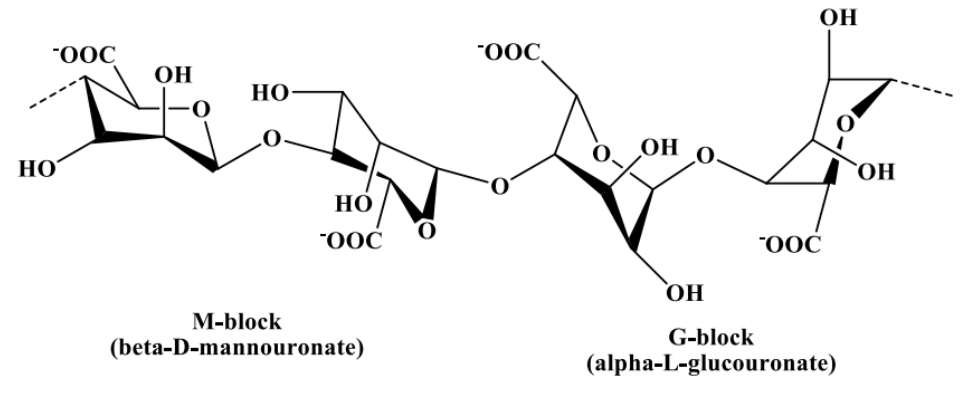

Alginate

Cont., Fig. 73: Chemical structures of some selected marine-derived compounds which were mentioned in Table 1.

Different products could find great interest in medical fields and were able to be produced and marketed globally. 

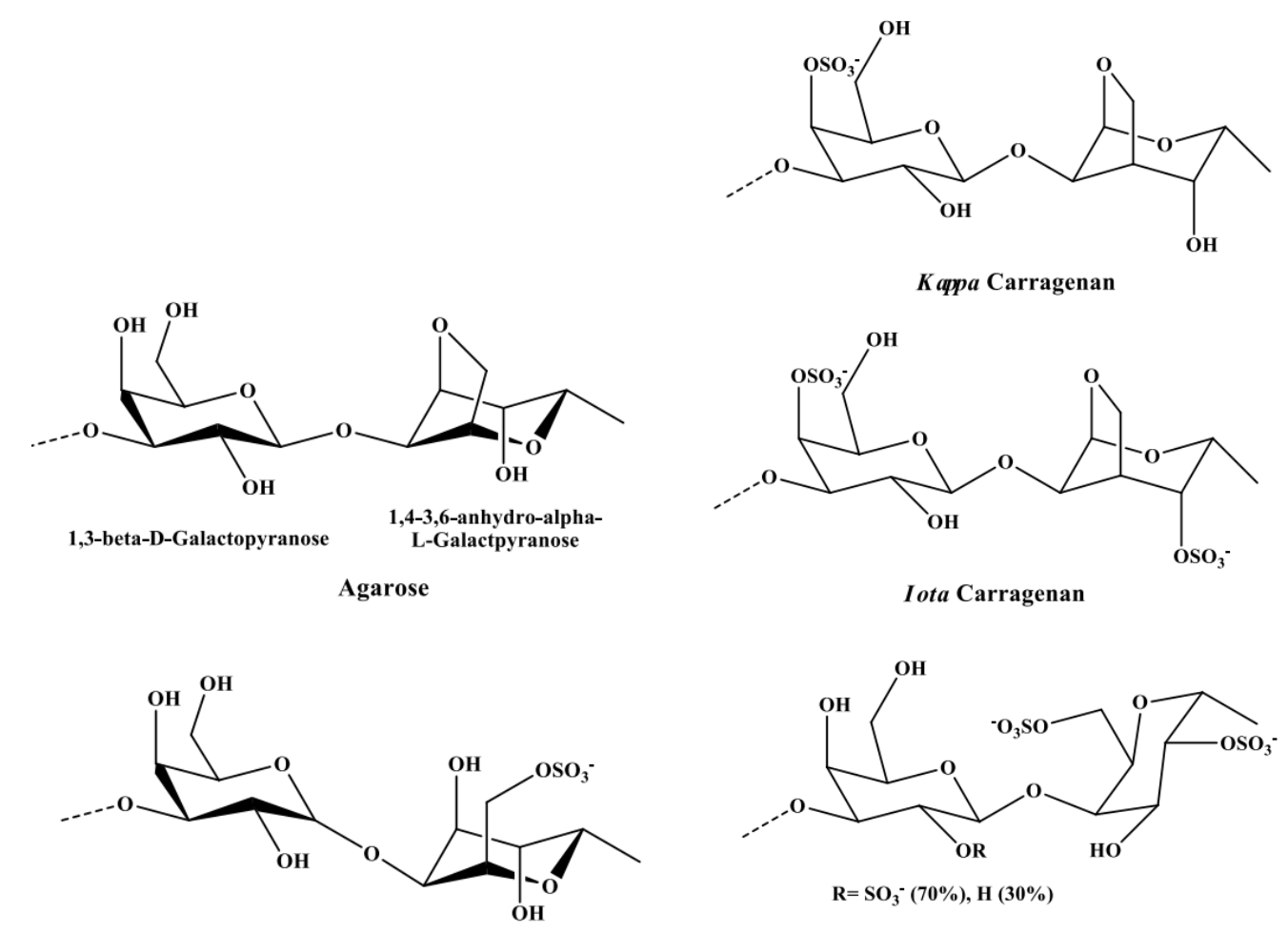

Agaropectin

Lambda Carragenan
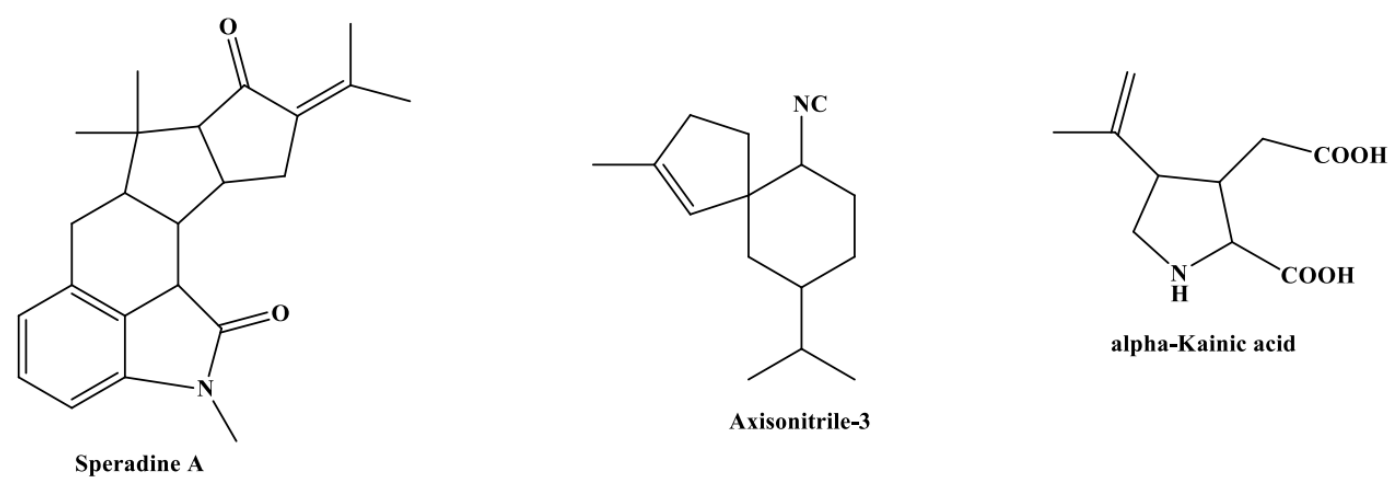

Axisonitrile-3

Cont., Fig. 73: Chemical structures of some selected marine-derived compounds which were mentioned in Table 1.

Different products could find great interest in medical fields and were able to be produced and marketed globally. 


\section{Appendix B}

\section{Reagents and buffers}

\section{1. $20 \mathrm{mM}$ Maleic acid buffer (MAB) (pH 1)}

\section{Component}

- Maleic acid

- Deionized water
Quantity

$2.32 \mathrm{~g}$

$900 \mathrm{~mL}$
Producer

TU Kaiserslautern

(Chemistry Department)

The solution was adjusted at $\mathrm{pH} 1$ before the volume had been completed to $1 \mathrm{~L}$.

\section{2. $\underline{0.06} \mathrm{mM}$ Toluidine blue $\mathrm{O}$}

\section{Component}

- Toluidine blue $\mathrm{O}$

- $\quad$ Maleic acid buffer (pH 1)

\section{Quantity}

$0.018 \mathrm{~g}$

to $1 \mathrm{~L}$
Producer

Sigma-Aldrich ${ }^{\circledR}$

3. $20 \mathrm{mM}$ 2-( $N$-morpholino) ethanesulfonic acid (MES) (pH 6 )

\section{Component}

- MES monohydrate

- Deionized water
Quantity

$2.32 \mathrm{~g}$

$900 \mathrm{~mL}$

The solution was adjusted at $\mathrm{pH} 6$ before the volume had been completed to $1 \mathrm{~L}$.

\section{Phosphate-buffered saline (1x) (PBS buffer) (pH 7.4)}

\section{Component}

- $\mathrm{Na}_{2} \mathrm{HPO}_{4} \cdot 2 \mathrm{H}_{2} \mathrm{O}$

- $\mathrm{NaHPO}_{4} \cdot \mathrm{H}_{2} \mathrm{O}$

- $\mathrm{KCl}$

- $\mathrm{NaCl}$

- Deionized water

$\begin{array}{cll}\text { Molarity (mM) } & \text { Quantity (g) } & \text { Producer } \\ 5.0 & 12.0 & \text { Sigma-Aldrich }^{\circledR} \\ 1.5 & 0.21 & \\ 2.7 & 0.2 & \text { Carl-Roth }^{\circledR} \\ 137 & 8.01 & \text { Sigma-Aldrich }^{\circledR} \\ & 900 \mathrm{~mL} & \end{array}$

The solution was adjusted at $\mathrm{pH} 7.4$ before the volume had been completed with deionized water to $1 \mathrm{~L}$. 
5. Toluidine blue, thionin acetate and perylene diimide derivative (as TFA salt) $\left(\mathrm{CF}_{3} \mathrm{CO}_{2} \underline{\mathrm{H}}\right)_{8}$ for immobilization

\begin{tabular}{|c|c|c|c|c|c|c|}
\hline Component & \multicolumn{3}{|c|}{ Toluidine blue $O$} & \multicolumn{2}{|c|}{ Thionin acetate } & Perylene diimide derivative \\
\hline Molarity (mM) & 2 & 4 & 6 & 2 & 4 & 2 \\
\hline Quantity (g) & 0.61 & 1.22 & 1.8 & 0.57 & 1.15 & $3.66 \mathrm{~g}$ \\
\hline Producer & \multicolumn{5}{|c|}{ Sigma-Aldrich $^{\circledR}$} & $\begin{array}{c}\text { University of Heidelberg } \\
\text { (Institute of inorganic } \\
\text { chemistry) }\end{array}$ \\
\hline
\end{tabular}

The different powders had been dissolved with $1 \mathrm{~L}$ deionized water.

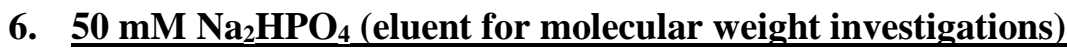

\begin{tabular}{|c|c|c|}
\hline Component & Quantity & Producer \\
\hline - $\mathrm{Na}_{2} \mathrm{HPO}_{4}$ & $7.1 \mathrm{~g}$ & Sigma-Aldrich $^{\circledR}$ \\
\hline - Deionized water & $1 \mathrm{~L}$ & \\
\hline
\end{tabular}

7. $\underline{0.9 \%(\mathrm{w} / \mathrm{v}) \mathrm{NaCl}}$

\section{Component}

$-\mathrm{NaCl}$

- $\quad$ Deionized water
Quantity

$9 \mathrm{~g}$

$1 \mathrm{~L}$
Producer

Sigma-Aldrich ${ }^{\circledR}$

\section{8. $100 \mathrm{mM} \mathrm{Na} \mathrm{S2O}_{4}$ in $20 \mathrm{mM}$ MES (pH 6)}

Component

- $\mathrm{Na}_{2} \mathrm{~S}_{2} \mathrm{O}_{4}$

- $\quad$ MES (pH 6)
Quantity

$17.4 \mathrm{~g}$

$1 \mathrm{~L}$
Producer

TU Kaiserslautern (Chemistry

Department) 


\section{Enzyme solution for protoplast isolation}

According to Mussio and Rusig [177], protoplast from F. vesiculosus was isolated by the following solution.

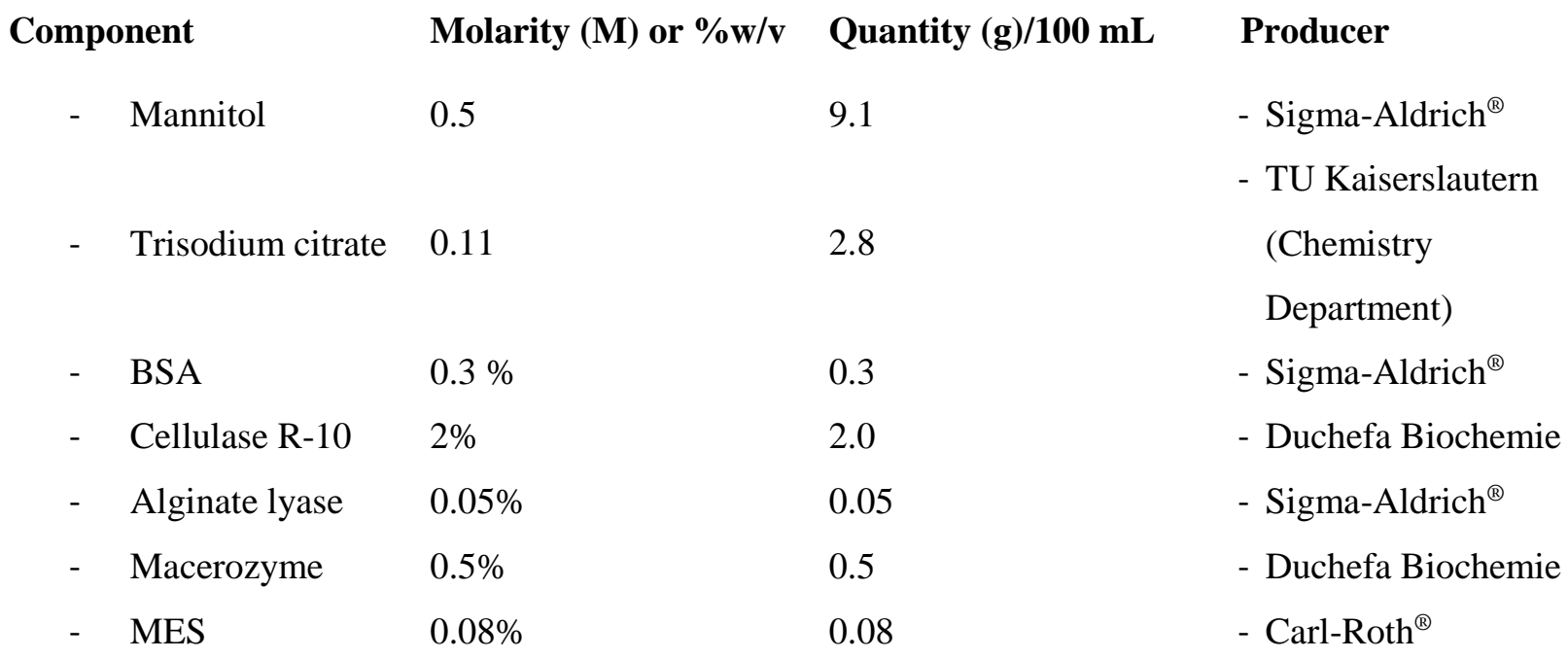

All constituents should be dissolved in autoclaved sea water and then filter sterilized and stored at $4{ }^{\circ} \mathrm{C}$.

\section{Protoplast washing solution}

\section{Component}

$-\mathrm{NaCl}$

- $\mathrm{CaCl}_{2} \cdot 2 \mathrm{H}_{2} \mathrm{O}$
Molarity (M)

0.4

0.005

\section{Quantity (g)/L Producer}

23.38

0.74

$\mathrm{pH}$ was adjusted at 7.0 before autoclaving.

\section{Antibiotic stock solutions (1000x) in LB cultivation medium}

\section{Antibiotic}

- Ampicillin sodium

- Kanamycin sulphate

- Chloramphenicol

\author{
Concentration (mg.mL $\left.\mathbf{m}^{-1}\right)$ \\ 100.0 in water \\ 50.0 in water \\ 34.0 in $70 \% \mathrm{v} / \mathrm{v}$ ethanol
}

\author{
Producer \\ AppliChem GmbH \\ Carl-Roth $^{\circledR}$ \\ AppliChem GmbH
}

Prepared stock antibiotic solutions should be sterile-filtered then stored at $-20{ }^{\circ} \mathrm{C}$.

Other antibiotics used in surface sterilization protocol were purchased from AppliChem GmbH. 
12. Agarose gel electrophoresis: 50x TAE buffer

\section{Component}

- $\quad$ Tris-acetate ( $\mathrm{pH} 8.5)$

- $\mathrm{Na}_{2}$ EDTA
Molarity (M)

2

0.05
Quantity (g)/L Producer

242.28

Carl-Roth $^{\circledR}$

18.61

13. Stock solutions in protein expression, detection and purification

13.1. General solutions

\section{Compound}

- Anhydrotetracycline (AHT)

- Ammonium Persulphate (APS)

- Avidin

- 5-Bromo-4-chloro-3-indolyl phosphate (BCIP)

- $\quad$ Nitro blue tetrazolium (NBT)

Prepared and stored at $-20{ }^{\circ} \mathrm{C}$.

13.2. SDS-PAGE and Western blot solutions

\subsubsection{0x SDS Running buffer}

\section{Component}

- $\quad$ Tris- $\mathrm{HCl}(\mathrm{pH} 8.3)$

- Glycine

- $\operatorname{SDS}(1 \% \mathrm{w} / \mathrm{v})$

13.2.2. $4 x$ Stacking gel buffer

\section{Component}

- $\quad$ Tris- $\mathrm{HCl}(\mathrm{pH} 6.8)$

- $\operatorname{SDS}(4 \% \mathrm{w} / \mathrm{v})$

\section{Molarity (M)}

0.25

1.92

0.035

\section{Concentration}

$2 \mathrm{mg} \cdot \mathrm{mL}^{-1}$ in DMF or ethanol

$10 \%(\mathrm{w} / \mathrm{v}) 1 \mathrm{~g} .10 \mathrm{~mL}^{-1}$ deionized water

$2 \mathrm{mg} \cdot \mathrm{mL}^{-1}$ deionized water

$50 \mathrm{mg} \cdot \mathrm{mL}^{-1}$ in DMF

$100 \mathrm{mg} \cdot \mathrm{mL}^{-1}$ in $70 \%(\mathrm{v} / \mathrm{v}) \mathrm{DMF}$

$\begin{array}{ll}\begin{array}{l}\text { Quantity }(\mathbf{g}) / \mathbf{L} \\ 30.28\end{array} & \begin{array}{l}\text { Producer } \\ \text { Carl-Roth }^{\circledR}\end{array} \\ 144.0 & \text { Sigma-Aldrich }^{\circledR} \\ 10.0 & \text { Carl-Roth }^{\circledR}\end{array}$

Quantity (g)/L Producer

Quantity (g)/200 mL

12.114

8.0 


\subsubsection{4x Separation gel buffer}

\section{Component}

- $\quad$ Tris- $\mathrm{HCl}(\mathrm{pH} 8.8)$

- $\quad \operatorname{SDS}(0.4 \% \mathrm{w} / \mathrm{v})$
Molarity (M)

1.5

0.0138
Quantity (g)/200 mL

36.34

0.8

\subsubsection{4x SDS sample buffer}

\section{Component}

- $\quad$ Tris- $\mathrm{HCl}(\mathrm{pH}$ 6.8)

- $\operatorname{SDS}(8 \% \mathrm{w} / \mathrm{v})$

- Glycerol

- $\quad \beta$-Mercaptoethanol

- Bromophenol blue

\section{Molarity (M)/\%w/v}

0.1

0.28

$40 \% \mathrm{v} / \mathrm{v}$

$10 \% \mathrm{v} / \mathrm{v}$

$0.015(1 \% \mathrm{w} / \mathrm{v})$

\section{Quantity (g)/10 mL Producer}

0.121

0.8

$4 \mathrm{~mL}$

$1 \mathrm{~mL}$

0.1

\subsubsection{SDS-Gel plate (12.5\%)}

In the beginning, the following reagents and gel should be prepared, as follows:

\begin{tabular}{|c|c|c|c|c|}
\hline \multicolumn{2}{|c|}{ Reagent } & Separation gel $(12.5 \%)$ & Stacking gel $(5.25 \%)$ & Stop gel \\
\hline- & Acrylamide $30 \%$ & $6.7 \mathrm{~mL}$ & $1.4 \mathrm{~mL}$ & $420 \mu \mathrm{L}$ \\
\hline- & Buffer & $4.0 \mathrm{~mL}$ & $2.0 \mathrm{~mL}$ & $250 \mu \mathrm{L}$ \\
\hline- & D.W & $5.3 \mathrm{~mL}$ & $4.6 \mathrm{~mL}$ & $330 \mu \mathrm{L}$ \\
\hline- & $10 \%$ APS & $80 \mu \mathrm{L}$ & $30 \mu \mathrm{L}$ & $13 \mu \mathrm{L}$ \\
\hline- & TEMED & $8 \mu \mathrm{L}$ & $20 \mu \mathrm{L}$ & $2 \mu \mathrm{L}$ \\
\hline
\end{tabular}

As shown in Fig. 74, each plate consists of $500 \mu \mathrm{L}$ of stop gel should be poured in between fitted glass plates in its casting chamber to form the basal guard layer of the gel. Afterwards, $3 \mathrm{~mL}$ of separation gel form the main part of gel then $1 \mathrm{~mL}$ of stacking buffer to be appeared as in the following figure. Prepared plates should be then stored in wet tissues at $4{ }^{\circ} \mathrm{C}$ till time of use. 


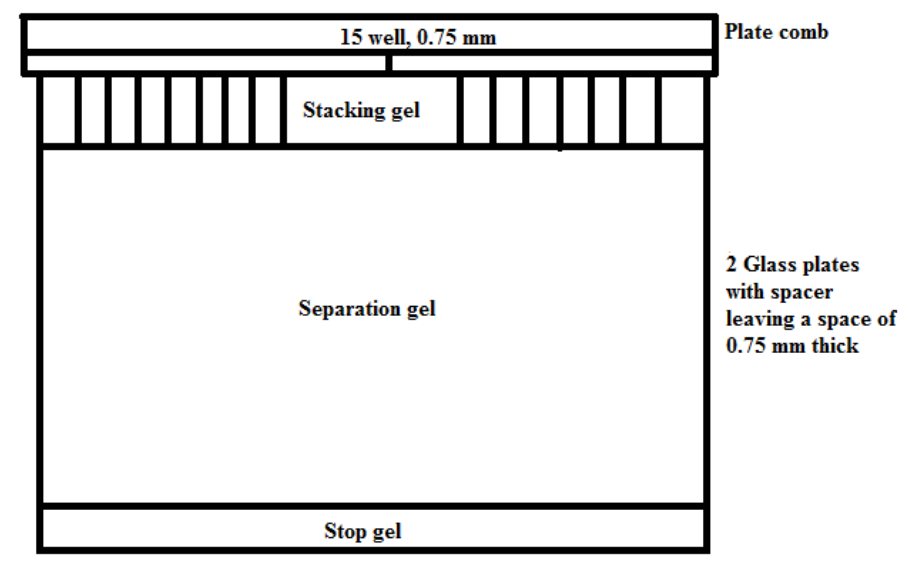

Fig. 74: SDS-PAGE plate showing its building components

\subsubsection{Coommassie blue staining solution}

\section{Component}

- $\quad$ Acetic acid (P. A., 99.8\%)

- $\quad$ Ethanol (Analytical grade, $\geq 99.8 \%$ ) 30

$\% \mathrm{v} / \mathrm{v} \quad$ Volume $\mathrm{mL} / \mathrm{L}$

$10 \quad 100$

- Coomassie Brilliant Blue G-250 $0.5 \%$ w $/ \mathrm{v} \quad 5.0 \mathrm{~g}$

Dye

\subsubsection{Coommassie blue bleaching solution}

\section{Component}

- $\quad$ Acetic acid (P. A., 99.8\%)

$\% \mathrm{v} / \mathrm{v}$

- $\quad$ Ethanol (Analytical grade, $\geq 99.8 \%$ ) 30

\subsubsection{Western Blot}

i. Towbin buffer

\section{Component}

- $\quad$ Tris (pH 8.3)

- Glycine
Molarity (M)

0.025

0.192
Volume mL/ L

100

300

$\mathrm{pH}$ should not be adjusted with acids or alkali.

Quantity (g)/L

3.028

14.4
Producer

TU Kaiserslautern

(Chemistry Department)

AppliChem GmbH 
ii. Alkaline phosphatase (AP buffer) $\mathrm{pH} 9.5$

\section{Component}

- $\quad$ Tris- $\mathrm{HCl}$

- $\mathrm{NaCl}$

- $\mathrm{MgCl}_{2}$
Molarity (M)

0.1

0.1

0.005
Quantity (g)/L Producer

3.028

Carl-Roth ${ }^{\circledR}$

5.85

1.02

Carl-Roth ${ }^{\circledR}$

iv. Blocking solution in PBS-T buffer

\section{Component}

- Albumin fraction V from BSA

- Complete volume to $100 \mathrm{~mL}$ by PBS-T

v. Purification buffer (Buffer W ( $\mathrm{pH}$ 8.0))

\section{Quantity (g)/ $100 \mathrm{~mL} \quad$ Producer}

3.0

\section{Component}

- $\quad$ Tris- $\mathrm{HCl}$

- $\quad \mathrm{NaCl}$
Molarity (M)

0.1

0.15
Quantity (g)/L

12.14

8.76

$1 \mathrm{mM}$ EDTA was excluded from IBA GmbH formula

vi. Elution buffer (Buffer E)

\section{Component}

- Desthiobiotin

- $\quad$ Buffer W was added to $1 \mathrm{~L}$
Molarity (M)

0.0025
Quantity (g)/L Producer

0.54 
vii. Regeneration buffer (Buffer R)

\section{Component}

- 2-[4-hydroxy-benzeneazo]benzoic acid 0.001 (HABA)

- Buffer W was added to $1 \mathrm{~L}$
Molarity (M) Quantity (g)/L

0.24

\section{Description of Sepabeads ${ }^{\circledR}$ EC-EA [98]}

$\begin{array}{ll}\text { Item } & \text { Description } \\ \text { Grade } & \text { S (small) } \\ \text { Particle size range } & 100-300 \mu \mathrm{m} \\ \text { Matrix } & \text { Polymethacrylate } \\ \text { Appearance } & \text { Perfect spherical white opaque beads } \\ \text { Functional group } & \text { Ethylenediamino } \\ \text { Functional group density } & \text { Min. } 600 \mu \text { mol/g wet } \\ \text { Median pore diameter } & 10-20 \mathrm{~nm} \\ \text { True density } & >1.1 \mathrm{~g} / \mathrm{mL} \\ \text { Water retention } & 55-65 \% \\ \text { Temperature stability range } & 2-60^{\circ} \mathrm{C} \\ \text { pH stability } & 1-14 \\ \text { Recommended storage temperature } & \text { Room temperature }\end{array}$




\section{Appendix C}

\section{Medium composition and preparations}

\section{Lysogeny Broth-Miller (LB)-agar medium [Tissue culture chapter]}

\begin{tabular}{|c|c|c|}
\hline Component & Quantity (g/L) & Producer \\
\hline - Tryptone & 10.0 & AppliChem GmbH \\
\hline - $\quad$ Yeast extract & 5.0 & \\
\hline - $\quad \mathrm{NaCl}$ & 10.0 & \\
\hline - Agar & 15.0 & Carl-Roth $^{\circledR}$ \\
\hline
\end{tabular}

Medium components (without agar) had been dissolved in deionized water ( $\left.\mathrm{pH} 6.8-7.0,24.0{ }^{\circ} \mathrm{C}\right)$, then it was autoclaved after the addition of agar.

\section{Marine Boullion (MB50)-Agar medium}

\begin{tabular}{|c|c|c|}
\hline Component & Quantity (g/L) & Producer \\
\hline - $\quad$ Marine Broth $\left(\right.$ Carl Roth $\left.{ }^{\circledR}\right)$ & 20.0 & Carl-Roth $^{\circledR}$ \\
\hline$-\quad \mathrm{NaCl}$ & 9.7 & \\
\hline - $\quad \operatorname{Agar}(1.5 \% \mathrm{w} / \mathrm{v})$ & 15.0 & \\
\hline
\end{tabular}

Medium components (without agar) had been dissolved in deionized water $\left(\mathrm{pH} 6.8-7.0,24.0{ }^{\circ} \mathrm{C}\right)$, then it was autoclaved after the addition of agar.

\section{Murashige and Skoog (MS) medium (pH 5.8) supplemented with $3 \%$ (w/v) sucrose}

\begin{tabular}{|c|c|c|}
\hline Component & Quantity (g/L) & Producer \\
\hline - Sucrose & 30.0 & Carl-Roth $^{\circledR}$ \\
\hline$-\quad$ MS & 4.4 & AppliChem GmbH \\
\hline _ Agar & 5.5 & Carl-Roth $^{\circledR}$ \\
\hline
\end{tabular}

4. Nutrient agar medium for cultivation of Rhizopium radiobacter DSM30147

(as described by Leibniz Institute DSMZ-German Collection of Microorganisms and Cell Cultures)

\begin{tabular}{|c|c|c|}
\hline Component & Quantity (g/L) & Producer \\
\hline - Pepton (Soya) & 5.0 & Sigma-Aldrich $^{\circledR}$ \\
\hline Meat extract & 3.0 & AppliChem GmbH \\
\hline - $\quad$ Agar (for solid medium) & 15.0 & Carl-Roth $^{\circledR}$ \\
\hline
\end{tabular}

Constituents were dissolved in $900 \mathrm{~mL}$ deionized water and $\mathrm{pH}$ was adjusted to 7.0, before the addition of agar. Volume was then completed to $1 \mathrm{~L}$ before autoclaving. 


\section{LB Broth medium (Liquid and solid media) [Lennox][for heterologous expression chapter]}

\section{Component}

- Tryptone

- $\quad$ Yeast extract

$-\mathrm{NaCl}$

- Agar (for solid medium)
Quantity (g/L)

10.0

5.0

5.0

15.0
Producer

Carl-Roth ${ }^{\circledR}$

Complete volume to 1000

Prepared medium was also purchased from Carl-Roth ${ }^{\circledR}$. In this case, $20 \mathrm{~g}$ of the medium powder was dissolved in $1 \mathrm{~L}$ deionized water. 1000x Sterile-filtered antibiotic solution $(1 \mathrm{~mL})$ should be added to a cooled autoclaved medium.

\section{PES medium}

In a ratio of 1:50, $20 \mathrm{~mL}$ of Enrichment Stock Solution (ESS) was diluted to $1 \mathrm{~L}$ using $0.45 \mu \mathrm{m}$ filtered seawater to produce PES medium. $\mathrm{pH}$ should be adjusted at 7.8 before autoclaving. In addition, vitamins should be added to cooled autoclaved medium. In washing steps, an alternative medium was used (PESA), in which PES medium was used without vitamins.

\subsection{Enrichment Stock Solution (ESS) (50x)}

\section{Component}

- $\quad$ Tris base

- $\mathrm{NaNO}_{3}$

- $\quad \mathrm{Na}_{2} \beta$-glycerophosphate. $\mathrm{H}_{2} \mathrm{O}$

- Iron-EDTA solution (see 14.2)

- Trace metal solution (see below)

- Vitamin B1 (Thiamine.HCl)

- Vitamin H (Biotin)

- Vitamin B12 (Cyanocobalamin)
Quantity (g) /L

5.0

3.5

0.5

$250 \mathrm{~mL}$

$25 \mathrm{~mL}$

$5 \times 10^{-4}$

$5 \times 10^{-6}$

$1 \times 10^{-5}$
Producer

Carl-Roth $^{\circledR}$

Merck

AppliChem GmbH

Carl-Roth $^{\circledR}$

Sigma-Aldrich ${ }^{\circledR}$

Carl-Roth $^{\circledR}$

\subsection{Iron-EDTA solution}

\section{Component}

- $\mathrm{Na}_{2}$ EDTA. $2 \mathrm{H}_{2} \mathrm{O}$

- $\mathrm{Fe}\left(\mathrm{NH}_{4}\right)_{2}\left(\mathrm{SO}_{4}\right)_{2} \cdot 6 \mathrm{H}_{2} \mathrm{O}$
Quantity (g) /L

6.0

7.0
Producer

Carl-Roth $^{\circledR}$

Merck 


\subsection{Trace metal solution}

\section{Component}

- $\mathrm{Na}_{2}$ EDTA. $2 \mathrm{H}_{2} \mathrm{O}$

- $\mathrm{H}_{3} \mathrm{BO}_{3}$

- $\mathrm{MgSO}_{4} \cdot 7 \mathrm{H}_{2} \mathrm{O}$

- $\mathrm{ZnSO}_{4} .7 \mathrm{H}_{2} \mathrm{O}$

- $\mathrm{CoSO}_{4} \cdot 7 \mathrm{H}_{2} \mathrm{O}$
Quantity (g/L)

2.54

2.24

0.24

0.044

0.01
Producer

Carl-Roth $^{\circledR}$

Merck

Merck

TU Kaiserslauern

(Chemistry

Department)

\section{ASP-12-NTA medium}

Medium constituents were completely dissolved in deionized water with aid of ultrasound. The medium $\mathrm{pH}$ was adjusted to 7.8 before autoclaved $\left(20 \mathrm{~min}, 121^{\circ} \mathrm{C}, 2 \mathrm{bar}\right)$. Sterile-filtered vitamin solutions were added to a room-temperature autoclaved medium.

\section{Component}

$-\mathrm{NaCl}$

- $\mathrm{KCl}$

- $\mathrm{MgSO}_{4} .7 \mathrm{H}_{2} \mathrm{O}$

- $\mathrm{MgCl}_{2} \cdot 6 \mathrm{H}_{2} \mathrm{O}$

- $\mathrm{CaCl}_{2} \cdot 2 \mathrm{H}_{2} \mathrm{O}$

- $\mathrm{NaNO}_{3}$

- $\mathrm{K}_{3} \mathrm{PO}_{4}$

- $\quad \mathrm{Na}_{2} \beta$-glycerophosphate. $\mathrm{H}_{2} \mathrm{O}$

- $\mathrm{Na}_{2} \mathrm{SiO}_{3} .9 \mathrm{H}_{2} \mathrm{O}$

- PII metal solution

- SII metal solution

- Nitrilotriacetic acid

- Tris

- Vitamin B1 (Thiamine.HCl)

- Vitamin H (Biotin)

- Vitamin B12 (Cyanocobalamin)

\section{Quantity (g) /L} 20

0.7

7.0

4.0

0.4

0.1

0.01

0.01

0.15

$10 \mathrm{~mL}$

$10 \mathrm{~mL}$

0.1

1.0

0.1

$1 \times 10^{-3}$

$2 \times 10^{-4}$

\section{Producer}

Sigma-Aldrich ${ }^{\circledR}$

Sigma-Aldrich ${ }^{\circledR}$

Merck

Sigma-Aldrich $^{\circledR}$

Sigma-Aldrich ${ }^{\circledR}$

Merck

Sigma-Aldrich ${ }^{\circledR}$

AppliChem GmbH

Sigma-Aldrich ${ }^{\circledR}$

TU Kaiserslauern

(Chemistry Department)

Carl-Roth $^{\circledR}$

Carl-Roth $^{\circledR}$

Sigma-Aldrich ${ }^{\circledR}$

Carl-Roth $^{\circledR}$ 


\subsection{PII metal solution}

\section{Component}

- $\mathrm{Na}_{2}$ EDTA. $2 \mathrm{H}_{2} \mathrm{O}$

- $\mathrm{FeCl}_{3}$

- $\mathrm{H}_{3} \mathrm{BO}_{3}$

- $\mathrm{MgCl}_{2} \cdot 2 \mathrm{H}_{2} \mathrm{O}$

- $\mathrm{ZnCl}_{2} \cdot 7 \mathrm{H}_{2} \mathrm{O}$

- $\mathrm{CoCl}_{2}$

\subsection{SII metal solution}

\section{Component}

- $\mathrm{NaBr}$

- $\mathrm{SrCl}_{2}$

- $\mathrm{RbCl}$

- $\mathrm{LiCl}$

- $\mathrm{Na}_{2} \mathrm{MoO}_{4}$

- $\mathrm{KI}$
Quantity (g) /100 mL

0.1

0.02

0.002

0.002

0.005

0.0001
Quantity (g) /100 mL

0.1

0.001

0.02

0.004

0.0005

0.0001
Producer

Carl-Roth $^{\circledR}$

TU Kaiserslauern

(Chemistry Department)

Meck

Carl-Roth $^{\circledR}$

TU Kaiserslauern

(Chemistry Department)

TU Kaiserslauern

(Chemistry Department)

\section{Producer}

TU Kaiserslauern

(Chemistry Department)

Sigma-Aldrich ${ }^{\circledR}$

Sigma-Aldrich ${ }^{\circledR}$

TU Kaiserslauern

(Chemistry Department)

Sigma-Aldrich $^{\circledR}$

TU Kaiserslauern

(Chemistry Department)

\section{F/2 medium}

A sterile-filtered 50x Guillard's (F/2) Marine Enrichment medium was purchased from Sigma-Aldrich ${ }^{\circledR}$. For the production of a $1 \mathrm{~L}$ medium, $20 \mathrm{~mL}$ of medium was diluted by $980 \mathrm{~mL} 0.45 \mu \mathrm{m}$ filtered and autoclaved sea water. 
9. Protoplast cultivation medium [177]

\section{Component}

- HEPES

- $\quad$ PESA (3.8\% sea salt)

- Glucose

- Sucrose

- Casein hydrolysate

- Ornithine hydrochloride

- $\quad$ CPPU

$-\quad 2,4-\mathrm{D}$
Molarity / \%w/v

$2 \mathrm{mM}$

$1 \% \mathrm{v} / \mathrm{v}$

$250 \mathrm{mM}$

$150 \mathrm{mM}$

$0.025 \%$

$20 \mu \mathrm{M}$

$0.4 \mu \mathrm{M}$

$0.45 \mu \mathrm{M}$
Quantity (g) Producer $/ 100 \mathrm{~mL}$

0.047

$1.0 \mathrm{~mL}$

4.5

5.1

0.025

$3.3 \times 10^{-4}$

$9.9 \times 10^{-3}$

$1 \times 10^{-5}$
Carl-Roth $^{\circledR}$

Sigma-Aldrich ${ }^{\circledR}$

Carl-Roth ${ }^{\circledR}$

Sigma-Aldrich ${ }^{\circledR}$

Duchefa

Biochemie

Duchefa

Biochemie

Duchefa

Biochemie 


\section{Appendix D}

\section{Protocols}

\section{Heat shock bacterial transformation protocol}

i. E. coli JM83 and BL21 (DE3) competent cells were provided from Prof. Dr. Nicole FrankenbergDinkel's lab. (Institute of Microbiology, TU Kaiserslautern) in a $1.5 \mathrm{~mL}$ reaction vessel as $0.5 \mathrm{~mL}$ samples. The cells were preserved in a cryopreservation refrigerator Cells were thawed on ice, firstly for 5 min. $1 \mu \mathrm{L}$ of $100 \mathrm{ng} \mu \mathrm{L}^{-1}$ dissolved DNA constructs was added afterwards.

ii. Cells were incubated with constructs on ice for $30 \mathrm{~min}$.

iii. The mixtures were then heat shocked at $42{ }^{\circ} \mathrm{C}$ for $120 \mathrm{~s}$.

iv. $700 \mu \mathrm{L}$ of $\mathrm{LB}$ broth was added afterwards to the tube and the transformation reactions placed in a shaker incubator at $37^{\circ} \mathrm{C}$ for $45-60 \mathrm{~min}$.

v. At the end of this incubation, centrifugation was carried out for $1 \mathrm{~min}$. at $13200 \mathrm{rpm}$ and/or spread 100 $\mu \mathrm{L}$ of the cell suspension on LB agar plates supplemented with the suitable antibiotic.

vi. Finally, plates were incubated overnight for approx. $16 \mathrm{~h}$ at $37{ }^{\circ} \mathrm{C}$ to get colonies of genetically transformed E. coli with corresponding required DNA.

\section{PCR protocol (Thermo Fisher Scientific ${ }^{\circledR}$ )}

Each reaction tube should be incubated in ice, while constituents were added:

- $\quad 25 \mu \mathrm{L}$ Dream-Tag ${ }^{\mathrm{TM}}$ green PCR Master Mix (2x),

- $\quad 2 \mu \mathrm{L}$ fwd primer $(20 \mu \mathrm{M})$,

- $\quad 2 \mu \mathrm{L}$ rev primer $(20 \mu \mathrm{M})$,

- $\quad 1 \mu \mathrm{L}$ DNA template (50 ng), and

- $\quad 20 \mu \mathrm{L}$ sterile highly pure water.

The PCR reaction afterwards was performed, according Table 18.

Table 18: PCR steps used in amplification of synthetic Esi0021_0026, Esi0050_0098, Esi0283_0018 and Esi0032_0064

\begin{tabular}{|c|c|c|c|}
\hline Step & Temperature $\left({ }^{\circ} \mathbf{C}\right)$ & Time & No. of cycles \\
\hline Initial denaturation & 95 & $3 \mathrm{~min}$ & 1 \\
\hline Denaturation & 95 & $30 \mathrm{~s}$ & 30 \\
\hline Annealing & Depend on primer's Tm & $30 \mathrm{~s}$ & \\
\hline Extension & 72 & $1 \mathrm{~min}$ & \\
\hline Final extension & 72 & $10 \mathrm{~min}$ & 1 \\
\hline Storage & 4 & \multicolumn{2}{|c|}{ forever } \\
\hline
\end{tabular}

3. Gibson assembly transformation protocol, according to Gibson assembly cloning kit instruction manual [254]

i. Thaw chemically competent cells on ice.

ii. Add $2 \mu \mathrm{L}$ of the chilled assembly product to the competent cells. Mix gently by pipetting up and down for 4-5 times. Do not vortex.

iii. Place the mixture on ice for $30 \mathrm{~min}$. Do not mix.

iv. Heat shock at $42{ }^{\circ} \mathrm{C}$ for $30 \mathrm{~s}$. Do not mix. 
v. Transfer tubes to ice for $2 \mathrm{~min}$.

vi. $\quad$ Add $950 \mu \mathrm{L}$ of room-temperature SOC media to the tube.

vii. Incubate the tube at $37^{\circ} \mathrm{C}$ for $60 \mathrm{~min}$. Shake vigorously $(250 \mathrm{rpm})$ or rotate.

viii. Spread $100 \mu 1$ of the cells onto the selection plates.

ix. Incubate overnight at $37^{\circ} \mathrm{C}$.

\section{Chromogenic detection of Strep-tag ${ }^{\circledR}$ fusion proteins with alkaline phosphatase (Western blot)}

According to the protocol described by IBA BioTAGnology [258] with some modifications, steps of protocols were performed as follow:

i. After SDS-PAGE, electro-transfer of proteins via Semi Dry-Blot technique to a PVDF membrane (previously activated by incubation with $\mathrm{MeOH}$ for $5 \mathrm{~min}$ ) was performed firstly. Membrane and Whatman filter papers were equilibrated with Towbin buffer, before protein transfer, which is carried out for $20 \mathrm{~min}$ at $15 \mathrm{~V}$ using an electrotansfer cell.

ii. Block the membrane with $20 \mathrm{ml}$ PBS-blocking buffer (3\% w/v albumin Fraction V in PBS-T buffer) by incubation for $1 \mathrm{~h}$ (room temperature; with gentle shaking) or overnight $\left(4^{\circ} \mathrm{C}\right)$.

iii. Wash three times with $20 \mathrm{ml}$ PBS-T buffer (each step: 5 min., room temperature, gentle shaking).

iv. After the last washing step, add $10 \mathrm{ml} 2 \mu \mathrm{g} \cdot \mathrm{mL}^{-1}$ avidine in PBS-T buffer to the membrane (10 minutes, room temperature, gentle shaking). This blocks endogenously biotinylated proteins (e.g. the biotin carboxyl carrier protein (BCCP, $22 \mathrm{kDa}$ ) in case of E. coli) which will otherwise stain sensitively.

v. Add $2.5 \mu \mathrm{L}$ Strep-Tactin ${ }^{\circledR}$ labeled with alkaline phosphatase conjugate to $10 \mathrm{~mL}$ PBS-T buffer (1:4000). Incubate $60 \mathrm{~min}$. at room temperature with gentle shaking.

vi. Wash three times with PBS-T buffer (each step: $5 \mathrm{~min}$, room temperature, gentle shaking).

vii. Wash three times with PBS-buffer (each step: $5 \mathrm{~min}$, room temperature, gentle shaking).

viii. Equilibrate the membrane with $10 \mathrm{~mL}$ alkaline phosphatase buffer for 5 min with gentle shaking. Add $33 \mu \mathrm{L}$ nitrotetrazolium blue (NBT; $100 \mathrm{mg} \cdot \mathrm{mL}^{-1}$ ) and $66 \mu \mathrm{L}$ 5-bromo-4-chloro-3-indolyl-phosphate (BCIP; $50 \mathrm{mg} \mathrm{mL}^{-1}$ ) to a $10 \mathrm{~mL}$ fresh alkaline phosphatase buffer.

ix. Proceed the chromogenic reaction under shaking until optimal signal:background ratio is achieved.

x. Stop reaction by washing several times with deionized water.

xi. Air dry the membrane and store it in the dark. 


\section{Production of adsorbent (immobilized TB, TA and PDD) [93]}

\begin{tabular}{|c|c|c|c|}
\hline Step 1 & Derivatization of the adsorbent & $\begin{array}{l}\text { i. } \\
\text { ii. } \\
\text { iii. } \\
\text { i. }\end{array}$ & $\begin{array}{l}500 \mathrm{mg} \text { Sepabeads }{ }^{\circledR} \text { EC-EA } \\
0.5 \mathrm{~mL} 50 \% \text { Glutardialdehyd solution (Glutaraldehyd=1,5-Pentandial) } \\
4.5 \mathrm{Ml} 20 \mathrm{mM} \text { MES (pH 6) } \\
24 \mathrm{~h} \text { incubation in an overhead shaker (F1 mode, } 30 \mathrm{rpm})\end{array}$ \\
\hline Step 2 & Washing & $\begin{array}{l}\text { ii. } \\
\text { iii. }\end{array}$ & $\begin{array}{l}\text { Three times with } 6.6 \mathrm{~mL} \mathrm{H}_{2} \mathrm{O} \\
24 \mathrm{~h} \text { incubation in an overhead shaker ( } \mathrm{F} 1 \text { mode, } 30 \mathrm{rpm})\end{array}$ \\
\hline Step 3 & $\begin{array}{l}\text { Simultaneous reduction with } \\
\mathrm{Na}_{2} \mathrm{~S}_{2} \mathrm{O}_{4} \text { and dyes immobilization }\end{array}$ & i. & $\begin{array}{l}\text { Add } 6.6 \mathrm{~mL} 100 \mathrm{mM} \mathrm{Na}_{2} \mathrm{~S}_{2} \mathrm{O}_{4} \text { in } 20 \mathrm{mM} \text { MES (pH6), } 0.5 \mathrm{~mL} 2 \mathrm{mM} \text { aqueous TB, TA } \\
\text { or PDD solutions } \\
12 \mathrm{~h} \text { incubation in an overhead shaker ( } \mathrm{F} 1 \text { mode, } 30 \mathrm{rpm})\end{array}$ \\
\hline Step 4 & Washing & $\begin{array}{l}\text { i. } \\
\text { ii. }\end{array}$ & $\begin{array}{l}\text { Three times } 6.6 \mathrm{~mL} \mathrm{H} \mathrm{H}_{2} \mathrm{O} \\
24 \mathrm{~h} \text { incubation in an overhead shaker ( } \mathrm{F} 1 \text { mode, } 30 \mathrm{rpm})\end{array}$ \\
\hline Step 5 & & $\begin{array}{l}\text { iii. } \\
\text { iv. }\end{array}$ & $\begin{array}{l}\text { Three times with } 6.6 \mathrm{~mL} 5 \mathrm{M} \mathrm{NaCl} \text { in } 20 \mathrm{mM} \mathrm{MAB}, \mathrm{pH} 1 \\
24 \mathrm{~h} \text { incubation in an overhead shaker (F1 mode, } 30 \mathrm{rpm})\end{array}$ \\
\hline Step 6 & & $\begin{array}{l}\text { v. } \\
\text { vi. }\end{array}$ & $\begin{array}{l}\text { Three times with } 6.6 \mathrm{~mL} \mathrm{H}_{2} \mathrm{O} \\
24 \mathrm{~h} \text { incubation in an overhead shaker ( } \mathrm{F} 1 \text { mode, } 30 \mathrm{rpm})\end{array}$ \\
\hline Step 7 & Drying & i. & Dry at $50^{\circ} \mathrm{C}$ until obtaining dried cyan or red-coloured beads \\
\hline
\end{tabular}




\section{Application of particles for the purification of fucoidan from crude extract [93]}

Step 1 A stock solution of crude extract with $2.5 \mathrm{mg} \mathrm{mL}^{-1}$ fucoidan in $20 \mathrm{mM}$ MAB (pH 1) or $20 \mathrm{mM}$ MES (pH 6) was prepared

Step 2 - Adsorption phase

Step 3 - Washing phase

Step 4

Step 5 - Elution phase

Step 6 Washing and storage

Step 7

Step 8

Step 9
- Removal of ethanol and concentration of eluate

- Dialysis and removal of $\mathrm{NaCl}$

- Lyophilization of dialyzed eluate
- $\quad$ To 50 or $75 \mathrm{mg}$ derivatized beads (adsorbents) add $1.5 \mathrm{~mL} 2.5 \mathrm{mg} \mathrm{mL}^{-1}$ crude extract Incubation for $44 \mathrm{~h}$ room temperature for thiazine dyes or $16 \mathrm{~h}$ for perylene diimide derivatized beads ( $\mathrm{F} 1$ mode, $30 \mathrm{rpm}$ )

- Centrifuge and remove the supernatant to be analyzed (calculation of adsorption rate and $\%$ of adsorbed fucoidan)

- Washing the adsorbents for $3 \mathrm{~h}$ with $1 \mathrm{~mL} \mathrm{H}_{2} \mathrm{O}$ at room temperature.

- Centrifuge and take a sample from supernatant for analysis

- Washing with $1 \mathrm{~mL}$ of $0.1 \mathrm{M} \mathrm{NaCl}$ prepared in $20 \mathrm{mM} \mathrm{MAB}(\mathrm{pH}$ 2) for $5 \mathrm{~h}$ at room temperature ( $\mathrm{F} 1$ mode, $30 \mathrm{rpm})$

- Centrifuge and remove the supernatant.

- Incubation of $1.5 \mathrm{~mL} 3 \mathrm{M} \mathrm{NaCl}$ prepared in $30 \%(\mathrm{v} / \mathrm{v})$ ethanol with loaded derivatized beads for $16 \mathrm{~h}$ in a thermoshaker $\left(50^{\circ} \mathrm{C}, 800 \mathrm{rpm}\right)$.

- Centrifuge and collect the eluate

- Washing adsorbents with $1 \mathrm{~mL} \mathrm{20 \%} \mathrm{ethanol} \mathrm{in} 20 \mathrm{Mm}$ MES pH 6 for $8 \mathrm{~h}$ (room temperature, $30 \mathrm{rpm}$ )

- Storage of the adsorbents in $20 \%$ ethanol in $20 \mathrm{Mm}$ MES pH 6

- In a rotary evaporator $\left(60{ }^{\circ} \mathrm{C}, 100 \mathrm{mbar}\right)$ eluate was concentrated (1:0.3)

- In a Dialysis membrane of MWCO (3.5 kDa) concentrated eluate was dialyzed against completely deionized water

- The process should be stopped when conductivity of replaced water (every 2-3 h) had stable and similar to fresh deionized water for at least $2 \mathrm{~h}$.

- In a lyophilizer $\left(-20^{\circ} \mathrm{C}, 1.03 \mathrm{mbar}\right)$, frozen eluate was transformed to fluffy powdered fucoidan. 


\section{Appendix E}

Calibration curves, instruments' charts and analysis reports

\section{Calibration curves}

1.1. Total sugar content in crude fucoidan

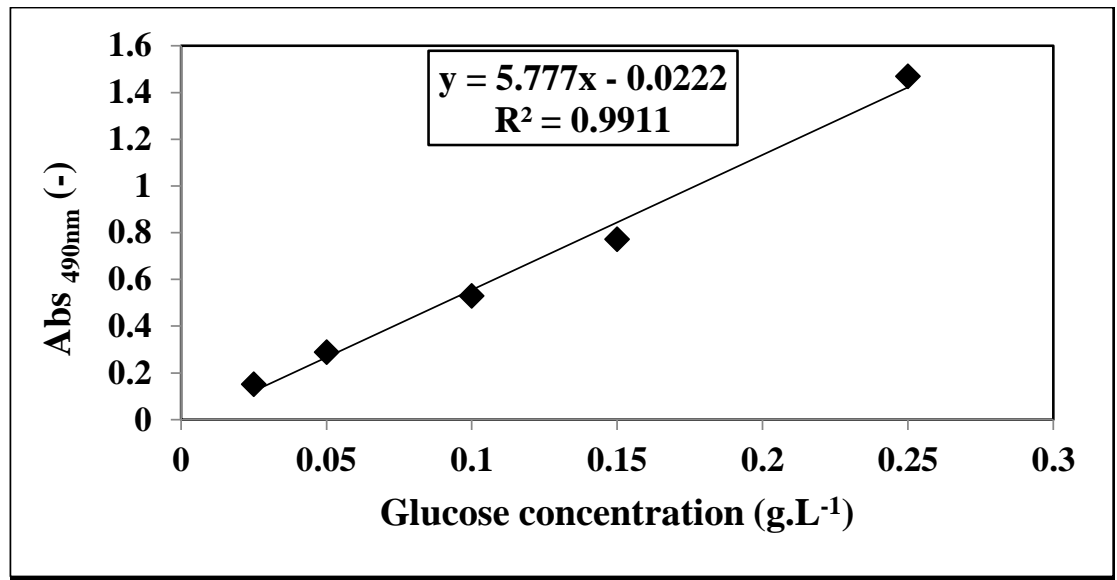

Fig. 75: Calibration curve of Dubois or phenol-sulphuric acid assay

Glucose was used as a standard and absorbance was measured at $490 \mathrm{~nm}$.

1.2. Fucoidan content and purity of crude fucoidan

a. TB assay

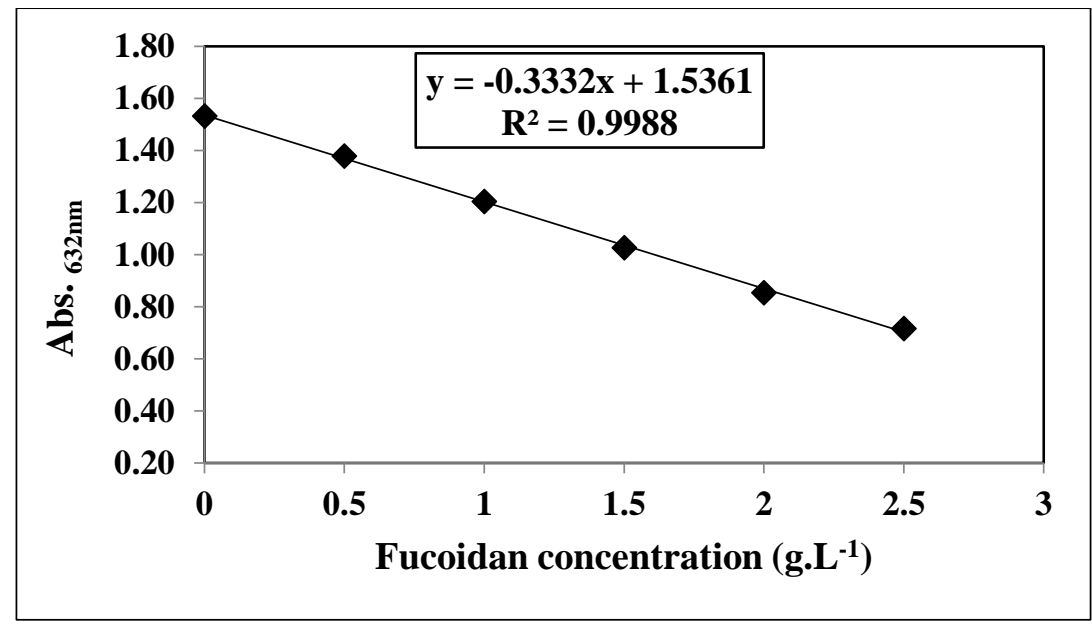

Fig. 76: Calibration curve of TB assay

Fucoidan $\left(>95 \%\right.$ pure, Sigma-Aldrich $\left.{ }^{\circledR}\right)$ was used as a standard at different serial dilution and reacted with0.06 mM TB and absorbance was measured at $632 \mathrm{~nm}$. 
b. Heparin $\operatorname{Red}^{\circledR}$ assay

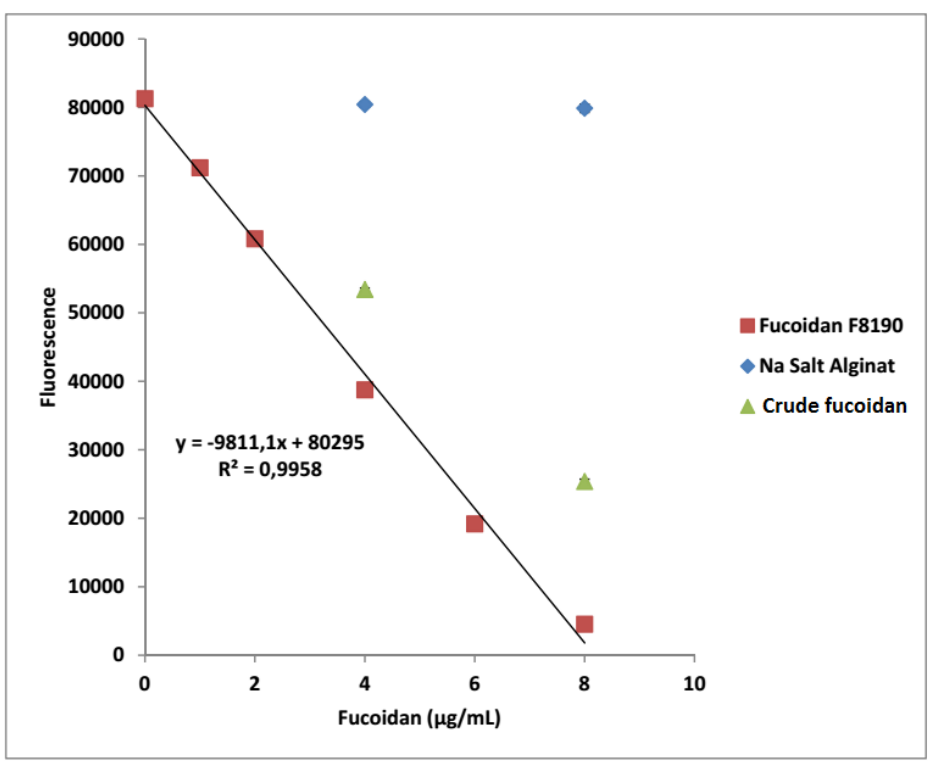

Fig. 77: Calibration curve of Heparin $\operatorname{Red}^{\circledR}$ Ultra assay

The figure shows replicates from crude fucoidan (Fucoidan_A) and alginate sodium salts to confirm that there selectivity of the assay to fucoidan. Fucoidan (F8190, >95\% pure SigmaAldrich $^{\circledR}$ ) was used as a standard. Excitation $\lambda$ at $570 \mathrm{~nm}$ and emission was recorded at $605 \mathrm{~nm}$.

1.3. Fucose content in crude fucoidan

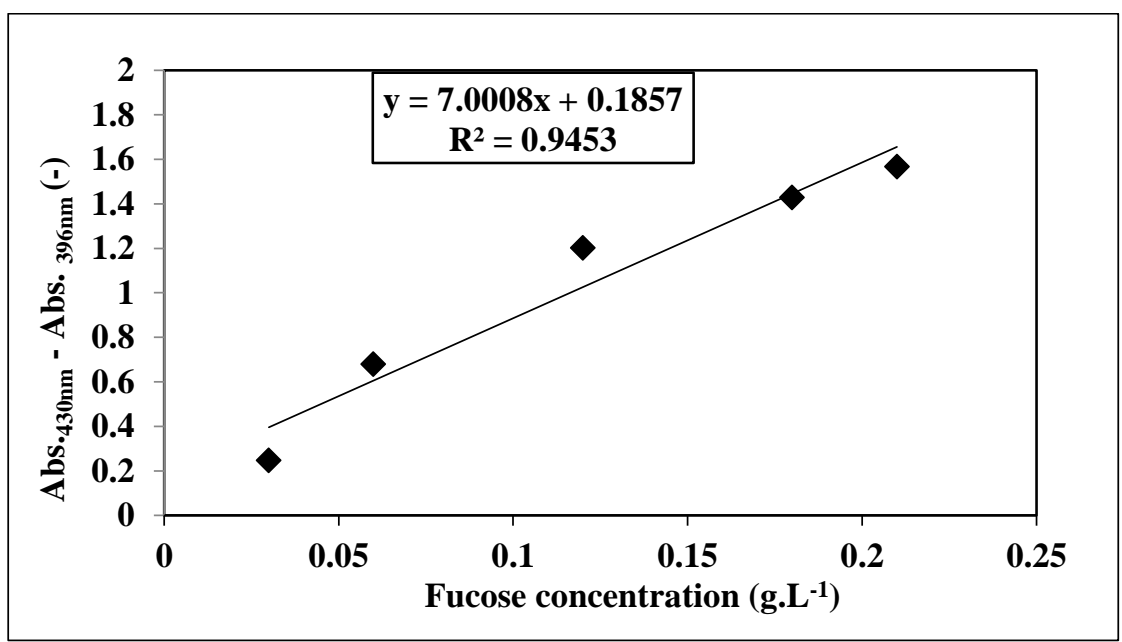

Fig. 78: Calibration curve of Dische assay

L-fucose was used as a standard. Absorbace was measured at two $\lambda$ and the differences were plotted against fucose concentration. 
1.4. Sulphate content in crude fucoidan

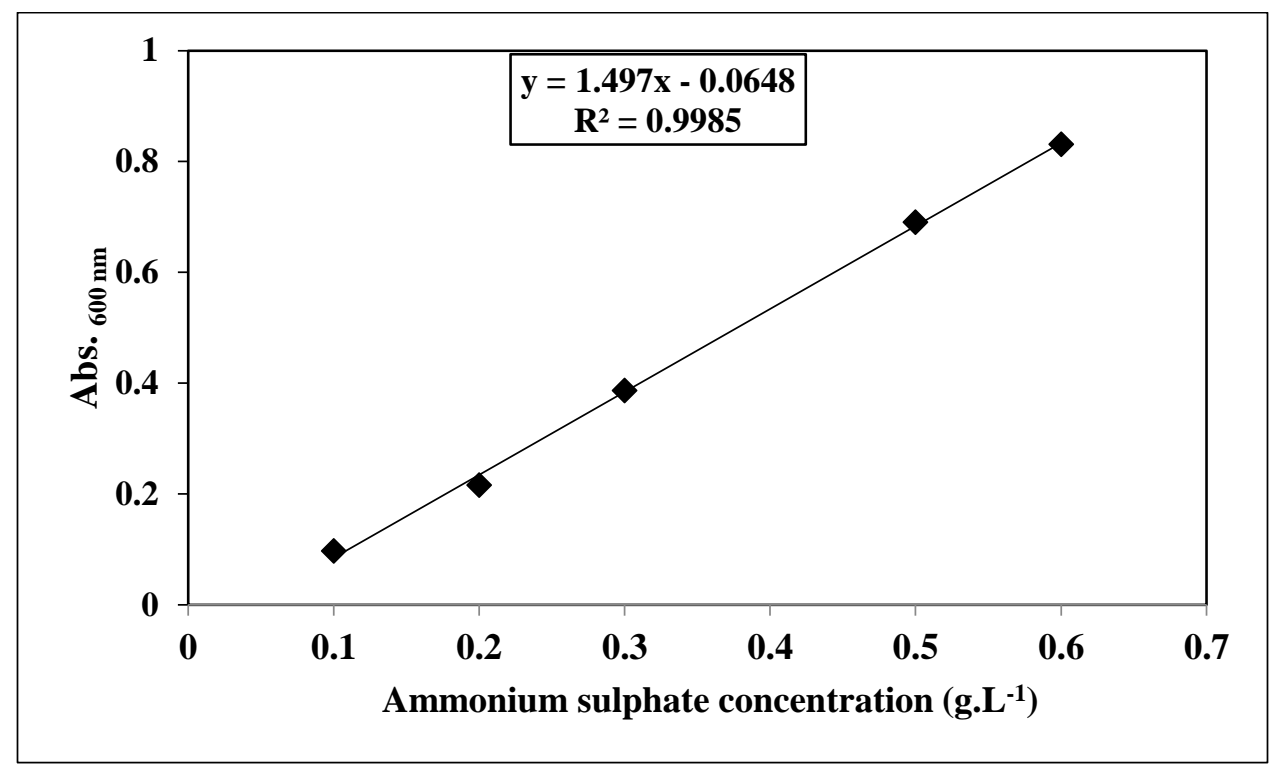

Fig. 79: Calibration curve of $\mathrm{BaSO}_{4}$ test

Ammonium sulphate was used as a standard and absorbance was measured at $600 \mathrm{~nm}$.

1.5. Phosphate standard calibration curve for FucTs activity assay

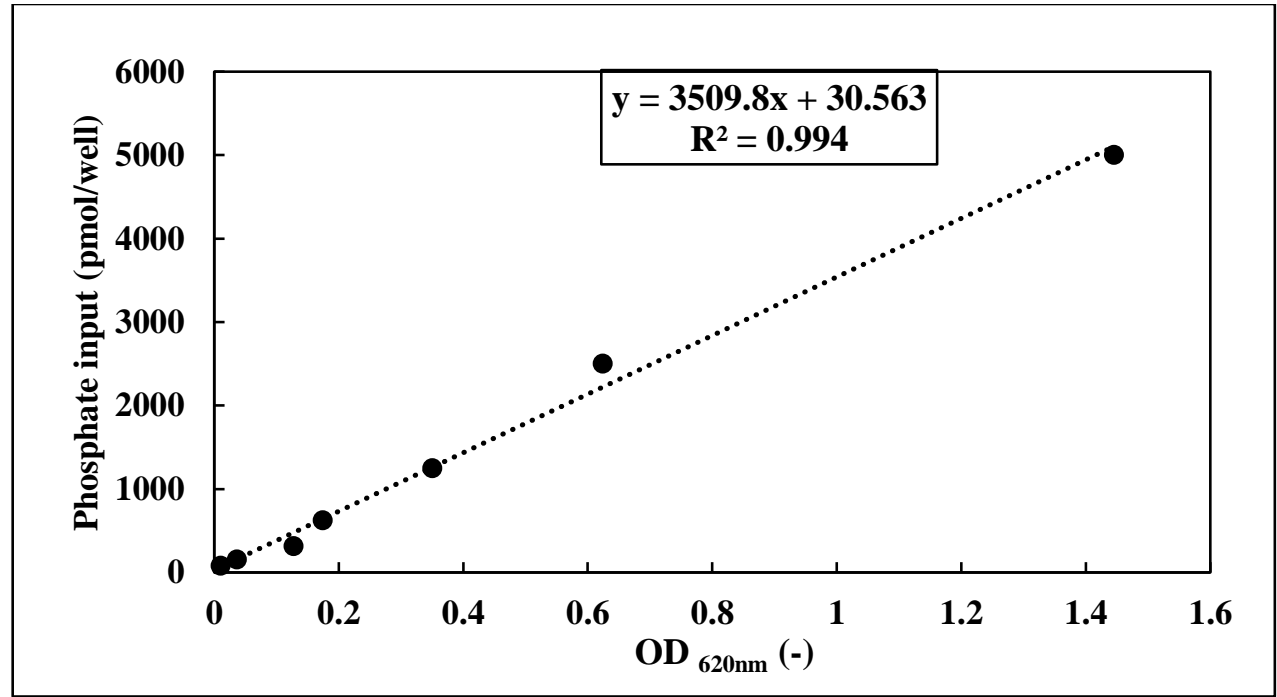

Fig. 80: Phosphate standard calibration curve for FucTs activity assay

Detection was performed by malachite green assay using $\mathrm{KH}_{2} \mathrm{PO}_{4}$ as a standard, following the provider's manual. The slope of the curve was used to determine the specific activity of FucTs_50 as stated in the Glycosylation activity kit's manual. 
1.6. Phosphate standard calibration curve for STs activity assay

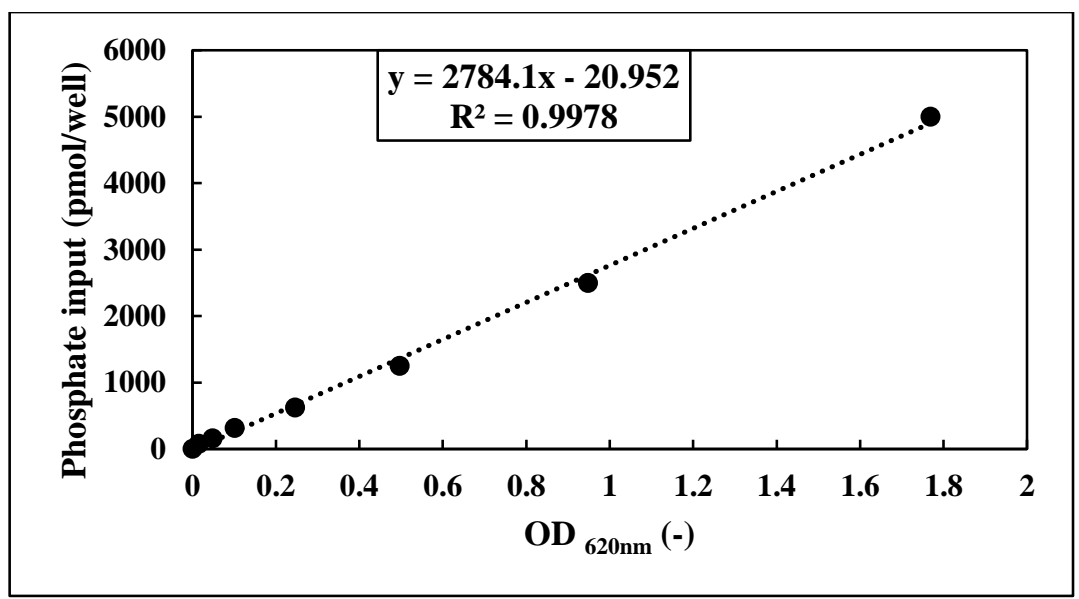

Fig. 81: Phosphate standard calibration curve for STs activity assay

Detection was performed by malachite green assay using $\mathrm{KH}_{2} \mathrm{PO}_{4}$ as a standard. The slope of the curve was used to determine the specific activity of FucTs_50 as stated in the Universal sulphotransferase activity kit's manual.

\section{3D structure of over-expressed algal FucTs and STs}

3D structure of both enzymes were traced by the online free service Phyre ${ }^{2}$ and PyMOL software, as shown in Fig. 82 and $\mathbf{8 3}$ to show their building units and folding. 


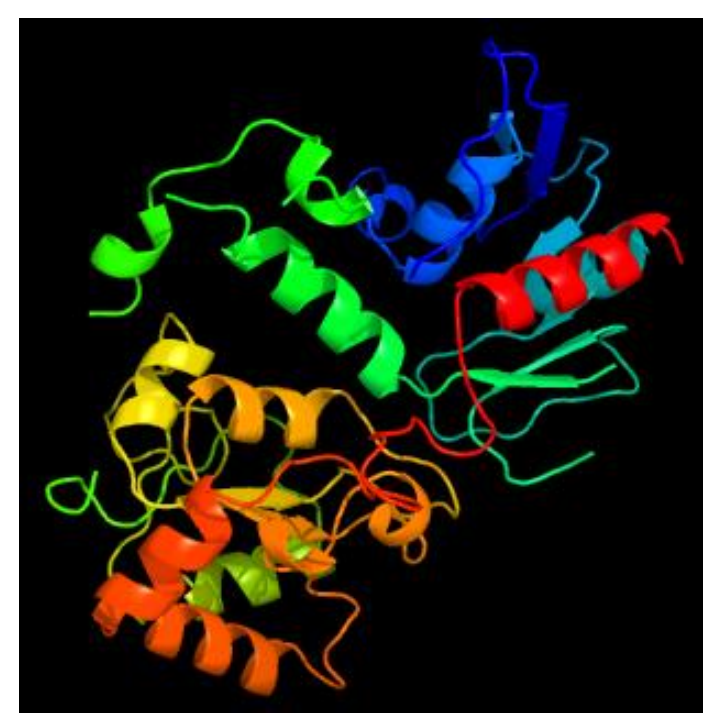

a

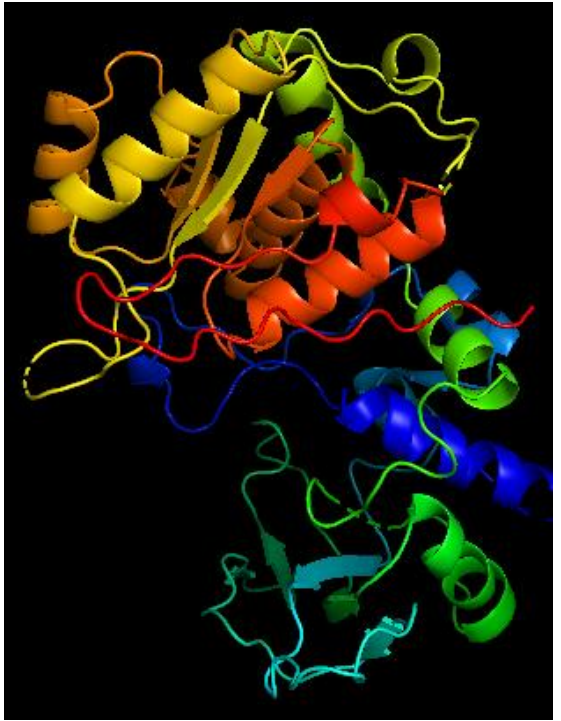

b

Fig. 82: 3D structures of FucTs_50 (a) and FucTs_21 (b) as traced by the free online Phyre ${ }^{2}$ server and PyMOL software

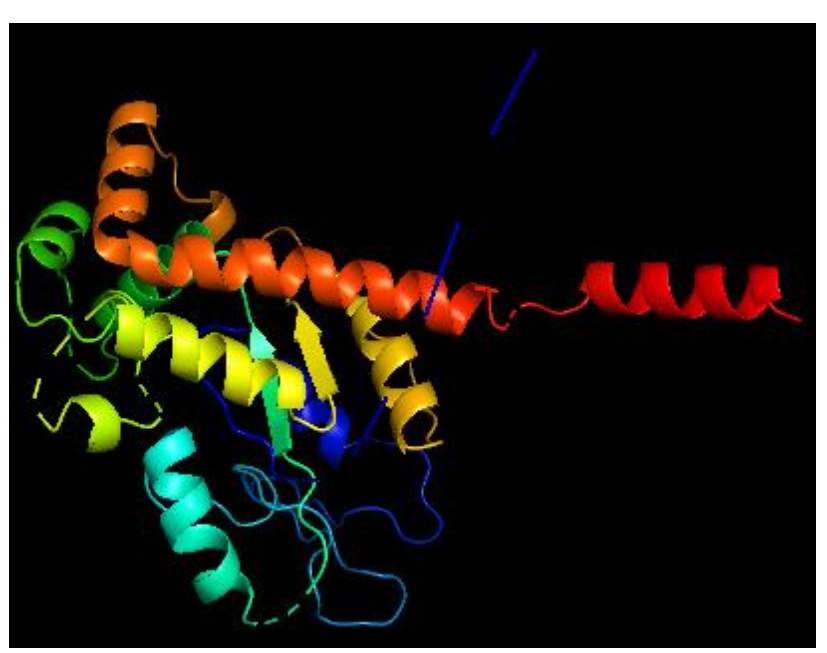

a

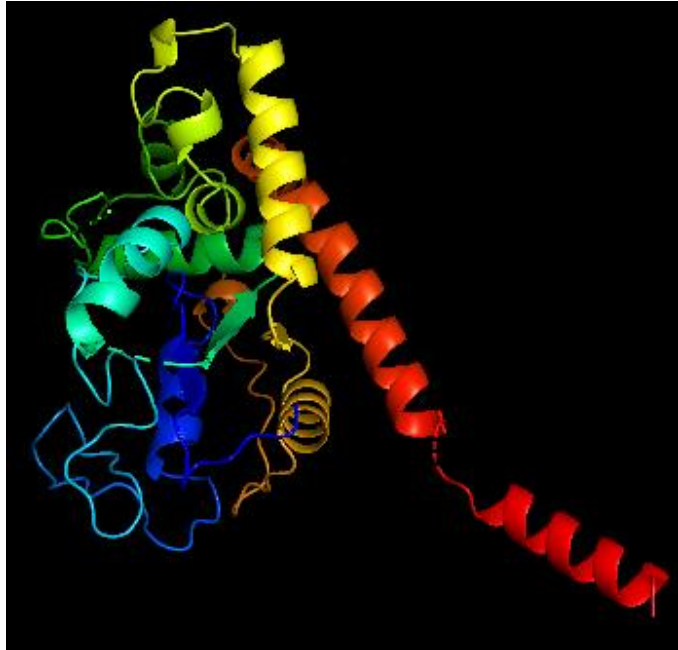

b

Fig. 83: 3D-structures of STs_32 (a) and STs_283 (b) as traced by traced by the online service Phyre ${ }^{2}$ and PyMOL software 


\section{Instruments' charts}

3.1. Automated purification of fucoidan by immobilized PDD

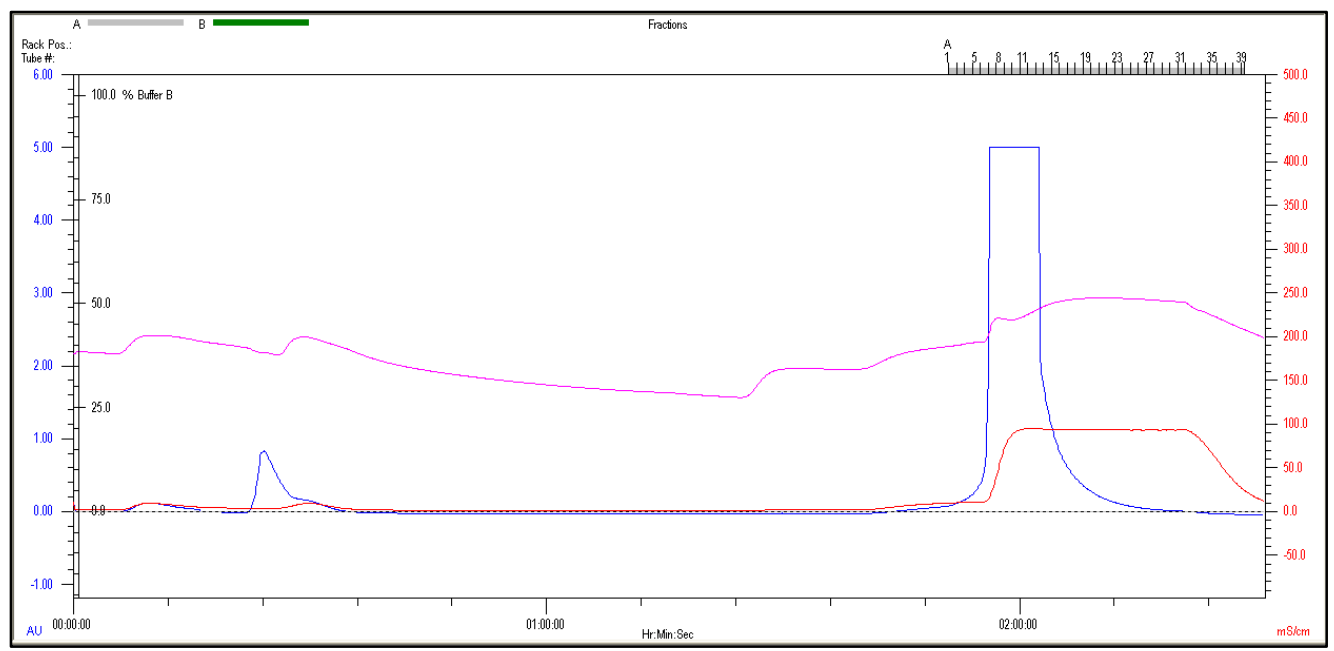

$\mathbf{a}$

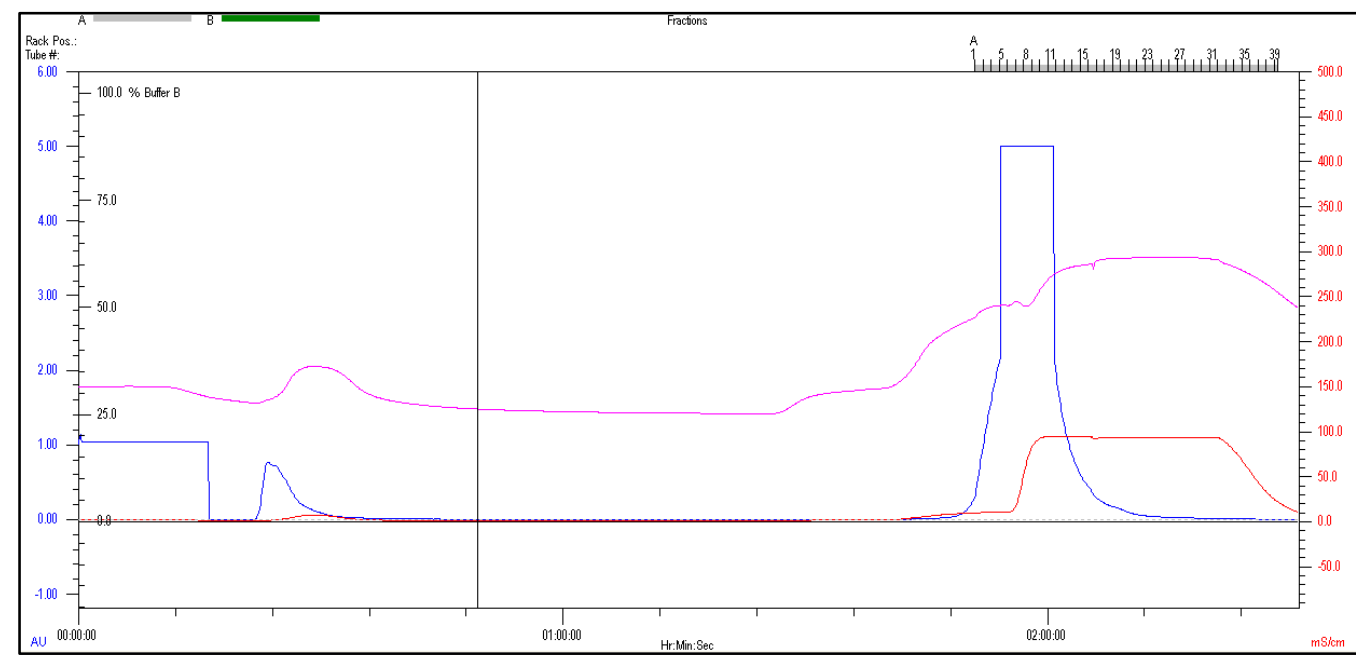

b

Fig. 84: FPLC chromatograms for fucoidan purification by immobilized PDD

a: $1^{\text {st }}$ cycle of purification; $\mathbf{b}: 2^{\text {nd }}$ cycle of purification. Blue line $=U V$ absorbance at $280 \mathrm{~nm}$; Red line $=$ conductivity, Pink line $=\mathrm{pH}$ 
3.2. ${ }^{1} \mathrm{H}-\mathrm{NMR}$ spectra of purified fucoidan

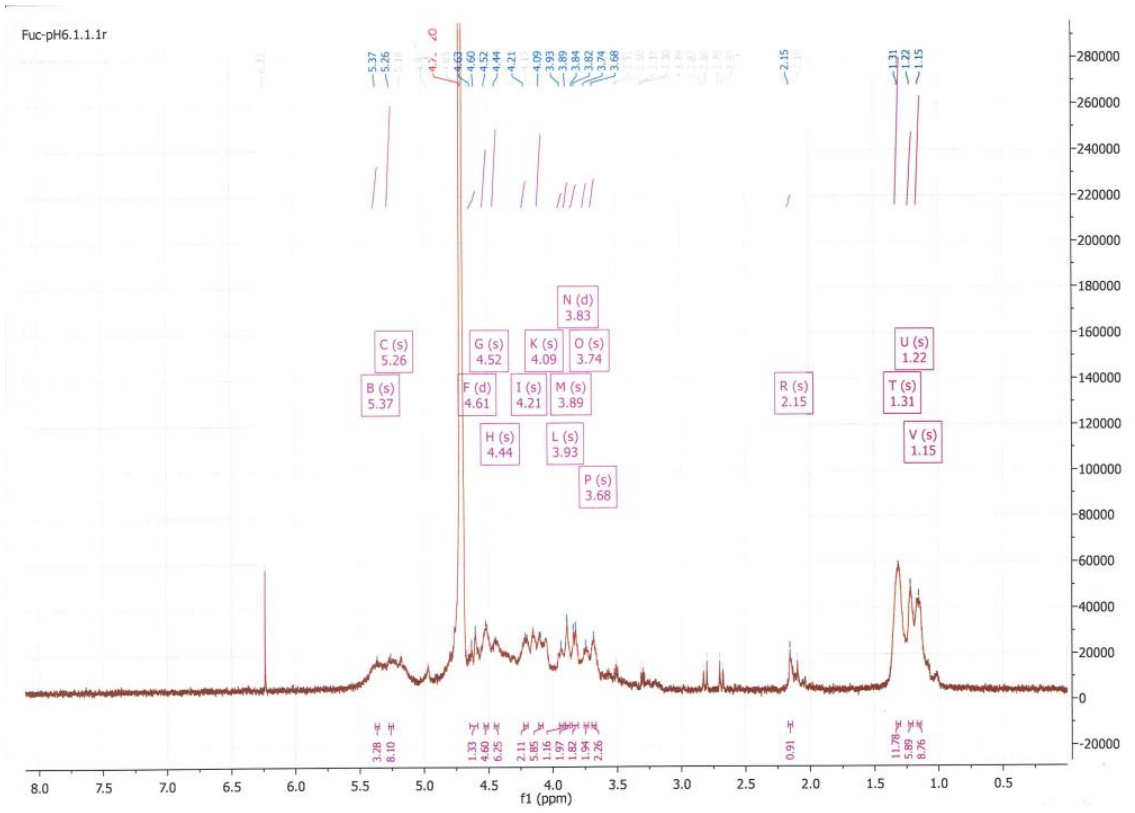

a

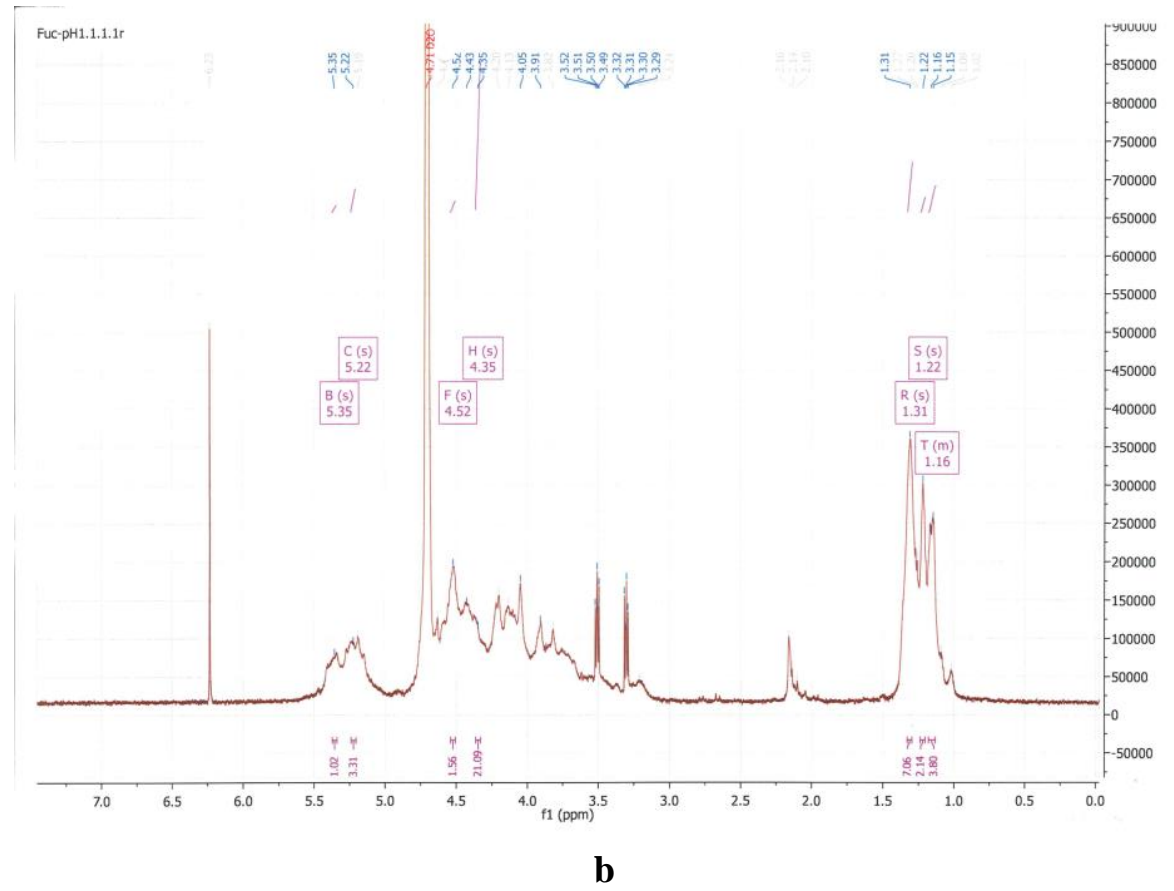

Fig. 85: ${ }^{1} \mathrm{H}-\mathrm{NMR}$ spectra of fucoidan fractions in comparison with fucoidan purchased from SigmaAldrich $^{\circledR}$

Analysis resulted in poorly-translated spectra and revealed in complex nature of fucoidan. (400 $\mathrm{MHz}$, in $\mathrm{D}_{2} \mathrm{O}$ ); a: Fucoidan_6; b: Fucoidan_1; c: Fucoidan_M; d: commercial fucoidan 

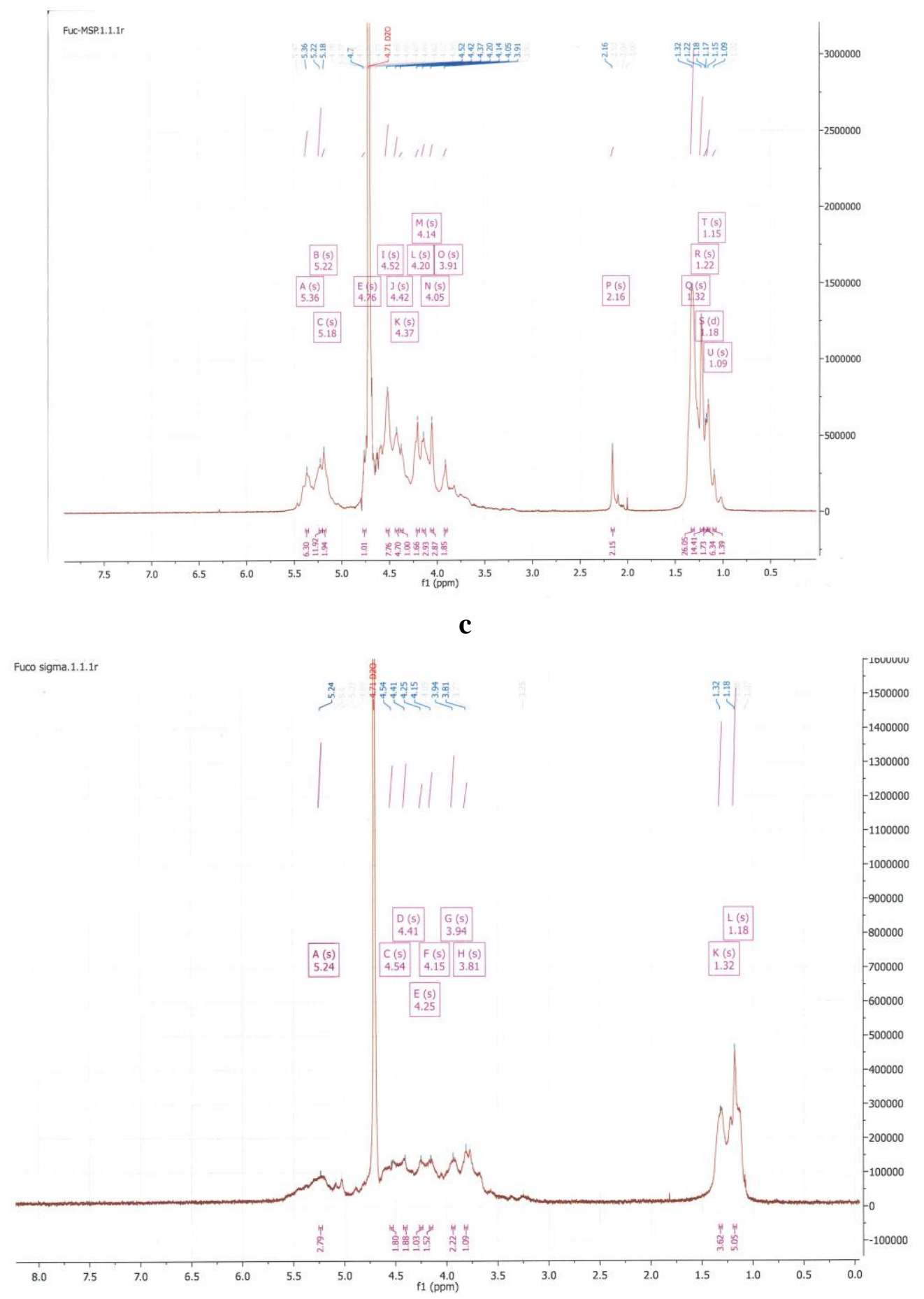

d

Cont., Fig. 85: ${ }^{1} \mathrm{H}$-NMR spectra of fucoidan fractions in comparison with fucoidan purchased from Sigma-Aldrich ${ }^{\circledR}$.

Analysis resulted in poorly-translated spectra and revealed in complex nature of fucoidan. (400 MHz, in $\left.\mathrm{D}_{2} \mathrm{O}\right)$; a: Fucoidan_6; b: Fucoidan_1; c: Fucoidan_M; d: commercial fucoidan 


\section{Others}

4.1. Anti-microbial activity of fucoidan

4.1.1. Anti-fungal activity

Fig. 86 demonstrated the results of the studied anti-fungal activity of fucoidan fractions and commerciallyavailable product against $C$. albicans in comparison with Ampho B. For C. troicalis and glabrata, fucoidan fractions showed a similar pattern and confirmed its inactivity.

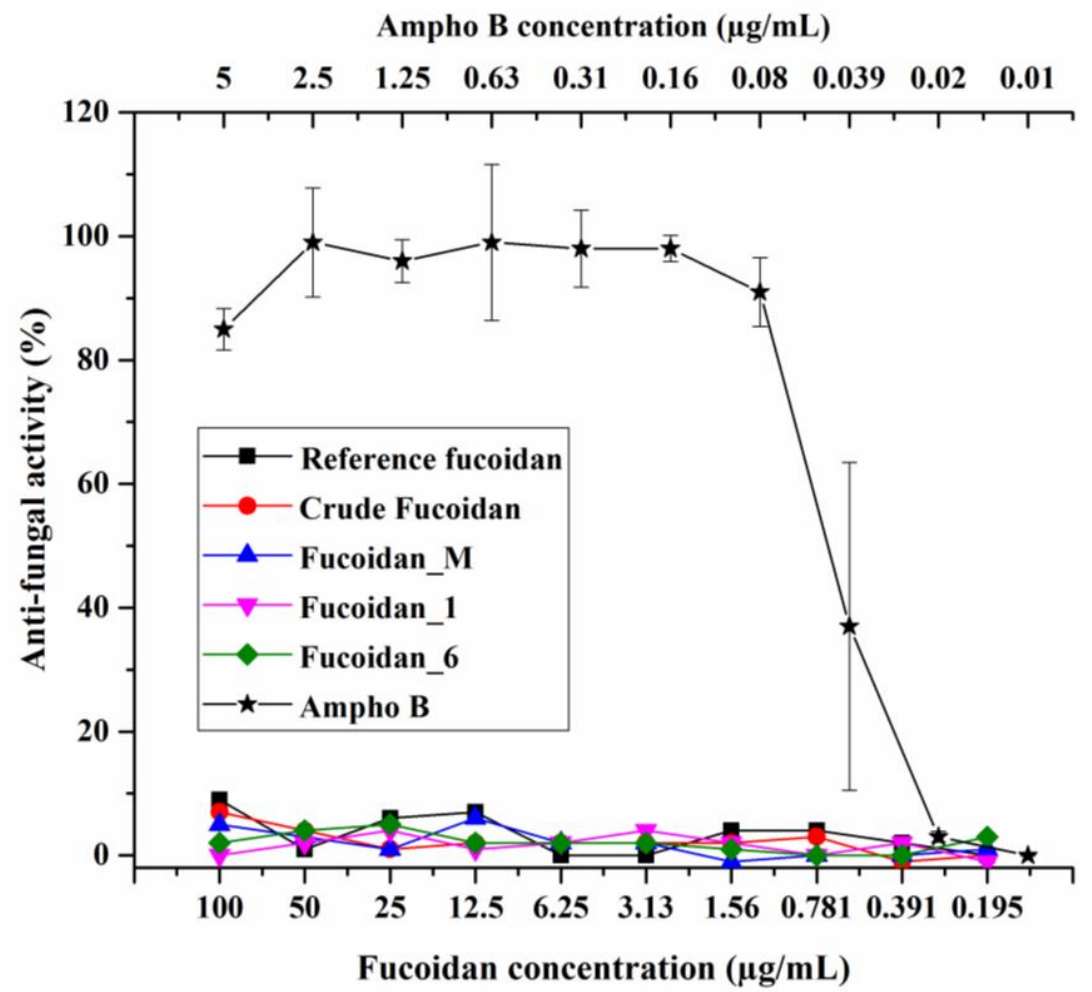

Fig. 86: Anti-fungal activity of different fucoidan fractions against $C$. albicans, in comparison with the reference commercial product following the protocol of Kleymann and Werling [115]

The anti-fungal drug Ampho B was used as a positive control. The figure confirmed inactivity of fucoidan as anti-fungal agent. 


\subsubsection{Anti-bacterial activity}

As shown in Fig. 87, there were no anti-bacterial activity of fucoidan fractions against Gram +ve and G-ve bacteria. Interestingly, in anti-Gram +ve activity assay, with high fucoidan concentration, the viability, however, was increasing and might prove the nourishment of fucoidan for bacteria.

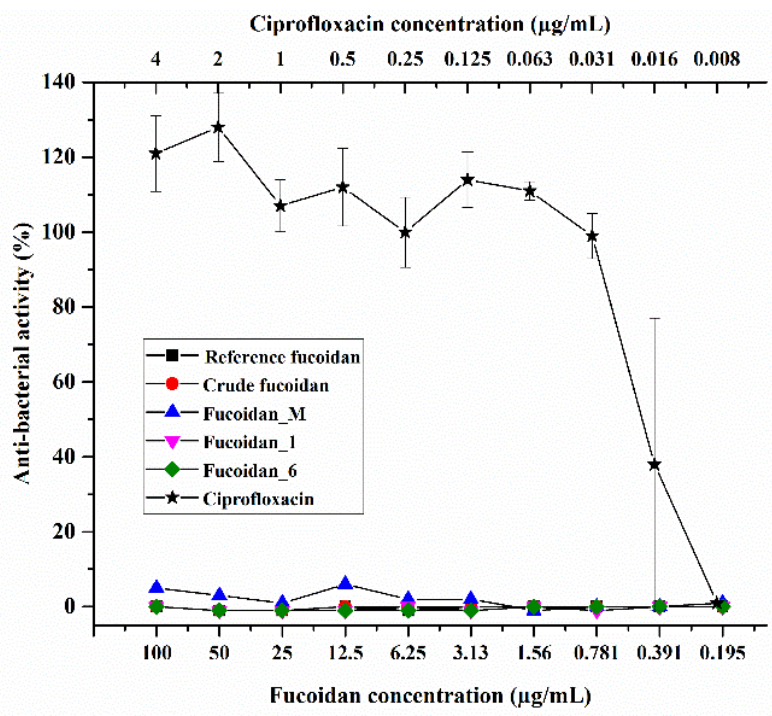

a

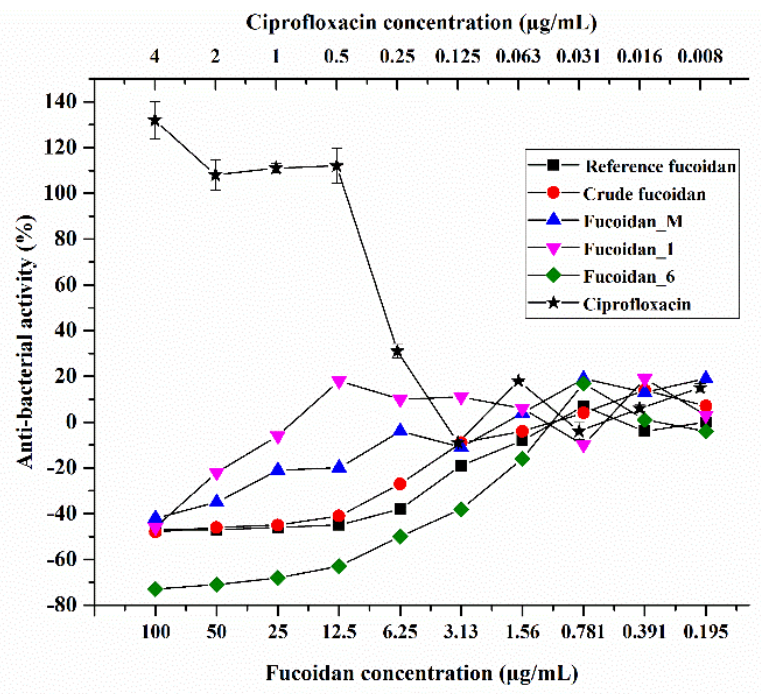

b

Fig. 87: Anti-bacterial activity of different fucoidan fractions against $E$. coli (a) and $S$. aureus (b) in comparison with the reference commercial product and following the protocol of Kleymann and Werling [115]

The anti-bacterial ciprofloxacin was used as a positive control. The figure confirmed inactivity of fucoidan as an anti-bacterial agent. 


\section{2. $\mathrm{CHNS}$ analysis of fucoidan fractions}

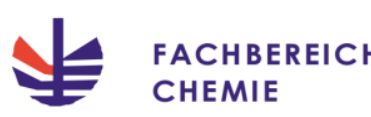

Birgit Dusch

FB Chemie / TU Kaiserslautern

Erwin-Schrödinger-Strasse 54

67663 Kaiserslautern

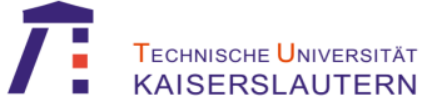

Analytik - Organische Chemie

Tel.: 0631-205-2477

FAX: 0631-205-3921

e-mail: dusch@chemie.uni-kl.de

Technische Universität Kaiserslautern

FB Maschinenbau und Verfahrenstechnik

Lehrgebiet Bioverfahrenstechnik

Prof. Dr. rer. nat. R. Ulber

z.Hd. Herrn M. Sc. Ahmed Zayed

67663 Kaiserslautern

Mittwoch, 19. November 2014

\section{Analysebericht:}

\section{CHNS- Analyse}

Probenbezeichnung: Fuc. (Sigma), Fuc. 40\%, Fuc. 70\%_1, Fuc. 70\%_2

Eingangsdatum: $\quad$ 19.11.2014

Prüfverfahren: $\quad$ in Anlehnung an DIN EN 51732

Interne LA-Nr.: 141111

\begin{tabular}{|c|c|c|c|c|}
\hline Probe & N [\%] & C [\%] & H [\%] & S [\%] \\
\hline Fuc. (Sigma) & - & 24,78 & 4,42 & 8,89 \\
\hline Fuc. 40\% & - & 18,38 & 3,89 & 12,26 \\
\hline Fuc. 70\%_1 & - & 24,61 & 4,79 & 8,16 \\
\hline Fuc. 70\%_2 & 0,55 & 23,42 & 4,22 & 6,78 \\
\hline
\end{tabular}

Kaiserslautern, den 19.11.2014

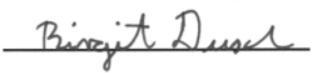

(Birgit Dusch) 
FACHBEREICH CHEMIE

Birgit Dusch

FB Chemie / TU Kaiserslautern

Erwin-Schrödinger-Strasse $\mathbf{5 4}$

67663 Kaiserslautern

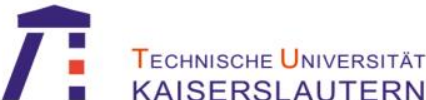

Analytik - Organische Chemie

Tel.: 0631-205-2477

FAX: 0631-205-3921

e-mail: dusch@chemie.uni-kl.de

Technische Universität Kaiserslautern

FB Maschinenbau und Verfahrenstechnik

Lehrgebiet Bioverfahrenstechnik

Prof. Dr. rer. nat. R. Ulber

z.Hd. Herrn M. Sc. Ahmed Zayed

67663 Kaiserslautern

Dienstag, 25. November 2014

\section{Analysebericht:}

\section{CHNS- Analyse}

Probenbezeichnung: Fuc. (Sigma), Fuc. 40\%, Fuc. 70\%_1, Fuc. 70\%_2

Eingangsdatum: $\quad$ 19.11.2014

Prüfverfahren: $\quad$ in Anlehnung an DIN EN 51732

Interne LA-Nr.: 141113

\begin{tabular}{|c|c|c|c|c|}
\hline Probe & $\mathrm{N}[\%]$ & $\mathrm{C}[\%]$ & $\mathrm{H}[\%]$ & $\mathrm{S}[\%]$ \\
\hline Fuc. (pH1) & 0,30 & 24,14 & 4,43 & 11,18 \\
\hline Fuc. (pH6) & 0,34 & 26,12 & 4,63 & 9,83 \\
\hline Fuc. (MSP) & 0,26 & 23,31 & 4,28 & 12,11 \\
\hline
\end{tabular}

Kaiserslautern, den 19.11.2014

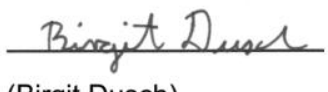

(Birgit Dusch) 


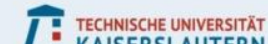

\section{Chemie}

Analytik-Organische Chemie

AG Prof. Dr.-Ing. J. Hartung

Erwin-Schrödinger-Str. 54

67663 Kaiserslautern

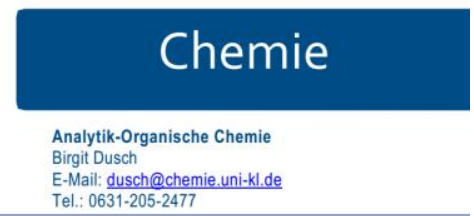

TU Kaiserslautern

FB Maschinenbau und Verfahrenstechnik

Lehrgebiet Bioverfahrenstechnik

Prof. Dr. rer. nat. R. Ulber

z.Hd. Herrn Ahmed Zayed

67663 Kaiserslautern

\section{Analysebericht}

Dienstag, 20.06.2017

Projekt: $\quad$ Elementaranalyse von 3 Proben

Probeneingang: $\quad$ 19.06.2017

Int. Auftragsnummer: 170608

Probenbezeichnung: Crude fucoidan, DDP-1 fucoidan, DDP-2 fucoidan

Probenvorbereitung: nicht erforderlich

Prüfmethode: $\quad$ Verbrennungsanalyse nach DIN $51732(\mathrm{CHN})$ bzw. DIN $15178(\mathrm{~S})$

Prüfgerät:

Vario Micro Cube, Firma Elementar

Ergebnisse (Mittelwerte):

\begin{tabular}{|l|c|c|c|c|}
\hline Probe & N [\%] & C [\%] & H [\%] & S [\%] \\
\hline Crude fucoidan & 0,59 & 22,89 & 4,54 & 6,88 \\
\hline DDP-1 fucoidan & 0,30 & 25,77 & 4,57 & 8,78 \\
\hline DDP-2 fucoidan & 0,29 & 25,95 & 4,54 & 8,78 \\
\hline
\end{tabular}

Anmerkung:

$$
\begin{aligned}
& \text { Von allen Proben wurden Doppel- bzw. Dreifachmessungen } \\
& \text { durchgeführt. Die angegebenen Werte sind Mittelwerte. Probe Crude } \\
& \text { fucoidan sieht inhomogen aus, deshalb wurde eine Dreifachmessung } \\
& \text { durchgeführt. Die Werte zeigen aber gute Übereinstimmung. } \\
& \text { Einzelergebnisse siehe beigefügte Excel-Datei. }
\end{aligned}
$$

Kaiserslautern, den 20.06.2017

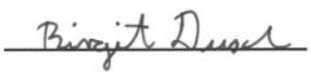

(Birgit Dusch)

Page 1 from 1 
TECHNISCHE UNIVERSITÄT
KAISERSLAUTERN

Analytik-Organische Chemie

AG Prof. Dr.-Ing. J. Hartung

Erwin-Schrödinger-Str. 54

67663 Kaiserslautern

TU Kaiserslautern

FB Maschinenbau und Verfahrenstechnik

Lehrgebiet Bioverfahrenstechnik

Prof. Dr. rer. nat. R. Ulber

z.Hd. Herrn Ahmed Zayed

67663 Kaiserslautern

\section{Chemie}

Analytik-Organische Chemie

Birgit Dusch

E-Mail: dusch@chemie.uni-kl.de

Tel.: 0631-205-2477

\section{Analysebericht}

Montag, 06.03.2017

Projekt: Elementaranalyse von 1 Probe

Probeneingang: $\quad 06.03 .2017$

Int. Auftragsnummer: 170302

Probenbezeichnung: Fucoidan-FDD6

Probenvorbereitung: nicht erforderlich

Prüfmethode: $\quad$ Verbrennungsanalyse nach DIN $51732(\mathrm{CHN})$ bzw. DIN 15178 (S)

Prüfgerät: $\quad$ Vario Micro Cube, Firma Elementar

\section{Ergebnisse:}

\begin{tabular}{|l|l|l|l|l|}
\hline Probe Fucoidan-FDD6 & N [\%] & C [\%] & H [\%] & S [\%] \\
\hline & $<0.5$ & 25,35 & 4.51 & 8,45 \\
\hline
\end{tabular}

\section{Anmerkung:}

Kaiserslautern, den 06.03.2017

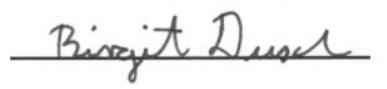

(Birgit Dusch) 


\subsection{Melting point analysis of fucoidan fractions}

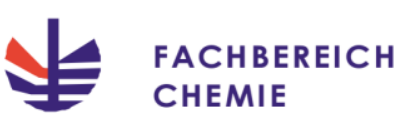

Ruth M. Bergsträßer

FB Chemie / TU Kaiserslautern Erwin-Schrödinger-Strasse 54 67663 Kaiserslautern
TECHNISCHE UNIVERSITÄT

KAISERSLAUTERN

Analytik - Organische Chemie

Tel.: 0631-205-2965

FAX: 0631-205-3921

e-mail: rbergstr@rhrk.uni-kl.de

Technische Universität Kaiserslautern

FB Maschinenbau und Verfahrenstechnik

02.12 .2014

Lehrgebiet Bioverfahrenstechnik

Prof. Dr. rer. nat. R. Ulber

z.Hd. Herrn M. Sc. Ahmed Zayed

67663 Kaiserslautern

Internal order number: 141113-II

\section{Report: Melting Point- Analysis}

Sample name:

Date of receipt:

Method:
Fuc. (MSP), Fuc. (pH 1), Fuc. (pH 6)

19.11.2014, ordering: 25.11 .2014

Melting Point Apparatus: DigiMelt-MPA160, SRS,

Scientific Instruments $\mathrm{GmbH}$; Ramp $2 \% \mathrm{~min}$;

Sense:

\begin{tabular}{|c|l|c|}
\hline Sample & \multicolumn{1}{|c|}{ Description } & Quantity $[\mathrm{g}]$ \\
\hline Fuc. (pH1) & white, lighty flakes, fluffy, very easy to pour into the capillary & ca. $0,035 \mathrm{~g}$ \\
\hline Fuc. (pH6) & white flakes, like cotton, difficult to pour into the capillary & ca. $0,020 \mathrm{~g}$ \\
\hline Fuc. (MSP) & Cream white, foliated, easy to pour into the capillary & ca. $0,061 \mathrm{~g}$ \\
\hline
\end{tabular}

Results:

\begin{tabular}{|c|c|c|c|}
\hline Sample & $\begin{array}{c}\text { Starting Temperatur } \\
{\left[{ }^{\circ} \mathrm{C}\right]}\end{array}$ & $\begin{array}{c}\text { Beginning of yellowing } \\
{\left[{ }^{\circ} \mathrm{C}\right]}\end{array}$ & $\begin{array}{c}\text { Decomposition point } \\
{\left[{ }^{\circ} \mathrm{C}\right]}\end{array}$ \\
\hline Fuc. $(\mathrm{pH} 1)$ & 130 & $132-133$ & 135 \\
\hline Fuc. (pH6) & 140 & $153-156$ & 161 \\
\hline Fuc. (MSP) & 130 & $133-134$ & 136 \\
\hline
\end{tabular}

Remark: Many organic substances are melting under decomposition. This is often evidenced by decoloration from yellow up to brown and black, often reflected by gas development. This decomposition point is often diffuse and not exactly reproducible. Some substances are carbonizing.

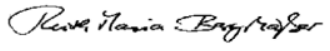

Ruth Maria Bergsträßer, (Dipl.Chem.) 


\subsection{Specific optical rotation of fucoidan fractions}

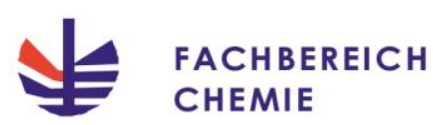

Ruth M. Bergsträßer

FB Chemie / TU Kaiserslautern

Erwin-Schrödinger-Strasse 54 67663 Kaiserslautern

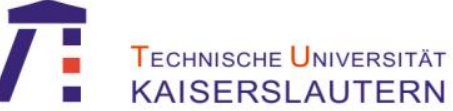

Analytik - Organische Chemie

Tel.: 0631-205-2965

FAX: 0631-205-3921

e-mail: rbergstr@rhrk.uni-kl.de

Technische Universität Kaiserslautern

FB Maschinenbau und Verfahrenstechnik

15.12 .2014

Lehrgebiet Bioverfahrenstechnik

Prof. Dr. rer. nat. R. Ulber

z.Hd. Herrn M. Sc. Ahmed Zayed

67663 Kaiserslautern

\section{Report: Specific Optical Rotation- Analysis}

Sample name: $\quad$ Standard Fucoidan from Fucus vesiculosus $\geq 95 \%$, Sigma Aldrich;

Fuc. (MSP); Fuc. (pH 1); Fuc. (pH 6)

Date of receipt: $\quad$ 19.11.2014, ordering: 03.12.2014

Method: P-2000 Digital Polarimeter, Fa. Jasco

Measurement details: sodium spectral lamp with $\Lambda=589 \mathrm{~nm}$; path length $=100 \mathrm{~mm}$;

temperature $=22^{\circ} \mathrm{C}$; solvent $=$ distilled water

concentration of the solutions $=0,4[$ weight $/$ volume $\%]$;

Results:

\begin{tabular}{|l|c|}
\hline Sample & $\begin{array}{c}\text { Specific O.R. } \\
{[\alpha]_{589}^{22} ; \mathrm{C}=0.4 ; \mathrm{H}_{2} \mathrm{O}_{\text {dest }}}\end{array}$ \\
\hline Standard Fucoidan & -121 \\
\hline Fuc. (pH1) & -128 \\
\hline Fuc. (pH6) & -117 \\
\hline Fuc. (MSP) & -130 \\
\hline
\end{tabular}

Reveriaria Bagreagser

Ruth Maria Bergsträßer, (Dipl.Chem.) 


\section{Appendix F}

\section{Lists of Figures, Tables, Schemes and devices}

\section{List of Figures}

Fig. No.

\section{Fig. Caption}

1. Seaweed production in the year 2014 by Aquaculture

2. Global distribution of the major brown macroalgae species

3. Overview of the different strategies dealt in the present work

4. Cell wall model in a brown macroalgae showing various fucoidan physiological functions

5. Published articles on fucoidan since 1900 till July 2017, according to ISI web of knowledge (Clarivate Analytics)

6. a: Different chemical structures of fucoidan from some Fucales seaweeds

b: Chemical structures of fucoidan from some Laminariales and Chordariales seaweeds

7. Steps of immobilization protocol of TB on amino derivatized Sepabeads ${ }^{\circledR}$ EC-EA

8. Habitat of $F$. vesiculosus or bladder wrack across the north Atlantic in more temperate zone

9. Morphology and anatomical parts of $F$. vesiculosus thallus

10. Growth of the brown macroalgae $F$. vesiculosus at the south beaches of Wilhelmshaven (North Sea, $53^{\circ} 31.236 \mathrm{~N}, 8^{\circ} 13.849 \mathrm{E}$, Germany)

11. Extraction set of fucoidan from dried pretreated $F$. vesiculosus

12. Overview of fucoidan extraction process from pre-treated $F$. vesiculosus biomass and obtained crude fucoidan

13. Overview for isolated fractions of purified fucoidan

14. Average composition of the dried biomass of $F$. vesiculosus

15. Representation of polyanionic polysachharide reaction with fluorescent Heparin Red $^{\circledR}$

16. Adsorption (\%) of fucoidan by immobilized $2 \mathrm{mM} \mathrm{TB}$, TA and mixed dyes at $\mathrm{pH} 1$ and $\mathrm{pH} 6$

17. Comparison between adsorption (\%) of fucoidan by immobilized 2, 4 and $6 \mathrm{mM}$ TB at pH 1

18. Adsorption (\%) of fucoidan by $2 \mathrm{mM}$ immobilized $\mathrm{TB}$ for $60 \mathrm{~h}$ of incubation at $\mathrm{pH} 1$ and $\mathrm{pH}$ 6

19. Adsorption (\%) of fucoidan from Fucoidan_A by $75 \mathrm{mg}$ of $2 \mathrm{mM}$ immobilized TB at pH 1 and pH 6 


\section{Fig. No.}

\section{Fig. Caption}

20. Adsorbed fucoidan (\%) by $75 \mathrm{mg}$ of immobilized TB for two cycles at $\mathrm{pH} 1$ and $\mathrm{pH} 6$

21. Effect of $\mathrm{NaCl}$ molarity in eluent on eluted fucoidan (\%)

22. Immobilized TB and TA on Sepabeads ${ }^{\circledR}$ EC-EA

23. Molecular structure of a) perylene diimide derivative ( $N, N^{\prime}$-Bis-(1-amino-4,9-diaza dodecyl)1,7-di bromo perylen-3,4:9,10-tetracarboxylic acid diimide, and b) Heparin $\operatorname{Red}^{\circledR}$

24. Immobilized PDD on Sepabeads ${ }^{\circledR}$ EC-EA

25. Adsorption (\%) of fucoidan from Fucoidan_A by immobilized PDD

26. Multiple use of PDD-derivatized beads for three cycles

27. Fucoidan elution pattern from FPLC column in two successive cycles

28. $\quad$ FT-IR spectra of fucoidan before (Fucoidan_crude) and after (Fucoidan_PDD) in comparison with the commercially-available reference fucoidan purchased from Sigma-Aldrich ${ }^{\circledR}(>95 \%$ pure)

29. Representation of an $\alpha$-(1-3)-linked L-fucopyranoside repeating unit of fucoidan, as previously described by Cumashi, et al.

30. a: Effect of different fucoidan fractions on aPPT at a concentration of $0.01 \mathrm{mg} \mathrm{mL}^{-1}$ b: A dose-dependent effect of Fucoidan_PDD on aPTT

31. Effect of different types of fucoidan on PT at a concentration of $0.01 \mathrm{mg} \mathrm{mL}^{-1}$

32. a: Effect of different fucoidan fractions on TT at a concentration of $0.01 \mathrm{mg} \mathrm{mL}^{-1}$

b: A dose-dependent anti-thrombin effect of Fucoidan_PDD

33. Comparison between the anti-viral activities of fucoidan fractions against HSV-1

34. Antioxidant activity of different fucoidan fractions in comparison with ascorbic acid

35. Downstream process for fucoidan extraction and purification by either TB- or PDD-derivatized beads at $\mathrm{pH} 6$

36. Determination of Fucoidan_PDD purity using Heparin Red ${ }^{\circledR}$ Ultra assay, in comparison with the commercial standard product ( $>95 \%$ pure) purchased from Sigma-Aldrich ${ }^{\circledR}$

37. Callus and plantlet regeneration from an intact marine macroalgae thallus of Agardhiella subulata

38. Summary of previously-applied growth conditions and variables to develop marine macroalgal cultures

39. Applied growth conditions and variables to induce callus-like and protoplast cultures

40. Different explants of F. vesiculosus incubated on MB50 (a, b, c, d and e) and LB (f) media at $17^{\circ} \mathrm{C}$ and $26^{\circ} \mathrm{C}$, respectively, after different steps of Protocol 6 


\section{Fig. No.}

\section{Fig. Caption}

41. Enzymatic reduction of tetrazolium chloride (TTC) (Tetrazolium cation, colorless) to triphenylformazan (TPF) (Formazan, red color)

42. Protoplasts after $72 \mathrm{~h}$ heterotroph cultivation in the protoplast medium showing start of cell multiplication and aggregation

43. Development of a phototrophic callus-like growth from F. vesiculosus explant in ASP-12NTA medium

44. (a) Cultivation of F. vesiculosus explants in a wave bag bioreactor, (b) Filamentous callus-like growth from F. vesiculosus explant developed after four weeks of phototrophic cultivation in PES medium

45. Development of crown gall or hairy-root in F. vesiculosus explants after transfection with $R$. radiobacter or A. tumefaciens DSM 30147

46. The two different possible pathways for fucoidan biosynthesis in the brown algae Ectocarpus siliculosus (a) De novo pathway and b) salvage pathway

47. Detailed de novo (a) and salvage pathway (b) for GDP-L-fucose biosynthesis either from GDPmannose or cytosolic L-fucose, respectively

48. Synthetic and cloned Esi0050_0098 and Esi0021_0026 in pMA-T and pMK plasmid vectors, respectively

49. Features of pASK-IBA 45(+) plasmid vector as described by IBA GmbH

50. Homology and phylogenetic relationships of algal FucTs_21 and FucTs_50 with other bacterial and human FucTs

51. Gibson Assembly work flow; an example

52. Agarose gel electrophoresis of amplified PCR products of Esi0021_0026 and Esi0050_0098

53. Design of pASK-IBA 45(+)_Esi0021_0026 DNA construct showing some possible restriction sites and gel electrophoresis results after its digestion with $S t u \mathrm{I}$ and $\mathrm{XbaI}$

54. Design of pASK-IBA 45(+)_Esi0050_0098 DNA construct showing some possible restriction enzymes and gel electrophoresis results after digestion $\mathrm{XbaI}$

55. Alignment of DNA templates (pASK_Esi0021 and pASK_0050) with the forward (fwd) sequence of the sequencing results

56. SDS-PAGE (a) and Western blot (b) of overexpressed recombinant proteins from pASK_IBA 45(+)_Esi0021_0026 and pASK_IBA 45(+)_Esi0050_0098 DNA constructs, in comparison with an empty vector plasmid in a $2 \mathrm{~L}$ of LB medium of $E$. coli BL21(DE3)

57. Western blot of expressed recombinant FucTs_50 from the pASK-IBA 45(+)_Esi0050_0098 construct in comparison with empty vector plasmid during purification cascade

58. Glycosyltransferase activity kit principle, as described by the supplier (Bio-Techne ${ }^{\circledR}$ ) 


\section{Fig. No.}

\section{Fig. Caption}

59. Measurement of catalytic activity of FucTs_50 on the donor substrate GDP-L-fucose by Glycosyltransferase activity kit

60. FucTs_50 hydrolytic activity toward GDP-L-fucose as determined by MC-CE

61. Catalysis of sulphonate group (SO3-) transfer to a hydroxyl group-containing compound by Sulphotransferases (STs) using PAPS as a donor substrate

62. Cloned E. coli_opt. Esi0032_0064 and Esi0283_0018 in pMA-T plasmid vector

63. A homology study between algal STs_283, STs_32 and the human Galactose-3-Osulfotransferase 2 (G3ST2)

64. Agarose gel electrophoresis of (a) amplified Esi0032_0064 (1386 bp) and (b) Esi0283_0018 (1032 bp) with their overlapping ends from EcoRI/SacI restriction sites of the vector pASKIBA $45(+)$

65. Designed pASK-IBA 45(+)_Esi0032_0064 (a) and pASK-IBA 45(+)_Esi0283_0018 (b) templates by pDRAW32 DNA analysis software

66. Agarose gel electrophoresis of extracted DNA constructs; pASK-IBA 45(+)_Esi0032_0064_Opt. and pASK-IBA 45(+)_Esi0283_0018_Opt. after digestion with $X b a \mathrm{I}$

67. Alignment of sequencing results of fwd DNA constructs; pASK-IBA 45(+)_Esi0032_0064_Opt. (a) and pASK-IBA 45(+)_Esi0283_0018_Opt. (b) with the designed templates

68. Western blot of purified heterologusly expressed STs_32 by E. coli BL21 (DE3)

69. Western blot of purified heterologusly expressed STs_283 by E. coli BL21 (DE3)

70. Principle of the Universal Sulphotransferase activity kit as described by the supplier (BioTechne ${ }^{\circledR}$ )

71. a: Relationship between purified STs_32 at different concentrations and liberated free phosphate from PAPS detected by malachite green and measured at $620 \mathrm{~nm}$

b: Relationship between purified STs_283 at different concentrations and from PAPS detected by malachite green and measured at $620 \mathrm{~nm}$

72. Simulated fucosylation reaction of GlucNAc catalyzed by heterologously expressed FucTs_50

73. Chemical structures of some selected marine-derived compounds previously-mentioned in Table 1

74. SDS-PAGE plate showing its building components

75. Calibration curve of Dubois or phenol-sulphuric acid assay

76. Calibration curve of TB assay

77. Calibration curve of Heparin $\operatorname{Red}^{\circledR}$ Ultra assay 


\section{Fig. No.}

78. Calibration curve of Dische assay

79. Calibration curve of $\mathrm{BaSO} 4$ assay

80. Phosphate standard calibration curve for FucTs activity assay

81. Phosphate standard calibration curve for STs activity assay

82. 3D structures of FucTs_50 (a) and FucTs_21 (b) as traced by the free online Phyre ${ }^{2}$ server and PyMOL software

83. 3D-structures of STs_32 (a) and STs_283 (b) as traced by traced by the online service Phyre ${ }^{2}$ and PyMOL software

84. FPLC chromatograms for fucoidan purification by immobilized PDD

85. ${ }^{1}$ H-NMR spectra of fucoidan fractions in comparison with fucoidan purchased from SigmaAldrich $^{\circledR}$

86. Anti-fungal activity of different fucoidan fractions against $C$. albicans, in comparison with the reference commercial product following the protocol of Kleymann and Werling

87. Anti-bacterial activity of different fucoidan fractions against E. coli (a) and S. aureus (b) in comparison with the reference commercial product and following the protocol of Kleymann and Werling 


\section{List of Tables}

Table. No.

Table title

1. Selected categories and examples of marine-derived products.

2. Taxonomy of $F$. vesiculosus

3. Description of an automated fucoidan purification process by immobilized PDD using FPLC

4. Sugar, fucoidan, fucose and sulphate contents in different crude extracted fucoidan fractions from $F$. vesiculosus by Procedure_A and C

5. Elemental analysis (CHNS) and degree of sulphation results of different fucoidan fractions

6. a: Molecular weight parameters and polydispersity index (PDI) of different purified fucoidan fractions.

b: Molecular weight parameters of fractions obtained by different $\mathrm{NaCl}$ molarity

7. Comparison among the different fucoidan fractions purified by immobilized TB, regarding start, colour change and decomposition temperature points

8. Comparison among the different fractions of fucoidan regarding specific optical rotation

9. Monomeric composition (\%) of different purified fucoidan fractions

10. IC50 ( $\mu \mathrm{g} . \mathrm{mL}-1)$ of different fucoidan fractions isolated and purified from F. vesiculosus against HSV-1 in comparison with aciclovir

11. Some selected trials with callus cultures and plant regeneration in marine macroalgae organisms

12. Antibiotic stock solution (30x) composition

13. Summary of processes performed in all surface sterilization protocols

14. Examples of previous trials with heterologous expression to overexpress similar enzymes from different resources to that involved in fucoidan biosynthesis

15. Designed primers for cloning of Esi0050_0098 and Esi0021_0026 in pASK-IBA 45(+), according to NEBuilder ${ }^{\circledR}$ Assembly Tool

16. Overview of different algal STs, regarding their names, sizes of encoded genes, number of amino acids and putative functions

17. Designed primers for cloning of E-coli-adapted Esi0032_0064 and Esi0283_0018 in pASKIBA 45(+), according to NEBuilder ${ }^{\circledR}$ Assembly Tool

18. PCR steps used in amplification of synthetic Esi0021_0026, Esi0050_0098, Esi0283_0018 and Esi0032_0064 


\section{List of schemes}

Scheme No.

\section{Scheme caption}

1. Overview of pre-treatment steps of the dried algae biomass before fucoidan extraction

2. Graphical summary for fucoidan purification process from F. vesiculosus 


\section{List of used devices and instruments}

Name of the device

- $\quad$ A Glass column (XK 16/20)

- Analytical balance (KERN ABS)

- $\quad$ Autoclave (Systec V-150)

- Bench centrifuge for eppis (Centrifuge 5415 D)

- Blood coagulation system $\left(\mathrm{BCS}^{\circledR}\right.$ System)

- Capillary electrophoresis (cePRO 9600M)system

- Conductivity meter set (Qcond 2200)

- Cryopreservation refrigerator (Ultra low temperature freezer AV039P)

- Digital polarimeter (P-2000)

- $\quad$ Drying oven

- $\quad$ Electrotansfer cell (Trans-Blot ${ }^{\circledR}$ SD Semi-Dry Transfer Cell)

- Elemental Vario Micro cube apparatus (WLD Board, V 2.0.11)

- FPLC (BioLogic Duo-Flow ${ }^{\mathrm{TM}}$ ) supplied

- $\quad$ Freeze dryer $\left(\right.$ Christ $^{\circledR}$ LDC-1m, Alpha 2-4)

- $\quad$ FT-IR (Spectrum 100)
Company

\section{Country}

GE Healthcare Europe GmbH

Germany

KERN \& SOHN GmbH

Germany

SYSTEC GmbH Labor-

Germany

Systemtechnik

Eppendorf

Germany

Siemens Healthcare Diagnostics

Germany

Products $\mathrm{GmbH}$,

Advanced Analytical Technologies

USA

VWR International GmbH

Germany

Labortect

Germany

JASCO Deutschland GmbH

Germany

Binder GmbH

Germany

Bio-Rad

USA

Elementar Analysensysteme

Germany $\mathrm{GmbH}$

Bio-Rad

USA

Martin Christ

Germany

Gefriertrocknungsanlagen $\mathrm{GmbH}$

Perkin Elmer

USA 
Name of the device

- $\quad$ GEL iX20 Imager (windows version)

- $\quad$ HPLC-GPC system is composed of:

i. $\quad$ pump (L 7100),

ii. auto sampler (AS-2000 A),

iii. GPC_MCX column $(8 \times 30 \mathrm{~mm})$,

iv. $\quad$ Detector (Shodex ${ }^{\circledR}$ RI-10), and

v. Clarity GPC Extension software for data analysis

- HPLC-UV-ESI-MS system (Ultimate 3000RS) is composed of:

i. degasser (SRD 3400),

ii. a pump module (HPG 3400RS),

iii. an autosampler (WPS 3000TRS),

iv. column compartment (TCC 3000RS),

v. column (Gravity C18, 100x2 $\mathrm{mm}, 1.8 \mu \mathrm{m}$ particle size; Macherey-Nagel)

vi. diode array detector (DAD 3000RS),

vii. an ESI-ion-trap unit (HCT; Bruker),

viii. Accurate-Post-Column-Splitter, and

ix. Bruker Hystar, QuantAnalysis and Dionex Chromelion software for data collection and analysis.

\section{Company}

Intas Science Imaging Instruments

$\mathrm{GmbH}$

- Merck-Hitatchi

- PSS

- Shimadzu Corporation

- Clarity

Dionex, Thermo Fisher Scientific

\section{Country}

Germany

- Germany

- Japan

- Czech Republic

Germany 
Name of the device

- Magnetic stirrer with heating (MR Hei-Standard)

- Melting point apparatus (DigiMelt-MPA 160)

- Microfluidizer (Microfluidics)

- $\quad$ Microplate reader (EL808) provided with Microplate Data Collection \& Analysis Software

- $\quad$ Microscope (ECLIPSE Ni-U) with NIS-Element software

- Nanodrop spectrophottometer, with NanoDrop 2000/2000c Software

- $\quad$ NMR Spectrometer (400 MHz, Brucker ${ }^{\circledR} 600$ Ultrashield)

- Orbital shaker (unimax 1010)

- Overhead shaker (Multi Bio RS24)

- $\quad$ PCR thermocycler (T-Gradient thermoblock)

- $\quad$ pH meter $(\mathrm{pH} 211)$

- Photoincubator (AlgaTron AG230 ECO)

- Rotary evaporator (Laborota 4003)

- Shaker incubator (Infors HT Ecotron)

- $\quad$ Shaker incubator (Innova ${ }^{\circledR} 44$ incubator shaker series)

- $\quad$ Spectrofluorometer (FP-8300)

\section{Company}

Country

Heidolph Instruments GmbH \& Co.

Germany

KG

SRS Scientific Instruments GmbH

Germany

Quadro Engineering Corp.,

Canada

BioTek Instrument

USA

Nikon

Japan

Thermo Scientific

USA

Brucker

Germany

Heidolph

Germany

Biosan

Latvia

Analytik Jena AG

Germany

Hanna ${ }^{\circledR}$ Instrument

USA

PSI

Czech Republic

Heidolph Instruments GmbH \&

Germany

$$
\text { Co.KG }
$$

Infors AG

Switzerland

New Brunswick

Germany

Scientific/Eppendorf AG

JASCO Deutschland GmbH
Germany 
Name of the device

- Superspeed Centrifuge (SORVALL LYNX $^{\circledR}$ 6000)

- $\quad$ Thermoshaker (TS-100)

- $\quad$ Tissuelyzer (MM 200)

- $\quad$ Top shaker (Rocker 3D basic)

- Ultrasonic bath (Sonorex ${ }^{\circledR}$ Digital 10 P)

- UV/Vis-spectrometer (Cary 60 Uv-Vis)

- Vertical electrophoresis cell for SDS-PAGE (Mini-PROTEAN ${ }^{\circledR}$

Tetra cell) with PowerPac ${ }^{\mathrm{TM}}$

Basic Power Supply

- Visible spectrophotometer (Amersham Biosciences

Einstrahl-Spektralfotometer Novaspec III)

- Water ultra-purification system (Ultra Clear TWF/ El-ION UV plus $^{\mathrm{TM}}$ )

- Wave bag bioreactor (BIOSTAT $\left.{ }^{\circledR} \mathrm{RM}\right)$

\section{Company}

ThermoFischer Scientific

Country

USA

Biosan

Latvia

Retsch

Germany

IKA®-Werke GmbH \& Co. KG

Germany

Bandelin

Germany

Agilent Technologies

USA

Bio-Rad

USA

Klüver \& Schulz GmbH

Germany

Siemens/Evoqua water technologies

Germany

Sartorious Stedim Biotech GmbH

Germany 


\section{Publications in peer-reviewed journals and conferences}

Partial results from this work have been pre-published in the following papers.

Journals:

1. A. Zayed, C. Dienemann, C. Giese, R. Krämer, R. Ulber, "An immobilized perylene diimide derivative for fucoidan purification from a crude brown algae extract," Process Biochem., 2018. 65, 233 - 238. DOI: 10.1016/j.procbio.2017.10.012

2. A. Zayed, R. Ulber, "Heterologous Expression of Heteropolysaccharides," Chem. Ing. Tech. 2016. 88(9), 1399. DOI: $10.1002 /$ cite.201650230

3. A. Zayed, R. Ulber, "Downstream Processing of Bioactive Heteropolysaccharides," Chem. Ing. Tech. 2016. 88(9), 1398. DOI: $10.1002 /$ cite. 201650238

4. A. Zayed, K. Muffler, T. Hahn, S. Rupp, D. Finkelmeier, A. Burger-Kentischer, and R. Ulber, "Physicochemical and Biological Characterization of Fucoidan from Fucus vesiculosus Purified by Dye Affinity Chromatography," Mar. Drugs 2016. 14(4), 79. DOI: 10.3390/md14040079.

5. T. Hahn, M. Schulz, R. Stadtmüller, A. Zayed, K. Muffler, S. Lang, and R. Ulber, "A Cationic Dye for the Specific Determination of Sulfated Polysaccharides". Anal. Lett., 2016. 2719, 1948-1962. DOI: 10.1080/00032719.2015.1126839.

6. T. Hahn, A. Zayed, M. Kovacheva, R. Stadtmüller, S. Lang, K. Muffler, and R. Ulber, "Dye affinity chromatography for fast and simple purification of fucoidan from marine brown algae," Eng. Life Sci., 2016. 16(1), 78-87. DOI: 10.1002/elsc.201500044.

\section{Talk in Conferences:}

1. A. Zayed, T. Hahn, S. Rupp, R. Krämer, R. Ulber, "Fucoidan as a Natural Anticoagulant, Antiviral and Anti-cancer Drug", 3rd German Pharm-Tox Summit 2018 (26 February- 1 March 2018, Göttingen, Germany).

2. A. Zayed, R. Ulber. "Downstream processing of bioactive heteropolysaccharides". ProcessNetJahrestagung und 32. DECHEMA-Jahrestagung der Biotechnologen 2016. (12.- 15. September 2016, Aachen, Germany).

3. A. Zayed, K. Muffler, R. Ulber. "Novel and rapid extraction and purification procedure for fucoidan from F. vesiculosus". Jahrestreffen der Fachgruppe Phytoextrakte $\left(23^{\text {rd }}\right.$ February 2015, Bonn, Germany).

\section{Poster:}

1. A. Zayed, C. Dienemann, C. Giese, R. Krämer, R. Ulber. "Fucoidan Purification: A Scale-Up Approach". Himmelfahrtstagung 2018: Heterogeneities - A key for understanding and upscaling of bioprocesses in up- and downstream ( $7-9^{\text {th }}$ May 2018 Herrenkrug Parkhotel, Magdeburg, Germany).

2. A. Zayed, C. Dienemann, R. Krämer, R. Ulber. "A Perylene Diimide Derivative for Sulphated Heteropolysaccharides Purification (e.g., Fucoidan from Crude Brown Algae Extract)". French Chemical and Process Engineering Society (SFGP), (July 11-13 $3^{\text {th }} \mathbf{2 0 1 7}$, Nancy, France - GermanFrench day - July $12^{\text {th }}, 2017$ ).

3. A. Zayed, C. Dienemann, R. Krämer, R. Ulber. "A simple and Time-saving Purification of Fucoidan from Crude Brown Algae Extracts. Himmelfahrtstagung 2017: Models for Developing and Optimising Biotech Production, (May 22. - 24. 2017, Neu-Ulm, Germany).

4. A. Zayed, B. Ledermann, N. Frankenberg-Dinkel, R. Ulber. "Evaluation of Heterologously Expressed Algal Fucosyltransferases and Sulfotransferases in E. coli'. Himmelfahrtstagung 2017: Models for Developing and Optimising Biotech Production, (22. - 24. May 2017, Neu-Ulm, Germany). 
5. A. Zayed, B. Ledermann, N. Frankenberg-Dinkel, R. Ulber. "Heterologous expression of heteropolysaccharides". ProcessNet-Jahrestagung und 32. DECHEMA-Jahrestagung der Biotechnologen 2016, (12. - 15. September 2016, Aachen, Germany).

6. A. Zayed, K. Muffler, T. Hahn, R. Ulber. "Cultivation of macro algae in wave bag reactors for the production of sulfated heteropolysaccharides". Himmelfahrtstagung 2016: New Frontiers for Biotech-Processes (2. - 4. May 2016, Koblenz, Germany 



\section{Curriculum Vitae}

\section{Personal information}

Name:

Birth place:

\section{Education}

$$
1987-1991
$$

$1992-1995$

$1996-1998$

$1998-2003$

$2003-2012$

$2004-2006$

$2006-2012$
Ahmed Elsayed Ahmed Farag Zayed

Tanta/Gharbeya, Egypt
Ground school in Tanta/Gharbeya, Egypt

Preparatory school Mehalla Elkobra/Gharbeya, Egypt

Secondary school in Mehalla Elkobra/Gharbeya, Egypt

Faculty of Pharmacy, Tanta University, Egypt

Final degree: B. Sc. in Pharmaceutical sciences (Excellent with honor,

Rank: $\left.4^{\text {th }}\right)$

Instructor

Pharmacognosy Department,

Faculty of Pharmacy, Tanta University, Egypt

Pre-master student

Pharmacognosy Department,

Faculty of Pharmacy,

Tanta University, Egypt

M. Sc. Student

Pharmacognosy Department,

Faculty of Pharmacy,

Tanta University, Egypt

Final Degree: Passed. M. Sc. in Pharmaceutical sciences (Pharmacognosy) 
Since 2012

$4 / 2014-3 / 2018$

Since 4/2018
Assistant lecturer

Pharmacognosy Department,

Faculty of Pharmacy,

Tanta University, Egypt

Doctoral student as a DAAD scholarship holder (GERLS program)

Bioprocess Engineering Institute,

Prof. Dr. rer. nat. Roland Ulber

TU Kaiserslautern, Germany

Doctoral student as an insititional scholarship holder

Bioprocess Engineering Institute,

Prof. Dr. rer. nat. Roland Ulber

TU Kaiserslautern, Germany 